\title{
Evidence-based guidelines for the pharmacological treatment of schizophrenia: updated recommendations from the British Association for Psychopharmacology
} DOI:

$10.1177 / 0269881119889296$

\section{Document Version}

Final published version

Link to publication record in Manchester Research Explorer

Citation for published version (APA):

Barnes, T. R. E., Drake, R., Paton, C., Cooper, S. J., Deakin, J. F. W., Ferrier, I. N., Gregory, C., Haddad, P., Howes, O., Jones, I., Joyce, E. M., Lewis, S., Lingford-Hughes, A., MacCabe, J., Owens, D. C., Patel, M., Sinclair, J., Stone, J., Talbot, P., ... Yung, A. (2020). Evidence-based guidelines for the pharmacological treatment of schizophrenia: updated recommendations from the British Association for Psychopharmacology. Journal of psychopharmacology (Oxford, England), 34(1), 3-78. https://doi.org/10.1177/0269881119889296

Published in:

Journal of psychopharmacology (Oxford, England)

\section{Citing this paper}

Please note that where the full-text provided on Manchester Research Explorer is the Author Accepted Manuscript or Proof version this may differ from the final Published version. If citing, it is advised that you check and use the publisher's definitive version.

\section{General rights}

Copyright and moral rights for the publications made accessible in the Research Explorer are retained by the authors and/or other copyright owners and it is a condition of accessing publications that users recognise and abide by the legal requirements associated with these rights.

Takedown policy

If you believe that this document breaches copyright please refer to the University of Manchester's Takedown Procedures [http://man.ac.uk/04Y6Bo] or contact uml.scholarlycommunications@manchester.ac.uk providing relevant details, so we can investigate your claim.

\section{OPEN ACCESS}




\section{Evidence-based guidelines for the pharmacological treatment of schizophrenia: Updated recommendations from the British Association for Psychopharmacology}

\author{
Thomas RE Barnes ${ }^{1}$ iD, Richard Drake ${ }^{2}$, Carol Paton $^{3}$ iD, Stephen J Cooper ${ }^{4}$, Bill Deakin $^{5}$, \\ I Nicol Ferrier ${ }^{6}$, Catherine J Gregory ${ }^{7}$, Peter M Haddad ${ }^{8}$, Oliver D Howes ${ }^{9}$, Ian Jones ${ }^{10}$, \\ Eileen M Joyce ${ }^{11}$, Shôn Lewis ${ }^{12}$, Anne Lingford-Hughes ${ }^{13}$, James H MacCabe ${ }^{14}$, \\ David Cunningham Owens ${ }^{15}$, Maxine $X$ Patel $^{16}$, Julia MA Sinclair ${ }^{17}$, James M Stone ${ }^{18}$ iD, \\ Peter S Talbot ${ }^{19}$, Rachel Upthegrove ${ }^{20}$, Angelika Wieck ${ }^{21}$ and Alison R Yung ${ }^{22}$
}

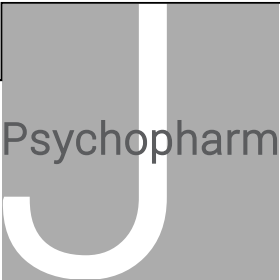

Journal of Psychopharmacology 2020, Vol. 34(1) 3-78 (c) The Author(s) 2019 Article reuse guidelines: sagepub.com/journals-permissions DOI: $10.1177 / 0269881119889296$ journals.sagepub.com/home/jop

(SAGE

\begin{abstract}
These updated guidelines from the British Association for Psychopharmacology replace the original version published in 2011. They address the scope and targets of pharmacological treatment for schizophrenia. A consensus meeting was held in 2017, involving experts in schizophrenia and its treatment. They were asked to review key areas and consider the strength of the evidence on the risk-benefit balance of pharmacological interventions and the clinical implications, with an emphasis on meta-analyses, systematic reviews and randomised controlled trials where available, plus updates on current clinical practice. The guidelines cover the pharmacological management and treatment of schizophrenia across the various stages of the illness, including first-episode, relapse prevention, and illness that has proved refractory to standard treatment. It is hoped that the practice recommendations presented will support clinical decision making for practitioners, serve as a source of information for patients and carers, and inform quality improvement.
\end{abstract}

\title{
Keywords
}

Guideline, schizophrenia, psychopharmacology

\footnotetext{
${ }^{1}$ Emeritus Professor of Clinical Psychiatry, Division of Psychiatry, Imperial College London, and Joint-head of the Prescribing Observatory for Mental Health, Centre for Quality Improvement, Royal College of Psychiatrists, London, UK

${ }^{2}$ Clinical Lead for Mental Health in Working Age Adults, Health Innovation Manchester, University of Manchester and Greater Manchester Mental Health NHS Foundation Trust, Manchester, UK 3Joint-head of the Prescribing Observatory for Mental Health, Centre for Quality Improvement, Royal College of Psychiatrists, London, UK 4Emeritus Professor of Psychiatry, School of Medicine, Queen's University Belfast, Belfast, UK

${ }^{5}$ Professor of Psychiatry, Neuroscience \& Psychiatry Unit, University of Manchester and Greater Manchester Mental Health NHS Foundation Trust, Manchester, UK

${ }^{6}$ Emeritus Professor of Psychiatry, Institute of Neuroscience, Newcastle University, Newcastle upon Tyne, UK

${ }^{7}$ Honorary Clinical Research Fellow, University of Manchester and Higher Trainee in Child and Adolescent Psychiatry, Manchester University NHS Foundation Trust, Manchester, UK ${ }^{8}$ Honorary Professor of Psychiatry, Division of Psychology and Mental Health, University of Manchester, UK and Senior Consultant Psychiatrist, Department of Psychiatry, Hamad Medical Corporation, Doha, Qatar ${ }^{9}$ Professor of Molecular Psychiatry, Imperial College London and Institute of Psychiatry, Psychology and Neuroscience, King's College London, London, UK

${ }^{10}$ Professor of Psychiatry and Director, National Centre of Mental Health, Cardiff University, Cardiff, UK

${ }^{11}$ Professor of Neuropsychiatry, UCL Queen Square Institute of Neurology, London, UK

${ }^{12}$ Professor of Adult Psychiatry, Faculty of Biology, Medicine and Health, The University of Manchester, UK, and Mental Health Academic Lead, Health Innovation Manchester, Manchester, UK ${ }^{13}$ Professor of Addiction Biology and Honorary Consultant Psychiatrist, Imperial College London and Central North West London NHS Foundation Trust, London, UK
}

14Professor of Epidemiology and Therapeutics, Department of Psychosis Studies, Institute of Psychiatry, Psychology and Neuroscience, King's College London, and Honorary Consultant Psychiatrist, National Psychosis Service, South London and Maudsley NHS Foundation Trust, Beckenham, UK

${ }^{15}$ Professor of Clinical Psychiatry, University of Edinburgh. Honorary Consultant Psychiatrist, Royal Edinburgh Hospital, Edinburgh, UK ${ }^{16}$ Honorary Clinical Senior Lecturer, King's College London, Institute of Psychiatry, Psychology and Neuroscience and Consultant Psychiatrist, 0xleas NHS Foundation Trust, London, UK

${ }^{17}$ Professor of Addiction Psychiatry, Faculty of Medicine, University of Southampton, Southampton, UK

${ }^{18}$ Clinical Senior Lecturer and Honorary Consultant Psychiatrist, King's College London, Institute of Psychiatry, Psychology and Neuroscience and South London and Maudsley NHS Trust, London, UK

${ }^{19}$ Senior Lecturer and Honorary Consultant Psychiatrist, University of Manchester and Greater Manchester Mental Health NHS Foundation Trust, Manchester, UK

20Professor of Psychiatry and Youth Mental Health, University of Birmingham and Consultant Psychiatrist, Birmingham Early Intervention Service, Birmingham Women's and Children's NHS Foundation Trust, Birmingham, UK

${ }^{21}$ Honorary Consultant in Perinatal Psychiatry, Greater Manchester Mental Health NHS Foundation Trust, Manchester, UK

22Professor of Psychiatry, University of Manchester, School of Health Sciences, Manchester, UK and Centre for Youth Mental Health, University of Melbourne, Australia, and Honorary Consultant Psychiatrist, Greater Manchester Mental Health NHS Foundation Trust, Manchester, UK

\section{Corresponding author:}

Thomas R E Barnes, Emeritus Professor of Clinical Psychiatry, Division of Psychiatry, Imperial College London, Du Cane Road, London, W12 ONN, UK.

Email: t.r.barnes@imperial.ac.uk 


\section{Introduction}

The methodology for this updated guideline was essentially the same as was followed for previous British Association for Psychopharmacology (BAP) guidelines. A meeting of experts was convened in May 2017 with a remit to produce up-to-date, evidence-based, consensus recommendations covering the pharmacological management of schizophrenia from first episode to long-term relapse prevention, targeting treatment for particular persistent symptoms, and strategies for refractory illness. All the contributors invited to the meeting had extensive clinical experience of the treatment of people with schizophrenia and each of them gave a brief presentation of the published evidence relevant to the section of the guideline they would write. The costs of the meeting were defrayed by the BAP. Subsequently, the submissions from each of the co-authors were edited and integrated into a first draft which was then circulated for further amendment and updating in 2018. A second draft was similarly revised and updated in 2019.

\section{Strength of evidence and recommendations for guidelines}

To rank the evidence and the strength of recommendations, the authors followed the categories used in the earlier, published version of this guideline (Barnes et al., 2011), which had been taken from the methodology of the North of England Evidence-Based Guideline Development Project, developed by the Centre for Health Services Research, University of Newcastle upon Tyne and the Centre for Health Economics, University of York.

Evidence categories. The six evidence categories (see Shekelle et al., 1999) are as follows:

\section{Categories of evidence for causal relationships and treatment}

Ia: evidence from meta-analysis of randomised controlled trials (RCTs)

$\mathrm{Ib}$ : evidence from at least one RCT

IIa: evidence from at least one controlled study without randomisation

IIb: evidence from at least one other type of quasi-experimental study

III: evidence from non-experimental descriptive studies, such as comparative studies, correlation studies and case-control studies

IV: evidence from expert committee reports or opinions and/ or clinical experience of respected authorities

This categorisation is most appropriate for questions of short-term efficacy. Similar taxonomies for other types of research question, including long-term impacts and real-world effectiveness, do not yet exist (Davis et al., 2019) and the following is proposed.

\section{Proposed categories of evidence for non-causal relationships}

I: evidence from large representative population samples

IIa: evidence from small, well-designed, but not necessarily representative samples
IIb: evidence from pharmacovigilance studies

III: evidence from non-representative surveys, case reports

IV: evidence from expert committee reports or opinions and/ or clinical experience of respected authorities

\section{Strength of recommendation}

A: directly based on category I evidence

B: directly based on category II evidence or extrapolated recommendation from category I evidence

C: directly based on category III evidence or extrapolated recommendation from category I or II evidence

D: directly based on category IV evidence or extrapolated recommendation from category I, II or III evidence

The recommendations are graded A to D, as shown above. We distinguish between the category of evidence and the strength of the associated recommendation. It is possible to have methodologically sound (category I) evidence about an area of practice that is clinically irrelevant or where the effect is modest and of little practical significance and therefore a lower strength of recommendation is warranted. More commonly, a statement of evidence covers only one aspect of a recommendation or covers it in a way that conflicts with other evidence. Therefore, to produce comprehensive recommendations it is necessary to generalise from the available evidence. This may lead to weaker levels of recommendation (B, C or D) based upon category I evidence statements.

Deriving realistic, sound recommendations for clinical practice from a disparate and inconsistent evidence base is not straightforward; it is not simply the application of deductive reasoning or simple extrapolation. Critical appraisal of research studies and judicious reflection on clinical experience are necessary, as well as some insight into the cognitive biases and defaults involved in the decision-making process. Where recommendations were predominantly derived from a consensus view, in the absence of valid, systematic evidence, they are graded as S (standard of good practice).

\section{Nomenclature}

Antipsychotic medication is mentioned frequently throughout the text and should be taken to refer to medications in the 'drugs for psychosis' section of the Neuroscience based Nomenclature (Nutt and Blier, 2016), which have a range of putative modes of action, including the following: receptor antagonist (D2); receptor antagonist (D2, 5-HT2); receptor partial agonist (D2, 5-HT1A); and receptor antagonist (D2, 5-HT2, NE alpha-2).

\section{The at-risk mental state}

Early intervention in the first episode of psychosis has the potential to improve outcomes. In the light of this, in the late 1990s consideration was given to whether even earlier intervention was possible, specifically during the prodromal phase preceding the first psychotic episode. The hypothesis to be tested was that such intervention could result in attenuation, delay or even prevention of the onset of psychosis in some individuals (McGlashan et al., 2003; Yung et al., 1996). Criteria were developed to allow the identification of young people at high and imminent risk of developing a first episode of psychosis (McGlashan et al., 2003; Yung et al., 1996). 
These have been referred to as the prodromal, ultra-high risk (UHR), clinical high-risk (CHR) and at-risk mental state (ARMS) criteria (Fusar-Poli et al., 2013a). The accepted ARMS criteria have now been in use worldwide for over 20 years. They have shown predictive validity for psychotic disorders across different countries and service settings. While the risk of individuals fulfilling ARMS criteria developing a full psychotic disorder has been calculated to be $15-30 \%$ within 12 months, and over $36 \%$ after 3 years (FusarPoli et al., 2012), lower rates of transition have been reported, such as $8 \%$ of 288 ARMS subjects at 1-2 years (Morrison et al., 2012).

The potential objectives of pharmacotherapy in ARMS cases that come under the care of clinical services are threefold: reduction in attenuated psychotic symptoms; prevention, delay or amelioration of the onset of psychosis; and rapid detection and early intervention in the first episode of psychosis.

\section{Interventions to reduce attenuated psychotic symptoms}

For those individuals who are seeking help for their presenting attenuated psychotic symptoms, very low-dose antipsychotic medication can be considered for short-term symptom relief, although such a prescription would be 'off-label' in terms of indication, and there are only limited trial data to inform dosage. In general, the dosages of antipsychotic medication prescribed for people with an ARMS are even lower than those used in firstepisode psychosis, as affected individuals tend to be exquisitely sensitive to both the therapeutic effects and adverse effects of such medication (McGlashan et al., 2006; McGorry et al., 2002; Ruhrmann, 2007). However, individuals with an ARMS are often reluctant to take medication, and frequently express a preference for psychological intervention (Broome et al., 2005).

There is preliminary evidence that low-dose antipsychotic medication, cognitive behavioural therapy (CBT) and supportive therapy can improve attenuated psychotic symptoms (Ruhrmann, 2007; Woods et al., 2003, 2007). Two trials that examined the combined effect of an antipsychotic medication plus CBT found no advantage for the combination above CBT alone (McGorry et al., 2002, 2013; Yung et al., 2011). The effect of antipsychotic medication without CBT was not assessed in these trials, but a randomised trial in treatment-seeking individuals showed a significant reduction in attenuated psychotic symptoms at 6-month follow-up in the participants receiving ziprasidone compared with those assigned to placebo (Woods et al., 2017). A pair-wise meta-analysis of six studies found evidence that CBT significantly improved attenuated psychotic symptoms compared with needs-based intervention alone (Davies et al., 2018b), and there is preliminary evidence that family therapy may also improve attenuated psychotic symptoms (Miklowitz et al., 2014). As all recent studies in this area examined the effect of one specific intervention compared with placebo and/ or supportive 'needs-based' treatment, the potential for psychopharmacological interventions to facilitate the therapeutic effects of psychological treatments remains largely unexplored.

\section{Interventions to reduce risk, delay or ameliorate the onset of psychotic disorder}

The second potential clinical objective is to delay, prevent or reduce the severity of the onset of a psychotic illness. The findings of a few relevant trials (McGlashan et al., 2006; McGorry et al., 2002; Morrison et al., 2004) suggest that this may be possible with either low-dose antipsychotic medication or CBT. There is meta-analytical evidence that both these treatments can reduce the risk of developing psychosis (van der Gaag et al., 2013). The number needed to treat (NNT) for antipsychotic treatment was 7 compared with 13 for CBT, suggesting that risk reduction is greater for the former (van der Gaag et al., 2013). There are two further antipsychotic trials that have examined efficacy to reduce the risk of transition to psychosis. A multi-site RCT comparing ziprasidone with placebo showed no significant effect, but the study was underpowered: recruitment targets were not met and transition rates were low (Woods et al., 2017). A three-armed trial comparing CBT, aripiprazole plus case management, and placebo plus case management found no significant differences between the treatment groups (Bechdolf et al., 2016).

One issue is that the studies of antipsychotic medication tend to show high attrition rates (McGlashan et al., 2006; Woods et al., 2017), suggesting that this treatment may be poorly accepted and/or poorly tolerated in the ARMS group. Further studies are needed to assess the efficacy and safety of these medications. Ongoing multicentre trials with much larger patient samples would be necessary to provide definitive findings.

A single-site, randomised, placebo-controlled trial of omega-3 polyunsaturated fatty acids (PUFA) showed a significant reduction in transition rate in the intervention group (Amminger et al., 2010). However, this positive finding was not replicated in two larger multi-site studies (Cadenhead et al., 2017; McGorry et al., 2017).

Data are also available on the efficacy of antidepressants in ARMS patients, although to date these have been derived from clinical audits rather than clinical trials. Cornblatt et al. (2007) reported a very low risk of transition to psychosis in individuals with high-risk features who had been treated with antidepressants, as opposed to antipsychotic medication. Similar findings emerged from an audit of treatment of at-risk subjects in the UK (Fusar-Poli et al., 2007). But it is unclear to what extent these low rates of transition in observational studies are attributable to a therapeutic effect of the drug treatment or explicable by factors such as inadequate duration of follow-up or indication bias.

A network meta-analysis found that no specific pharmacological treatment was superior to 'needs-based intervention' for reducing transition risk over 6 and 12 months (Davies et al., 2018a). Longer follow-up periods were not examined due to a lack of data. However, this network meta-analysis combined data on needs-based intervention from multiple studies, despite the likelihood that the nature of such intervention would differ markedly across studies, not least in the quality of the relationship between patients and the relevant mental health professionals assessing need and delivering any intervention.

\section{Rapid detection and early intervention in the first episode of psychosis}

The third objective for treatment is to intervene as soon as psychosis develops, in order to improve the subsequent outcome. If subjects at high risk have already been engaged by mental health services before the onset of illness, the delay between the onset of frank psychosis and the initiation of antipsychotic treatment can be substantially reduced. For example, in South London the 
mean duration of untreated psychosis (DUP) in patients who developed psychosis after presenting to a service for people with an ARMS was 10 days, as compared with 12 months in patients whose first contact with mental health services was after the onset of illness (Valmaggia et al., 2009). Those patients who received treatment at the ARMS service had a better short-term clinical outcome than those patients who did not present until the first episode (Valmaggia et al., 2015). However, the better clinical outcome in this group may be related, at least in part, to the demographic profile of the selected group who accessed the ARMS service (Fusar-Poli et al., 2013b; Morgan et al., 2017).

\section{Substance use in ARMS patients}

The literature is mixed regarding the extent of substance use in ARMS populations, and may depend on the service in which the research was conducted. Substance use is rarely the outcome of interest in this group and some ARMS studies exclude people with substance use. While some researchers have found low rates of substance use in ARMS services (Fusar-Poli et al., 2013b; Lin et al., 2015), one systematic review and meta-analysis showed that ARMS patients smoke more cigarettes than controls (Carney et al., 2016) while another found that ARMS patients have high rates of cannabis use $(27 \%$ current users, $53 \%$ lifetime users) (Carney et al., 2017). There have been no studies investigating specific interventions for substance use in ARMS individuals.

\section{Comorbid psychiatric conditions}

Finally, an under-researched area is the potential to treat comorbid psychiatric symptoms and syndromes, such as depression and anxiety. These symptoms, even to a level of diagnosable disorder, are common in people fulfilling ARMS criteria: around $40 \%$ have a depressive disorder at baseline and about $30 \%$ have an anxiety disorder (Fusar-Poli et al., 2014; Lin et al., 2015). Antidepressant and antipsychotic medications may also play a role in the treatment of these symptoms in ARMS patients. In clinical trials, antipsychotic treatment has been associated with an improvement in both depressive and anxiety symptoms in this group (Ruhrmann, 2007; Woods et al., 2003, 2007). However, these comorbid syndromes have not been the primary target in any recent trials of either psychological or psychopharmacological treatment.

\section{Recommendations for the at-risk mental state}

- Encourage a therapeutic relationship to allow for further assessment, review, 'watchful waiting' and monitoring of symptoms. (S)

- Assess the nature and impact of any substance use (see the section below on Pharmacological strategies for comorbid substance use). (S)

- If antipsychotic medication is considered for symptom relief:

- This should be treated as off-label prescribing. (S)

- The prescription should be treated as a short-term, individual trial. (D)
○ Very low doses should be used. (D)

- Symptom response should be monitored. (D)

- The nature and frequency of potential side effects should be explained before a patient starts medication and then side effects should be carefully monitored during treatment. (D)

- It should be prescribed by specialist psychiatric services, such as an early intervention team. (D)

- On the evidence available, individual CBT can be considered to be an acceptable alternative to pharmacological treatment. (D)

\section{Key uncertainties}

Areas of clinical uncertainty are the value of antipsychotic medication in preventing or delaying the onset of psychotic illness, the value of antidepressants in reducing the risk of developing psychosis, the value of any novel agents, such as omega-3 PUFA, to relieve symptoms, and the appropriate dose and duration of these pharmacological treatments. Any evidence of benefit from a pharmacological intervention would need to be weighed against the harms of possible side effects and the potential risk of stigma and self-stigmatisation in ARMS individuals prescribed medication (Corcoran et al., 2005). However, it may be that the symptoms themselves and fear of becoming psychotic are more stigmatising for some patients than receiving an intervention (Corcoran, 2016; Yang et al., 2015).

Although relevant pharmacological trials are ongoing, one key issue is that the rate of transition to psychotic disorder within the ARMS group appears to be declining (Hartmann et al., 2016; Yung et al., 2007). Thus, strategies to enrich study samples for increased risk of psychosis may be warranted. Further, other treatments targets should be considered for pharmacological trials, such as reduction in attenuated psychotic symptoms, social disability and comorbid psychopathology.

\section{First-episode psychosis}

\section{Diagnosis and assessment}

A diagnosis of a psychotic disorder can often be made early in the course of illness, but for some patients the clinical presentation evolves over time, with symptoms changing and emerging, and only later does the diagnosis become clear (longitudinal observational analyses: Schimmelmann et al., 2005; Schwartz et al., 2000; Tarrier et al., 2004). Moreover, there are meaningful differences in presentation, prognosis and management between acute and transient psychosis (ICD-10 category F23); substanceinduced psychotic disorders (F1x.0, F1x.4-5; Caton et al., 2007); non-affective psychoses like schizophrenia (F20, F22, F25); and affective psychoses (F30-33; Kotov et al., 2013; Morgan et al., 2014), even if combining categorical and dimensional descriptions increases the prognostic power (Demjaha et al., 2009). Failure to make a diagnosis can deny patients timely access to treatments that are most appropriate for particular disorders. It can also lead to the inappropriate discharge from hospital of those people with persistent illness or to the inappropriate continuation of antipsychotic medication for those who would be 
very unlikely to relapse after acute episodes. There is evidence that failure to diagnose psychosis can lead to long delays in treatment (Marshall et al., 2014). Thus, in most cases it may be better to make an initial diagnosis but be prepared to revise it should the clinical picture alter or become clarified.

The preferred management and assessment strategies for transient psychotic disorders (F23) and substance-induced psychotic disorders (F1x.5) are expectant management without antipsychotic medication for up to 2 weeks before reviewing diagnosis; or initiating and then discontinuing such medication in the event of a rapid response. The clinical response to antipsychotic treatment can make the distinction between these disorders and schizophrenia or other non-affective psychoses less clear. Provided there is little risk of self-harm or harm to others, and substance use has ceased, expectant management may be preferable. But the more vulnerable a person might be, the higher their risk of self-harm or violence despite attempts to mitigate risk; the more likely they are to have schizophrenia, the more the early use and evaluation of antipsychotic medication should be considered. Factors that suggest a diagnosis of schizophrenia rather than an alternative include a long history of psychosis, a family history of schizophrenia, and relatively intermittent substance use and poor insight (less typical for F1x.5; Caton et al., 2007). GABA A $_{\text {receptor modulators, such as }}$ benzodiazepines for anxiety and insomnia or other sedatives such as zopiclone for insomnia, might be appropriate for short periods (e.g. 2 weeks) as part of expectant management. Benzodiazepine drugs commonly used for agitation and anxiety in inpatients include diazepam, lorazepam, or clonazepam (though benzodiazepine medications with a long half-life, such as diazepam and clonazepam, will accumulate with regular use and are therefore easily overdosed, thus warranting particular care and caution if prescribed for community patients). Typical medications for insomnia include nitrazepam, zopiclone or zolpidem.

Post-traumatic stress disorder (F31.1) or other complications of childhood and/or adult trauma are differential diagnoses but also commonly comorbid. They represent targets for psychological treatment, and may affect presentation, outcome, and relationships with services. Comorbid use or dependence on alcohol or other drugs may be concealed.

Beyond routine history, mental state and physical examination (with measurement of body weight and calculation of body mass index: BMI), the personal history of illness should include any concomitant physical disorders or relevant past disorders, in particular cardiac and neurological disease, gestational diabetes and febrile seizures. Family history should include physical disorders that might increase the risk of harm from antipsychotic medication, such as a myocardial infarct or sudden cardiac death in a first-degree relative before the age of 60 years (HippisleyCox et al., 2008), type 2 diabetes, or epilepsy.

It is also essential to exclude intoxication (F1x.0), withdrawal (F1x.4) or substance-induced psychotic disorders (F1x.5), organic delusional disorders (F06), delirium (F05) and dementia (F00-03). All patients should have a full physical examination (including fundoscopy). Depending on the clinical picture and circumstances, screening for causes of organic delusional disorder, substance use or physical disorders that could complicate treatment should include some of the following investigations:

- Electrolytes and urea and estimated glomerular filtration rate (eGFR), plasma glucose, liver function tests, thyroid function tests, plasma lipid profile, c-reactive protein (CRP) or plasma viscosity (PV) or erythrocyte sedimentation rate (ESR), and blood calcium level.

- Urine drug screen.

- Electrocardiogram (ECG). If possible, services should perform a baseline ECG to exclude abnormalities (Howell et al., 2019) before antipsychotic medication or any potential cardiotoxic medications are initiated (see also section below, Mortality cardiovascular disease and antipsychotic treatment). The patient's family history (up to third-degree relatives) should be checked for sudden cardiac death and account taken of the presence of physical illness such as liver or cardiac disease, or concomitant medications that interact pharmacokinetically or pharmacodynamically with antipsychotic medication (Girardin et al., 2013). An ECG should ideally be repeated after a week if any abnormalities were identified in the baseline ECG or an antipsychotic medicine associated with a higher risk of cardiac conduction problems is being prescribed. The risk of sudden cardiac death appears highest in first two weeks of antipsychotic treatment, declining by 3 months to a level of risk possibly no different from the general population (largescale, within-individual case-control studies: Wu et al., 2015; van Noord et al., 2011). Any difficulty encountered in obtaining an ECG should increase efforts to screen for additional risk factors for arrhythmia, including low serum magnesium (especially if serum potassium is low); and consideration of prescribing antipsychotic medication less likely to cause lengthening of the QTc (see meta-analytic evidence from Leucht et al., 2013 and Huhn et al., 2019). Incidentally, the heightened relative risk of death may be due to the risk of acute myocardial infarction (within-individual, casecontrol comparisons: Brauer et al., 2015), which like the risk of arrhythmia and risk of cerebrovascular accident (observational studies, e.g. Wu et al., 2015; or RCTs, e.g. de Deyn et al., 2005) is highest in the first week or two of treatment and little elevated, if at all, after 2-3 months. The relative risk of arrhythmia may reach around 2 with the start of antipsychotic monotherapy (e.g. Wu et al., 2015) and may be higher for polypharmacy (e.g. Girardin et al., 2013), but the absolute risk is very low for most people.

As a precursor to treatment with antipsychotic medication, the following investigations should also be conducted if practical: random (better still, fasting) plasma lipids, and glucose (or $\mathrm{HbA} 1 \mathrm{c})$ and prolactin.

Clinicians should also consider:

- Neuroimaging by computed tomography (CT) or structural magnetic resonance imaging (sMRI). Studies using volumetric abnormalities to stratify outcome in first-episode schizophrenia have not yet been replicated (Dazzan et al., 2015). Meta-analyses and large observational studies usually find space-occupying lesions are identified more frequently by imaging in this group, especially after sMRI (e.g. Albon et al., 2008; Falkenberget al., 2017; Lawrie et al., 1997), although not in one comparison of 
sMRI scans from patients with psychosis and healthy controls (Sommer et al., 2013). However, most lesions disclosed are non-progressive and neurodevelopmental in origin, such as arachnoid cysts; treatable space-occupying lesions are only rarely discovered by neuroimaging, arguably $0.5 \%$ of cases (Albon et al., 2008; Sommer et al., 2013), and thorough neurological examination is likely to reduce any added value of imaging even further. Nonetheless, when such lesions are identified, the consequences are life changing. Specific MRI sequences (e.g. FLARE) and specialist review may be appropriate when autoimmune disease is suspected.

- Electroencephalography (EEG) to exclude partial complex status epilepticus, delirium, and infectious or autoimmune encephalitis.

- Autoimmune screen, B12, folate.

- NMDAR, VGKR other receptor autoantibodies (the prevalence of these antibodies is potentially of the order of $1-5 \%$ but the actual prevalence of the antibodies or the disorders, and the effect of treatment, are not yet clear: Al-Diwani et al., 2019; Pollack et al., 2014; Rickards et al., 2014).

- Consider lumbar puncture (e.g. for infectious aetiology), metabolic disease screening, toxicology (e.g. heavy metal poisoning).

There is a stronger rationale for considering the latter group of investigations listed above for those patients without historical risk factors for a functional or toxic psychosis (such as a family history of psychosis or substance use) or whose illnesses either fail to respond (i.e. progress to Stage IIIa; Scott et al., 2013) or are very severe. Neurological abnormalities, especially altered consciousness and characteristic tics involving ipsilateral facial muscles and upper and lower limb flexion, can indicate limbic encephalitis or other receptor antibody encephalitides, as can incidental findings on physical investigation.

\section{Treatment delay and refusal}

In the period before treatment is initiated for schizophrenia, there is observational evidence of an elevated risk of harm to others $(40 \%$ of homicides by those diagnosed with schizophrenia occur before treatment; The National Confidential Inquiry into Suicide and Safety in Mental Health, 2018), and to self (meta-analysis indicates that suicide risk and serious self-harm risk may be greatly elevated relative to the general population; Dutta et al., 2010; Nielssen et al., 2012). In addition, delay in treatment can lead to deterioration of the illness, resulting in more severe symptoms in the medium term (Marshall et al., 2005). Across populations, such deterioration in treatment response appears to be most rapid in the early weeks of firstepisode psychosis, with less and less effect from progressively further delays until treatment (Boonstra et al., 2012; Drake et al., 2000; Harrigan et al., 2003). There are data suggesting that prolonged delays may be associated with a risk of violence (Winsper et al., 2013), and it is unclear whether suicide risk is greater; so although those patients presenting with long delays may not need urgent treatment, such decisions should only be made after careful risk assessment and followed by close monitoring. Given the suggestion of relatively high mortality in national case register studies (Tiihonen et al., 2009; Torniainen et al., 2015), and greater homicide risk in meta-analyses (Nielssen and Large, 2010) and national surveys (The National Confidential Inquiry into Suicide and Safety in Mental Health, 2018) in those people with schizophrenia who are not receiving antipsychotic treatment, discharging those with psychosis who come into contact with services but do not engage with any treatment appears likely to confer a relatively high risk of serious outcomes. For this reason alone, effective assessment of diagnosis and response to treatment is clearly mandated, if necessary by compulsory admission if the necessary legal requirements are met.

Intensive psychosocial interventions without the use of antipsychotic medication may be used for the treatment of psychosis (Bola and Mosher, 2003; Calton et al., 2008; May et al., 1981). There is evidence from a well-designed, randomised, controlled, rater-blind pilot trial that 6 months of CBT for psychosis (CBTp) in those declining to take indicated medication is efficacious against total symptoms (Morrison et al., 2014). In a further, raterblind feasibility trial, first-episode psychosis patients were randomised to CBTp or antipsychotic medication or both (Morrison et al., 2018); two-thirds of the participants were treated according to their allocated regimen. In this small study sample there was no significant difference between CBTp and antipsychotic medication in efficacy against symptoms over 1 year, although those participants allocated to the combined treatment benefited significantly more than those allocated to CBTp alone (with a trend towards greater efficacy than those allocated antipsychotic medication alone). Studies of family-based interventions without the prescription of antipsychotic medication have greater methodological limitations but suggested dramatic benefits (Bola et al., 2006; Falloon et al., 1985; Lehtinen et al., 2000). As yet almost all these approaches are unreplicated by other groups. It is not yet clear from rigorously designed, large, pragmatic RCTs whether interventions such as this are as effective as antipsychotic medication (Morrison et al., 2018), or even how efficacious they are outside specialist services with unique expertise. Further, typical CBTp for first-episode psychosis may have little effect on suicidality (Tarrier et al., 2006). Although rapid initiation of these treatments as well as antipsychotic medication is recommended by evidence-based guidelines such as NICE CG178 (NICE, 2014a) and QS14 (NICE, 2011a), the evidence for their recommendations comes almost entirely from trials combining psychological treatments with antipsychotic medication. The evidence that psychotherapy alone has effectiveness comparable to antipsychotic medication for first-episode psychosis is therefore very limited as yet, and necessarily depends on those cooperative and well enough to participate in trials. Clinicians considering compulsory treatment of patients potentially willing to engage in these psychotherapies but refusing indicated medication should bear all this in mind. Where the immediate risk of harm is low and patients can be engaged and monitored, and particularly if treatment delay has already been lengthy, it may be appropriate to evaluate progress with psychological therapy alone.

\section{Choice and dose of antipsychotic medication}

Low to moderate dosage of antipsychotic monotherapy is the first line of treatment for schizophrenia. The majority of first-episode illnesses will show a good response (Agid et al., 2013; Zhu et al., 2017). Doses should start well below those for established illness. 
Taking haloperidol as an example, an observational study of dopamine D2/3 receptor occupancy and efficacy of haloperidol for first-episode illness indicated that a median dose of haloperidol of $2 \mathrm{mg} /$ day was sufficient to achieve substantial D2/3 receptor occupancy, over $60 \%$ in most cases (Kapur et al., 2000). Moreover, the risk of adverse motor effects and prolactin elevation increased substantially with D2/3 receptor occupancies over about $80 \%$ (e.g. Kapur et al., 2000). This is in line with study findings regarding D2/3 receptor occupancy thresholds for establised illness (Uchida et al., 2011b). Similarly, the median threshold haloperidol doses for parkinsonian adverse effects are around $2 \mathrm{mg}$ of haloperidol in first-episode illness and $4 \mathrm{mg}$ in established illness (Miller et al., 2008; see also detailed discussion of this issue by Salimi et al., 2009). The efficacious mean olanzapine dose in firstepisode psychosis was identified by Stauffer et al. (2011) as 7.7 $\mathrm{mg}$. Large, pragmatic, variable-dose first-episode trials like the open European First-Episode Schizophrenia Trial (EUFEST) study (Kahn et al., 2008) provide a library of doses and adverse effects. Approximate, typical, daily mean effective doses for common antipsychotic medications are as follows: risperidone 2-4 $\mathrm{mg}$, haloperidol 2-5 mg, quetiapine 400-500 mg, amisulpride 450 $\mathrm{mg}$, and olanzapine $10 \mathrm{mg}$. Taken together, these data indicate that there is a therapeutic antipsychotic dose window associated with a high likelihood of response and low risk of motor and prolactin side effects, and that high doses generally increase the risk of side effects with limited additional therapeutic benefit in first-episode patients (Howes et al., 2009).

There is no clear indication from well-designed, double-blind RCTs or well-conducted meta-analyses (Zhang et al., 2013; Zhu et al., 2017) that any antipsychotic medication is more efficacious than any other for first-episode schizophrenia (e.g. McEvoy et al., 2007; Sikich et al., 2008). Olanzapine and amisulpride have outperformed haloperidol only in pharmaceutical industry-funded studies in one meta-analysis (Zhang et al., 2013). Risperidone, olanzapine and amisulpride also outperformed haloperidol in a network meta-analysis (Zhu et al., 2017), but this evidence came from low-moderate quality studies. Some second-generation antipsychotic medications (SGAs) have appeared to have lower rates of discontinuation than haloperidol (Zhang et al., 2013; Zhu et al., 2017) and low-quality evidence suggests that olanzapine and quetiapine may cause motor adverse effects less commonly (Zhu et al., 2017). Open effectiveness trials produce conflicting results (Crespo-Facorro et al., 2012; Kahn et al., 2008). Some of the findings in these studies may be explained if short-term motor adverse effects that are commoner with the older, first-generation antipsychotic (FGA) medications (particularly haloperidol) are more intrusive than the metabolic adverse effects commoner with SGAs, apart from weight gain. Metabolic adverse effects have a greater potential effect on long-term mortality (see below, Mortality cardiovascular disease and antipsychotic treatment) but in a national case register study of those starting antipsychotic medication for schizophrenia, with a follow-up duration of up to 11 years, no particular medication was shown to affect mortality differently (Tiihonen et al., 2009). Therefore, the choice of medication for adults of working age should be made with the patient largely on the basis of the known adverse effect profile.

Where the illness presents with significant agitation, aggression, anxiety, insomnia or distress it may be appropriate to favour an antipsychotic agent with substantial antagonism at $\mathrm{H} 1, \alpha 1$ or $5 \mathrm{HT} 2$ receptors as well as D2 antagonism to reduce arousal and provide sedation. When symptoms at presentation are very disturbing, clinicians might consider the balance of adverse effects presented by such agents against these temporary benefits. Similarly, they might balance the risks of temporarily dosing antipsychotic medications to reduce arousal rather than seeking the minimum effective antipsychotic dose. An alternative approach to increasing the dose of antipsychotic medication would be to add a GABA $\mathrm{A}_{\mathrm{A}}$ modulator (e.g. benzodiazepine) for a limited period. Of course, minimising antipsychotic dose increases the risk of an ineffective dose of antipsychotic medication failing to control the acute episode and requiring PRN ('as required') medication. Clinicians should consider all the circumstances of the illness, including how far the setting of treatment can mitigate the risks of poorly controlled early illness.

\section{Targets and trials of antipsychotic treatment}

Data from trials in more established illness indicate that a $25 \%$ reduction in total PANSS (Positive and Negative Syndrome Scale: Kay et al., 1987) score represents a meaningful minimum indication of response to an antipsychotic medication (Leucht and Kane, 2006), equivalent to a 1-point improvement on the Clinical Global Impression (CGI) (Guy, 1976), while 40-50\% represents a more convincing response equivalent to 2 points. There is evidence of a single underlying factor explaining $80 \%$ of the variance in improvement due to antipsychotic medication (Marques et al., 2014), suggesting that change in global score is a reasonable measure of efficacy, though these drugs have at best limited efficacy against cognitive impairment (Goldberg et al., 2007) and negative symptoms (Fusar-Poli et al., 2015). The most influential criteria for remission, rather than response, specify a rating of mild or lesser severity for a range of psychotic and negative symptoms (Andreasen et al., 2005).

Using standardised symptom scales to evaluate initial symptoms might allow for more rapid trials of antipsychotic monotherapy in clinical practice. The long-standing notion that acute psychotic illness shows a delayed response to antipsychotic medication has been challenged by data suggesting that more improvement is seen within the first 2 weeks than in any subsequent 2-week period, and the bulk of improvement over the first year occurs in the first month (Agid et al., 2003, 2006). Further, the lack of an early response to antipsychotic medication in the early weeks of treatment appears to be a robust predictor of subsequent non-response (Kinon et al., 2010; Samara et al., 2015; Stauffer et al., 2011). These findings may seem to call into question recommendations that an adequate trial of antipsychotic medication should last 4-6weeks although, in clinical practice, issues such as establishing the optimal dosage and medication adherence for a patient and the challenges of monitoring fluctuating and evolving symptoms mean that it is probably premature to declare failure after 2 weeks.

It also appears that reduction of symptom severity to meet the recognised remission criteria (Andreasen et al., 2005) is a viable treatment target. A median of $40 \%$ of patients sustained these criteria for 6 months in the observational studies systematically reviewed by Alaqeel and Margolese (2012), although the range was $17-78 \%$; studies conducted since have reported figures of 59\% (Gaebel et al., 2014) and 60\% (Chiliza et al., 2016). Moreover, a higher proportion of patients in each study achieved symptomatic remission at some point, rather than sustaining it 
until the final follow-up (e.g. $55-70 \%$ in several studies in the review, $91 \%$ in the study by Gaebel et al., 2014). The OPTIMISE study attempted to provide more precision (Kahn et al., 2018). Volunteers with first-episode schizophrenia (thus a selected sample, none detained) were treated in three phases: 4 weeks of amisulpride treatment, then randomisation to 6 weeks of olanzapine treatment or continued amisulpride treatment (masked from assessors), then a switch to 12 weeks of clozapine treatment. Participants progressed to the next phase only if they failed to 'remit' (i.e. reach the symptom criteria of Andreasen et al., 2005, though not sustained for 6 months). Of the 446 in the intentionto-treat sample, 33\% dropped out, 64\% remitted and 3\% completed all phases without remission.

Though disability, quality of life and risk of suicide or violence are equally, if not more important components of recovery, they are further from the mechanism of action of dopamine receptor antagonism and as such make less plausible target outcomes. Nevertheless, they are also generally better in those whose illnesses remit. Should social and interpersonal function, mood and quality of life all indicate that a patient has recovered, there is a case for accepting whatever the level of psychopathology is, but there must remain an elevated risk of deterioration. Of course, patients with capacity who are simply satisfied with their response without having reached remission remain the final arbiters.

Many of the studies reviewed assessed remission at least 6 months after treatment initiation and it seems that the illness will take longer to reach this threshold in some patients. At least 3 months may be needed to determine whether remission has been achieved with a particular antipsychotic agent (Agid et al., 2013). In the OPTIMISE study (Kahn et al., 2018), after 4 weeks of amisulpride $56 \%$ of the 446 participants in the intention-totreat sample remitted (67\% of completers) and 17\% dropped out, while in those 47 still unremitted and allocated to another 6 weeks of amisulpride, a further $45 \%$ of completers remitted. This suggests that the cumulative probability of remission in those who completed up to 10 weeks of amisulpride was $82 \%$, ignoring those who dropped out - though if none of those who dropped out remitted, as the authors assumed, the cumulative probability of remission went down to $60 \%$. Another consideration is that adjunctive interventions (e.g. psychological treatments, occupational and vocational interventions) may be initiated that require evaluation over longer periods, of the order of 6 months. Therefore, although remission may be a target, it may be reasonable in these circumstances to wait 6 months or more with a given antipsychotic medication, as part of a range of treatment interventions, to determine whether or not it can be reached.

\section{Antipsychotic dose reduction and discontinuation after the first-episode}

Overall, the evidence convincingly and consistently demonstrates that discontinuation of antipsychotic medication after a first-episode psychosis substantially increases the risk of symptom recurrence and relapse (Emsley et al., 2013a; Gaebel et al., 2011; Gitlin et al., 2001; Goff et al., 2017; Thompson et al., 2018; Zipursky et al., 2014), although there are no reliable demographic or clinical predictors of either relapse or continued remission (Bowtell et al., 2018). Even after a single psychotic episode, continuing antipsychotic treatment seems to reduce the risk of relapse by half, compared with the withdrawal of medication (Chen et al., 2010; Mayoral-van Son et al., 2016; Wunderink et al., 2007). Having conducted a meta-analysis of four RCTs and two non-randomised interventions involving medication discontinuation for first-episode psychosis, Zipursky et al. (2014) concluded that stopping antipsychotic medication should not be recommended if there is certainty about the diagnosis and no suggestion that medication side effects are contributing to problems with health or functioning.

Other observational evidence implies that a minority of patients may be able to discontinue antipsychotic medication successfully in the medium term. A first-episode, non-affective psychosis cohort in Denmark was followed-up after 1, 2, 5 and 10 years (Gotfredsen et al., 2017). Of those patients assessed at the $1-5$-year follow-ups, about $25 \%$ were remitted and not prescribed antipsychotic medication, with this figure rising to $30 \%$ after 10 years. Of those remitted without maintenance treatment at 5 years, $87 \%$ were also in that position after 10 years.

Trials provide a relatively unbiased estimate of the risks and benefits of discontinuation. The possibility has been raised that a proportion of patients whose first-episode has remitted may be candidates for discontinuation of antipsychotic medication in the medium to long term, at least within the context of a specialist first-episode service providing effective psychosocial interventions (Alvarez-Jimenez et al., 2016). According to such a view, the possible advantages for such patients might be the avoidance of adverse effects, improvement of the therapeutic relationship and better cognitive and social function (Omachi and Sumiyoshi, 2018; Wunderink et al., 2013). Relevant to such a view are the results of the MESIFOS study (Wunderink et al., 2007), which looked at outcomes for people with remitted first-episode, nonaffective psychosis, who had been assigned to either continuing antipsychotic medication or a guided reduction in dosage, with withdrawal if achievable. Of the 65 participants assigned to the guided reduction strategy, discontinuation of antipsychotic medication was achieved in $35(46 \%)$. This was maintained in 14 participants without relapse ( $22 \%$ of the discontinuation group) but medication was restarted in 16 because of relapse $(25 \%)$ and in the remaining five $(8 \%)$ because of symptom recurrence (five participants in the maintenance group also discontinued medication and this was restarted because of relapse in two). At the end of the trial, at 18-month follow-up, the proportion who had relapsed in the dose reduction/discontinuation group (43\%) was double the respective figure in the maintenance medication group $(21 \%)$. Over subsequent years, participants received 'treatment as usual' from their clinicians. When followed-up after 7 years (Wunderink et al., 2013), relapse had occurred in $62 \%$ of the participants in the initial dose reduction/discontinuation group, fewer than the $69 \%$ in the maintenance treatment group. Further, for those participants who had originally been assigned to the dose reduction/discontinuation strategy, the proportion with functional recovery $(46 \%)$ was more than double that seen in those originally allocated to maintenance treatment (20\%). These outcomes are difficult to interpret for two reasons. First, it is not clear how many in each group discontinued or substantially reduced medication doses, though after 7 years the proportions prescribed low-dose or no antipsychotic medication were $27 \%$ in the maintenance group and $38 \%$ in the discontinuation group. Second, the discontinuation group happened to function better from the outset (e.g. $54 \%$ in paid work rather than $36 \%, 37 \%$ 
with schizophrenia rather than $51 \%$ ), though measures related to these differences were included in an analysis that found the likelihood of good function was higher after guided reduction.

The findings of a similar study (Hui et al., 2018) add to the uncertainty about the possibility of any benefit from early drug dose reduction and withdrawal after first-episode psychosis. Patients with a first-episode psychosis that had shown resolution of positive symptoms were randomised in a 1-year, double-blind, placebo-controlled trial (Chen et al., 2010) to either early medication discontinuation or maintenance treatment with quetiapine $400 \mathrm{mg} /$ day. The proportion who relapsed with medication discontinuation (79\%) was, as in the Wunderink et al. (2007) RCT, double the figure in the maintenance treatment group (41\%). As in the Wunderink et al. (2013) study, the patients subsequently received naturalistic treatment but the follow-up period was longer, being 10 years. The main finding was that the early discontinuation of antipsychotic medication was associated with a higher risk of poor long-term clinical outcome, defined as persistent positive psychotic symptoms, being judged to require treatment with clozapine for treatment-resistant schizophrenia, or suicide. According to the investigators, the clinical implication of their findings was that for patients with a remitted first-episode psychosis, continuing antipsychotic medication for at least the first 3 years after starting treatment reduces the risk of relapse and the likelihood of a poor longer-term clinical outcome.

\section{Depot/long-acting injectable antipsychotic medication for first-episode schizophrenia}

National case register studies indicate that the proportion relapsing after starting depot/long-acting injectable (LAI) antipsychotic formulations is lower than for oral formulations (see Depot/long-acting injectable antipsychotic preparations): this is true after first hospitalisations too (Taipale et al., 2018a; Tiihonen et al., 2011). Two small, open randomised trials (Kim et al., 2008; Subotnik et al., 2015) found lower proportions relapsing on depot/LAI preparations than oral medication within 2 years of presentation. One open RCT, comparing oral and LAI risperidone within up to 3 years of presentation, found no difference in relapse rates (Malla et al., 2016) but another, larger, 2-year, raterblind, randomised comparison of paliperidone LAI with oral antipsychotic medications started within 5 years of presentation (Schreiner et al., 2015) found a statistically significant lower risk of relapse with the former.

Broadly good outcomes are consistent with evidence showing poor adherence to be a major predictor of relapse after first episodes of schizophrenia (See Adherence and antipsychotic medications, below) and adherence to be better with depot/LAI preparations (Salgueiro and Segarra, 2019; Taylor and Ng, 2013; Titus-Lay et al., 2018). Overall, it seems very plausible that in early illness, depot/LAIs prevent relapse better than oral formulations but limitations in study design and heterogeneity in samples and outcomes (Kishi et al., 2016) preclude a confident assessment of their place in therapy.

\section{Clozapine for first-episode schizophrenia}

Trials of clozapine (see also Clozapine section below) do not indicate substantial superiority over chlorpromazine as a first-line treatment (e.g. the double-blind RCT reported by Lieberman et al., 2003; Girgis et al., 2011). In the OPTIMISE study (Kahn et al., 2018), too few patients failed to remit after the first 12 weeks or so of antipsychotic treatment and then continue on to clozapine treatment to allow meaningful comparison of those patients starting clozapine after amisulpride and then olanzapine (i.e. third line) with those who started it after amisulpride alone (i.e. second line). Five of the 18 participants who completed the 12 weeks of clozapine treatment showed remission of their illness, and the mean improvement was substantial (mean reduction of 18.4 on the PANSS total score, close to the response to initial antipsychotic treatment) even though mean blood concentrations did not quite reach $0.35 \mu \mathrm{g} / \mathrm{mL}$, often taken as a necessary level to ensure an adequate trial.

Observational evidence indicates that rapid progress to a trial of clozapine is practicable and, in a study by Agid et al. (2011), efficacy was good, with a $75 \%$ response to clozapine. The findings of a systematic review and meta-analysis (Okhuijsen-Pfeifer et al., 2018) of the few relevant trials suggest that when used as a first- or second-line treatment in schizophrenia spectrum patients, clozapine outperforms other antipsychotic medications, bearing in mind that the additional professional input associated with the mandatory blood monitoring may contribute to the benefit seen. Nevertheless, these clinical trial findings are consistent with other observational data (Yoshimura et al., 2017) indicating that starting clozapine early is associated with about an $80 \%$ response rate compared with $30 \%$ when clozapine is initiated after a delay of 2.8 or more years. Moreover, Tiihonen et al. (2011) provided naturalistic data on the impact of clozapine maintenance treatment in their Finnish national case register study of patients discharged after their first hospitalisations for schizophrenia. Of the $58 \%$ of the cohort who continued on the same maintenance antipsychotic medication for more than a month, only those prescribed clozapine, or olanzapine, or depot/LAI antipsychotic medication were significantly less likely to be rehospitalised than those on the reference treatment of oral risperidone. Of course, allocation to treatment was not random but it seems unlikely that those prescribed clozapine or depot antipsychotics were selected as less likely to be rehospitalised.

The clinical implications are, first, that if remission has not been reached after adequate trials of two different antipsychotic medications, the diagnosis should be reconsidered and treatment adherence reviewed; CBTp and family intervention should be offered if available. Although operationalised consensus criteria for a minimum level of psychological intervention sufficient to identify treatment resistance have not been agreed (see Howes et al., 2017), these interventions are the best evidenced treatments for psychotic and overall symptoms and, as such, are supported by NICE Clinical Guidance (e.g. NICE CG178, 2014a). Second, while the definition of treatment resistance has been discussed at length (see Howes et al., 2017; Kane et al., 2019), in this context it essentially represents a failure to achieve the remission of psychotic symptoms, excitement or cognitive symptoms despite two adequate trials of antipsychotic medication reaching maximum tolerable dosage, at least at the mid-point of the licensed dose range shown in the Summary of Product Characteristics (SPC) or British National Formulary (BNF) (see section on Treatment-resistant schizophrenia below). Each trial should last at least 6 weeks for an oral antipsychotic or 3 months for a depot/LAI antipsychotic medication. If adherence to an oral 
antipsychotic cannot be assured, a depot/LAI antipsychotic should be considered. Lastly, clozapine should be considered in those who meet the above criteria for treatment resistance, particularly for those who also suffer distress or disability or present a risk.

\section{Recommendations for first-episode schizophrenia}

\section{First-episode psychosis diagnosis and assessment}

- Where the diagnosis of psychotic disorder is suspected in primary care, patients should be referred to secondary services as soon as practicable, with a delay of no more than a week if possible. (D)

- The initial assessment in secondary care should include appropriate history, mental state and physical examinations, and consideration of psychiatric and physical comorbidity (see Diagnosis and assessment section above). (S)

- The initial assessment should seek to exclude alternative diagnoses and this may include clinical appraisal of the initial course of psychosis without antipsychotic treatment for around 2 weeks in order to exclude ICD-10 F23 acute or transient psychotic disorders or (given abstinence from substance use) F1x.5 substance-induced psychotic disorders. This strategy should only be employed when risks such as aggression, self-harm, self-neglect, vulnerability or deterioration can be appropriately monitored and mitigated. In that case, $\mathrm{GABA}_{\mathrm{A}}$ modulating drugs such as benzodiazepines may be prescribed for anxiety or insomnia for up to 2 weeks before being tapered off. (S)

- Pre-treatment investigations should include electrolytes, liver function tests and an ECG to exclude congenital conduction abnormalities and the presence of a suspect Brugada syndrome ECG. Ideally, these investigations should be performed before the first prescription. Where this is not possible, consideration should be given to the choice of antipsychotic medications identified in relevant meta-analyses as less arrhythmogenic, for example with a relatively low affinity for hERG potassium channels and a low propensity to lengthen the QTc interval. Blood pressure, prolactin, lipids and blood glucose are important but less time-sensitive, though best assessed before first prescription to exclude any abnormality and allow assessment of any subsequent change due to potential adverse effects. Full neurological examination is required in the absence of neuroimaging (CT or sMRI) and of NMDA receptor autoantibody assays; thyroid function tests are also mandatory. (D)

- Secondary care multidisciplinary teams should make a psychiatric diagnosis but be prepared to revise it. (S)

- In circumstances other than an initial assessment of the course of illness for up to 2 weeks, to exclude transient or substance-induced psychotic disorders (in the absence of continued substance use), definitive treatment should begin as soon as possible in view of the appreciable risks of harm or deterioration that have been identified in this group. Where possible, antipsychotic medication should be selected for individual patients on the basis of likely adverse effects. Doses should in most circumstances be sufficiently low to minimise adverse effects, with effective doses often below conventional lower dose thresholds. (D)

\section{Treatment delay and refusal}

- For patients accepting only psychological treatment interventions, efforts should be made to persuade them to agree to a trial of antipsychotic medication. The effectiveness of treatment using psychological interventions in the absence of antipsychotic medication is uncertain and the risks of no treatment at all appear substantial. (C)

- It may be appropriate to detain patients for compulsory assessment of the response to medication in those circumstances where there are evident risks of immediate physical, interpersonal or social harm, but otherwise the risk of a poor response of psychotic or negative symptoms related purely to delayed pharmacological treatment is not predictable on an individual basis, so it may be appropriate rather to engage the patient in psychological treatment and monitor their progress. (S)

- When patients decline assessment and treatment despite efforts at engagement, given the risks of untreated illness it may be necessary to detain them to enable assessment and treatment. (S)

\section{Initial treatment with antipsychotic medication}

- Antipsychotic medication is the first line of treatment for psychosis. Initial doses should be below those typical in established illness but sufficient for efficacy, as demonstrated in studies of first-episode patients (see, for example, Kahn et al., 2008; Salimi et al., 2009; and Crespo-Facorro et al., 2012), that is, doses in the lower end of the licensed therapeutic range. (B)

- Differences in efficacy (rather than effectiveness) of antipsychotic medications are not well evidenced in first episodes of schizophrenia, though some agents (e.g. olanzapine, amisulpride, risperidone) might perform slightly better than others. Differences in adverse effects may well drive much of the observed differences in discontinuation. The adverse effect profile, including liability for sedation and adverse metabolic effects, should thus largely determine the choice of antipsychotic medication, in collaboration with the patient as far as possible. (S)

- Where there is risk, or distress, due to excitement, severe anxiety, hostility or insomnia, benzodiazepine or antihistamine drugs may be appropriate adjunctive agents. (S)

\section{Targets and trials of antipsychotic treatment}

- Trials of antipsychotic efficacy should be monitored using comprehensive symptom assessment scales such as the PANSS. Assessments should if possible be made at baseline and the end of a pre-determined trial period (usually 2 weeks initially). Trials of an initial dose may need to last only 2 weeks; total trial length for an agent at up to the maximum SPC or BNF dose may be around 6 weeks. (B)

- Sufficient response to initial treatment may be defined as a reduction of $25 \%$ in total score (e.g. within 2 weeks) on 
a symptom assessment scale or one point on the CGI point; $40-50 \%$ reduction or two CGI points indicates a definitive response (e.g. after 6 weeks). Remission of symptoms (for example, no more than a mild severity rating for a range of PANSS items, as indicated by Andreasen et al., 2005) is an appropriate target for overall treatment of the initial episode. (B)

- Where an antipsychotic medication has failed to achieve a satisfactory response after 6 weeks, or remission in conjunction with other interventions over a longer period, it is appropriate to switch to another antipsychotic agent and repeat the trial process. Since the evaluation of the impact of adjunctive psychological treatments may require 6 months or more, it may require months to determine that remission cannot be achieved. (D)

\section{Maintenance treatment and discontinuation}

- After remission has been achieved, maintenance treatment with antipsychotic medication at a standard dose will substantially reduce the risk of relapse for at least 2 years and is likely to be appropriate. (B)

- Patients should be offered the option of depot/long-acting injectable antipsychotic medication for maintenance treatment, given the evidence of lower risk of relapse. (B)

- Where remission has been maintained for more than 2 years after a single episode (i.e. proposed as Stage IIa schizophrenia; Scott et al., 2013), whether medication should be continued or withdrawn should be reviewed, unless low-grade psychotic symptoms indicate that the psychotic process is not fully abated (Stage IIc). (D)

- Decisions about the duration of maintenance treatment should be made jointly by the clinician and patient, considering the pros and cons of withdrawal versus continuing medication, including risk of relapse and medication side effects. The nature of the index episode (including speed of onset, insight, severity, and risk) can be helpful in assessing the risks associated with any future relapse. (S)

\section{Clozapine for first-episode psychosis}

- Where a patient's illness meets criteria for treatmentrefractory psychotic or disorganisation symptoms despite adequate trials of two antipsychotic medications, provision of family intervention and CBTp (where available), and review of diagnosis and medication adherence, a trial of clozapine should be considered. This point should be achievable within 1-3 years of initiating treatment. (D)

\section{Acute psychotic episode}

Whether because of selection for more refractory illness or some adverse effect of repeated episodes of relapse, the response of an acute episode to antipsychotic treatment appears to be poorer after the first episode and deteriorates as the illness progresses (Emsley et al., 2013b; Haddad and Correll, 2018). A meta-analysis of placebo-controlled acute treatment studies in established schizophrenia (Leucht et al., 2017) found that just over $50 \%$ of participants responded to antipsychotic treatment, defined as at least a $20 \%$ reduction in PANSS/BPRS scores ('minimally improved'), compared with a figure of $30 \%$ for those receiving placebo. The respective proportions for at least a $50 \%$ reduction in PANSS/BPRS scores ('much improved') were $23 \%$ and $13 \%$. As antipsychotic medications, despite differences in efficacy identified by network meta-analysis (Leucht et al., 2013), appear to have comparable effectiveness in large, naturalistic randomised, controlled, rater-blind trials (Cost Utility of the Latest Antipsychotic Drugs in Schizophrenia Study (CUTLASS); Jones et al., 2006) and double-blind RCTs (Clinical Antipsychotic Trials for Intervention Effectiveness (CATIE); Lieberman et al., 2005), the choice of drug should be guided by previous response and a patient's preference for medications of which they have experience or have adverse effect profiles they prefer. Clozapine remains the best option for treatment-resistant symptoms, despite the potential reduction in its relative efficacy with longer illness (see Clozapine section).

Generally, doses appear to be higher for the treatment of acute episodes in the context of established illness compared with first episodes (Haddad and Correll, 2018). Positron-emission tomography (PET) studies indicate that doses sufficient to occupy above about $60 \%$ of D2 receptors (e.g. Kapur et al., 2000; Kegeles et al., 2010; Uchida et al., 2011b) increase the likelihood of response. Moreover, it appears that striatal and pituitary D2 receptor occupancy exceeding around $80 \%$ (e.g. Kapur et al., 2000; Nyberg et al, 1995; Uchida et al., 2011b) is likely to cause parkinsonism, akathisia and hyperprolactinaemia. Avoidance of adverse motor effects and minimising lifetime exposure predicts a reduced risk of tardive dyskinesia (e.g. Carbon et al., 2017), as perhaps does using certain SGAs (Carbon et al., 2018). There is far less likelihood of avoiding metabolic adverse effects (e.g. weight gain) because these depend at least in part on the affinity for histamine H1, 5HT or other receptors and those antipsychotic medications with significant adverse effect profiles of this type have high affinity for these receptors and already occupy a high proportion before effective antipsychotic doses are reached (e.g. Correll, 2010). Although the dosage used for acute treatment might only be intended as temporary, with review during a shift to the maintenance phase, acute doses tend to be continued to minimise risk of relapse (see Antipsychotic dosage for maintenance treatment, below) so these remain relevant considerations. Minimum antipsychotic medication doses likely to be efficacious are identified by the BNF, but are discussed in more detail by Patel et al. (2013) and Leucht et al. (2014). Partial dopamine agonists are an exception to these considerations. For example, aripiprazole shows D2 occupancy exceeding $85 \%$ at the minimum therapeutic dose of 10-15 mg/day (Sparshatt et al., 2010). Above that dose there is little scope for increase in D2 occupancy and so limited advantage in terms of occupancy, effect or efficacy.

For acute psychotic episodes in established illness some of the same considerations apply as discussed in relation to dosing first-episode acute treatment. As far as possible, clinicians should seek a minimum effective dose, starting at the minimum dosage likely to produce a response (see BNF; Davis and Chen, 2004; Leucht et al., 2014; Patel et al., 2013) and increasing if there is no response (after 2 weeks, see below); and if necessary supplement antipsychotic medication in a time-limited way with a benzodiazepine or other $\mathrm{GABA}_{\mathrm{A}}$ modulator. There is a case for selecting an antipsychotic medication likely to reduce arousal and dosing judiciously, at least in the short term; moreover, in balancing the risks of prolonged acute illness against those of adverse effects, there is an argument that when the risks of continuing illness are 
high, clinicians should start with moderate doses, in order to avoid potential under-treatment in those few who have a relatively high-dose threshold for efficacy. In these uncommon cases, doses that are effective in $90 \%$ of a sample may be chosen rather than those effective in 50\% (Davis and Chen, 2004), though the risk of perhaps intolerable adverse effects is increased too and this distinction may be more extreme than the situation warrants. There is little evidence to support exceeding BNF maximum recommended doses or even maximum effective doses identified in reviews and meta-analyses of trials (e.g. Davis and Chen, 2004; Haddad and Correll, 2018; Kinon et al., 2008; Patel et al., 2013; Royal College of Psychiatrists 2014; Taylor et al., 2018), and certainly not unless blood concentrations are unexpectedly low on testing. High doses increase the risk of adverse effects such as parkinsonism and cardiac arrhythmias, the risk of the latter further increased by physical illness, abnormal electrolytes, or coprescribing of other psychotropics, diuretics or high-risk medications (Girardin et al., 2013; Vandael et al., 2017).

As described above in discussing first-episode treatment (see Targets and trials of antipsychotic treatment), initial response to treatment is predictive of later outcome in established as well as early psychosis (Leucht et al., 2005). Different trajectories of antipsychotic response have been identified (Marques et al., 2011) and in one study, $84 \%$ of the early nonresponders (defined as a failure to achieve a $20 \%$ reduction in total PANSS score at 2 weeks) remained non-responders after 3 months of treatment (Kinon et al., 2008). Thus, in clinical practice systematic assessment of symptoms at presentation and after 2 weeks should allow for a relatively robust prediction of treatment failure, with the implication that adherence and medication dosage should be reassessed if symptoms have reduced by less than $20-25 \%$ and a switch of antipsychotic medication or formulation considered. However, validated mental state rating scales are rarely used in routine clinical practice, limiting the applicability of this approach. For some insufficiently responsive illnesses, as doses edge higher, and/or in vulnerable populations such as adolescents, the elderly, pregnant women (see Westin et al., 2018), or those with liver or cardiac disease, there is potentially a case for measuring blood levels (at least of antipsychotic medications with reasonably well established blood concentration ranges, such as risperidone, olanzapine, amisulpride, haloperidol and clozapine; e.g. see Hiemke et al., 2017). Again, there is some logic in ultimately aiming for remission rather than response, as a viable target that predicts other advantageous outcomes and reduces the chance of deterioration.

There is no strong, consistent evidence for combining antipsychotic medications, for example, for refractory illness (e.g. Correl et al., 2009, 2017a; Haddad and Correll, 2018; OrtizOrendain et al., 2017; see also the section below on Combined non-clozapine antipsychotic medications (antipsychotic polypharmacy) for treatment-resistant schizophrenia) and a clear risk of toxicity from the combined dose (Barnes and Paton, 2011; Girardin et al., 2013). Existing guidelines such as NICE CG178 (NICE, 2014a) are clear on the need for psychosocial treatment such as CBT for psychosis and family intervention whenever possible. This will depend on what therapies patients have had before, how far they can be engaged and the resources available, but these are important interventions that may prolong an antipsychotic medication trial before teams can be sure that an episode is refractory to treatment (6 months; e.g. Howes et al., 2017). However, the evidence that psychosocial treatments alone are as efficacious as antipsychotic medication is weaker than the evidence in early illness (see Calton et al., 2008).

\section{Recommendations: Acute psychotic episode}

\section{Antipsychotic medication}

- Choice of antipsychotic drug should be based on the same criteria as suggested for first episode, but should additionally take into account:

- Any preference a patient may have for any particular antipsychotic medication. (S)

- A patient's past experience of individual antipsychotic medications in terms of relief of symptoms and side effects, including aversive subjective experiences. $(\mathrm{S})$

- Seek to conduct an adequate trial of the chosen antipsychotic medication in terms of dosage, duration (up to 4 weeks at optimum dosage) and medication adherence. (A)

- Antipsychotic dosage should be titrated against side effects and efficacy. (S)

- If starting an antipsychotic medication that the person has not previously been prescribed, the initial dosage should be at the lower end of the licensed range and slowly titrated upwards, if indicated, to the optimal range for that drug, and not exceeding the maximum licensed dose given in the BNF or SmPC. (S)

- The initial antipsychotic dosage regimen, including the speed of titration, should be consistent with the recommendations in the SmPC/BNF. (B)

- The justification for prescribed antipsychotic dosages outside the range given in the BNF or SmPC should be documented in the clinical records. (S)

- Regular combined antipsychotic medication should not be prescribed routinely, except for short periods when switching from one antipsychotic medication to another. (B)

\section{Medication regimen}

- Antimuscarinic agents should not be generally prescribed prophylactically with antipsychotic medication, but rather their use for emergent extrapyramidal problems responsive to such medication (e.g. parkinsonism and acute dystonia) should be determined on an individual basis, taking account of factors such as a patient's history of extrapyramidal side effects and the risk of antimuscarinic side effects. (S)

- The potential impact of comorbid substance use on the therapeutic efficacy and side-effect risk of antipsychotic medication should be considered. This should be discussed with patients and carers as appropriate. (S)

- Regular review of a patient's antipsychotic medication regimen should address the following: 
- Therapeutic efficacy, in terms of change in clinical domains such as symptoms, behaviour and cognition. (S)

- Side effects of treatment. (S)

- Medication adherence. (S)

○ Physical health. (S)

- The need to continue, change or stop medication, and the implications of the decision taken. (S)

- The requirement for PRN prescriptions of antipsychotic medication should be regularly reviewed in relation to the clinical indications, frequency of administration, therapeutic benefits and side effects, and cumulative dosage. (S)

\section{Maintaining response}

\section{Switching antipsychotic medication to enhance therapeutic response}

If a patient fails to respond to an adequate dose and duration of initial antipsychotic treatment, possible options are to increase the dose, add another antipsychotic medication or switch to another antipsychotic medication (other than clozapine), although there is no good quality evidence to help clinicians decide between these strategies (Samara et al., 2018). Switching is implemented more commonly in clinical practice than adding another antipsychotic medication (Bitter et al., 2008; Kahn et al., 2008; Kreyenbuhl et al., 2007; Weiden 2006). For example, in a 1-year, naturalistic study of outpatients with schizophrenia, Faries et al. (2009) found that antipsychotic medication was switched in approximately one-third. But when switching with the aim of enhancing therapeutic efficacy (Dold and Leucht, 2014), which antipsychotic medication should be chosen? Metaanalyses of RCTs reveal only modest differences in efficacy between the available non-clozapine antipsychotic medications and the clinical significance of any such small efficacy advantages is uncertain (Kishi et al., 2017a; Leucht et al., 2013).

Overall, the evidence suggests that switching antipsychotic medication in order to improve efficacy is likely to yield a clinically relevant improvement in only a small minority of patients (Kinon et al., 1993; Lindenmayer et al., 2002; Weiden, 2006), even at first episode (Agid et al., 2011, 2013; Kahn et al., 2018). Analysis of data from the CATIE schizophrenia trial (Lieberman et al., 2005) found that those participants randomised to a new antipsychotic medication showed no significant advantage in terms of symptoms, neurocognition, depression, quality of life, neurological side effects or costs, compared with those randomised to continue on the same antipsychotic medication (Rosenheck et al., 2009). The exception was that body weight increased in those randomly assigned to olanzapine, whether or not they had been receiving this medication before randomisation. The study by Faries et al. (2009), mentioned above, found that those patients who switched antipsychotic medication had poorer clinical and economic outcomes than those who remained on their medication regimens unchanged.

Despite the limited evidence for benefit, clinicians faced with a poor response to an initial trial of antipsychotic medication may consider that switching antipsychotic medication is a strategy worth trying (Suzuki et al., 2007). But they should take account of the risk of destabilisation of the illness and the provocation of adverse effects, including extrapyramidal side effects (EPS), following a switch (Essock et al., 2006). These are potentially attributable to discontinuation of the original medication, the extent of the response to the second antipsychotic, and/or differences between the pharmacological profiles of the two drugs (Lambert, 2007; Weiden et al., 2007). For example, if a patient is switched from an antipsychotic with high intrinsic anticholinergic activity to one with low activity there may be a greater risk of cholinergic rebound phenomena. To partly avoid such problems, a gradual cross-tapering of the dosages of the two antipsychotics is usually recommended rather than an abrupt switch (Ganguli et al., 2008; Lambert, 2007; Weiden et al., 2007), although there seems to be little empirical evidence to support any particular switching strategy (Remington et al., 2005; Takeuchi et al., 2008).

It remains uncertain whether the particular antipsychotic medications involved in a switch are relevant to the response, even though studies have hinted that a switch to amisulpride or quetiapine, or from risperidone to olanzapine (compared with olanzapine to risperidone), may achieve some clinical benefit (Hashimoto et al., 2015; Hatta et al., 2018; Kim et al., 2016). However, the antipsychotic formulation may have an influence; while switching from an oral antipsychotic to a depot/LAI may be beneficial (Schreiner et al., 2017), poorer outcomes have been reported for patients switching from a depot/LAI antipsychotic to oral antipsychotic medication (Barnes et al., 2013; Mustafa, 2017; Voss et al., 2015), which presumably partly reflects the opportunities for covert non-adherence with the latter as well as possibly the pharmacokinetic differences between oral and depot/ LAI preparations.

\section{Relapse prevention}

Definition and implications of relapse. Relapse may be considered as the return of the schizophrenia illness after partial recovery and can present with symptomatic, phenomenological and behavioural aspects. One issue affecting the interpretation of the evidence base for relapse prevention is the variation in the criteria for relapse employed in studies, some of which are unclear or of uncertain clinical relevance (Leucht and Kane, 2006). The focus on exacerbation of positive psychotic symptoms and hospital admission as defining features of relapse in such studies means that they have generally failed to address the impact of continuing, long-term antipsychotic treatment on other clinically relevant outcomes such as negative and affective symptoms, cognitive impairments, disability in social and occupational functioning and comorbid problems such as substance use, medical illness, and medication side effects.

Admission to hospital is the most commonly used proxy for relapse (Olivares et al., 2013), and while this is a pragmatic and intuitively meaningful definition, whether or not a patient experiencing a relapse is hospitalised may depend not only on the nature and severity of their symptoms and behavioural disturbance but also, critically, on a range of psychosocial and economic factors and the configuration and resources of the local clinical service.

A relapse of psychotic symptoms can be a distressing, disruptive and stigmatising experience, jeopardising personal relationships as well as education or employment status and carrying the 
risk of harm by patients to themselves or others. But relapse, as a period of active psychosis, may also be associated with disease progression. Follow-up studies have shown that greater frequency of relapse may be associated with poorer outcome and reduced responsiveness to antipsychotic medication (Curson et al., 1985b; Emsley et al., 2013b). Repeated relapse, often associated with poor medication adherence, seems to be associated with increased difficulty or delay in achieving remission (Leucht and Heres, 2006); about one in six patients in remission will not return to a remitted state following relapse (Emsley et al., 2013a; Ohmori et al., 1999). Such findings may partly reflect that relapse is more common in the context of a refractory illness with an inherently poorer prognosis. But they may indicate that following relapse, which is often a period of unchecked, untreated psychosis, recovery is compromised (Emsley et al., 2013a; Lieberman et al., 1996; Wyatt, 1997). Therefore, if relapse is associated with deterioration of the illness, at least in some patients, a critical question is whether a reduction in the frequency of relapse with continued antipsychotic medication can obviate such a decline and prevent or slow the development of refractory illness.

\section{Efficacy of continuing antipsychotic medication: Relapse}

prevention. There is consistent evidence from numerous clinical trials that continuing antipsychotic medication has a positive effect on the outcome of schizophrenia (Correll et al., 2018; Herold et al., 2017; Tiihonen et al., 2018). Such treatment can effectively reduce the risk of relapse two to threefold in the short to medium term (Correll et al., 2018; Davis, 1985; Davis et al., 1993; Gilbert et al., 1995; Kane, 2008; Leucht et al., 2003; Marder and Wirshing, 2003; 2012; Viguera et al., 1997). For example, Karson et al. (2016) conducted a systematic review of eligible studies reporting on the 'long-term' (1-2 years) effects of antipsychotic medication in people with first-episode psychosis and concluded that continuing on such medication had a markedly beneficial effect with regard to maintenance of remission and prevention of relapse. The NNT with antipsychotic medication to prevent one relapse over a 6-month to 1 -year period has been estimated to be between 3 and 5. The effect size for relapse prevention is one of the largest of all available medicines across all conditions (Leucht et al., 2012).

A meta-analysis of all RCTs of schizophrenia comparing withdrawal of chlorpromazine, one of the oldest antipsychotic medications, with continuing treatment with chlorpromazine (Almerie et al., 2008) found that, for people with established illness, withdrawal was associated with a significant increase in relapse in the short term (up to 8 weeks), medium term ( 9 weeks6 months) and long term (longer than 6 months). Leucht et al. (2012) reported a meta-analysis of data from 65 studies, conducted between 1959 and 2010 and involving 6493 participants, which had compared antipsychotic maintenance treatment with placebo, with a median follow-up of 26 weeks. Antipsychotic medication substantially reduced the risk of relapse in all patents with schizophrenia for up to 2 years of follow-up. The 1-year relapse figure for those patients whose antipsychotic medication had been stopped (64\%) was more than double that for those who had continued on medication $(27 \%)$. Relapse was characterised by a recurrence of positive symptoms and/or behavioural disturbance in the majority of cases. Takeuchi et al. (2017) compared the 1-year symptom trajectories of people with established schizophrenia receiving continuing antipsychotic with those assigned to placebo treatment in $11 \mathrm{RCTs}$, involving 2826 participants. Symptom severity remained stable in those participants continuing on antipsychotic treatment but gradually worsened in those switching to placebo.

While the heterogeneity of response to continuing antipsychotic medication is recognised, there seems to be little evidence to support the notion that a substantial minority of people with schizophrenia would maintain recovery indefinitely without such treatment (Carpenter, 2001; Gitlin et al., 2001; Wunderink et al., 2007). However, it must also be acknowledged that with longer-term treatment, the evidence for effective relapse prevention may be less robust. Analysing data from relevant studies, Leucht et al. (2012) found a statistically significant association between longer study duration and smaller relapse reduction by oral antipsychotic medication compared with placebo. While this has been interpreted as reflecting some loss of efficacy with longer-term treatment, the most obvious explanation is poorer medication adherence over time (Leucht and Heres, 2006; Valenstein et al., 2006). It might also be partly explained by the accumulation of systematic errors and bias in longer studies, and/or that patients with more chronic illness have a greater risk of relapse (Alphs et al., 2016). It has also been attributed to the breakthrough of psychotic symptoms related to antipsychotic-induced dopamine D2/D3 receptor upregulation and resultant supersensitivity. While preclinical studies demonstrate that such supersensitivity of postsynaptic striatal D2 receptors can be induced by long-term antipsychotic medication, the evidence from clinical studies is less clear-cut (Yin et al., 2017). Howes et al. (2012b) found limited evidence for a general D2/3 receptor upregulation in patients, although it may occur in some (Silvestri et al., 2000). Further, if dopamine hypersensitivity was responsible for the loss of long-term efficacy with continuing antipsychotic medication, then, as Correll et al. (2018) have speculated, the partial D2 agonist aripiprazole, which may not be associated with upregulation of dopamine D2 receptors, might be associated with a lower likelihood of relapse than dopamine D2 antagonist antipsychotic medications. But there is nothing to suggest that this is the case (El-Sayeh et al., 2006; Kane et al., 2009; Zhang et al., 2016).

The hypothesis of supersensitivity psychosis refers to a compensatory state of upregulation of postsynaptic D2 receptors which has been held to be potentially responsible for breakthrough psychotic relapse in people with schizophrenia on maintenance antipsychotic medication as well as rebound psychosis after abruptly stopping treatment (Chouinard, 1991; Chouinard et al., 2017; Kimura et al., 2016). Regarding breakthrough psychosis, a 20 -year, non-randomised, follow-up study found that patients with schizophrenia on continuing antipsychotic medication exhibited significantly more frequent 'psychotic activity' than those who were unmedicated (Harrow et al., 2014), a finding which the investigators partly attributed to the development of antipsychotic-induced supersensitivity of dopamine receptors or excess dopamine receptors. However, in a large, Finnish cohort of people with schizophrenia with 'virtually assured adherence' to their maintenance antipsychotic medication regimen, Rubio et al. (2019) found no convincing evidence of a causal relationship between cumulative exposure to antipsychotic medication and breakthrough of psychotic symptoms. Relapse was more likely in younger individuals early in their illness, and soon after treatment onset rather than later. 
If supersensitivity psychosis were a common consequence of abrupt drug withdrawal, this would undermine the validity of the evidence for relapse prevention that is derived from discontinuation studies, where relapse following withdrawal of medication is interpreted as a re-emergence of the illness. Clinical investigation of this issue tends to be confounded by the difficulty in distinguishing between the natural history of the underlying disorder and effects related to drug withdrawal, including cholinergic rebound symptoms. Nevertheless, this issue was addressed by post-hoc analysis of data from a relapse-prevention study in schizophrenia, which compared relapses in participants continuing on antipsychotic medication with those assigned to placebo (Emsley et al., 2018). The similarity of relevant aspects of relapse between the two groups led these investigators to conclude that relapses after treatment discontinuation reflected recurrence of the underlying illness, with little to suggest that withdrawalrelated phenomena had contributed to the high relapse rate after stopping treatment. Further, the observation by Takeuchi et al. (2017) that the symptom trajectories of patients following withdrawal of antipsychotic medication show a gradual worsening rather than an initial deterioration also fails to support the idea of a rebound supersensitivity psychosis. In addition, a meta-analysis of RCTs found no effect of abrupt or gradual withdrawal of antipsychotic medication on relapse risk (Leucht et al., 2012). Thus, the available evidence does not consistently support iatrogenic dopaminergic supersensitivity as a driver of psychotic relapse, either following discontinuation of medication or in patients receiving continuing antipsychotic medication.

Despite the common use of continuing antipsychotic medication in clinical practice, relapse rates remain relatively high. Although the majority of people starting antipsychotic medication for the first time will experience remission of symptoms within 3 months or so (Emsley et al., 2008; Lambert et al., 2008; Robinson et al., 1999; Üçok et al., 2011), a third or more of these patients will suffer a relapse of their illness in the first year to 18 months, and around $80 \%$ within 5 years (Csernansky and Schuchart, 2002; Prudo and Blum, 1987). Unfortunately, attempts to identify reliable clinical predictors of relapse or continuing remission among individuals who are prescribed continuing antipsychotic treatment have had only limited success. Commonly accepted predictors of relapse are poor medication adherence, male sex, a longer DUP, poorer premorbid adjustment, a greater severity of negative symptoms at baseline (Alvarez-Jimenez et al., 2012; Ascher-Svanum et al., 2006; Masand et al., 2009) and comorbid substance use (Buhler et al., 2002; Hunt et al., 2002; Linszen et al., 1994). While some of these risk factors are potentially modifiable (medication adherence, substance use and DUP), others are not (male gender and a poor premorbid level of functioning). More severe residual psychopathology and poor insight into the illness and the need for treatment have also been identified as risk factors but may be partly a proxy for poor medication adherence. A poor therapeutic relationship between the prescriber and patient and poor interaction between patients, their families and carers are also risk factors (Csernansky and Schuchart, 2002), presumably partly because a poorer therapeutic alliance has been consistently found to be associated with poor medication adherence (Lacro et al., 2002) and partly because environmental stress, particularly life events and high levels of expressed emotion within the family, are known to increase the risk of relapse. However, despite some understanding of the relevant factors, predicting which patients will be rehospitalised by clinical estimates or models is often no better than chance (Atakan et al., 1990; Olfson et al., 1999).

Choice of antipsychotic medication for relapse prevention. Only a relatively small number of longer-term, relapse-prevention trials comparing antipsychotic medications head-to-head have been conducted. The data remain insufficient to allow for any clear recommendation of superiority for any individual antipsychotic medication. A meta-analysis of data from 10 relapseprevention studies comparing FGAs and SGAs in people with established schizophrenia (Leucht et al., 2003) found a modest but statistically significant reduction in relapse rate with SGAs; the overall relapse rate with an FGA (haloperidol) was $23 \%$ while for SGAs the figure was $15 \%$. How far this possible advantage might reflect greater adherence to the latter medication remains uncertain. In practice, choice of maintenance antipsychotic medication for an individual patient may be more a question of the correct dose and formulation than drug group.

The real-world effectiveness of continuing antipsychotic medications was examined by Tiihonen and colleagues (2017), who analysed data from Swedish databases on rehospitalisation and treatment failure for adult patients with schizophrenia, between 2006 and 2013. There were 29,823 patients in a total prevalence cohort as well as 4603 in an incident cohort of newly diagnosed patients. Clozapine and depot/LAI antipsychotic medications emerged as being associated with the highest rates of prevention of relapse in schizophrenia, compared with no medication use. The risk of rehospitalisation was about $20 \%$ to $30 \%$ lower during depot/LAI antipsychotic treatment compared with equivalent oral formulations.

Two relatively long-term pragmatic trials of antipsychotic treatment (CATIE and CUTLASS1) may be relevant here. While they were not designed to address relapse prevention directly, the findings may be relevant to choice of antipsychotic treatment over the medium to longer term. Conducted in the US, the CATIE study (Lieberman et al., 2005) was a double-blind treatment study involving 1493 participants with established schizophrenia, which compared four SGAs and one FGA (perphenazine). All-cause discontinuation was the primary outcome measure and, over the 18-month follow-up period, the assigned study medication was discontinued in $60-80 \%$ of cases. Over any given period, there was a significantly lower chance of discontinuation for those participants assigned to olanzapine compared with the other antipsychotics being tested, but the discontinuation rate for perphenazine was similar to that seen with quetiapine, risperidone and ziprasidone. There were no differences between antipsychotic medications in the rates of emergent extrapyramidal side effects. Caveats relevant to the interpretation of the findings are that the maximum dose of olanzapine allowed was above the usual recommended upper limit. Weight gain and metabolic effects were more commonly linked with the discontinuation of olanzapine while perphenazine was more often discontinued because of extrapyramidal effects, despite participants with tardive dyskinesia not being assigned to perphenazine.

The Cost Utility of the Latest Antipsychotic Drugs in Schizophrenia Study (CUtLASS1: Jones et al., 2006) was a smaller, UK trial comparing randomised prescription of either an FGA or SGA (other than clozapine). The choice of individual medication was made by the managing psychiatrist. The study 
involved 227 participants with established schizophrenia for whom a change in antipsychotic medication was considered by their psychiatrist to be clinically indicated because of inadequate clinical response or intolerance. Over a 1-year follow-up period, there was no apparent disadvantage in choosing to prescribe an FGA (of which the most common was sulpiride) rather than an SGA in terms of quality of life, symptoms or the associated costs of care.

Antipsychotic dosage for maintenance treatment. The evidence for relapse prevention with antipsychotic medication derives largely from studies of antipsychotic medications within their recommended dosage ranges. There are no relevant highdose studies, with dosages above the recommended maximum. But there are a few clinical trials examining low dosage in firstepisode patients (e.g. Schooler et al., 2005), and some older studies testing low-dose FGAs, most of which used depot preparations in order to avoid covert adherence problems confounding the results (Hogarty et al., 1988; Johnson et al., 1987; Kane et al., 1983; Marder et al., 1984, 1987). A meta-analysis of six of these double-blind RCTs by Barbui et al. (1996) concluded that continuous low-dose treatment (defined as $50-100 \mathrm{mg}$ per day chlorpromazine equivalents) was less effective than standard dosage (200-500 mg per day chlorpromazine equivalents) at preventing relapse.

Kane et al. (1983, 1985) reported RCT findings comparing three different fixed doses of fluphenazine depot medication: a standard dose of $12.5-50 \mathrm{mg}$, a low dose of $2.5-10 \mathrm{mg}$, and a very low dose of $1.25-5 \mathrm{mg}$, each every 2 weeks. The cumulative relapse rate among those participants receiving the standard dose was $14 \%$, while the rates for the low and very low dosage treatment arms were $24 \%$ and $56 \%$, respectively. Similarly, Kane et al. (2002) also conducted a double-blind RCT comparing four different fixed doses (monthly injections of 25, 50, 100 or $200 \mathrm{mg}$ ) of haloperidol depot medication over 1 year. The usual maintenance dosage of haloperidol decanoate is $50-200 \mathrm{mg}$ every 4 weeks, and the participants assigned to $200 \mathrm{mg}$ a month showed the lowest rate of relapse or symptomatic exacerbation $(15 \%)$ with no substantial evidence that this dosage was associated with a greater risk of adverse effects or subjective discomfort, compared with the $100 \mathrm{mg}$ or $50 \mathrm{mg}$ dose groups. Nevertheless, the rates of symptom worsening with the $100 \mathrm{mg}$ $(23 \%)$ and $50 \mathrm{mg}(25 \%)$ dosage regimens were not statistically significantly greater than that observed with $200 \mathrm{mg}$ and the consequences of relapse in the context of this study were not judged to be generally severe.

Uchida et al. (2011a) considered that there were insufficient clinical trial data to allow for any firm conclusions to be drawn on the dosage of maintenance antipsychotic medication in schizophrenia and schizoaffective disorder. However, they conducted a meta-analysis of relevant studies, addressing the long-term efficacy of very low, low, and standard antipsychotic dose therapies for schizophrenia. There was a total of 1395 participants in the 13 double-blind RCTs, all of which had a follow-up period of at least 24 weeks. The efficacy of low- and standard-dose antipsychotic medication was comparable in preventing relapse in schizophrenia/schizoaffective disorders, although the definition of relapse varied widely across the studies. Nevertheless, these investigators concluded that treatment with less than half of the standard dose may increase the risk of treatment failure.
The results of these trials generally confirm the efficacy of standard dosages of antipsychotic medication for relapse prevention but leave the optimal dosage ranges for the available antipsychotics undetermined. While a consistent finding has been that a reduced dosage tends to be associated with an increased risk of relapse over time, some advantages with lower dosages have been reported, such as a lower incidence of extrapyramidal side effects.

\section{Continuous versus intermittent treatment}

Another clinical question is whether maintenance antipsychotic medication needs to be continuous. When a strategy of intermittent, targeted medication, with the provision of antipsychotic medication only when prodromal signs of relapse occur, was tested in clinical trials in the 1990s (Carpenter et al., 1990; Gaebel et al., 1993; Herz et al., 1991; Jolley et al., 1990; Schooler et al., 1997), it was found to increase the rate of prodromal symptoms, relapse and rehospitalisation and place a greater burden on families/carers while providing no consistent benefits in social functioning. Further, the resources required for the necessary close clinical monitoring were considered to be unsustainable.

Sampson et al. (2013) conducted a meta-analysis of 17 relevant trials of intermittent, targeted medication, with a total of 2252 participants and follow-up periods ranging from 6 weeks to 2 years. The main finding was that, in the long term, those participants assigned to intermittent treatment were more than twice as likely to relapse as those on continuing maintenance treatment. Another meta-analysis, by De Hert et al. (2015), included 10 eligible studies with 1230 participants. The findings were similar, with the risk of relapse of schizophrenia when exposed to 6 months of intermittent or placebo strategies being three times that with continuous treatment. Those participants assigned to continuous antipsychotic treatment had a lower risk of relapse and were free of relapse for longer.

Implementing an intermittent, targeted treatment approach depends upon the reliable identification of early warning signs of impending psychotic relapse. For many people with schizophrenia, the illness tends to deteriorate in a similar way in advance of a relapse, which in theory offers the opportunity for timely and effective intervention (Meyer et al., 2018). However, in practice, reliance on early warning signs may not be feasible, as such 'prodromal' signs (for example, anxiety and insomnia) appear to have only modest predictive validity for relapse, and the transition from remission to relapse may be abrupt with few or no such early warning signs or symptoms (Birchwood et al., 2000; Eisner et al., 2013; Emsley et al., 2013a; Gleeson et al., 2005). Whether a focus on 'basic symptoms' (Schultze-Lutter et al., 2007) would allow for a more reliable and potent prediction of relapse remains an area of uncertainty (Eisner et al., 2018).

\section{Depot/LAI antipsychotic preparations}

National register studies in Finland (Tiihonnen et al., 2006, 2017) have found that continuing treatment with depot/LAI antipsychotic preparations in clinical practice outperforms oral antipsychotic medication in preventing rehospitalisation. A meta-analysis of cohort studies found that treatment with depot/ LAI antipsychotic preparations was associated with a reduction in hospitalisations of $15 \%$ compared with oral antipsychotic 
medication, despite greater illness severity in those patients receiving the depot/LAI preparations (Kishimoto et al., 2018). But the key advantage of depot/LAI antipsychotic medications in clinical practice is the avoidance of covert non-adherence (Barnes and Curson, 1994). A lack of adherence to such a regimen, through failure to accept the injection, will be evident to the clinical team and thus allow the opportunity for appropriate, prompt intervention. Further, if administration has been documented there is the potential to clarify whether a poor therapeutic response represents treatment refractoriness rather than poor medication adherence.

The potential benefits of depot/LAI antipsychotic medication over oral preparations also include more consistent bioavailability, removal of the need to remember tablets, and regular contact with the healthcare professional administering the injection. However, the evidence from RCTs that these advantages translate into better global outcome or a reduced risk of relapse and hospitalisation is relatively thin (Haddad et al., 2009; Patel and David, 2005; Robert and Geppert, 2004). Kirson et al. (2013) conducted a meta-analysis of depot/LAI versus oral antipsychotic medication studies: five RCTs, four prospective observational studies, and four retrospective observational studies. The depot/LAI formulations displayed significant advantages in non-randomised observational studies, whereas in RCTs no difference was observed. Thus, the data on the relative efficacy of depot/LAI antipsychotic medication are conflicting and differ by trial design. While mirror-image studies and real-world cohort studies are more likely to show benefits for depot preparations, these observational studies have weaknesses. These include a lack of blinding, indication bias (the conscious choice of different treatments for patients with different prognoses) and more clinical contact for the patients prescribed depot/LAI medication than for those on oral antipsychotic medication and therefore a potentially greater level of support, more regular review of mental state and side effects, and enhanced adherence monitoring. Traditional RCTs tend to show equivalence between depot/LAI and oral antipsychotic preparations but these studies may also have intrinsic methodological elements which reduce their ability to identify differences between the two kinds of medication, not least being that they are generally relatively short term. Further, medication adherence by participants in a study is likely to be better than in clinical practice, partly because those patients consenting to participate in clinical trial are likely to be compliant with their treatment regimen (Bowen and Barnes, 1994). Further, hospitalisation during a trial may be uncommon because of the inherent scrutiny and level of support for participants in a research study.

Compared with oral antipsychotic medication, a key disadvantage of depot/LAI antipsychotic preparations is that steadystate plasma levels take considerably longer to achieve, so titration against response is a protracted process (Barnes and Curson, 1994; Kane et al., 1998). A clinician's selection of depot/ LAI antipsychotic medication for a particular patient should take account of differences between these preparations in terms of their individual pharmacokinetic profiles as well as their pharmacological and side-effect profiles (Citrome, 2013; Correll et al., 2016). Taking haloperidol decanoate as an example, the active drug is slowly released from the injection site, but not all will have been absorbed at the point the next due injection is given. The amount of active medication in the blood stream will therefore accumulate slowly over many weeks. Haloperidol decanoate plasma concentration will not reach steady state until an average of 15 weeks after any dosage change is made $(5 \times$ half-life of 21 days: haloperidol decanoate SmPC) and therefore the full effect of a dosage change cannot be systematically evaluated until this point. Thus, the clinical impact of a depot/LAI antipsychotic dosage change or change in injection interval, such as an increase in risk of relapse after a reduction in dosage, reduced risk of relapse following a dosage increase, or increased risk of EPS following a dose increase, may take months, perhaps years to be evident. Premature assessment of the impact of any dosage change may lead to unnecessary further dosage titration.

Clinicians commonly seek to remedy this reduced flexibility in dosage change with depot/LAI antipsychotic medications by adding an oral antipsychotic to the regimen, although this increases the risk of a, possibly inadvertent, high-dose prescription (Barnes et al., 2009). Another consideration is that formulations with a longer dosing interval have been introduced. These offer patients a reduced frequency of injections and allow more time for appropriate clinical intervention if injections are missed. But as Miyamoto and Fleischhacker (2017) point out, given the risk of relatively early dropout from depot/LAI antipsychotic treatment in the community (Adeosun, 2017; Olfson et al., 2007; Zhu et al., 2008), maintaining regular contact to ensure engagement of a patient in a therapeutic relationship with the prescriber and clinical team may be particularly relevant for successful treatment with depot/LAI preparations with a 3-month injection schedule (Berwaerts et al., 2015; Parellada and Bioque, 2016).

Clinicians may be reluctant to prescribe a depot/LAI antipsychotic preparation because they consider that this might compromise their therapeutic relationship with a patient. This is partly because of assumptions that depot/LAI antipsychotic preparations have a greater side-effect burden than oral antipsychotic medication, and partly because they are concerned that patients will perceive the injections as potentially stigmatising, intrusive or ignominious (Barnes, 2005; Heres et al., 2006; Parellada and Bioque, 2016; Patel et al., 2009). However, positive attitudes to depot/LAI injections are expressed by those patients already receiving depot/LAI preparations (Patel and David, 2005; Waddell and Taylor, 2009).

As the few direct comparison studies of depot/LAI antipsychotic preparations have not revealed much difference in terms of efficacy between those tested (Li et al., 2011; McEvoy et al 2014; Pandina et al., 2011), the nature of the side-effect burden is probably the primary criterion for choosing a particular medication. The adverse effects include uncomfortable local reactions at the injection site, characterised by pain, inflammation and induration, which have been estimated to occur in up to $15-20 \%$ of patients a year (Bloch et al., 2001; Hay, 1995; Jones et al., 1998), although lower rates with LAI SGAs have been reported more recently (Atkins et al., 2014; Jann and Penzak, 2018). Jones et al. (1998) suggested that the risk of such reactions with FGA depot/ LAIs might be minimised by the use of low-volume preparations and increasing the interval between injections.

\section{How long should maintenance treatment continue?}

The Schizophrenia Outpatient Health Outcomes (SOHO) study (Ciudad et al., 2008; Suarez and Haro, 2008) reported that of 
$4206(65 \%)$ of 6516 patients achieving remission with antipsychotic medication, $25 \%$ relapsed over a subsequent 3-year follow-up period. The constant rate of relapse may suggest that continuing treatment is required. But is it indicated for all patients with schizophrenia and for how long? Treatment guidelines generally recommend continuation of antipsychotic medication for every patient diagnosed with schizophrenia (Buchanan et al., 2010; Castle et al., 2017; Gaebel et al., 2005; NICE, 2009; Smith et al., 2009), suggesting that this is maintained for at least 2 years.

Rather than stipulate a period of time for which antipsychotic medication should be prescribed for all individuals with schizophrenia, clinicians should address the issue separately for each patient, reviewing treatment regularly and, at each review, considering the potential benefits and risks of continuing the medication regimen. The considerations include the awareness that the patient may not be adherent to the current treatment regimen (Coldham et al., 2002; Kamali et al., 2006).

While there are risks associated with the long-term use of antipsychotic medication, there are also risks associated with discontinuation, not least being a greater risk of relapse (Zipursky et al., 2014) and the adverse consequences of relapse, which include a hindering or reversing of any gains in social and vocational functioning that might have been made while receiving continuing treatment (Kam et al., 2015). While not all those who discontinue antipsychotic medication will experience relapse, there is a lack of reliable clinical and demographic predictors that would allow those individuals who are likely to be able to sustain remission after stopping medication to be identified (Bowtell et al., 2018).

\section{Recommendations for maintaining response}

- Established schizophrenia requires continued maintenance with doses of antipsychotic medication within the licensed range. Choice of antipsychotic medication should follow the respective recommendation for firstepisode schizophrenia (see above), but for each individual patient the following should also be taken into consideration:
- prior treatment response
$\circ$ experience of side effects
- level of medication adherence
comorbid physical illness. (S)

- As with acute treatment, each prescription of a maintenance antipsychotic medication should be an individual trial. (S)

- An oral antipsychotic medication regimen should be kept as simple as possible with respect to both the number of tablets to be taken and the number of times each day. (S)

- Before considering a switch in antipsychotic medication, the current antipsychotic medication should be optimised, and an adequate trial conducted in terms of dosage, duration and adherence. (S)

- The care plan should address remediable risk factors for relapse, such as comorbid substance use, poor adherence and a critical environment. (S)
- Any reduction in dosage of antipsychotic medication should be cautious and closely monitored, given the increased risk of relapse. (S)

- An intermittent, targeted treatment approach with antipsychotic medication should generally be avoided. (B)

- Depot/LAI antipsychotic formulations should be considered where the need to monitor medication adherence is a priority within the care plan or if the patient prefers the convenience of this approach. (B)

- Wherever possible, the prescriber should agree jointly with the patient on the choice of, and desired outcomes from pharmacological treatment and how these can be achieved. (S)

- The effectiveness of medication should be monitored and any identified side effects should be actively managed as appropriate. (S)

\section{Area of uncertainty}

There is a general lack of supportive evidence from high-quality trials for symptom improvement and treatment response in schizophrenia when continuing antipsychotic medication is augmented with a second antipsychotic medication (Galling et al., 2017; Ortiz-Orendain et al., 2018; see section below on Combined non-clozapine antipsychotic medications (antipsychotic polypharmacy) for treatment-resistant schizophrenia). Nevertheless, data from an observational study, involving all hospital-treated patients in Finland with schizophrenia, with up to 20 years of follow-up ( $n=62,250$, males $50 \%$, median age 45.6 years) suggest that antipsychotic polypharmacy may be superior to monotherapy for maintenance treatment and relapse prevention (Tiihonen et al., 2019). Co-prescription of more than one antipsychotic medication was generally associated with a slightly lower risk of psychiatric rehospitalisation than monotherapy, particularly combinations involving clozapine or a depot/LAI antipsychotic preparation. More specifically, the combination of clozapine and aripiprazole was associated with the best outcome: a 14-23\% lower risk of rehospitalisation than was seen with clozapine alone, which was the best outcome monotherapy. Further, secondary analyses on hospitalisation due to physical illness and mortality showed an advantage for antipsychotic combinations compared with monotherapy.

A critical confound in interpreting data from a real-world study such as this is indication bias. However, the investigators sought to minimise this by using within-individual analyses, with each patient used as their own control. Nonetheless, there are still several plausible explanations for the findings. It is possible that combining antipsychotic medications with different receptor profiles can be an effective pharmacological strategy and lead to better therapeutic efficacy and/or less of an adverse effect burden. Alternatively, in the context of poor medication adherence, co-prescription of two antipsychotic medications may increase the chance of a patient taking at least one. But also, as clozapine and depot/LAI antipsychotic preparations have been shown to be the most effective monotherapies for relapse prevention in the real world (Tiihonen et al., 2017), there is the possibility that attempting to mitigate metabolic side effects (e.g. by adding aripiprazole) or manage symptoms of agitation, anxiety or sleep disturbance (e.g. by 
adding olanzapine or quetiapine) keeps a patient engaged in their treatment, and therefore the bulk of the benefit seen with combined antipsychotic medication might actually be attributable to better adherence to the depot/LAI antipsychotic or clozapine treatment that has been augmented.

\section{Adherence to antipsychotic medication}

\section{Prevalence of poor medication adherence}

For any given medical disorder, the reported rates of poor medication adherence vary widely across studies. This partly reflects variation in the characteristics of the participants and methodological issues, including the definition of non-adherence (usually meaning that only some or none of the prescribed medication is being taken), the method of assessment and the duration of the study. Nevertheless, sub-optimal adherence to medication has been found to be common in all long-term disorders. In a metaanalysis across 17 non-psychiatric conditions, the reported prevalence of non-adherence ranged from $12 \%$ (HIV infection) to $35 \%$ (sleep disorders) with an average of $25 \%$ (DiMatteo, 2004). But medication non-adherence is a particular problem in people with schizophrenia, with prevalence at the higher end, if not even higher, than the range reported for non-psychiatric conditions (Kane et al., 2013). A comprehensive review by Lacro et al. (2002) reported a weighted mean prevalence of non-adherence in people with schizophrenia of $41 \%$ across 10 studies that met strict inclusion criteria. This increased to $50 \%$ when the analysis was restricted to five studies meeting a stricter set of inclusion criteria. While the level of medication adherence seems to be highly variable between individuals with schizophrenia, it may be relatively stable over time in the majority (Ascher-Svanum et al., 2006).

In a study of first-episode psychosis patients, Coldham et al. (2002), found that over half were poorly adherent or non-adherent within a year of starting treatment with antipsychotic medication. In an RCT involving first-episode patients with schizophrenia, schizophreniform disorder, or schizoaffective disorder, $29 \%$ of the participants discontinued antipsychotic medication against medical advice over a year (Perkins et al., 2008). Examining the medication records of people with established schizophrenia, Valenstein et al. (2006) found that $61 \%$ were partially adherent or non-adherent at some point during a 4-year period. Following discharge from hospital, up to $25 \%$ of patients with schizophrenia may be only partially adherent (Lam et al., 2003) and with continuing treatment, this figure may rise to $50 \%$ a year later and $75 \%$ by 2 years (Leucht and Heres, 2006; Mullins et al., 2008; Weiden and Zygmunt, 1997).

Although SGAs were introduced with claims of greater tolerability, their widespread use does not appear to have had any appreciable impact on the level of medication adherence in schizophrenia. There is little convincing evidence available to link the use of FGAs with poorer adherence, or conversely, the use of SGAs with increased adherence (Diaz et al., 2004; Lacro et al., 2002; Masand and Narasimhan, 2006), although some studies have suggested a modest advantage for SGAs (Al-Zakwani et al., 2003; García-Cabezaet al., 2001; Menzin et al., 2003; Tollefson et al., 1997).

\section{Factors influencing medication adherence}

Non-adherence is most commonly intentional, which may be defined as a patient deliberately deciding not to take medication for any reason, although a common explanation is that they consider that its disadvantages outweigh any benefits. Unintentional non-adherence occurs when an individual forgets to take medication or other factors interfere with medication taking (e.g. disorganisation symptoms, the medication is lost or not affordable or there is difficulty collecting a repeat prescription).

Medication adherence can be influenced by a variety of factors that include aspects of the illness being treated, the effects of the medication and the organisation of the responsible clinical service as well as attributes of the clinician, patient and caregivers (Haddad et al., 2014). Positive attitudes to medication and insight may be the factors most consistently associated with better adherence (Sendt et al., 2015; Velligan et al., 2009). A combined analysis of data from the CATIE study and the EUFEST showed that reduced adherence was associated with impaired insight, substance use and higher levels of hostility (Czobor et al., 2015). Negative patient attitudes to medication are associated with non-adherence (Brain et al., 2013; Yang et al., 2012). Other factors associated with poorer adherence to antipsychotic medication include negative attitudes towards medication from family members or peers (Brain et al., 2013) and the patient perceiving a poorer therapeutic relationship with their physicianprescriber (Day et al., 2005). Comorbid substance use is common in schizophrenia and its association with antipsychotic nonadherence is well documented (e.g. Novick et al., 2010).

Medication factors influencing adherence include dosing frequency, formulation, efficacy and side effects (Vergouwen et al., 2003). A meta-analysis of studies investigating the effect of dosing frequency on medication adherence in patients with chronic diseases showed better adherence for once-daily compared with more frequent medication regimens (Coleman et al., 2012). A similar finding was seen in a meta-analysis of patients with severe chronic psychiatric and neurological diseases including schizophrenia (Medic et al., 2013). Adherence may also be better when the medication has been associated with improvement in symptoms, particulary positive symptoms (Liu-Seifert et al., 2012). Further, the adverse subjective experiences related to medication may be more relevant to adherence than the severity of some other adverse effects (Lacro et al., 2002). Adherence may therefore be medication specific, where some medicines are taken regularly, others intermittently and others not at all. This is illustrated by a study by Piette et al. (2007) in people with schizophrenia and comorbid physical illness: data analysis that controlled for the average days of medication supplied and other regimen characteristics found that adherence rates were higher for antipsychotic medication than for antihypertensive or oral antidiabetic medication.

Attitudinal drivers of poor adherence. Specific drivers of poor adherence include neurocognitive deficits (in prospective memory, working memory, learning or comprehension), the lack of family or service support or reminders, and negative attitudes to medication (Abdel-Baki et al., 2012; Drake et al., 2015). The most important attitudinal predictors of poor adherence are a lack of perceived (or real) benefits from the treatment, the absence of a perceived risk of relapse, and a poor relationship between the 
patient and the prescriber, or perhaps key worker (Day et al., 2005). Possessing a high internal locus of control moderates the last of these, as such patients are less affected by external advice, so their internal attitudes to medication become relatively more important, as does an experience of control over management.

A psychological process of 'isolation' ('sealing over') can underpin these attitudes. Sealing over refers to a state in which a patient's self-concept and self-esteem depend on the belief that the threat to their well-being of a psychotic illness is essentially in the past (e.g. Lester et al., 2011; McGlashan and Carpenter, 1981; Tait et al., 2004). Accepting maintenance treatment with antipsychotic medication can undermine that. Thus, supporting a patient's transition to 'integration' (i.e. acknowledging an ongoing illness risk but maintaining good self-esteem based on a selfconcept that depends on managing it actively) might be an effective approach. While this has been shown to improve engagement rather than adherence (Hamann et al., 2007; Thompson et al., 2003), it is nonetheless plausible that preventing disengagement might be associated with improved adherence (Drake et al., 2015).

The acceptance of an illness label can predict a decline in self-esteem, at least in the short term, with the risk of depression and suicidality in early illness (Drake et al., 2015; Tait et al., 2004), possibly by undermining 'sealing over' as a coping strategy and encouraging feelings of shame, humiliation and being trapped by chronic disability (Iqbal et al., 2000). But observational studies of follow-up after first-episode, non-affective psychosis suggest that change in medication attitudes does not necessarily affect self-esteem (Drake et al., 2015). These findings support potential strategies for addressing these problems that include: developing a good therapeutic relationship between prescriber and patient; reinforcing medication as a solution to past problems and an insurance against future risk; and emphasising how the choice to adhere reflects maturity and accomplishment, rather than avoidance, but not highlighting diagnosis and illness. The diagnosis should be shared if directly requested by a patient (by analogy with the literature on breaking bad news such as cancer diagnosis, e.g. Faulkner et al., 1994) as otherwise productive discussion of prognosis or management options can be threatened. Doing this within the framework of CBTp may lead to an improvement in insight without engendering depression (Rathod et al., 2005).

Self-stigmatisation and the sense of a lack of control are often reported as problems by people with schizophrenia and they may impact adherence and engagement on the basis already discussed (Staring et al., 2009). A service ethos focussed on maximising information and autonomy for patients and family involvement has been proposed as an appropriate response and may improve adherence (Lacro et al., 2002; NICE, 2014a). There is observational evidence that sub-cultural stigmatisation of psychosis further predicts the attitudes of individuals (Corrigan et al., 2011; Evans-Lacko et al., 2012; Tsang et al., 2010). Work with families by culturally competent staff is a logical approach to this.

\section{Clinical consequences of poor adherence}

The clinical consequences of non-adherence with prescribed antipsychotic medication in people with established schizophrenia are considerable. It is the most common cause of relapse (Byerly et al., 2007; Fenton et al., 1997; Weiden et al., 2004) and associated with an increased difficulty or delay in achieving remission (Leucht and Heres, 2006). A 3-year, prospective, observational study of outpatients with schizophrenia conducted in various European countries found that antipsychotic nonadherence was significantly associated with an increased risk of relapse, hospitalisation and suicide attempts (Novick et al., 2010). Hospitalisation rates, as a proxy marker for more severe relapse, are increased in those patients who are non-adherent, with reported rates of $150 \%$ (Knapp et al., 2004) 200\% (AscherSvanum et al., 2006; Ward et al., 2006) and 400\% (Morken et al., 2008) over those found in patients who remain adherent. Further, non-adherent patients are more likely to use substances, be violent, be arrested (Ascher-Svanum et al., 2006), attempt or complete suicide (Herings and Erkens 2003; Novick et al., 2010; Tiihonen et al., 2006; Ward et al., 2006) and have poorer longterm functioning (Ascher-Svanum et al., 2006). An example of the benefits of good adherence comes from the CUTLASS study in the UK: analysis of the relevant data revealed that improved antipsychotic adherence was associated with improved quality of life over the course of a year (Hayhurst et al., 2014).

Even short periods of non-adherence can impact on treatment outcomes. In a US study, missing medication for 10 days was associated with an increased risk of psychiatric hospitalisation (Weiden et al., 2004), and the longer the gap without medication, the greater the risk of readmission. A patient's level of medication adherence prior to admission has also been shown to influence the subsequent inpatient course. Investigating a sample of patients with schizophrenia who had relapsed and been rehospitalised, McEvoy et al. (1984) compared those who had been adherent to their antipsychotic medication pre-admission with those who had not. The latter group had a more gradual onset of illness, were more likely to have required involuntary admission and had remained in hospital longer.

\section{Assessment of adherence}

Clinicians tend to underestimate non-adherence in their own patients (Byerly et al., 2005; Stephenson et al., 2012). If partial or erratic adherence is unrecognised or only suspected, clinicians may be uncertain whether a lack of adequate therapeutic response is due to limited efficacy of the medication, inadequate dosage or a refractory illness, potentially leading to unnecessary dosage increases, switching or polypharmacy (Velligan et al., 2003).

There are a number of ways to assess adherence to antipsychotic medication. Patients can be asked directly about how much of their prescribed medication they have taken over a specific period of time using non-judgemental questioning. There are also rating scales and checklists that have been developed to assess medication adherence, including the Medication Adherence Rating Scale (MARS; Fialko et al., 2008; Thompson et al., 2000), and the Brief Adherence Rating Scale (BARS; Byerly et al., 2008). In addition, there are scales that assess a patient's attitude to medication, such as the Drug Attitude Inventory (DAI; Hogan et al., 1983) and the Brief Evaluation of Medication Influences and Beliefs (BEMIB; Dolder et al., 2004), which may help identify those patients who are more likely to be non-adherent to medication.

Other indirect methods of assessing adherence include pill counts (Brain et al., 2014; Rosenheck et al., 2000), or prescription refill rates (Dolder et al., 2002). Sub-therapeutic antipsychotic 
plasma levels may be an indication of non-adherence (McCutcheon et al., 2018), but the range of plasma levels seen with the same antipsychotic dose in different patients means that these cannot reliably determine the level of adherence (Taylor et al., 2018).

Further technical solutions to assessing adherence include 'smart' or electronic pill containers such as the Medication Event Monitoring System (MEMS). The main drawback of such systems is that while they allow monitoring of the opening of a pill bottle, this does not necessarily equate with ingestion of the tablets. The use of such systems has largely been confined to research studies (Misdrahi et al., 2018; Nakonezny et al., 2008). Further, an electronic 'ingestible event marker' has been developed as an objective method to assess adherence (Kane et al., 2013), which involves embedding a nontoxic digital ingestion sensor in each tablet to be taken. So far, only a small feasibility study of this technology has been reported in patients with schizophrenia and bipolar disorder (Kane et al., 2013). The place of such technology in psychiatric research and clinical practice is unclear.

\section{Interventions to improve adherence}

The causes of non-adherence need to be understood for each individual patient and a customised management approach initiated: a range of interventions may be warranted. A Cochrane review (Nieuwlaat et al., 2014) of RCTs testing interventions to enhance medication adherence in both psychiatric and medical disorders found that effects were inconsistent between studies. The positive studies tended to involve complex interventions that could not easily be adopted in clinical practice. Given the disappointing outcomes of the overall adherence literature, there may be a need to apply the basic principles of good prescribing practice that might be expected to improve adherence in people with schizophrenia, such as shared decision making, avoiding the use of excessive doses, avoiding unnecessary polypharmacy and managing side effects. While the supporting evidence for a specific effect on adherence is limited, such practice is desirable for other reasons.

The quality of the relationship between a patient and their treating clinician or team is critical: good engagement and a strong therapeutic alliance increase the likelihood of good medication adherence (Macneil et al., 2009; Masand and Narasimhan, 2006), as does adequate social and family support (Rabinovitch et al., 2009). When patients perceive that they have been involved in the choice of medication they are significantly more likely to adhere to that treatment (Patall et al., 2008; Wilder et al., 2010). Ideally interventions should be patient specific, in that they target the barriers to treatment as perceived by the patient (NICE, 2009).

Adherence therapy, a development of what was originally called compliance therapy (Kemp et al., 1998), is a brief psychological intervention based on aspects of motivational interviewing and CBT. While this intervention showed some early promise for improving insight, adherence, attitudes towards medication and rehospitalisation rates in an inpatient sample (Kemp et al., 1996, 1998), further studies failed to replicate these positive findings for improving adherence (Barkhof et al., 2013; Byerly et al., 2005; O'Donnell et al., 2003), and a well-conducted, adequately powered RCT found adherence therapy to be ineffective in improving adherence, psychotic symptoms or quality of life in people with schizophrenia (Gray et al., 2006). Some evidence for efficacy has been provided by subsequent studies (Chien et al., 2019; Gray et al., 2016; Wong-Anuchit et al., 2019), although this generally relates to improving psychiatric symptoms rather than adherence directly.

For patients who are non-adherent because they simply forget to take their medication, practical solutions such as reminder charts or texts, or compliance aids consisting of medication containers with individual compartments that accommodate up to four doses of multiple medicines each day, may have a place. Electronic reminders have also been used to improve adherence (Montes et al., 2012; Vervloet et al., 2012). Currently such digital approaches have only been assessed in small studies in mental health, and their role in clinical practice remains unclear.

As already discussed (see section on Depot/long-acting injectable antipsychotic preparations), an alternative strategy to tackle poor adherence is the use of depot/LAI antipsychotic preparations, which make adherence transparent. Thus, a trial of guaranteed medication delivery with a depot/LAI preparation can help differentiate a treatment-resistant illness from poor adherence. Theoretically, depot/LAIs would be expected to improve unintentional non-adherence as they reduce the practical difficulties associated with taking regular daily medication regularly. As an individual only has to make a decision on whether or not to take medication once every injection cycle they could also improve intentional non-adherence at least in some people. Observational studies provide some evidence to support improved adherence with depot/LAI antipsychotic medications in the form of longer periods until treatment discontinuation compared with the comparable oral antipsychotic medication (e.g. Tiihonen et al., 2011; Zhu et al., 2008).

Nevertheless, a proportion of patients do miss or discontinue depot injections. With the older preparations there are reported non-adherence rates of $18 \%$ over a 3 -year period (Johnson and Freeman, 1973) and 40\% over a 7-year period (Curson et al., 1985a). There is no evidence that the attrition rate from SGA LAIs is lower than that with FGA depot preparations, though only a few studies have compared two depot/LAI antipsychotic medications (Nielsen et al., 2015; Stone et al., 2018). In UK mental health services, depot/LAI antipsychotic preparations have been prescribed for a quarter to a third of patients with schizophrenia, depending on the clinical setting (Barnes et al., 2009), but there is some evidence to suggest that, in practice, their use is not always targeted towards poorly adherent outpatients (West et al., 2008). The decision to use a depot/LAI should be made on an individual patient basis and the patient fully involved in the decision-making process.

\section{Recommendations regarding medication adherence}

- Where possible, offer a choice of antipsychotic medication, based on the known relative liability for adverse effects. Take into account the known adverse effect profiles of individual antipsychotic medications, a patient's past experience of adverse effects, the risk of drug interactions, and past medical history. (S)

- Where possible, the prescriber should agree jointly with the patient on the choice of medication, the desired outcomes, and how these can be achieved. (S) 
- The medication regimen should be kept as simple as possible with respect to both the number of medications to be taken and the number of times each day. (S)

- The effectiveness, tolerability and safety of a medication regimen should be monitored and any identified side effects should be actively managed as appropriate. (S)

- The patient should be asked at regular intervals in a nonjudgemental manner how much of their prescribed medication they have taken recently and their views sought regarding the efficacy and tolerability of this medication. (S)

- Consideration should be given to using one of the validated rating scales or checklists to assess a patient's attitudes towards medication. (D)

- In patients with a history of non-adherence leading to relapse, consideration should be given to using more objective methods to monitor adherence to oral medication regimens such as pill counts and, for some antipsychotic medications, plasma drug levels. (S)

- A depot/LAI antipsychotic formulation should be considered when this is preferred by the patient, previous nonadherence has led to frequent relapse, or the avoidance of non-adherence is a clinical priority. (S)

- Interventions to improve medication adherence should be patient specific, in that they should target the barriers to achieving adherence as perceived or noted by the clinical team to be present in that patient. (S)

\section{Addressing poor adherence after first-episode psychosis}

- Adopt an active service ethos with family engagement and early family intervention (NICE, 2014a), which can improve medication adherence (Pharoah et al., 2010) and service engagement. (B)

- Multidisciplinary teams should assess patients' attitudes to treatment and the value of medication and should consider steps to ameliorate poor adherence. They should assess feelings of shame, humiliation and entrapment and consider how to address isolation and integration, as these affect engagement and consequently adherence. Such assessments are particularly relevant when patients are stepping down care (e.g. being discharged from inpatient units) because of the likelihood of poor medication adherence at this juncture. Arrangements for immediate follow-up may potentially mitigate increased suicide risk and improve medication adherence (Kam et al., 2015; Upthegrove et al., 2010, 2014). (S)

- Depot/LAI antipsychotic preparations are a treatment option to consider where poor concordance or adherence to continuing oral antipsychotic mediction has been identified. (B)

\section{Key uncertainty}

There is evidence from controlled trials across a number of disease areas that suggests that financial incentives may have some potential to enhance medication adherence. An RCT conducted in the UK provided some evidence that a small financial incentive can improve adherence to depot/LAI antipsychotics over a year in people with schizophrenia (Priebe et al., 2013). But when the trial ended, and the incentive was withdrawn, adherence returned to the baseline level (Priebe et al., 2016). While such an approach raises ethical issues (Claassen, 2007), it may warrant further research.

\section{Mortality, cardiovascular disease and antipsychotic treatment}

\section{Mortality in schizophrenia}

There is consistent evidence that people with schizophrenia have an elevated mortality risk, two to three times that of the general population (Brown et al., 2010; Hoang et al., 2011; Lawrence et al., 2013; Saha et al., 2007). Thus, there is a mortality gap of 6-25years for schizophrenia versus the general population (Brown et al., 2010; Crump et al., 2013; Lawrence et al., 2010; Tiihonen et al., 2009). Correll et al. (2017b) concluded that this mortality gap between schizophrenia and the general population has been widening across the continents and this is in accord with the findings of other research groups (Hjorthøj et al., 2017; Hoang et al., 2011; Lahti et al., 2012; Nielsen et al., 2013a, Saha et al., 2007) including groups in the UK (Hayes et al., 2017; Smith et al., 2013), although there is some uncertainty as to whether this gap continues to widen (Tanskanen et al., 2018).

Although Schneider-Thoma et al. (2018) addressed shortterm mortality in a meta-analysis of RCTs comparing SGAs with placebo, there are no large, long-term RCTs providing data on antipsychotic medication and mortality. Therefore, large prospective cohort studies, including linkage studies, afford the highest level of available evidence. Attention to methodological limitations is required particularly for survival bias (those still alive when entering the follow-up phase may be more resistant to fatal risk factors), limited data collection on known confounders (socioeconomic and lifestyle factors, schizophrenia illness severity, substance use), cohort effects and confounding both by indication and contraindication (De Hert et al., 2010; Ghaemi and Thommi, 2010; Torniainen et al., 2015; Weinmann et al., 2009). While causality cannot be directly inferred from observational studies, a growing body of evidence has increasingly converged on some key aspects.

Major natural causes of death in the general population are cardiovascular, cerebrovascular and respiratory disease as well as cancer, and the same is true for people with schizophrenia. Reductions in mortality due to cardiovascular and respiratory disease have been seen in the general population but not in schizophrenia (Lawrence et al., 2010; Saha et al., 2007). One review of the relevant literature concluded that over two-thirds of people with schizophrenia die of coronary heart disease, compared with about half of the general population (Hennekens et al., 2005). Relevant risk factors, many of which are inter-related, include cigarette smoking, diet, exercise, obesity, relative poverty and poor healthcare (Barnes and Kerwin, 2003; Hennekens et al., 2005; McCreadie et al., 2003). Alternatively, Morden et al. (2012) reported that mortality rates for cardiovascular disease (CVD) were largely similar for people with schizophrenia compared with people without serious mental illness in a large-scale Veterans Affairs registry study. For cancer, mortality rates have been reported as higher in both men and women with schizophrenia than in the general population, perhaps partly related to the condition being under-detected rather than under-treated (Crump et al., 2013). 
Weinmann et al. (2009) conducted a systematic review of 12 studies including five small prospective cohorts and concluded that the data available provide some support for the hypothesis that long-term exposure to antipsychotic medication increases mortality in schizophrenia. Subsequently, large prospective cohorts have revealed antipsychotic use is associated with a lower mortality rate compared with no antipsychotic use (Baandrup et al., 2010; Crump et al., 2013; Tiihonen et al., 2006, 2009, 2011). Torniainen et al. (2015) conducted a Swedish population-based prospective cohort linkage study with 5-year follow-up for 21,492 people with schizophrenia, each with 10 population controls matched by age and gender. They found that no antipsychotic dose exposure was associated with the highest overall mortality (hazard ratio $\mathrm{HR}=6.3$, 95\%CI, 5.57.3 , defined daily dose $\mathrm{DDD} /$ day $=0$ ), followed by high-exposure $(\mathrm{DDD} /$ day $>1.5, \mathrm{HR}=5.795 \% \mathrm{CI}, 5.2-6.2)$, low-exposure $(\mathrm{DDD} /$ day $<0.5$, HR=4.1 95\%CI, 3.6-4.6) and then moderateexposure $(\mathrm{DDD} /$ day $=0.5-1.5, \mathrm{HR}=4.095 \% \mathrm{CI}, 3.7-4.4)$ groups. The authors suggested that their results demonstrated that excess overall mortalities are attributable to factors other than antipsychotic treatment when used in adequate doses. They further reported that both the no-exposure and high-exposure groups were associated with higher cardiovascular mortality as well as higher cancer mortalities than the low-exposure and moderate-exposure groups, and these effects are discussed in more detail below. However, for respiratory mortalities the rate increased by degree of exposure and was highest in the highexposure group $(\mathrm{HR}=18.5,95 \% \mathrm{CI}, 13.9-24.6)$. In a related analysis of the same cohort, Tiihonen et al. (2016) reported that exposure to benzodiazepines showed a dose-response relationship with mortality (HR up to $1.7,95 \% \mathrm{CI}, 1.5-2.0$ ). Tiihonen et al. (2009) conducted a Finnish population-based prospective cohort linkage study with an 11-year follow-up for people with schizophrenia treated with antipsychotic medication and also found that long-term treatment was associated with a lower mortality compared with no antipsychotic drug use. Patients on continuing clozapine treatment seemed to have a markedly lower mortality, while for those prescribed other SGAs it was neutral or increased.

In order to address survival bias, Torniainen et al. (2015) also analysed a cohort of 1230 first-episode patients and observed the highest excess overall mortality in those not receiving antipsychotic medication $(\mathrm{DDD} / \mathrm{day}=0, \mathrm{HR}=9.9,95 \% \mathrm{CI} 5.9-16.6)$. In a first-onset schizophrenia cohort study with a 5-year follow-up $(n=6987)$, clozapine, olanzapine and quetiapine were associated with reduced overall mortality rates whereas several FGAs were associated with increased mortality rates (Kiviniemi et al., 2013).

For unnatural causes of death, suicide rates have been found to be higher in schizophrenia than in the general population (Crump et al., 2013; Palmer et al., 2005; Qin and Nordentoft, 2005), and thus also contribute to the mortality gap, albeit to a lesser extent than death by natural causes (Dutta et al., 2012; Nielsen et al., 2013a). In the study by Torniainen et al. (2015), mortality by suicide was highest for the no antipsychotic exposure group (HR: 10.9, 95\% CI, 6.8-17.5) and lowest for the highexposure group (HR: 6.68, 95\%CI, 4.7-9.4). In the related analysis by Tiihonen et al. (2016), exposure to benzodiazepines had a dose-response relationship with mortality by suicide. There is evidence to suggest that clozapine is associated with a reduction in mortality by suicide (Kiviniemi et al., 2013; Meltzer and
Baldessarini, 2003; Ringback Weitoft et al., 2014; Saunders and Hawton, 2009); whether this may be partly attributable to better adherence to a clozapine treatment regimen is uncertain (Ringback Weitoft et al., 2014).

\section{Cardiovascular disease deaths in schizophrenia and antipsychotic medication}

As outlined above, death from CVD is a major contributor to the increased mortality rate compared with the normal population (Hennekens et al., 2005). Correll and colleagues conducted a large-scale meta-analysis of published studies investigating 3.2 million patients with severe mental illness (SMI) and 113.4 million controls (Correll et al., 2017b) and confirmed this finding. In longitudinal studies of schizophrenia, the significantly increased adjusted (for a mean of six confounders) HRs for CVD was 1.95 and for death due to CVD 2.45. The HRs for coronary heart disease (CHD), and for cerebrovascular disease were similarly elevated at 1.59 and 1.57 , respectively. Similar data have been found in the UK, with increased mortality particularly noticeable in the young and middle-aged groups (Osborn et al., 2007). These investigators used the GP Research Database and studied 46,136 people with SMI and 300,426 without SMI. HRs for CHD mortality in people with SMI compared with controls were 3.22 (95\%CI, 1.99-5.21) for people 18-49 years old, 1.86 (95\%CI, 1.63-2.12) for those 50-75years old, and 1.05 (95\%CI, 0.92-1.19) for those older than 75 years of age. For stroke deaths, the HRs were 2.53 (95\%CI, 0.99 6.47 ) for those younger than 50 years, 1.89 (95\% CI, 1.50-2.38) for those $50-75$ years old, and 1.34 (95\% CI, 1.17-1.54) for those older than 75 years.

There are multiple factors underlying the increased cardiovascular morbidity and mortality in SMI and in schizophrenia in particular, but here the focus will be on the role of antipsychotic medication. A non-systematic review concluded that, despite the obvious risks of metabolic side effects, antipsychotic use has not been shown to consistently increase CVD morbidity or mortality in schizophrenia (Ringen et al., 2014). In a recent meta-analysis of drug trials with placebo controls, use of antipsychotic medication was not found to be related to increased mortality in schizophrenia, although the higher general mortality in this patient group was replicated (Khan et al., 2013). A Swedish study showed that those patients with schizophrenia (including firstepisode patients) who were unmedicated or prescribed high-dose antipsychotic medication had the highest overall CVD mortality (Torniainen et al., 2015). Similarly, Vermeulen and co-workers (2017) found that no exposure to antipsychotic medication was associated with a greater risk of cardiovascular mortality than observed with long-term treatment with low to moderate dosage of antipsychotic medication, although not with high-dose treatment. Correll and colleagues found that CVD incidence increased with higher antipsychotic use, higher BMI and baseline CVD (Correll et al., 2017b). However, Osborn and co-workers concluded that the increased CHD and stroke mortality is not wholly explained by antipsychotic medication, smoking or social deprivation scores. Interpretation of the relationship observed between antipsychotic dose and CHD mortality should take account that the use of higher dosage may be an indicator of more severe illness which might impact on mortality through other lifestyle factors (e.g. smoking) (Osborn et al., 2007). 
Using UK databases, Brauer et al. (2015) reported an increased risk of death from myocardial infarction in the first 30 days after the initiation of both FGAs and SGAs that was not attributable to any other obvious factor. This finding highlights the need for vigilance in the early phase of antipsychotic medication use (as addressed further below).

\section{Antipsychotic medication and cardiac electrophysiology}

Antipsychotic medications have a quinidine-like effect on cardiac electrophysiology (Buckley and Sanders, 2000; Fayek et al., 2001; Johannesen et al., 2014; Yap and Camm, 2003). One of the main effects is a dose-dependent delay to ventricular repolarisation which is mediated by the delayed rectifier $\mathrm{K}^{+}$channel, encoded by the hERG gene. The effect is manifest on the ECG as $\mathrm{T}$ wave abnormalities, prominent $\mathrm{U}$ waves and QT interval prolongation. The QT interval must be corrected for heart rate (QTc) and the correction discussed here is the QTc calculated by Bazett's formula. QTc prolongation is associated with increased rates of torsade de pointes (TdP). This is a polymorphic ventricular tachycardia which typically manifests itself in convulsions, dizziness and syncope and which can also lead to ventricular tachycardia (VT) and ventricular fibrillation (VF). TdP is known to be a predictor of sudden death especially in patients with cardiac disease. This is an entirely separate mechanism from the primary pharmacological actions of antipsychotic medication and therefore a risk of arrhythmia is entailed which is not associated with any clinical advantage (Nielsen et al., 2011) The QTc interval is considered to be prolonged if greater than $440 \mathrm{~ms}$ for men and $470 \mathrm{~ms}$ for women, and a QTc over 500 ms should provoke considerable concern and prompt immediate cardiac review (Lambiase et al., 2019). A prolonged QTc may be familial and, along with the other adverse outcomes mentioned above (TdP, VT, VF), it is increased in prevalence by factors such as electrolyte imbalance (especially low potassium), liver disease, anorexia, alcohol use, some central nervous system disorders, female sex and a wide variety of cardiovascular disorders. Pharmacokinetic drug interactions, resulting in increased plasma or tissue concentrations of a QT interval-prolonging drug, are another risk factor for TdP. The most common interaction is via inhibition of the hepatic cytochrome P450 isoform CYP3A4. This isoform is important because it is very abundant in the human liver and is primarily responsible for the metabolism of many QT-prolonging drugs. It is important to note that some hepatic enzyme inhibitors (for example, erythromycin and ketoconazole) also delay cardiac repolarisation in their own right and this effect increases the severity of the interaction (Vandael et al., 2017). There is evidence that TdP most often occurs early during therapy, which has implications for monitoring as it is logical to focus this over the period of highest risk (Cubeddu, 2016). The risks of QT prolongation and of TdP with antipsychotic medications have been reviewed (Beach et al., 2018; Sicouri and Antzelevitch, 2018). Thioridazine and intravenous haloperidol remain the agents most associated with QTc prolongation and risk, although the latter's risk is confounded by its use in very ill patients. Pimozide, sertindole, amisulpride and ziprasidone also appear to be among the antipsychotic medications most likely to prolong the QTc interval (Huhn et al., 2019; Lambiase et al., 2019).
Recently a 'hERG safety margin' has been proposed in which the half-minimal inhibitory concentration of drugs from a hERG assay is divided by the peak human plasma concentration (Cmax) (Hazell et al., 2017). A hERG safety margin (hERG IC50/Cmax) of under 30 is generally predictive of medications with a high liability for TdP. For antipsychotic medications it is less predictive, but it identifies clozapine, droperidol and thioridazine as high risk and quetiapine, lurasidone (Greenberg and Citrome, 2017), cariprazine (Lee et al., 2019) and ziprasidone as low risk, with intermediate results for haloperidol, olanzapine and risperidone. Amisulpride and chlorpromazine have hERG safety margins of greater than 30 but a slight liability for TdP. Thus, it seems likely that the arrhythmogenic properties of antipsychotics are multiple in origin rather than directly linked to their effects on repolarisation (see also below). Further research on this measure is required as it may be the basis of screening for drugs with a high risk of cardiac rhythm disorders.

A further effect of antipsychotics on the ECG is linked to their propensity for sodium channel blockade and is seen by the presence of broad QRS complexes on the ECG. Sodium channel blockade is also associated with ST elevation in R precordial leads (Brugada-type ECG). The Brugada-type ECG increases the chances of arrhythmia/sudden death especially during periods of myocardial ischaemia. Blom and colleagues (Blom et al., 2014) showed that a Brugada-type ECG was present in $11 \%$ of a large sample of people with established schizophrenia, compared with $1-2 \%$ of aged-matched controls. However, it was also found in $9 \%$ of the patients who were not receiving antipsychotic medication with sodium channel blocking propensity, and therefore the reported high prevalence of Brugada-type ECG in schizophrenia, and the associated risk, may not be solely attributed to the use of sodium channel-blocking antipsychotics. The increased prevalence of this ECG abnormality in schizophrenia and related conditions has recently been reported by Sutterland et al. (2019), who also found a lack of association with antipsychotic use, highlighting the importance of carrying out an ECG prior to treatment (see also section on First-episode psychosis above).

\section{Sudden death}

Antipsychotic medication has therefore at least two electrophysiological effects which might be associated with sudden death in particular and CVD mortality in general: prolonged QTc and the Brugada-type ECG. Some studies have shown increased rates of sudden death in schizophrenia and an association with antipsychotic medication (Ray et al., 2009). There are two, key UK studies. Reilly and colleagues (2002) examined 69 cases of sudden death occurring over a 12-year period in five large mental hospitals. Thioridazine use was strongly associated with sudden deaths with a HR of 5.3, and this finding was partly responsible for the subsequent restrictions on the use of thioridazine in the UK. There was also a trend towards sudden death being associated with the use of high-dose antipsychotic medication. Windfuhr et al. (2011) found 286 sudden deaths in SMI over a 7-year period in England and Wales. The medication regimens most strongly associated with sudden death in that sample were clozapine, droperidol and promazine, as well as a combination of antipsychotic medications, which tends to be a proxy for high dose. Not every study confirms this picture: Manu et al. (2011) found that unexpected sudden deaths in people with schizophrenia were most 
likely to be due to coronary events and found no increased mortality related to higher levels of antipsychotic treatment. More recently, a meta-analysis of cohort and case-control studies investigating antipsychotic medication and sudden death has been reported (Salvo et al., 2016). The odds ratios (ORs) for sudden deaths compared with non-users (matched for psychiatric and cardiovascular morbidity) were quetiapine 1.72, olanzapine 2.04, haloperidol 2.97, risperidone 3.04, clozapine 3.67 and thioridazine 4.58 . However, it is noteworthy that all the studies included in this meta-analysis were observational and not randomised. Therefore there is the potential for confounding by indication and obesity and other potentially relevant factors that were not controlled for. The authors concluded that, despite matching for it, the prevalence of CVD in the different groups may underlie these findings. Some $43 \%$ of the variance was explained by the hERG blockade potency of the varying drugs, suggesting again that while delayed repolarisation is an important factor associated with these deaths, it is not the only one.

A similar finding was reported from two large cohort studies in the USA which found that the risks of ventricular arrhythmias and sudden death of patients on antipsychotic medication correlated poorly with the QT effects of the drugs and concluded that QT prolongation was a poor surrogate for antipsychotic arrhythmogenicity (Leonard et al., 2013). This study and those of Straus et al. (2004) and Reilly et al. (2002) showed that the risks of sudden death may be dose-related. Further research on this topic is needed, but it seems clear that some antipsychotic agents carry a greater risk of worsening CVD mortality than others, particularly in higher dosage.

\section{Antipsychotic medication and venous thromboembolism}

Another potential link between antipsychotic medication and cardiovascular morbidity has been identified in studies which show an elevated risk of venous thromboembolism (VTE) with antipsychotic drug prescription. Jönsson and colleagues carried out a critical review of the incidence, mechanisms and management of VTE in users of antipsychotics (Jönsson et al., 2012). This revealed that the highest risk was with clozapine, olanzapine and low-potency FGAs and the risk appeared to be correlated with dose. Elderly people did not show an increased risk of VTE to the same extent as younger subjects. The underlying biological mechanisms explaining the association between antipsychotic medication and VTE are to a large extent unknown. Several hypotheses have been proposed including body weight gain, sedation, and enhanced platelet aggregation, but these investigators were unable to find conclusive support for any specific factor and concluded that the risk of VTE in schizophrenia may also be related to the underlying disease rather than the medication. No specific management for this risk has any supportive evidence: vigilance and attention to general health are all that can be recommended at present.

\section{Clinical strategies to reduce cardiovascular risk}

The evidence regarding clinical strategies to reduce the cardiovascular risk as well as morbidity and mortality secondary to
CVD in schizophrenia is patchy and observational for the most part, and similarly so for managing CVD risk in people with schizophrenia treated with antipsychotic medication. Ringen and colleagues (2014) reviewed interventional studies in schizophrenia and reported on the benefits of smoking cessation, exercise, interventions for obesity (diet, medication and surgery) and monitoring of physical health on short-term outcomes, but no studies are available which show their benefits in terms of CVD morbidity and mortality. Shulman and colleagues (2014) also reviewed the field and drew attention to the need for baseline ECG and ECG monitoring in some circumstances, the minimisation of risk by careful choices of agents and dosage, and the need for assiduous attention to the metabolic health of patients. The uptake of statins in schizophrenia is lower than recommended in various guidelines for managing CVD. Studies investigating whether statins may increase the risk of diabetes or worsen cognition in psychosis have proved to be negative (Hanssens et al., 2007; Richardson et al., 2013) and so statins can be considered for people with schizophrenia, with similar indications to that of the general population (Blackburn et al., 2017; Cooper et al., 2016). Since antipsychotic treatment may affect liver enzymes (CK and ALT) it is advisable to check these before initiating therapy with statins.

There are multiple case reports of myocarditis within the first month of clozapine administration, independent of dose, which presents with tachycardia, shortness of breath, cough, and fatigue symptoms. The recommended monitoring protocols for myocarditis and cardiomyopathy vary widely, with some including ECG and investigations such as C-reactive protein and troponin (Citrome et al., 2016; Knoph et al., 2018; Nielsen et al., 2016). Between $12.5 \%$ and $24 \%$ of cases of myocarditis may have a fatal outcome, so early discontinuation of the medication and liaison with cardiology are recommended in this situation (Emul and Kalelioglu, 2015; Knoph et al., 2018).

\section{Recommendations regarding antipsychotic medication, cardiovascular disease and mortality}

- All patients should have a personal and family cardiovascular history taken. (D)

- Attention should be paid to identifying the risk factors for $\mathrm{CVD} /$ sudden death in psychiatric patients (increased body mass index, smoking, diabetes, raised heart rate, blood pressure and cholesterol, liver disease, poor nutrition) and modifying them where possible. Many of these factors are also exacerbated by antipsychotic medication. (D)

- Treatment with statins should be considered more frequently. ALT and CK levels should be checked prior to the initiation of such therapy. (D)

- Avoid high doses/polypharmacy (Lambiase et al., 2019) in all patients but especially in those with multiple cardiovascular risk factors, unless clinically justified. (D)

- Vigilance for cardiovascular events and cardiovascular complications of therapy is recommended in all patients. (D)

- The investigation of patients with syncope is always warranted. (D)

- Particular caution (including more detailed cardiac assessment) when prescribing antipsychotic medication is recommended if any of the following risk factors are present: 
- There is a history of cardiac disease especially ventricular arryhthmias, heart block or bradycardia or family history of long QTc/sudden death

- The patient is elderly and/or malnourished and/or has a history of alcohol use disorder

- The patient has liver, renal or electrolyte disturbance

- If precribed other medications which interfere with hepatic enzymes or prolong QTc. (D)

\section{When is an ECG recommended?}

- Indications for ECG prior to therapy:

- First-episode psychosis

- Past or family history suggestive of long QT syndrome

- Significant history of cardiac disease/arrhythmia

- Presence of cardiovascular risk factors

- Prior to a trial of high-risk, high-dose or acute parenteral antipsychotic medication

- There are other factors present which predispose to arrhythmias such as electrolyte abnormalities, central nervous system disorders, systemic disease or other drugs are prescribed which may prolong the QT interval or predispose to arrhythmias. (D)

- Indications for ECG during therapy:

- Abnormalities found on baseline ECG, for example prolonged QTc interval (greater than $440 \mathrm{~ms}$ for men and $470 \mathrm{~ms}$ for women), bundle branch block, abnormal $\mathrm{T}$ or $\mathrm{U}$ waves or frequent ventricular ectopic beats

- Multiple long QT risk factors are present

- New onset symptoms suggestive of arrhythmia (such as syncope) or cardiovascular disease occur

$\circ$ When a trial of high-dose or combined antipsychotics is undertaken

- When electrolyte abnormalities have been found

- If myocarditis is suspected in a patient prescribed clozapine. There should usually also be prompt discontinuation of this medication and referral to a cardiologist. (D)

\section{Area of uncertainty and future research}

More research is required on shared pathophysiological mechanisms between CVD and psychosis and on the mechanisms that link antipsychotic medication to adverse CVD outcomes. Future research should consider unknown mechanisms of excess mortality in schizophrenia (such as a direct effect of the illness or its treatment, the effects of mental illness on lifestyle and environment, the effect of mental illness on access to physical health care, etc.), and the impact on mortality of primary prevention approaches addressing modifiable risk factors for physical illness (Lawrence et al., 2010).

\section{Metabolic adverse effects of antipsychotic medication}

\section{Weight gain and the metabolic syndrome}

The metabolic syndrome is indicated by the presence of three or more of the following cardiometabolic risk factors: central obesity, hypertension, raised fasting glucose, and dyslipidaemia (low
HDL cholesterol and raised triglyceride levels). Meta-analysis of 126 studies, from a wide variety of geographical regions, suggests an overall prevalence of almost a third (32.5\%) among patients with psychosis (Mitchell et al., 2013), while a UK study of five NHS mental health Trusts found a prevalence of $57 \%$ in patients with established psychosis (Gardner-Sood et al., 2015). A common consequence of metabolic syndrome is the development of type 2 diabetes, the prevalence of which is increased two-fold in patients with schizophrenia, with more frequent and severe consequences than in the general population (Holt and Mitchell, 2015; Vancampfort et al., 2013). Other factors associated with an increased risk of CVD include tobacco smoking, alcohol use, poor diet, sedentary lifestyle, social deprivation and sometimes inferior medical care (see Cooper et al., 2016). While the metabolic adverse effects of antipsychotic medication may add to these other risk factors for CVD, it should also be noted (as discussed in the Mortality in schizophrenia section above) that overall mortality is lower for patients on continuing standard doses of antipsychotic medication than for those not taking any such medication (Taipale et al., 2018b; Tiihonen et al., 2009).

It is also worth noting that metabolic pathology may be present at, or prior to, the onset of schizophrenia. Two meta-analyses provide evidence for impaired glucose tolerance and insulin resistance in those with a first episode of schizophrenia (Perry et al., 2016; Pillinger et al., 2017), and there is also evidence that relatives of patients with schizophrenia have a higher than expected prevalence of diabetes (Mothi et al., 2015). However, analysis of data derived from genome-wide association studies has not provided consistent evidence regarding a possible genetic contribution to the risk of type-2 diabetes in individuals with schizophrenia (Padmanabhan et al., 2016; Hackinger et al., 2018).

Excessive weight gain is an important driver for the development of metabolic syndrome. There are a number of mechanisms by which antipsychotic medication may increase body weight, particularly blockade at D2/3, 5HT2c and histamine 1 receptors (Karr et al., 2019; Reynolds and McGowan, 2017). There is also evidence that antipsychotic medication may disrupt hormones involved in the control of food intake, such as leptin and adiponectin, and evidence that antagonism of muscarinic M3 receptors on pancreatic $\beta$-cells may contribute to glucose dysregulation (see Reynolds and McGowan, 2017). Large, multiple-treatments, network meta-analyses of clinical trials of antipsychotic medications have shown that most can lead to significant weight gain (Huhn et al., 2019; Leucht et al., 2013). From these data it seems reasonable to suggest a hierarchy of oral antipsychotic medications with respect to liability for increase in body weight, as follows (examples of medications in each group in alphabetical order): relatively low risk (amisulpride, aripiprazole, asenapine, brexpiprazole, cariprazine, haloperidol, lurasidone and ziprasidone); medium risk (paliperidone, quetiapine and risperidone); and relatively high risk (clozapine and olanzapine). This risk profile reflects the degree by which medications block 5HT2c, histamine 1 and other receptors in addition to D2/3 receptors at clinical doses (Karr et al., 2019; Reynolds and McGowan, 2017). Data from a previous, more limited meta-analysis of direct comparison RCTs (Rummel-Kluge et al., 2010) showed a similar pattern and found a similar order for the magnitude of changes in plasma glucose and cholesterol.

Significant weight gain, and increases in other metabolic indices, can be identified within 6-8 weeks of commencing antipsychotic treatment (Correll et al., 2014; Foley and Morley, 2011) 
and may average $3.8 \mathrm{~kg}$ by 12 weeks (Tarricone et al., 2010). Early weight gain appears to be a strong predictor of longer-term weight gain (Kinon et al., 2005; Vandenberghe et al., 2015). In the EUFEST study of individuals experiencing their first episode of schizophrenia, weight gain of $\geqslant 7 \%$ of initial body weight was found in nearly two-thirds (65\%) of the follow-up population at 1 year (Kahn et al., 2008). Antipsychotic-naïve patients are more vulnerable to weight gain; in contrast, patients on long-term antipsychotic medication have usually already gained significant weight and so there is less potential for futher weight gain if their antipsychotic medication were to be switched.

Prescribing decisions and discussions with the patient should take into account the potential for weight gain and development of the metabolic syndrome. It is also important to ensure continued monitoring of patients for aspects of the metabolic syndrome and cardiovascular risk. This is explicitly recommended in evidence-based guidelines (Cooper et al, 2016; NICE CG178, 2014a), although UK-wide clinical audits have demonstrated that such monitoring is often limited (Barnes et al., 2015; Crawford et al., 2014). The National Clinical Audit of Psychosis in 2018 (Royal College of Psychiatrists, 2018) found that there had been some improvements in monitoring for cardiometabolic risk factors since 2013 , with $42 \%$ of patients having evidence that all five of the most important risk factors were monitored in the previous 12 months, compared with only $27 \%$ and $34 \%$ in 2011 and 2013 , respectively. There was also evidence of improvements in the frequency of offers of interventions for these risk factors. However, the situation still remains far from that advised in the relevant guidelines.

The clinical management of excessive weight gain, impaired glucose control, metabolic problems and cardiovascular risk has been specifically addressed in another BAP guideline (see Cooper et al., 2016). However, it should be noted that while this BAP guideline recommends (in agreement with NICE CG178) that lifestyle interventions should be considered for all patients, three subsequent studies (one each in Denmark, USA and UK) have failed to find a significant benefit with such interventions for patients with psychotic disorders (Druss et al., 2017; Holt et al., 2019; Speyer et al., 2016).

\section{Management of plasma prolactin elevation}

Plasma prolactin elevation is linked to D2/3 blockade in the pituitary gland (Kapur et al., 2000). The risk of plasma prolactin elevation is greatest with antipsychotic medications such as risperidone, paliperidone, amisulpride, sulpiride and haloperidol and seems to be lowest with medications such as aripiprazole, clozapine, brexpiprazole, cariprazine and quetiapine (Haddad and Wieck 2004; Leucht et al., 2013; Taylor et al., 2018). Plasma prolactin elevation is asymptomatic in some patients, while others may exhibit side effects related to the direct effects of prolactin on body tissues (such as galactorrhoea) and secondary side effects including endocrine-related sexual and reproductive dysfunction (Byerly et al., 2006; De Hert et al., 2014). The relationships between antipsychotic-induced hyperprolactinaemia and osteoporosis and breast cancer respectively have been suggested, but the evidence as yet remains inconclusive (De Hert et al., 2016a, 2016b; Froes Brandao et al., 2016; Kishimoto et al., 2012). A switch to a prolactin-sparing antipsychotic is commonly considered as first choice but should be undertaken cautiously
(Buchanan et al., 2010; Henderson and Doraiswamy, 2008; Kelly et al., 2013), as the risks and benefits of such a change for an individual patient are difficult to predict, partly because the new antipsychotic medication may have other, unpredicted disadvantages and side effects, and partly because other factors, including genetic susceptibility and the schizophrenia illness itself, may contribute to raised prolactin (Aston et al., 2010; Houston et al., 2010). Alternatively, a meta-analysis of five RCTs found that adding aripiprazole to existing treatment (risperidone or haloperidol) was superior to placebo in normalising elevated prolactin and that a $5 \mathrm{mg}$ daily dose was sufficient and did not statistically significantly add to the side-effect burden ( $\mathrm{Li}$ et al., 2013). This effect is thought to be due to partial agonism by aripiprazole at D2/3 receptors in the pituitary, which reduces the risk of high levels of D2/3 blockade associated with hyperprolactinaemia (Kapur et al., 2000; Natesan et al., 2006).

\section{Extrapyramidal side effects}

All drugs which attenuate central dopaminergic function, directly or indirectly, contain within their pharmacology the potential to cause extrapyramidal neurological dysfunction. Because of their direct, and usually antagonistic, actions, antipsychotic medications are among the most prone. EPS comprised a prominent field of psychiatric research in the past, with quality studies providing credible data on prevalence, incidence and risk factors (Owens, 2014). With the arrival of SGAs in the 1990s, however, interest faded and no comparable body of independently funded, focussed research was maintained in relation to the newer antipsychotic medications. Nonetheless, increasing evidence covering longer periods of exposure and pragmatic, as opposed to ideal practice, suggests that the rates of major EPS disorders remain high (Mentzel et al., 2017) and in some instances do not substantially differ overall with use of newer, as compared with older, compounds (Carbon et al., 2017). Problems in interpreting the EPS research literature include differences in methodology between studies and a tendency to compare categories of antipsychotic medication (i.e. FGAs vs. SGAs), which obscures the differences in liability for EPS between individual medications (Haddad and Sharma, 2007). EPS remain a major issue with the use of antipsychotic medication: drug-induced parkinsonism and akathisia are linked to D2/3 receptor blockade in the striatum (Kapur et al., 2000), and as all current antipsychotic medications block $\mathrm{D} 2 / 3$ receptors to some degree, there is a risk of these side effects with any of these agents, particularly at high doses.

EPS are conventionally classified syndromally (acute dystonias, drug-induced parkinsonism, akathisia and tardive dyskinesia) which has clinical utility, though more than one disorder may develop in any individual patient. Practice variables, covering choice of drug (receptor binding profile, potency) and how it is used (starting doses, escalation rates, treatment and maintenance regimens), are major factors in EPS emergence (Owens, 2014) that raise prescribing issues for individual practitioners. Assessment of antipsychotic-induced movement disorders has conventionally been haphazard, but regular monitoring using standardised recording instruments should be a routine, qualityof-care issue.

With the possible exception of drug-induced parkinsonism, the pathophysiology underlying EPS is poorly or not understood and treatment is unsatisfactory. Avoidance through primary 
prevention (limiting indications, minimising exposure, avoiding risk scenarios) should be the major management focus for all disorders.

\section{Acute dystonia}

Only two, older studies have formally assessed the incidence of acute dystonias with oral antipsychotic medication, both utilising high doses of the high-potency antipsychotic, fluphenazine (Singh et al., 1990; Chakos et al., 1992). Clinical experience strongly suggests that incidence has declined substantially from the $34-36 \%$ reported then, which can be explained by lower equivalent dose utilisation, the generally lower potency and broader binding profiles of most newer antipsychotic medications, and widely implemented, gradual escalation protocols on commencement. This is supported by a meta-analysis that showed that intramuscular SGAs (aripiprazole olanzapine, ziprasidone) had a significantly lower risk of acute dystonia compared with intramuscular haloperidol alone (Satterthwaite et al., 2008).

Antimuscarinics (orally, IM with subjective symptoms only or IV depending on the situation) remain the treatment of choice (Burkhard, 2014) and are to be preferred to alternatives, such as diphenhydramine which, although possessing antimuscarinic actions, has been implicated in causation, and benzodiazepines which primarily target peripheral muscle tone (Owens, 2014).

\section{Drug-induced parkinsonism}

Drug-induced parkinsonism (DIP) is the second commonest form of parkinsonism after idiopathic Parkinson's disease (LopezSendon et al., 2012; Shin and Chung, 2012), with antipsychotic medication a major cause. Prevalence studies are, however, confounded by multiple variables independent of drug treatment, including sample composition (gender/age/age range), geography (reflecting international prescribing differences), how the disorder is defined and assessed, and when, during exposure, assessment takes place: the disorder may plateau or resolve with time. The psychiatric literature reports an overall prevalence of $15-31 \%$ in exposed samples (Owens, 2014), though the data suggest an adjusted 12-month event rate of approximately $40 \%$ (Miller et al., 2008). This relates to syndromal disorder and it is likely that with sensitive assessment, focusing on specific features, prevalence figures would be revealed as much higher.

Clinical features are extensive and dominated by manifestations of bradykinesia, which can be easily confused with mental state phenomena (such as fatiguability, dysphoria and apathy). The most important non-drug-related predisposition is a positive correlation with age. While most studies report higher rates in females, confounding with other time variables (age/age at first exposure/duration of exposure) makes it difficult to confirm gender as an independent risk. Pragmatic studies have consistently failed to show any advantage in liability for DIP for SGAs compared with FGAs (Jones et al., 2006; Kahn et al., 2008; Lieberman et al., 2005). While clear genetic predispositions have been linked to a number of presentations of Parkinson's disease, no specific mutations can yet be put forward to explain predisposition to drug-induced disorder (Owens, 2014). Traditionally, DIP has been considered reversible with cessation of the causative agent and while this is the case in the majority, reversibility cannot be assumed, especially in older patients (Stephens and Williamson, 1984). This may reflect the role of antidopaminergic medication in 'revealing' idiopathic disease (Tinazzi et al., 2014), though recent evidence also raises the possibility that DIP may predispose to later idiopathic disorder (Erro et al., 2015; FoubertSamier et al., 2012).

When identified, treatment regimens should be reviewed with the aim of reducing antipsychotic exposure, especially to highdose/high-potency, narrow-spectrum compounds. Antimuscarinics are effective in relieving symptom burden, though their impact on signs is less clear and they can impair aspects of memory and learning, especially in the elderly. In view of concern about an increased dementia risk with chronic use (Chuang et al., 2017; Gray et al., 2015; Richardson et al., 2018), antimuscarinic exposure should be minimised, kept under review and, where possible, time-limited. Dopaminomimetic drugs that form the basis of treatment for Parkinson's disease are generally avoided because of the negative inferences of the dopamine hypothesis of schizophrenia, though the clinical evidence of negative mental state outcomes is limited (Owens, 2014).

\section{Akathisia}

Akathisia (literally, 'inability to sit (still)' and tasikinesia, 'inability to stand (still)') refers to a subjective experience of unpleasant inner restlessness combined with an objective element of intense or 'driven' movement in the whole body or, more rarely, an affected part. The motor activity is not, however, involuntary, but an attempt, usually unsuccessful, to ease distress (Barnes and Braude, 1985; Braude et al., 1983). Akathisia is thus more accurately considered a neurobehavioural disorder (Owens, 2014). It is, however, a complex construct with as many as nine different subtypes described (Lang, 1994). In addition to acute disorder, chronic and tardive variations are described, though the relationship between tardive akathisia and tardive dyskinesia remains controversial (Barnes, 1987).

Much confusion of concepts and terminology exists in the literature between 'akathisia' and 'restless legs (Ekbom) syndrome', and the precise relationship between the two remains to be clarified. However, in view of many years of psychiatric research into 'akathisia', it is recommended that this concept be respected in a drug context unless there is marked atypicality of presentation, including exclusive lower limb localisation, predominant sensory dysaesthesiae, marked sleep disruption from involuntary lower limb activity or striking diurnal variability (Owens, 2014).

The prevalence and incidence of akathisia vary widely, not least because of differing diagnostic thresholds. Pragmatically derived prospective data in the older literature, combining subjective and objective components, found a 7-day incidence of $23 \%$ and overall 2 -week incidence of $31 \%$ (Sachdev and Kruk, 1994). No comparable data are available for the newer medications but lower figures are suggested: 10-16\% (amisulpride, olanzapine, quetiapine: Kahn et al., 2008); 9\% overall (Mentzel et al., 2017); and 10-12\% (Owens, 2014, narrative review). With newer, high-potency medications, rates appear similar to those reported earlier (Owens, 2014).

Unlike other EPS, pharmacological variables are the only factors clearly identified as representing predisposition to akathisia. The widely practised addition of antimuscarinics as treatment for 
akathisia has only weak evidential support except, perhaps, where akathisia emerges alongside, or in the context of, DIP (Pringsheim et al., 2018). Other interventions with some or equivocal support include beta-blockers (propranolol), clonidine, gabapentin, mianserin, trazodone and low-dose mirtazapine (Owens, 2014). Treatment outcomes for akathisia are readily confounded by non-specific drug actions, such as euphoria (from antimuscarinics) and sedation, an important consideration in evaluating the risk:benefit of additional treatments.

\section{Tardive dyskinesia}

Tardive dyskinesia (TD) is a generic term for any involuntary movement type, except tremor, believed to be caused by exposure to antidopaminergic medication, especially antipsychotics (Owens, 2014). It has long been of major psychiatric concern in view of its disfiguring and functionally incapacitating consequences and potential irreversibility (Kane et al., 1992).

Good quality, independent studies conducted in the 1980s and 1990 s reported a prevalence of $20 \%$ in younger populations with schizophrenia (Muscettola et al., 1993; Woerner et al., 1991), though this figure is inflated substantially $(>50 \%)$ with the inclusion of mild abnormality (Owens, 2019b). An annual incidence of 5\% was found (Chakos et al., 1996; Morgenstern and Glazer, 1993), which increases by some five to sixfold when first exposure is after middle life (Jeste et al., 1995). Risk does not appear to be linear over time (Chakos et al., 1996), and even after many years of incident-free treatment never fully disappears (Glazer et al., 1993). A large meta-analysis of RCTs showed a clinically meaningful, lower annualised incidence of TD with SGAs $(2.6 \%)$ compared with FGAs, principally haloperidol $(6.5 \%)$, which was not clearly driven by dosage (Carbon et al., 2018).

As any voluntary muscles(s) can be affected, the focus and spread of disorder is highly variable. In addition, as a syndrome the balance of movement types varies considerably, adding to diagnostic complexity. Approximately $80 \%$ of cases involve oral/perioral musculature, with disorder conventionally believed to start in the tongue, progressing to jaw and lips ('bucco-masticatory-lingual', or 'BML', triad). Traditionally, these disorders were referred to as 'choreoathetoid' in type, but this is clearly inaccurate in relation to the complex, recurrent and, most importantly, co-ordinated movements that predominate (Owens, 2019a). Disorder of this type has the characteristics of 'release' conditioned behaviours and has been more accurately categorised as 'tardive stereotypy'. Perioral and peripheral (trunk and limbs) disorder is more characteristically involuntary and mainly choreiform or dystonic in type (Owens, 2014). The utility of recent re-evaluation of clinically based classification from a neurology perspective (Frei et al., 2018) remains to be established.

Major risk factors include age, antipsychotic potency, exhibiting EPS, especially akathisia, early in antipsychotic treatment, and the number of drug-free intervals, which can be as brief as a month (Branchey and Branchey, 1984; Kane et al., 1992). The risk may increase threefold with more than three interruptions of continuing treatment (van Harten et al., 1998). Evidence to date points to no, or only marginal, influence from dopaminergic and other receptor polymorphisms and genotypic variants in CYP450 isoenzymes, though studies are few, small in size and generally of poor quality (Owens, 2019a).
There is no satisfactory treatment for TD, emphasising the importance of primary prevention. Attempts to modify antipsychotic regimens may be tried, but any changes of type of medication (reducing potency) and/or dose should be undertaken very gradually, over weeks or months, to minimise the risk of withdrawal-emergent exacerbation of disorder which may not entirely resolve (Owens, 2019b).

Support for the most advocated treatments springs from small, underpowered studies (Bergman et al., 2017) that, in addition, do not separate out movement types and/or distributions which may respond differentially, or allow for confounding by non-specific (e.g. sedation) effects (Owens, 2019b). Increasing antipsychotic dose may, at least temporarily, suppress disorder but should be restricted to specialist movement disorder settings. Clozapine may be helpful (Mentzel et al., 2018), especially in disorder dominated by dystonia, but the time-frame for improvement may be long. In Europe, the VMAT2 inhibitor, tetrabenazine, has been approved for many years and should be considered in disabling disorder. It is, however, not an easy drug to use and can be associated with high rates of adverse effects (Owens, 2019b). Newer compounds which maintain the presynaptic depleting action but have enhanced tolerability (valbenazine and deutetrabenazine) are licensed in the USA (Anderson et al., 2017; Fernandez et al., 2017; Hauser et al., 2017), and are likely to become more widely available (Ricciardi et al., 2019). While some patients may improve considerably on presynaptic depleters, response variability is wide and treatment should be subject to regular review.

\section{Area of uncertainty}

Direct interventionist treatments are increasingly suggested for functionally incapacitating, crippling or life-threatening TD, but trial-derived evidence remains weak. Focal orofacial disorder, especially lingual protrusion, may be improved with injection of botulinum toxin into especially the genioglossus muscle, with benefits potentially extending beyond the target disorder, though these are likely to require repeating regularly (Stegmayer et al., 2018). However, such treatments have not been subjected to adequate trial evaluation using standardised administration protocols.

As in idiopathic dystonia, deep brain stimulation is being actively investigated in patients with tardive dystonia. This is a clinically and ethically challenging area for utilisation of goldstandard trial designs and while benefits have been reported, these appear varied and unpredictable (Gruber et al., 2018; Macerollo and Deuschl, 2018). More adequate evaluation must address heterogeneity of patient samples, both neurologically and psychiatrically, and the appropriate area for electrode placement (globus pallidus versus subthalamic nucleus) in the context of varied clinical presentations.

\section{Neuroleptic malignant syndrome (NMS)}

NMS is a rare adverse effect of any antidopaminergic drug. Potentially all antipsychotic medications, including clozapine, may cause the disorder (Murri et al., 2015; Tse et al., 2015), though it can also ensue from rapid reduction/cessation of dopamine agonist drugs used in Parkinson's disease. Recent reports suggest a prevalence of $0.02-0.03 \%$, much lower than the $1-3 \%$ reported in the 1980s (Pileggi and Cook, 2016), a decline that 
was evident before the introduction of newer compounds (Keck et al., 1991) and therefore likely to be reflective of treatment practices.

Mental state abnormalities (agitation through delirium to coma) emerge first, though these can be difficult to detect in the context of florid psychosis. Almost all cases have disordered muscle tone (sustained 'lead-pipe', as opposed to 'cogwheel', rigidity) and thermoregulation (hyperthermia: a body temperature of $38^{\circ} \mathrm{C} / 100.4^{\circ} \mathrm{F}$ or more on two separate occasions) (Gurrera et al., 2011; Strawn et al, 2007). Signs of sympathetic hyperactivation (tachycardia, diaphoresis and blood pressure changes, including lability) emerge subsequently in about half of cases. Serum creatinine phosphokinase (CPK) may be substantially elevated (more than four times the baseline value) but this can occur secondary to involuntary muscle activity (agitation/hypertonicity) or recent intramuscular injections and is best viewed as a marker of neuromuscular pathology (Tormoehlen and Rusyniak, 2018). Leucocytosis may reflect NMS-specific immune activation but must not be overlooked as a pointer to underlying infection (Levinson and Simpson, 1986).

In view of its rarity, few clinicians will have had direct clinical experience of NMS and diagnosis should be based on standardised criteria. Empirically derived diagnostic criteria and international consensus-based proposals have been published (Gurrera et al., 2011; Tse et al., 2015). Detection is aided by awareness of the sequential onset of symptoms: mental state changes, followed by rigidity, then hyperthermia and subsequently autonomic dysfunction, evident in $70 \%$ of cases (Velamoor et al., 1994), but depends on placing symptomatology in the context of recent exposure to, or increasing doses of, antidopaminergic compounds, especially antipsychotic treatment, but including anti-nausea preparations, etc. (or rapid reduction/ cessation of dopamine agonist treatment). Onset is generally early in exposure, with one in six showing initial signs within $24 \mathrm{~h}$, two-thirds within a week, and $96 \%$ within 30 days (Ware et al., 2018). The differential diagnosis is extensive (Pileggi and Cook, 2016; Strawn et al., 2007) but of particular note is serotonin syndrome, a more common and hazardous cause of a very similar clinical presentation (Tormoehlen and Rusyniak, 2018).

Clinical risk factors have proved difficult to validate but experience suggests that high introductory or rapidly escalated dosage of dopamine antagonist medications, especially of highpotency compounds, is relevant (Ware et al., 2018). This is compatible with the suggestion that the decline in the incidence in the late 1980s followed a reduction in the use of high-potency antipsychotic medications administered parenterally (Keck et al., 1991). While no age group is immune, the risk is greatest in young males $(20-25$ years of age; female to male ratio is $1: 1.47$ ) (Gurrera, 2017), which is also compatible with more aggressive management for this demographic. Exhaustion, dehydration and malnutrition are suggested predisposing factors (Ware et al., 2018).

The pathophysiology of NMS is unknown but its clinical associations strongly point to marked, and probably rapid, multi-pathway central dopamine D2 inhibition (Tormoehlen and Rusyniak, 2018). A proposed genetic liability remains unconfirmed. Whether autonomic dysfunction is merely the downstream consequence of D2 inhibition or has a particular basis in dysregulated sympathetic hyperactivity (Gurrera, 1999) is unclear.
Management of NMS. Treatment is predicated on high awareness and immediate withdrawal of the putative offending dopamine antagonist medication (or re-institution of previous dopaminomimetic regimens). While most patients will respond to this alone, the need for additional supportive measures, such as rehydration, electrolyte correction and, possibly, external cooling or maintenance of alkalosis to reduce risk of rhabdomyolysis and acute renal injury, demand early involvement of intensive care medical facilities if the clinical situation progresses.

In the absence of trial data and appropriate animal models, there is no consensus should specific therapeutic interventions be required, though expert-based algorithms (e.g. Strawn et al., 2007) are available. The most widely recommended initial intervention is a high-potency benzodiazepine, such as lorazepam (1-2 mg). While algorithms offer IM or IV administration, the latter is to be preferred as regular IM administration is likely to add to clinical anxieties by complicating the interpretation of CPK levels. While the effects are usually slight and ill-sustained, improvements in subjective distress and peripheral muscle tone may make a useful contribution to care. With more marked disorder, characterised by moderately severe rigidity but temperatures up to $40^{\circ} \mathrm{C}\left(104^{\circ} \mathrm{F}\right)$ and pulse up to $120 \mathrm{bpm}$, attempts to specifically release dopaminergic 'lock-down' are recommended, with either the dopamine agonist bromocriptine, or the antimuscarinic agent, amantadine. Conventional antimuscarinics should, however, be avoided in view of their ability to block peripheral sweat secretion and thereby enhance temperature dyscontrol. With severe and/or rapidly progressive disorder, the peripheral muscle relaxant, dantrolene, may be tried, monotherapy which has been reported to be effective in $80 \%$ of patients, with a shorter time to recovery and reduced mortality (Strawn et al., 2007). Dantrolene should not be co-administered with calcium channel blockers. Even at advanced stages, electroconvulsive therapy (ECT) has been empirically observed to be both effective and safe in NMS and is recommended in severe or refractory cases (Strawn et al., 2007).

NMS-associated mortality has declined over the years to an average of less than $10 \%$ (Ware et al., 2018). Myoglobinuria is a particularly sinister development. If antipsychotic medication were the cause, re-exposure would be possible although this should be delayed, a minimum of 5 days, ideally 2 weeks (Rosebush et al., 1989; Wells et al., 1988), and attempted with a different, less potent compound (Pileggi and Cook, 2016; Ware et al., 2018). Dose escalations should be slow. Despite such precautions, recurrence may still, rarely, occur. The importance of obtaining legally valid consent for re-exposure is paramount.

\section{Recommendations regarding extrapyramidal side effects}

- EPS remain important non-target actions of antipsychotic medication and clinicians should maintain a high level of awareness and monitor for EPS regularly, preferably using a standardised recording instrument. (D)

- Practice principles revolving around choice of medication and treatment schedules impact on EPS liabilities and individually tailored treatment plans must always aim for the minimum effective dose. (A)

- Antimuscarinic agents remain the treatments of choice for acute dystonias and drug-induced parkinsonism, but 
in the absence of satisfactory drug treatments for akathisia and tardive dyskinesia, primary prevention is paramount. (A)

- The diagnosis of neuroleptic malignant syndrome should be suspected when any two of the four key clinical features (mental status change, muscle rigidity, fever, or autonomic dysfunction) appear during treatment with antipsychotic medication. (S)

- If neuroleptic malignant syndrome is suspected, antipsychotic medication should be withheld. (S)

\section{Anti-inflammatory medications}

There is converging evidence to suggest that inflammation is involved in the pathogenesis of schizophrenia (Feigenson et al., 2014; Miller and Goldsmith, 2017). One implication is that antiinflammatory agents might be re-purposed as a therapeutic strategy for this illness. Placebo-controlled clinical trials are the gold standard for evidence of efficacy, and ideally they would need to be carried out in subgroups of patients with demonstrable immune activation, such as raised circulating cytokine concentrations, which would decrease in line with clinical improvement. However, in the many studies that have measured systemic cytokine concentrations, they have been normally distributed and rarely in the pathological range (Goldsmith et al., 2016). Further, there is no consistent evidence for active inflammatory cells such as microglia in the central nervous system in schizophrenia from several studies using PET and radioligand binding to detect them (Plaven-Sigray et al., 2018). Nevertheless, the immune dysfunction hypothesis has motivated a number of clinical trials of repurposed anti-inflammatory drugs and the findings are summarised below. Published data relating to symptomatic schizophrenia associated with autoantibody encephalitis have been excluded, given the rarity of this condition (Lennox et al., 2019).

\section{Non-steroidal anti-inflammatory drugs (NSAIDs)}

NSAIDs inhibit the pro-inflammatory enzyme cyclooxygenase (COX) (Sommer et al., 2014). A meta-analysis of eight RCTs of adjunctive celecoxib, a COX-2 inhibitor, found no difference in symptoms overall, but an improvement in total and positive symptom scores in a subgroup of three studies in first-episode patients (Zheng et al., 2017a). Effect sizes were moderate. Two studies of adjunctive aspirin (one unpublished) showed an improvement in total symptom score with a small effect size (Sommer et al., 2014).

\section{Minocycline}

The tetracycline antibiotic minocycline has anti-inflammatory properties and inhibits microglial activation (Zhang and Zhao 2014). A meta-analysis of 13 RCTs in schizophrenia concluded that adjunctive minocycline improved positive, negative and total symptom scores (Zheng et al., 2019). The largest effect size was found for negative symptoms with a small effect size for positive symptoms. Three of the five largest effect sizes occurred in three studies of unspecified design published in Chinese, and a fourth was from an Iranian group that has published a series of largely positive studies of various agents on negative symptoms in the last 10 years. The two largest trials of minocycline with over 200 patients each, one in recent-onset patients (Deakin et al., 2018) and one in established schizophrenia (Weiser et al., 2019a), found no effect on positive or negative symptoms.

\section{Omega-3 fatty acids}

Omega-3 fatty acids show anti-inflammatory activity (Chen et al., 2015). As noted in the section on The at-risk mental state above, initial findings of a decreased risk of progression to psychosis in an ultra-high risk cohort with omega-3 (Amminger et al., 2010, 2015) were not replicated (McGorry et al., 2017; Nelson et al., 2018). A recent meta-analysis of 20 RCTs reported no improvement in positive or negative symptoms in schizophrenia (Cho et al., 2019b).

\section{Oestrogens}

Oestrogens decrease inflammatory mediators such as TNF- $\alpha$ (Sommer et al., 2014). Meta-analyses of double-blind RCTs of adjunctive treatment with oestrogen or the selective oestrogen receptor modulator raloxifene (Heringa et al., 2015; de Boer et al., 2018) in women have reported an improvement in positive, negative and total symptom scores with a small to moderate effect size. These findings were replicated by an RCT of the adjunctive use of an oestradiol patch in premenopausal women, but only in a subset of 100 women aged over 38 years (Weiser et al., 2019b). Whether positive results relate to anti-inflammatory or endocrine effects has yet to be established.

\section{$\mathrm{N}$-acetylcysteine}

$\mathrm{N}$-acetylcysteine (NAC) has anti-oxidant actions and is used to lessen liver damage in treating paracetamol overdoses. Oxidative stress is a feature of inflammation and NAC also inhibits inflammatory cytokines including TNF- $\alpha$ (Sommer et al., 2014). Studies have reported an improvement in negative and total symptom scores in recent-onset illness (Breier et al., 2018) and established schizophrenia (Berk et al., 2008; Farokhnia et al., 2013). One study also found an improvement in positive symptom scores in established illness (Sepehrmanesh et al., 2018). Any perceived benefit with NAC cannot be attributed to antiinflammatory actions without biomarker confirmation because it affects glutamate neurotransmission through promoting synaptic cysteine-glutamate exchange.

\section{Summary}

The trials reviewed above are generally small and of sub-optimal quality, with a lack of discontinuation studies or the use of biomarkers for inflammation to find responsive subgroups or to demonstrate target engagement. Small to moderate effect sizes often translate into a negligible clinical benefit. Larger-scale efficacy studies are needed. Given the known side-effect profile of compounds such as NSAIDs and oestrogens, there is no robust evidence for a positive benefit-to-risk ratio for their use in schizophrenia. Nevertheless interest continues, with trials in progress 
using immune suppressants such as prednisolone and monoclonal antibodies together with efforts to identify an inflammatory subgroup of psychosis that might be more sensitive to immune modulators.

\section{Recommendation regarding anti-inflammatory medications}

- The evidence is insufficient to recommend the use of antiinflammatory medications for schizophrenia in clinical practice. (A)

\section{The pharmacological treatment of schizophrenia during pregnancy and the perinatal period}

\section{Antipsychotic medication and pregnancy}

Pregnancy does not protect women from a first episode or recurrences of a schizophrenia-like illnesses: the risk of acute psychotic relapse is significantly increased in the first 2 months after childbirth (Munk-Olsen et al., 2009), and poor mental health during pregnancy is a strong predictor of mental illness in the postnatal period (McAllister-Williams et al., 2017). Since ethical constraints preclude RCTs of psychotropic medication in pregnant women, clinical practice is informed by published case reports, case series and increasingly by large database studies (Huybrechts et al., 2016; Petersen et al., 2016; Vigod et al., 2015). The former two sources of data are biased towards adverse outcomes, while database studies assume that all medicines that are prescribed are taken, and can only control for confounding variables that are documented during routine clinical care; high levels of medication non-adherence may therefore lead to any adverse effect of medication being missed while failure to capture important clinical risk factors such as smoking and obesity may result in poor pregnancy and child outcomes being wrongly attributed to prescribed medication.

Major congenital malformations (MCMs), defined as structural abnormalities of surgical, medical, functional or cosmetic importance (Tomson and Battino, 2012) occur in 2-4\% of all neonates (Nelson and Holmes, 1989). In a meta-analysis of (predominantly) prospective cohort studies, Altshuler et al. (1996) found a small, but significant excess of MCMs in infants exposed to phenothiazine antipsychotic medications as a group (exposed infants: $n=2591$; non-exposed infants: $n=71,746$; OR: 1.21 , $95 \%$ CI, 1.01-1.45). No clustering of anomalies was found, suggesting that factors other than exposure to antipsychotic medication may have been relevant. It should be noted that these early cohorts included large proportions of women who were treated for hyperemesis gravidarum with low doses of antipsychotic medication. A subsequent prospective study (Habermann et al., 2013) compared obstetric outcomes in 284 women exposed to an FGA (mostly haloperidol, promethazine or flupentixol) during pregnancy, 561 women exposed to an SGA (mostly olanzapine, quetiapine, clozapine, risperidone or aripiprazole) and 1122 women exposed to medicines known not to be harmful to the foetus. In this study, both FGA and SGA exposure were associated with numerically increased rates of MCMs; there was no clustering in the FGA group, but a modestly increased risk of cardiac septal defects in the SGA group. However, it has become increasingly clear that when compared with women who do not have schizophrenia, women who have this diagnosis have poorer physical health and social circumstances pre-conception (Galbally et al., 2019; Taylor et al., 2015; Vigod et al., 2014) that place them at a high risk of a wide range of adverse pregnancy and child outcomes, including congenital anomalies.

In the largest database study to date, outcomes from 10,000 antipsychotic-exposed pregnancies were compared with pregnancies that had not been exposed to these medicines using highdimensional propensity-matching of confounding factors, although the authors noted that there was incomplete control for maternal smoking, obesity, alcohol and other drug use (Huybrechts et al., 2016). They analysed the effects of FGAs and SGAs separately and were for the first time able to look at sufficiently large numbers of individual SGAs, namely aripiprazole, olanzapine, quetiapine and ziprasidone. In the adjusted analyses there was no association between exposure to FGAs or SGAs and either MCMs in general or cardiac defects specifically. However, there was a risk of MCMs associated with risperidone (1566 exposed pregnancies; RR 1.26; 1.02-1.56). The relationship with cardiac defects became significant at a dose of $2 \mathrm{mg}$ or more (RR, $2.08 ; 1.32-3.28)$. Although currently an unreplicated finding, the large number of exposed pregnancies in this study supports clinical caution with risperidone until further data are available.

When poorer pre-conception health was controlled for in a high-dimensional, propensity-matched, population-based cohort study of obstetric and neonatal outcomes that compared 1021 women who were exposed to antipsychotic medication during pregnancy with 1021 women who were not, no important differences were found (Vigod et al., 2015); these included gestational diabetes, hypertensive disorders of pregnancy, VTE, pre-term birth and having a baby that was small or large for gestational age. However, the authors noted that 'the rate of adverse outcomes is high enough to warrant careful assessment of maternal and foetal well-being among women prescribed an antipsychotic drug in pregnancy'. Consistent with these conclusions, a subsequent large UK cohort study based on primary health care records identified increased caesarean section rates, poor birth outcomes and neonatal neurodevelopmental problems in women exposed to antipsychotic medication during pregnancy, although these adverse outcomes were no longer significant when maternal age, obesity, smoking, alcohol use, diabetes and hypertension were controlled for (Petersen et al., 2016).

In summary, the available data are supportive of antipsychotic medications not being associated with clinically significant risks during pregnancy, but a lack of certainty about the timing of exposure and medication adherence do not allow the exclusion of the possibility that some antipsychotics may be associated with an increased risk of relatively uncommon poor obstetric outcomes or MCMs.

There is a remarkable dearth of systematic studies of neonatal reactions to late pregnancy exposure to antipsychotic medication. Several cases of neonatal EPS have been reported in response to FGAs (Gentile, 2010) but systematic motor examinations of newborns are not available. In the only controlled study that looked for drug withdrawal symptoms, there was no association with prebirth exposure to monotherapy with FGAs or SGAs (Habermann et al., 2013). There are also limited data regarding potential neurobehavioural sequelae of foetal exposure to FGAs. 
Follow-up studies have not shown differences in behavioural functioning or IQ up to 5 years, compared with unexposed controls (Altshuler et al., 1996; Thiels, 1987). In one small controlled prospective study covering the first 12 months of life after in utero exposure to either clozapine, risperidone, sulpiride, olanzapine or quetiapine, an initial worse performance on most scores of the Bayley scales resolved by the end of the first postnatal year (Peng et al., 2013).

Compared with pregnancy, much smaller amounts of psychotropic drugs are transferred to the baby during breastfeeding, although there are exceptions. A measure of exposure that is frequently used in paediatric practice is the relative infant dose (RID). This is defined as the daily amount of drug ingested by an infant, who is fully breastfed, per kg bodyweight divided by the maternal daily dose per $\mathrm{kg}$ body weight. A value of more than $10 \%$ is regarded as generally indicating a higher probability of side effects in the infant (Bennett, 1996). In respect of antipsychotic medications, small case numbers to date suggest very low transfer into breastmilk except for amisulpiride, sulpriride, risperidone and haloperidol, where estimated RIDs can approach or exceed 10\% (Hale, 2012; Toxicology Data Network, 2019).

Among the small number of published clinical observations of breastfed infants relatively few adverse effects have been described. Sedation, a slowing of development, and irritability have been noted in some cases, especially when another antipsychotic or sedative medication was co-prescribed (Toxicology Data Network, 2019). Aripiprazole can lower postpartum prolactin levels and this appears to have caused lactation failure in some cases (Toxicology Data Network, 2019). Clozapine is associated with a risk of neonatal seizures and blood dyscrasias (Parikh et al., 2014; Uguz, 2016).

In a recent study, Westin et al. (2018) reported on plasma levels of quetiapine, olanzapine and aripiprazole in the perinatal period. They found an increasing decline in plasma levels as pregnancy progressed for quetiapine and aripiprazole but not olanzapine. In the third trimester, quetiapine levels were as much as $76 \%$ and aripiprazole levels $52 \%$ lower than in the preconception and postnatal period. These results are consistent with what is known about the pregnancy-induced changes in cytochrome P450 enzyme activity (Westin et al., 2018).

\section{Selective serotonin reuptake inhibitor (SSRI) antidepressants and pregnancy}

In addition to antipsychotic medication, women with schizophrenia are often prescribed SSRIs (to manage persistent negative symptoms and/or comorbid depression) and valproate (to manage mood instability and aggression/impulsivity). A brief account of the use of these medicines in pregnancy is given below. For a more detailed account of the evidence base, readers are referred to the BAP consensus guidance on the use of psychotropic medication preconception, in pregnancy and postpartum (McAllisterWilliams et al., 2017).

With respect to SSRIs, database studies that have not controlled for maternal depression have reported an increased risk of cardiac septal defects in neonates exposed to SSRIs in utero (Gao et al., 2018; Kallen and Otterblad, 2007; Shen et al., 2017), but for the majority of SSRIs this effect is no longer statistically significant when maternal depression (Gao et al., 2018; Huybrechts et al., 2014) and lifestyle risk factors are controlled for
(Huybrechts et al., 2014); data relating to sertraline are insufficient to allow an effect to be excluded (Gao et al., 2018).

Antidepressant use in pregnancy is associated with a modest reduction in gestation (3 days) and in Apgar score (0.37 at $1 \mathrm{~min}$ ), which although statistically significant are unlikely to be clinically significant (Ross et al., 2013). Exposure to SSRIs in the last month of pregnancy may increase the risk of postpartum haemorrhage (Heller at al., 2017) but perhaps to a lesser degree than serotonin-norepinephrine reuptake inhibitors (SNRIs), for which an absolute risk increase of 4 per 100 exposures has been reported in one study by Hanley et al. (2016). Plausible pharmacological mechanisms include reductions in serotonin-mediated uterine contraction and impairment of haemostasis (Palmsten et al., 2013). Third trimester exposure has also been associated with persistent pulmonary hypertension of the newborn (absolute risk increase of 1 per 1000 births), although confounding factors are likely to be responsible for much of this increase (Huybrechts et al., 2015). When SSRIs are taken in very late pregnancy, the neonate is more likely to have symptoms of the poor neonatal adaptation syndrome, including poor feeding, irritability, lethargy and respiratory distress; whether this is secondary to serotonin toxicity, antidepressant discontinuation symptoms or early birth is unknown (Boucher et al., 2008).

\section{Valproate and pregnancy}

There is now little doubt that exposure to valproate poses a wide range of serious risks to the intrauterine development of children. As many as $11 \%$ of exposed children are born with MCMs (Meador et al., 2008) which can be severe and affect several organ systems. Risks are increased in particular for neural tube defects, congenital heart anomalies, cleft palate, radial ray defects, and ophthalmological and genitourinary anomalies (Clayton-Smith et al., 2019). Compared with children of mothers who have untreated epilepsy or no epilepsy, exposed children are seven times more likely to have developmental delay (Veroniki et al., 2017), and five times more likely to have autism (Christensen et al., 2013). It is also thought that there are milder forms of neurodevelopmental problems affecting even more children (Clayton-Smith et al., 2019). Facial dysmorphism can accompany these problems (Clayton-Smith et al., 2019). Although relationships of adverse effects with daily dose have been described, no dose has been identified as safe and folic acid supplementation has been found to be either not or only partially effective (Ban et al., 2015). Prescribing valproate after the first trimester is not thought to be safe since its neurodevelopmental effects are thought to operate throughout pregnancy.

Previous regulatory measures to minimise exposure of unborn children to valproate have not been sufficiently successful (European Medicines Agency, 2018). This led the European Medicines Agency to recommend that valproate should not be used in pregnant girls or women with mental illness or migraine and only be prescribed to pregnant patients who suffer from a severe form of epilepsy that has not responded to other drugs; in any non-pregnant women with childbearing potential, valproate should only be prescribed when a pregnancy prevention programme is followed (European Medicines Agency, 2018). This was approved by the European Commission and all European Countries in 2018. The Medicines Health Care Products Regulatory Authority (2018) in the UK has supported the 
requirement with an ongoing extensive programme of raising awareness among clinicians and patients and has put measures in place to ensure adherence to the new regulations.

\section{Resources for clinicians and patients}

For women who are pregnant or have childbearing potential and are prescribed valproate, the MHRA has developed tools for health professionals and patients that explain the new regulations, detail the responsibilities of clinicians, and facilitate participation in the pregnancy prevention programme. This programme includes discussing the known risks of the medication on the foetus (teratogenicity) and infant with the woman, providing a copy of the Patient Guide, ensuring that arrangements are in place for highly effective contraception (intrauterine devices, progestogen implants, sterilisation, etc.) and signing (by the prescriber and patient) the Acknowledgement of Risk form; the tools may be accessed on the MHRA website. Following requests by clinicians, the Royal College of Psychiatrists published clinical guidance on alternatives to and withdrawal from valproate in the treatment of women with bipolar disorder who are pregnant or have childbearing potential (Baldwin and Wieck, 2018). Also at the request of clinicians, clinical guidance was published on behalf of the Royal College of General Practitioners, Association of British Neurologists and Royal College of Physicians (Shakespeare and Sisodyia, 2019), addressing the sometimes difficult capacity and contraceptive issues arising from the new valproate regulations, particularly in children, adolescents and patients who have intellectual disability with comorbid psychiatric disorders or epilepsy. These guidance documents are available on the Colleges' websites.

A more detailed account of the use of psychotropic medicines during pregnancy and breastfeeding can be found in the 2014 NICE guideline on antenatal and postnatal mental health (NICE, 2014b). The 2017 perinatal medication consensus guidance by the BAP (McAllister-Williams et al., 2017) is aimed at mental health professionals, discusses general principles of prescribing in this patient group, critically reviews the evidence and gives detailed clinical recommendations.

The UK Teratology Information Service (UKTIS) publishes regularly updated monographs on current evidence for the reproductive safety of medicines. A useful source of up-to-date information in lay language maintained by UKTIS can be found on the 'Best Use of Medicines in Pregnancy' website (BUMPS: http://www.medicinesinpregnancy.org/). Patients should be encouraged to create their own online 'BUMPS' record and complete this throughout their pregnancy and the early postnatal period, thus adding their own data to the evidence base.

The Toxicology Data Network (2019) (https://toxnet.nlm.nih. gov/newtoxnet/lactmed.htm) provides a regularly updated reference source of the evidence on psychotropic prescribing in breastfeeding women as well as helpful clinical recommendations.

\section{Recommendations regarding the pharmacological treatment of schizophrenia during pregnancy and the perinatal period}

\section{General principles}

- Discussions with the patient about reproductive issues should include the partner or significant carer as appropriate. (S)
- All discussions about reproductive issues should be recorded in the clinical records. (S)

- When there is uncertainty about medication management, referral of the patient to a specialist perinatal psychiatry clinic for advice is recommended. (S)

In all women with schizophrenia of childbearing potential who are taking antipsychotic medication

- Clinicians should be aware that at least $50 \%$ of all pregnancies are unplanned. (S)

- Clinicians should regularly review family planning intentions with the patient. (S)

- Clinicians should explain to women how hyperprolactinaemia during antipsychotic therapy may affect fertility.

- Clinicians should ensure that the women receive contraceptive advice from their GP or a family planning clinic. (S)

- The use of valproate in this group of women should be avoided if at all possible. When valproate is used, the prescriber must fulfil all the requirements of the mandatory MHRA pregnancy prevention programme. (B)

\section{If a woman with schizophrenia is planning a pregnancy}

- The woman's psychiatric history and her response to treatment should be carefully reviewed and the risk of discontinuing medication evaluated. Poor mental health during pregnancy is a strong predictor of mental illness in the postnatal period. (S)

- In women with schizophrenia, comorbid physical conditions can contribute to a range of adverse obstetric and neonatal outcomes. (B)

- Lifestyle advice should be offered, particularly the need to eat a balanced diet and maintain a healthy weight, to stop smoking and abstain from alcohol and other drug use. (S)

- In the 3-month periods before and after conception, a higher dose $(5 \mathrm{mg} /$ day) of folic acid supplementation should be taken by women with a higher risk of having a child with a neural tube defect or other folic acid-sensitive congenital anomaly, because of diabetes, obesity, or a diet that is lacking in folate (Denison et al., 2019; NICE 2015; Wilson et al., 2015). (C)

- Any valproate medication should be discontinued unless the patient is suffering from a severe form of epilepsy that has not responded to any other medication.

- When a woman is taking an antipsychotic medication with the potential for hyperprolactinaemia, check the serum prolactin level. Should there be uncertainty about the interpretation of prolactin levels or their impact on fertility, the opinion of an endocrinologist should be sought. (S)

- The woman should be informed that while there is no absolute certainty that antipsychotic medications are safe for the developing child, available evidence suggests that they are not major teratogens, and it does not appear that developmental delay up to the age of 5 years can be attributed to this medication. (B)

- When risk factors for type 2 diabetes mellitus are present, olanzapine should be avoided unless the patient's history 
suggests that a switch to another medication enhances her risk of recurrence significantly. In the case of clozapine, concerns about the potential for relapse usually outweigh concerns about its dysglycaemic effect. (S)

- If a woman is successfully established on antipsychotic depot/LAI medication, it should be continued if the risk of recurrence is high. (S)

- Avoid polypharmacy and use the lowest effective dose. (S)

\section{If a woman with schizophrenia is pregnant}

- Clinicians should be aware that unplanned pregnancies are often discovered when the most susceptible period for teratogenicity has passed. (C)

- On discovery of the pregnancy, abrupt discontinuation of antipsychotic and potentially other psychotropic medications should be avoided due to the increased risk of a relapse. (C)

- Where available, pregnant women with schizophrenia should be referred to the local perinatal psychiatry service. $(\mathrm{S})$

- Most women with established schizophrenia should continue the treatment that is currently keeping them well. (S)

- If a woman is not taking antipsychotic treatment and such treatment is clinically indicated, the choice of medication should follow the respective recommendations for firstepisode schizophrenia or established schizophrenia. (S)

- Depot/LAI antipsychotic medication should, if possible, not be prescribed for the first time in pregnancy because of the lack of flexibility in dosing. (S)

- Avoid polypharmacy and use the lowest effective antipsychotic dose. (S)

- Compared with the general population, women with schizophrenia are at higher risk of obstetric complications and poor neonatal outcomes. Such women should be carefully monitored during pregnancy and post-natally and should give birth in a setting with access to a special care baby unit. (S)

\section{In the neonatal period}

- Check neonatal neutrophil count after antenatal exposure to clozapine. (S)

- Neonates exposed to especially high-potency antipsychotic medication in late pregnancy should be monitored for EPS, in particular acute dystonias, for several days. (D)

\section{Antipsychotic medication during breastfeeding}

- Avoid polypharmacy and use the lowest effective antipsychotic dose. (S)

- Advise women not to breastfeed when taking clozapine. (D)

- In patients with previous illness episodes, the choice of antipsychotic medication should primarily be based on previous treatment responses. (S)

- When prescribing antipsychotic medication for the first time, agents with less sedating properties should be preferred. (S)
- Monitor the neonate for alertness and poor feeding, especially when other sedative drugs are co-prescribed. (D)

\section{Key uncertainty}

A single large recent database study (Huybrechts et al., 2016) reported an increased risk of MCMs in neonates exposed to risperidone in utero. But given that a systematic review failed to find a substantially increased risk with risperidone (Ennis and Damkier, 2015), and the absence of an apparent biological mechanism, it is currently uncertain whether this antipsychotic has teratogenic potential.

\section{Pharmacological management of negative symptoms}

\section{Nature and assessment of negative symptoms in schizophrenia}

The negative symptoms refer to aspects of a person's normal mental and physical functioning that have been diminished or lost since the onset of illness. They include limited affective responsiveness (flattended affect), poverty of speech (alogia), inability to experience pleasure (anhedonia), lack of desire to form relationships (social withdrawal), lack of motivation (avolition), and lethargy. Primary negative symptoms are associated with the underlying pathophysiology, while secondary negative symptoms are attributable to other factors such as residual positive (psychotic) symptoms causing paranoid withdrawal, drug side effects of sedation and reduced spontaneous movements (bradykinesia, akinesia), and overlap with symptoms of depression (Krynicki et al., 2018; Siris, 2000). Currently available antipsychotic medications have limited clinical effectiveness for negative symptoms and no pharmacological intervention has been approved for this indication other than amisulpride, which is licensed for schizophrenia with prominent negative symptoms, including patients characterised by predominant negative symptoms. Thus, many patients have persistent negative symptoms despite optimum management of their positive symptoms and secondary negative symptoms. Along with cognitive impairment, the negative symptoms, particularly the avolition/amotivation domain, may be the main contributors to the poor functional outcomes and quality of life for people with schizophrenia (Milev et al., 2005; Villalta-Gil et al., 2006). Their treatment has been identified as an important unmet clinical need (Kirkpatrick et al., 2006).

Many tools have been developed for measuring negative symptoms. In clinical trials, the commonly used measures are the negative symptom subscale of the PANSS, the withdrawal-retardation subscale of the Brief Psychiatric Rating Scale (BPRS-WR; Overall and Gorham, 1962), and the Scale for the Assessment of Negative Symptoms (SANS; Andreasen, 1989). Recent research suggests that negative symptoms may best be represented by two relatively independent domains: expressive deficits (reflecting diminished outward expression and speech) and avolition/amotivation (reflecting diminished motivation, pleasure, and social engagement). The Clinical Assessment Interview for Negative Symptoms (CAINS) has been developed more recently for assessing these domains with high validity and reliability (Kring 
et al., 2013), and provides some improvements over older instruments, and alongside this, the Brief Negative Symptom Scale (BNSS) has also been developed, which requires less time to complete than the CAINS, but shows similarly strong psychometric performance (Kirkpatrick et al., 2011). To date, most rating scales have been based on observer ratings. However, the Self-evaluation of Negative Symptoms (SNS) has been developed and validated for patient self-assessment (Dollfus et al., 2016).

\section{Effects of antipsychotic medication on negative symptoms}

Non-clozapine antipsychotic medications for negative symptoms. Fusar-Poli and colleagues (2015) carried out a comprehensive meta-analysis of randomised, placebo-controlled trials of a range of interventions for negative symptoms in schizophrenia, including FGAs and SGAs. Compared with placebo, SGAs reduced negative symptom scores to a statistically significant extent but the effect size did not reach the threshold set by the investigators for a 'minimally detectable clinical improvement over time'. These findings do not provide support for the notion that antipsychotic treatment is associated with clinically meaningful improvements in negative symptom burden. Indeed, as the improvement may be at least partly attributable to reductions in secondary rather than primary negative symptoms, any benefit seen in these studies may overestimate the true effects. Thus, Fusar-Poli and coleagues (2015) concluded that antipsychotics are not clinically effective treatments for negative symptoms.

This is consistent with a more recent meta-analysis (Leucht et al., 2017) of all acute-phase placebo-controlled antipsychotic drug trials in schizophrenia since the introduction of chlorpromazine in 1953 (167 RCTs, 28,102 participants), although studies of patients with predominantly negative symptoms were excluded. It found that although the effect sizes for negative symptoms were statistically significant across antipsychotic medications (with the exception of chlorpromazine), they were small. They also observed that the effect sizes in the earlier meta-analysis (Fusar-Poli et al., 2015) were likely to be overestimated due to an error in the methodology for their calculation.

Clozapine monotherapy for negative symptoms. The metaanalysis of treatments for negative symptoms in schizophrenia (Fusar-Poli et al., 2015) noted above found no placebo-controlled trials of clozapine published in the literature. In earlier systematic reviews of head-to-head comparisons of clozapine with other antipsychotics in treatment-resistant schizophrenia, short-term data from the SANS negative symptom scores favoured clozapine over FGAs (Essali et al., 2009), while in comparison to SGAs clozapine was found to be less effective for negative symptoms than quetiapine and not significantly different to olanzapine and risperidone (Asenjo Lobos et al., 2010). With regard to the finding of superiority of clozapine over FGAs, it is possible that this was due to fewer secondary rather than primary negative symptoms as there is an overlap between the bradykinesia component of parkinsonism (for which most other antipsychotic medications have a greater liability than clozapine) and both the expressive deficits and avolition-amotivation domains.

More recent meta-analyses of RCTs have differed slightly in the details but support the conclusion that effects on negative symptoms do not differ to a clinically significant extent between clozapine and other antipsychotic medications (Samara et al., 2016; Siskind et al., 2016). In one analysis (Samara et al., 2016), the effect of clozapine on negative symptom scores was marginally inferior to olanzapine and comparable with a range of other FGAs (fluphenazine, haloperidol, chlorpromazine) and SGAs (olanzapine, ziprasidone, quetiapine, risperidone, sertindole). In another (Siskind et al., 2016), clozapine was modestly superior to other FGAs (chlorpromazine, haloperidol) and SGAs (olanzapine, risperidone, quetiapine, ziprasidone) in short-term studies (less than 3 months, with a small effect size), but not in longerterm studies (over 3 months). The conclusion from the RCT literature is supported by a meta-analysis of non-randomised cohort studies (Masuda et al., 2019) which found that the effect of clozapine on negative symptoms was not significantly different from oral, non-clozapine SGAs (risperidone, olanzapine, quetiapine, aripiprazole, amisulpride). Data on clozapine for schizophrenia that is not treatment refractory are sparse. However, a randomised open-label study comparing clozapine and risperidone in treatment-naïve first-episode schizophrenia found only marginal improvement in negative symptom scores in patients on clozapine at 1 year (Sanz-Fuentenebro et al., 2013).

There is evidence that non-pharmacological treatments for negative symptoms, such as social skills training and CBT, may be effective for negative symptoms, and meta-analyses support both approaches. However, most trials of non-pharmacological treatments have had small sample sizes, lacked standardisation of assessments and did not follow up patients for sufficiently long periods. Further well-designed studies are required before recommendations can be made about using these methods as a standard treatment for negative symptoms (Galderisi et al., 2018).

\section{Pharmacological interventions}

Adjunctive antidepressant medication. In their meta-analysis of randomised, placebo-controlled trials of a range of interventions for negative symptoms in schizophrenia, Fusar-Poli and colleagues (2015) found that adding an antidepressant to antipsychotic medication was associated with a statistically significant small reduction in negative symptoms. In a large meta-analysis focusing on the efficacy and safety of antidepressants added to antipsychotic medication in schizophrenia (Helfer et al., 2016), antidepressants modestly reduced negative symptoms but such benefits were not considered likely to be clinically meaningful. Moreover, none of the studies discriminated between secondary and primary negative symptoms, and the possibility cannot be excluded that any reduction observed was at least partly due to improvement of the former. Sample sizes were too small to enable sensible judgements about the relative efficacy of individual antidepressant medications, although there was some suggestion that the effect was largest for duloxetine and mirtazapine. Nevertheless, the duloxetine data amounted to a single small study, and in their systematic review of meta-analyses of pharmacological co-treatment strategies (which included the Helfer meta-analysis of antidepressants), Correll and colleagues (2017a) pointed out that the effect sizes reported in the meta-analyses were inversely correlated with the content quality. They concluded that no pharmacological combination strategy, including adjunctive antidepressant medication, had sufficiently 
high-quality or consistent efficacy results to support recommending them over antipsychotic monotherapy.

In another meta-analysis of antidepressant augmentation strategies of antipsychotic treatment in patients with insufficient response to antipsychotic monotherapy, Galling and colleagues (2018) purposefully excluded the results from studies in which antipsychotic and antidepressant were started at the same time (co-initiation trials) in order to make the set of trials and populations more homogeneous and to better reflect common clinical practice. For negative symptoms, augmentation with an antidepressant was statistically superior to placebo, but with a small effect size and only in studies augmenting FGAs, not SGAs. The superiority was evident for SSRI (reuptake inhibitor, SERT) and SNRI (reuptake inhibitor, SERT and NET) antidepressants, but not with other antidepressant classes or individual drugs. Indeed, in contrast to other meta-analyses which have found mirtazapine effective for negative symptoms (Hecht and Landy, 2012; Helfer et al., 2016), this meta-analysis found it selectively effective only for positive symptoms. The improvement in negative symptoms did not appear to be due to a reduction in depressive symptoms, although a reduction of other secondary negative symptoms cannot be excluded. Although the clinical relevance of the small effect size found is doubtful, this could not be addressed further as studies reporting the proportion of responders (i.e. the percentage of participants reaching pre-defined thresholds of negative symptom improvement) used heterogeneous definitions of response and showed evidence of reporting bias.

Addition of a second antipsychotic. Galling and colleagues (2017) conducted a meta-analysis of RCTs focusing exclusively on the addition of a second (non-clozapine) antipsychotic after nonresponse to the first (clozapine or non-clozapine). Negative symptoms statistically significantly improved with the antipsychotic combination but with a small effect size that was even smaller when only high-quality studies were included. In subgroup analyses, this effect was only significant in studies augmenting with aripiprazole. Augmentation of clozapine was not reported separately, but was found to be non-significant in another meta-analysis (Correll et al., 2017a). Given the small effect size, clinical significance is unlikely and the augmentation of a first antipsychotic by a second, even aripiprazole, cannot be recommended for treating negative symptoms based on current evidence.

Other pharmacological strategies. Glutamatergic drugs have been reported to result in a small, statistically significant, reduction in negative symptoms. However, this falls well short of clinical significance (Fusar-Poli et al., 2015). A commercial trial of a glycine reuptake inhibitor (bitopertin) for negative symptoms failed at Phase III (Bugarski-Kirola et al., 2017).

In a systematic review of meta-analyses, Correll and colleagues reported on the efficacy of 42 pharmacological strategies that had been combined with antipsychotics for sub-optimal treatment response in schizophrenia (Correll et al., 2017a). Their conclusions on adding an antidepressant or another antipsychotic have already been mentioned above. For negative symptoms, none of the five pharmacological combinations with clozapine (antidepressant, topiramate, lamotrigine, another antipsychotic, and glycine) outperformed placebo. Of the 26 pharmacological add-on medications with non-clozapine antipsychotics, 12 were statistically significantly superior to controls. Five of these
(SSRIs, modafinil, any antidepressant, oestrogens, and another antipsychotic) had small effect sizes; two (topiramate and minocycline) had medium effect sizes; and five (testosterone, acetylcholinesterase inhibitors, lamotrigine, 5-HT3 receptor antagonists and SNRIs [a single study on duloxetine]) had large effect sizes.

The following treatment strategies were not statistically significant for negative symptoms: noradrenaline reuptake inhibitor antidepressants (NRI); lithium; NSAIDs; varenicline; azapirones; adenosine modulators;dehydroepiandrosterone (DHEA); noradrenergic and specific serotonergic antidepressant (NaSSA); monoamine oxidase-B (MAO-B) inhibitors; carbamazepine; serotonin antagonist and reuptake inhibitor (SARI) antidepressant (trazodone); oxytocin; pregnenolone; and glutamate NMDA receptor antagonism.

However, across the systematic review the quality of the meta-analysed studies was generally low, and the effect sizes reported in the meta-analyses were inversely correlated with the content quality. No pharmacological combination strategy had sufficiently high-quality or consistent efficacy results to support a recommendation over antipsychotic monotherapy for clinical care guidelines for any of the measured schizophrenia outcomes, including negative symptoms, which contrasts with the treatment recommendations that emerged from those meta-analyses. The authors of this systematic review conclude that there are no grounds for recommending any pharmacological combination treatment for schizophrenia based on the current evidence but note that further well-designed clinical trials of add-on treatments are required.

In summary, although statistically significant reductions in negative symptoms scores were found with several pharmacological strategies, the effect sizes were small and of doubtful clinical significance. Where their clinical relevance has been estimated (for antidepressants, antipsychotics, and glutamatergic drugs), the changes in negative symptom scores have fallen well short of the minimum clinical improvement detectable by clinicians (FusarPoli et al., 2015), although it remains possible that there may be a subset of patients who may respond more dramatically to these interventions. A systematic overview and quality appraisal of the range of pharmacological strategies investigated to date has concluded that no single strategy for reducing negative symptoms can be recommended for unselected patients with schizophrenia on the current meta-analytic literature (Correll et al., 2017a).

Despite the lack of evidence from unselected trials, it is possible that pharmacological treatments may benefit particular patients.

\section{Recommendation regarding the pharmacological management of negative symptoms in schizophrenia}

- If a trial of medication for negative symptoms is to be attempted, it would be advisable to use one of the newer rating scales for negative symptoms (CAINS or BNSS) to monitor treatment response, with a plan to discontinue treatment if no clinically meaningful response is seen. (D)

\section{Key uncertainties}

In the literature to date, the contribution of primary negative symptoms (if any) to the small reductions in negative symptom 
scores seen with some pharmacological interventions is uncertain. In order to minimise the contribution of secondary negative symptoms, future studies should focus on patients with stable and severe negative but minimal positive and depressive symptoms (Helfer et al., 2016; Marder et al., 2013). In addition, as two relatively independent negative symptoms domains have been identified (expressive deficits and avolition/motivation), future studies should aim to discriminate between the effects of pharmacological interventions on the two factors (Barnes et al., 2016).

Small average effect sizes in large groups may mask smaller subgroups of patients with greater benefit. The field should advance by moving from study-level to patient-level meta-analyses and the identification of subgroups of patients with the highest likelihood of response to combination strategies. In addition, there is an urgent need for qualitatively excellent and sufficiently large RCTs to counteract the reliance of treatment recommendations on meta-analyses of multiple, relatively poor quality trials (Correll et al., 2017b). The importance of large RCTs is underscored by the illustration that while a meta-analysis of nine relatively small trials of minocycline in schizophrenia (Xiang et al., 2017) reported a statistically significant superiority for minocycline, a subsequent high-quality RCT with the largest number of subjects to date (the BeneMin Study: Deakin et al., 2018) found no benefit for minocycline on any of the outcome measures, including negative symptoms. Further large high-quality RCTs are required investigating clinically relevant outcomes of augmentation with aripiprazole, as well as the more recent D2 partial agonists, brexpiprazole and cariprazine. In addition, augmentation with them will need to be compared with simply switching to them or increasing the dose of the first antipsychotic medication (Galling et al., 2017).

Future meta-analyses should aim to answer more focussed clinical questions and improve the homogeneity of study populations by selecting only studies in which the interventions are highly similar and of direct relevance to the clinical question being addressed in the meta-analysis (Galling et al., 2018). Future RCTs should also report on all possible outcomes, particularly clinically meaningful ones such as response/remission rates, CGI change scores, quality of life, and functioning. Reductions in negative symptoms which are not apparent to clinicians or not associated with any improvement in quality of life and/or functioning for the patient are unlikely to be clinically relevant, notwithstanding their statistical significance.

\section{Pharmacological strategies for cognitive impairment}

It is well established that the cognitive dysfunction in schizophrenia is generalised, affecting virtually every neuropsychological domain, with impaired information processing speed being particularly pronounced (Dickinson et al., 2007; Joyce, 2013). Neuropsychological performance in people with schizophrenia is estimated as being $0.5-1.0$ standard deviations below the general population mean and is present by the time of psychosis onset, although it does not progress further (Leeson et al., 2011). Cognitive dysfunction, which can be differentiated from negative symptoms (Harvey et al., 2006), may be more predictive of functional outcome than either negative or positive symptoms (Nuechterlein et al., 2011). Therefore, early amelioration of cognitive impairment may have the potential to improve social and occupational function over the long term.

The majority of RCTs examining cognitive function in schizophrenia can be grouped into those studying cognitive enhancers (such as acetylcholinesterase inhibitors and memantine) already shown to benefit patients with Alzheimer's disease and other dementias and those derived from promising preclinical studies addressing specific hypotheses regarding the neurobiological basis of schizophrenia (such as NMDA, nicotinic and oestrogen receptor agonists or modulators).

\section{Acetylcholinesterase inhibitors}

Santos et al. conducted a meta-analysis of nine RCTs in which donepezil, galantamine, rivastigmine or placebo were added to current antipsychotic medication in stable patients with schizophrenia (Santos et al., 2018). Of the three cognitive domains examined, speed of processing improved, attention worsened and working memory did not change. The total number of participants was relatively low (175 active drug vs. 173 placebo) and the quality of the evidence was considered moderate or low. Overall, the authors had little confidence in the estimates of effect and the evidence was too weak to allow for any recommendation regarding the use of acetylcholinesterase inhibitors in schizophrenia.

\section{Memantine}

Like the acetylcholinesterase inhibitors, memantine is approved for use in Alzheimer's disease. Its action as an uncompetitive low affinity NMDAR partial antagonist is thought to mediate improved cognitive function in dementia by blocking excess NMDA receptor activity (Bhakta et al., 2016). Regarding its potential for schizophrenia, memantine has been shown to enhance performance on tasks measuring neurophysiological markers of information processing, such as pre-pulse inhibition (PPI) and mismatch negativity $(\mathrm{MMN})$, which are known to be impaired in schizophrenia (Bhakta et al., 2016). However, acute administration of memantine at a dose shown to improved PPI and MMN did not improve performance on neuropsychological tests in patients with schizophrenia (Bhakta et al., 2016; Swerdlow et al., 2016). It is possible that chronic dosing is required to improve cognition and, in this regard, eight RCTs of adjunctive daily memantine have been subjected to two independent meta-analyses (Kishi et al., 2017b, Zheng et al., 2018). Three of these studies assessed cognition with the Mini Mental State Examination. There was a highly significant beneficial effect of memantine $(n=48$; MD: $3.09 ; 95 \% \mathrm{CI}$, -4.42 to 1.77 ; Zheng et al., 2018), indicating that RCTs with more robust and comprehensive neuropsychological testing would be worthwhile.

\section{NMDA receptor agonists and modulators}

The glutamate hypothesis of schizophrenia proposes that there is reduced NMDA glutamate receptor function. This is partly based on the observation that phencyclidine and ketamine, drugs with NMDA receptor antagonist properties, can cause schizophrenialike symptoms (Buchanan et al., 2007). Studies in animals and man have shown that NMDA receptor agonists can induce 
synaptic plasticity and improve memory (Goff, 2017), suggesting that enhancing glutamate transmission at the NMDA receptor may have cognitive enhancing effects in schizophrenia.

Several RCTs in people with schizophrenia have assessed the cognitive effect of agents that modulate neurotransmission at the NMDA receptor glycine binding site including the intrinsic coagonists glycine and D-serine and the partial agonist D-cycloserine. Adjunctive administration of glycine (Buchanan et al., 2007), D-serine (Kantrowitz et al., 2015; Weiser et al., 2012) and D-cycloserine (Buchanan et al., 2007; Goff et al., 2008; Takiguchi et al., 2017) did not improve cognitive function, nor did D-serine when given in an attempt to boost cognitive remediation training (D'Souza et al., 2013). In a novel paradigm, Cain et al. (2014) examined the effect of intermittent weekly administration of D-cycloserine while patients took part in a 'Brain Fitness' programme in which an auditory discrimination task was practised 3-5 times a week. While the medication had no effect on traditional neuropsychological tests, learning of the auditory discrimination task was significantly enhanced compared with placebo, and this correlated with reduction in negative symptoms. The auditory discrimination task is thought to induce synaptic plasticity, a premise supported by the finding of increased intrinsic plasma D-serine levels following intense auditory discrimination training in association with improvement in memory and general cognition in patients with schizophrenia (Panizzutti et al., 2019). Together, these findings suggest a new, and possibly more meaningful, approach to the investigation of compounds that enhance NMDA receptor function in RCTs (Kantrowitz et al., 2018; Takiguchi et al., 2017).

Finally, L-carnosine is a dipeptide which increases the action of glutamate on NMDA receptors. An RCT of 75 patients showed improvement in a several measures of executive function and processing speed with small to medium effect sizes, suggesting that a larger trial is warranted (Chengappa et al., 2012).

\section{Nicotinic receptor agonists and modulators}

Slow information processing may be a fundamental abnormality underlying the generalised cognitive impairment in schizophrenia. Targeting its neural substrate is therefore a logical aim. Neural indices of information processing include measures of electrophysiological event-related potentials, such as the P50, during sensory gating paradigms. This reflects the ability of the brain to inhibit irrelevant sensory information and is robustly impaired in schizophrenia to the extent that it is considered an endophenotype (Earls et al., 2016). Animal studies show that P50 sensory gating is mediated by the action of inhibitory GABAergic interneurons on excitatory glutamatergic pyramidal cells. This mechanism is modulated by the action of acetylcholine on alpha7-nicotinic receptors present on both interneurones and pyramidal cells (Freedman, 2014). Decreased alpha7nAChR activity has been associated with impaired P50 and a series of studies has shown that alpha7nAChR agonists or modulators improve sensory gating in animal models (Jones, 2018). The rationale for studying alpha7nAChR agonists in schizophrenia (Ross and Freedman, 2015) is further bolstered by findings that the alpha7nAChR is coded by the CHRNA7 gene, which is deleted in some people with schizophrenia (Freedman, 2014), and by the unusually high rates of heavy nicotine use in patients, which possibly acts as a form of self-medication.
On the basis of this promising hypothesis, at least 13 alpha$7 \mathrm{nAChR}$ agonists or partial agonists have been developed and 12 have been tested in schizophrenia (Beinat et al., 2015). Three were discontinued after negative phase I studies (Beinat et al., 2015). Six others were tested in phase II studies, and after initial positive results were ultimately found to be ineffective and discontinued (Barch et al., 2016; Beinat et al., 2015; Haig et al., 2016; Kem et al., 2018; Lieberman et al., 2013; Tregellas et al., 2011; Umbricht et al., 2014; Walling et al., 2016).

Encenicline, a selective alpha7nAChR partial agonist, was found to improve non-verbal learning and memory, executive function and global cognition in two Phase II trials (Keefe et al., 2015; Preskorn et al., 2014) and thus proceeded to Phase III. Two multicentre international 26-week trials enrolled 1500 schizophrenia patients but the primary cognitive end point (MATRICS composite score) was not significantly different from placebo (https://www.alzforum.org/news/research-news/enceniclinemisses-endpoints-two-phase-3-schizophrenia-trials); neither study has been reported in a peer-reviewed journal.

There have been other studies of compounds with mixed pharmacological actions including alpha7nAChR agonism. Varenicline, currently used for smoking cessation, is a partial agonist of the alpha4beta2 nicotinic receptor and a full agonist at the alpha7nAChR. Several RCTs examining its effect on various measures of neuropsychological function in schizophrenia were negative (Hong et al., 2011; Shim et al., 2012; Smith et al., 2016).

Tropisetron is an alpha7nAChR partial agonist and 5HT3 antagonist. Although this showed normalisation of P50 in relation to improved memory after 10 days of treatment in non-smoking patients with schizophrenia (Zhang et al., 2012), a phase III extension of this study has not been reported and presumably was negative.

The alpha7nAChR shows rapid desensitisation to nicotinic agonists in animal models. This might explain why studies in which the drug has been administered acutely or for brief periods have proved positive for cognitive enhancement but not when the drug has been administered for longer intervals. It has been suggested that positive allosteric modulators (PAMs) of the alpha$7 \mathrm{nAChR}$ may prove more effective as they influence the receptor conformation in a way that enhances the effect of phasic release of the naturally occurring ligand, acetylcholine. RCTs of galantamine, an acetylcholinesterase inhibitor and PAM of alpha4 beta 2 and alpha7nACh receptors, have shown mixed results, being either entirely negative or only showing improvement in certain neuropsychological tests (Buchanan et al., 2008, 2017; Lee et al., 2007; Schubert et al., 2006).

Other studies have examined the effect of specific PAMs. For example JNJ-39393406 showed no effect on P50 sensory gating in schizophrenia and has been discontinued (Winterer et al., 2013). More recently, AVL-3288, has shown potentially positive effects on cognition in a healthy volunteer Phase 1 study (Gee et al., 2017). A study in patients has recently been completed and is yet to be reported (NCT02978599).

\section{Oestrogen receptor modulators}

Oestrogen has been shown in animal studies to be neuroprotective and to promote synaptic plasticity (Weickert et al., 2015). There is some evidence that sex hormone treatment improves symptoms in pre-and postmenopausal women with schizophrenia (Heringa 
et al., 2015). Oestrogen receptors are distributed throughout areas of the brain involved in cognitive function, and oestrogen receptor function has been shown to be altered in both men and women with schizophrenia (Weickert et al., 2015).

Direct treatment with oestrogen is hazardous because of the risk in women of breast and endometrial cancer. Raloxifene is a selective oestrogen receptor modulator which does not stimulate endometrial or breast tissue but is active on central nervous system and bone oestrogen receptors, being originally developed to prevent postmenopausal osteoporosis. It also has widespread actions as an agonist at 5HT, NMDA, AMPA, and D2 and D3 receptors. Although individual studies of raloxifene have shown mixed results, a meta-analysis of nine RCTs, including both female and male participants with schizophrenia, reported a beneficial effect on all PANSS subscales $(n=482$; total Hedges g 0.57 ; $95 \% \mathrm{CI}$, 0.41-0.99) (de Boer et al., 2018). Four of these assessed cognitive function, and while there was no overall beneficial effect on cognition, Huerta-Ramos et al. (2014) showed improvement in memory and verbal fluency in postmenopausal women, and Weickert et al. (2015) showed improvement in verbal memory and processing speed in both women and men. Neither study found a relationship between cognitive improvement and psychotic symptoms, suggesting a direct effect. However, Kulkarni et al. (2016) later found the opposite effect in women, with a significant improvement in clinical symptoms but not cognition. In a further analysis of their data, they stratified for menopause status and endogenous hormone levels and demonstrated a complex pattern with improvement or worsening of memory depending on menopausal stage: in pre and postmenopausal but not peri-menopausal women, verbal memory improved and semantic memory worsened (Gurvich et al., 2019). Finally, in a large study of raloxifene in 200 women with schizophrenia, no improvement was found in symptoms or cognition (Weiser et al., 2017). Weickert and Weickert (2017) examined, in detail, why the findings of these studies were so discrepant and concluded that differences in baseline symptom severity was the main explanatory factor, with patients with more severe symptoms being less likely to show cognitive improvement. They suggested that further studies should take the severity of baseline symptoms into account in their analysis.

\section{Antipsychotic medication}

Thus far, the findings of RCTs of novel compounds for cognitive dysfunction in schizophrenia are disappointing. Although there are some hints that modulation of the NMDA receptor may be an effective strategy, the results of the relevant studies are not robust enough to support any recommendation about the use of any of the tested compounds for adjunctive use with antipsychotic medication. For this reason, it might be worth reappraising the effect on cognition of the antipsychotic medications currently available. For example, in the CATIE study, quetiapine, olanzapine and risperidone had modest and equivalent beneficial effects on cognition. However, this effect was no greater than that of the FGA comparator, perphenazine, and was probably attributable to practice effects (Carpenter and Buchanan, 2008; Keefe et al., 2007). While this seems to put paid to any notion that SGAs directly improve cognition, there remains an argument that, because of deficiencies in trial design, there are hidden improvements in specific cognitive domains in subgroups of patients with antipsychotic medications with differing pharmacological profiles (Meltzer, 2015). Arguably this is borne out by a meta-analysis of RCTs of individual antipsychotic medications that found both positive and negative effects on different cognitive functions, depending on the particular medication being tested (Nielsen et al., 2015). In addition, an RCT using a novel trial design that compared haloperidol or flupenthixol (FGAs), with olanzapine, risperidone or aripiprazole (SGAs), found beneficial effects on cognition, with small to moderate effects with the SGAs at 6 and 24 weeks. The FGAs, having initially improved executive functions, showed a decline of moderate to large effect at 24 weeks. Finally, lurasidone, a new antipsychotic with potent antagonist activity at D2, 5-HT2A and 5HT7 receptors and partial agonist activity at $5 \mathrm{HT} 1 \mathrm{~A}$ receptors has been shown to improve cognitive function after 6 weeks, compared with placebo and quetiapine (Harvey et al., 2013). Thus, paying attention to the cognitive effects of individual antipsychotic medication pharmacological profiles may be a promising strategy as we enter an era of personalised medicine.

Dopamine is known to mediate important aspects of cognition, exemplified by the finding that unmedicated patients with prodromal Parkinson's disease have executive dysfunction (Weintraub et al., 2017). It is therefore highly likely that antipsychotic medication can add to the cognitive impairment already present in schizophrenia. Two studies have demonstrated this by comparing stable patients who had undergone guided dose reduction or discontinuation with matched groups remaining on maintenance treatment. The findings showed improvement on several tests of cognitive function without worsening of psychotic symptoms (Faber et al., 2012; Zhou et al., 2018).

\section{Recommendation for pharmacological management of cognitive impairment in schizophrenia}

- The best current pharmacological strategy for managing cognitive impairment in schizophrenia is to ensure, for each patient, that the lowest effective dose of antipsychotic medication is used. (B)

\section{Pharmacological strategies for comorbid affective disorder}

The 'Kraepelinian dichotomy' of bipolar disorder and schizophrenia has been under challenge for many years. The presence of mood symptoms, including depression and mania, is common in schizophrenia, as is the presence of positive symptoms in bipolar disorder. Diagnostic fluidity is a key component in first-episode psychosis; mood symptoms such as depression are particularly common in this early phase of illness (Herniman et al., 2019; Upthegrove et al., 2017). While the majority of firstepisode psychosis patients would meet diagnostic criteria for schizophrenia, for those patients without a clear schizophreniform presentation making a diagnosis is still essential for effective and prompt treatment (Marshall et al., 2014), as reviewed in the section on First-episode psychosis above, and multidisciplinary teams should be prepared to make and then if necessary change a diagnosis as the clinical presentation becomes clearer. Monitoring for significant mood symptoms in first-episode psychosis and also later in the course of schizophrenia is essential to develop appropriate treatment plans. 
Some limited biological evidence suggests potentially common aetiological pathways for both mood and positive symptoms, including systematic whole-genome linkage studies which have implicated chromosomal regions in common (Purcell et al., 2009), brain imaging studies with similarities in both structural grey and white matter (Cui et al., 2011) and functional abnormalities in some key areas including the hippocampus (Knöchel et al., 2014). But there is still distinction at a clinical and also brain network level; for example, people with schizophrenia may show a more generalised connective pattern dysfunction, whereas in bipolar disorder these may be more restricted to limbic and para-limbic networks (d'Albis and Houenou, 2014). Until contemporary models of SMI are robustly able to suggest clinically meaningful classifications of psychiatric disorders based on an identifiable pathophysiological process, it is likely that the schizophrenia/bipolar disorder dichotomy will remain in psychotic disorders and treatment guidelines need to reflect this.

\section{Prevalence, phenomenology and clinical importance of comorbid depression}

Depression as a discrete syndrome is relatively common in schizophrenia, with a prevalence in cross-sectional studies of around 30\% (Conley et al., 2007; Häfner, 2005; Häfner et al., 2005). This increases to over $50 \%$ if broadened to include depressive features or in longitudinal studies. Prevalence figures for depressive features are highest in the earlier phases of illnesses, being $40 \%$ in ARMS subjects and up to $80 \%$ in firstepisode psychosis patients (Yung et al., 2006; Upthegrove et al., 2010).

The phenomenological symptoms of depression in schizophrenia are broadly similar to those of major depressive disorder. However, some evidence suggests that negative cognitive appraisals of shame and loss are more prominent in post-psychotic depression as compared with major depressive disorders (Häfner, 2005; Sandhu et al., 2013), whereas in acute phases of psychosis, depression may be more related to the experience of positive symptoms themselves: perceived threat from powerful persecutors and/or malevolent hallucinations (Birchwood et al., 2005; Garety et al., 2001).

Depression in schizophrenia is associated with more frequent psychotic episodes (Buckley et al., 2009), increased duration of illness, substance use (Addington and Duchak, 1997), poor quality of life and suicide (Barnes et al., 1989; Reine et al., 2003; Saha et al., 2007). Depression during and after first-episode psychosis is the most significant risk factor for suicidal behaviour; 35 out of every 100 patients with first-episode psychosis have attempted suicide, and completed suicide is most common in these early years of illness (Ayesa-Arriola et al., 2015; Dutta et al., 2011; Upthegrove et al., 2010). In a systematic review and meta-analysis, depression after first-episode psychosis was also shown to have long-term impact on the likelihood of suicidal behaviour, lasting up to 7 years (McGinty et al., 2018). Depression in schizophrenia also has impact on systems outside of the individual and health care burden, with greater use of the mental health services and criminal justice system (Conley et al., 2007). However, the treatment of depressive symptoms in schizophrenia has not had the corresponding attention this evidence suggests is needed.

\section{Pharmacological management of depression in schizophrenia}

A meta-analysis by Whitehead et al. (2002) identified 11 appropriate trials in this field; however these studies had multiple methodological flaws including small sample sizes and absent reporting of allocation concealment, and few of the trials used an intention-to-treat strategy for the data analysis (Whitehead et al., 2003). The majority of studies assessed the effect of tricyclic antidepressants, which now would not be considered a first-line therapy for depression. The review concluded that co-prescribing antidepressants in schizophrenia may have some benefit; however, a fairer summary of the evidence might be that the case was 'unproven'.

There has been some strengthening of evidence more recently; Helfer and colleagues (2016) conducted a meta-analysis of studies addressing the safety and efficacy of antidepressants added to antipsychotic medication in the treatment of schizophrenia. Overall, adding an antidepressant to antipsychotic medication was found to be more efficacious than placebo or no intervention for the control of depressive symptoms, with a standardised mean difference across studies of $-0.25(95 \% \mathrm{CI},-0.38$ to -0.12$)$. When patients had more severe depression, the effect sizes were higher. Helfer et al. (2016) concluded that co-prescribing an antipsychotic medication and an antidepressant is a combination that may produce some beneficial effects. But their review had a broad focus, including all papers where antidepressants were coprescribed, and did not specifically aim to investigate the efficacy of antidepressant medication for the treatment of a comorbid depressive episode in schizophrenia.

Gregory et al. (2017) reported on a meta-analysis of the pharmacological treatment of depression in schizophrenia, focusing on studies where subjects had a formal diagnosis of depression at inclusion (as indicated by a standardised rating scale or diagnostic clinical interview). The pooled results from 26 studies provided moderate evidence that antidepressants are effective in the treatment of depression in schizophrenia, with an NNT of 5. Random effects meta-analysis of studies reporting end-point improvement in depression score also showed a significant improvement in favour of treatment. The majority of studies investigated SSRIs, with four studies investigating sertraline and four testing citalopram. Trials reporting results using the Calgary Depression Scale for Schizophrenia (CDSS: Addington et al., 1993), which is better able to differentiate depression features from negative symptoms than other standard depression rating scales, had larger effect sizes. This may indicate antidepressants are more effective when prescribed specifically for carefully assessed depression. However, the overall quality of studies investigating the effect of antidepressant medication to treat depression in schizophrenia remains moderate to poor; 19 out of 26 included articles would not meet current standards for reporting RCTs. In both the Helfer et al. (2016) and Gregory et al. (2017) meta-analyses there was no indication that any one class or individual antidepressant medication was superior.

Choice of antipsychotic medication. There is some limited evidence that SGAs may be less associated with depression than FGAs. Previously, depressive symptoms in schizophrenia were understood to be possibly the result of a depressogenic side effect of FGAs (Häfner, 2005), although this was 
not evidenced by placebo-controlled studies. Some research suggests SGAs have antidepressive effects, which can only partially be explained as occurring secondary to the improvement of positive and negative symptoms; rather there may be a direct (primary) effect on depressive symptoms (Möller, 2005; Zhou et al., 2015). Emsley et al. (2003) demonstrated in a sample of over 200 participants, using path analysis, that an improvement in symptom score with patients treated with quetiapine over haloperidol was largely mediated thorough an improvement in depression. Kim et al. (2007), in an open-label study, showed that switching from risperidone to amisulpride resulted in an improvement in depression score. Animal models of depression outwith schizophrenia have demonstrated some evidence for an antidepressant effect with quetiapine (McIntyre et al., 2007), which is approved in the United States for the treatment of resistant depression (Tundo et al., 2015). Both NICE and BAP guidance for the treatment of depression outlines clear benefit for the use of an SGA for augmentation of antidepressant medication for treatment-resistant depression (NICE 2007; Cleare et al., 2015). However, given the widespread use of SGAs, there has not been an evident reduction in the prevalence of depression in schizophrenia and thus their effect at a cohort level may be rather limited (Siris, 2000).

\section{Risks of co-prescribing antidepressants with antipsychotic} medication. Polypharmacy is not without risk in SMI, where patients may already have a heavy side-effect burden and poor physical health (Ballon and Stroup, 2013). Concomitant use of antidepressants with antipsychotic medication potentially leads to a further increased risk of cardiac effects, and additional ECG monitoring, including for prolongation of the QTc interval, may be required (Sicouri and Antzelevitch, 2018), particulary in the elderly (Rochester et al., 2018). In 2011, the MHRA issued guidance following concern about the possible dosedependent effects of the SSRI citalopram on QTc (see Funk and Bostwick, 2013; Vieweg et al., 2012), stating that combination with other medicines that also prolong QTc, including antipsychotic medication, was contraindicated (Medicines and Healthcare products Regulatory Agency, 2011). However, Tiihonen et al. (2012) reviewed co-prescribing of antidepressants with antipsychotic medication in a large population cohort and found that antidepressant use was not associated with a higher risk for mortality, although it was significantly associated with a decreased risk of completed suicide. Helfer et al. (2016) reported that adding an antidepressant to an antipsychotic regimen did not significantly increase rates of relapse in psychosis and did not increase the rate of drop out, but there was an increase in reporting abdominal pain, constipation, dizziness and dry mouth.

\section{Summary}

Recent systematic reviews and meta-analyses demonstrate qualified evidence that antidepressants may be effective in treating depression in people with schizophrenia. Observational studies also suggest that this combination does not cause a significant increase in adverse events. However, the evidence is clouded by the poor quality of relevant studies. Higher quality, large-scale RCTs are still needed. To ensure rigorous and conclusive results in future research, investigators should take account of the methodological limitations of the published studies in this area, apply specific depression inclusion criteria, use adequate methods of randomisation and allocation concealment, prioritise the appropriate measurement of depressive symptoms in schizophrenia, and report all adverse outcomes.

\section{Recommendations for the pharmacological treatment of comorbid depression}

- Clinicians would be justified in discussing an individual therapeutic trial of antidepressant medication with their patients with schizophrenia who are depressed. (A)

- Antidepressant medication should be discontinued if no benefit is found after an adequate trial. (B)

- Individual assessment of cardiovascular risk versus benefit will be needed and appropriate ECG monitoring should be in place for individuals at higher risk - for example, on high-dose or combined antipsychotic medication. (B)

- The choice of antipsychotic medication should be made following a full, careful assessment, considering the presence of depression in balance with all other factors: switching antipsychotic medication, for example from an FGA to an SGA, purely for the treatment of depression is not currently supported by strong evidence. (D)

\section{Prevalence, phenomenology and clinical importance of comorbid hypomania/mania}

While there has been some growth in research in the recognition and treatment of depression in schizophrenia, there has not been a concomitant increase in research into comorbid mania. Perhaps coloured by the even greater uncertainty around diagnosis than depression, mania in schizophrenia (schizomania) is largely investigated within schizoaffective disorder, a diagnostic category which has changed with time and location. DSM-IV recognises two subtypes of schizoaffective disorder: 'depressive type' and 'bipolar type', and requires that the mood symptoms must have been present for 'a substantial portion' of the course of the disorder, without further quantification (American Psychiatric Association, 1994). DSM-5 added a specification that symptoms of a major mood episode should have been present for the majority of the total duration of the active and residual portions of the illness, but does not qualify this with mania or depression (American Psychiatric Association, 2013). ICD10 likewise outlines that mood symptoms must be present for 'a substantial portion' of the course of the disorder, without further quantification (WHO, 1992). While the incidence of schizoaffective disorders varies over time and location as a result of the changes in diagnostic framework, it has been reported to be around $0.3 \%$ of the population (Lindenmayer and Kaur, 2016). In first-episode psychosis samples, the presence of mania is seen much less frequently than depression, being reported in between $5 \%$ and $15 \%$ of patients (Scott et al., 2016; Upthegrove et al., 2010). It is also reported, however, that schizoaffective disorder, which will include schizomania, is associated with an increased morbidity and mortality and a more complex clinical course than schizophrenia (Laursen et al., 2007). 


\section{Pharmacological management of hypomania/mania in schizophrenia}

Choice of antipsychotic medication. There have been very few studies that assess a differential pharmacological response for the bipolar subtype of schizoaffective disorder and none designed specifically for schizomania. After a systematic search, Lindenmayer and Kaur (2016) identified 14 studies, nine of which were doubleblind RCTs, investigating choice of antipsychotic medication for schizoaffective disorder. The majority include mixed samples of both schizophrenia and schizoaffective disorder, with only a few reporting subgroup analysis specifically for schizomania. One study reported a beneficial effect for paliperidone extended-release on acute mania symptoms, as measured by the Young Mania Rating Scale (YMRS), compared with placebo (Canuso et al., 2010). Fu et al. (2015) examined the efficacy of paliperidone extendedrelease in the maintenance of schizoaffective disorder, and also included the YMRS as an outcome measure. Again, paliperidone was more effective than placebo in preventing relapse of mania symptoms. Other RCTs of note featured in the review included two comparing antipsychotic medications: Zimbroff et al. (2007) found ziprasidone to be equally as effective as aripiprazole, while Kane et al. (2009) found no difference between oral aripiprazole as an adjunct to quetiapine or risperidone monotherapy in a subsample of patients with schizoaffective disorder (Kane et al., 2009). Thus, while there may be some limited evidence to support the use of paliperidone, other antipsychotic medications have no conclusive evidence or have not been tested specifically in schizomania, significantly limiting any conclusions regarding choice of antipsychotic medication.

Examining the evidence for the use of antipsychotic medication in bipolar mania may give some further limited information and a pragmatic framework for making individual decisions around choice of antipsychotic medication for patients with schizophrenia presenting with acute mania symptoms. BAP guidance on the treatment of bipolar disorder (Goodwin et al., 2016) reports data from clinical trials that suggest haloperidol, olanzapine, risperidone and quetiapine are effective in short-term reduction of symptoms of acute mania, and that aripiprazole may also be effective. In the maintenance phase of bipolar mania, evidence from RCTs suggests that olanzapine, quetiapine and risperidone long-acting injection are effective in preventing manic relapse.

Other medicines for acute mania: mood stabilisers. No studies have specifically been designed to assess the efficacy of antimanic agents in schizomania. A systematic review of relevant trials in schizoaffective disorder by Lindenmayer and Kaur (2016) found some with findings suggesting that, for both acute and maintenance treatment, an SGA prescribed alone may be just as effective as an SGA and mood stabiliser combined. However, most of the studies did not report separate results for those participants with schizomania. Analysing data from the ConstaTRE trial, which had a total sample of 666 patients with stable schizophrenia or schizoaffective disorder, Smeraldi et al. (2013) compared remission rates following randomisation to either risperidone LAI or oral quetiapine, and showed superiority for the risperidone LAI, with or without an adjunctive mood stabiliser.

Greenberg and Citrome (2015) reviewed studies of paliperidone in schizoaffective disorder, including two trials of acute schizoaffective disorder, which stratified randomisation as to whether subjects were being concurrently treated with a mood stabiliser (total of 614 participants, $69 \%$ of whom were receiving an adjunctive mood stabiliser, primarily valproate). The overall findings were that participants randomised to oral paliperidone extended-release showed a significant improvement compared with placebo and that no difference was seen between the adjunctive mood stabiliser and monotherapy treatment arms.

In summary, the study findings suggest that for the treatment of schizomania, the addition of a mood stabiliser to antipsychotic medication, which could be considered a relatively common management strategy, has very little evidence to support it. This uncertainty is primarily because only a few trials have focussed specifically on the treatment of schizoaffective disorder or reported data on a subsample with schizomania. Given this lack of relevant data, extrapolation of evidence on the treatment of mania in bipolar disorder may be of some help. In the treatment of bipolar mania, a number of antipsychotic medications, when combined with lithium or valproate, have been shown to be superior to monotherapy alone (Goodwin et al., 2016).

The lack of specific investigation into the treatment of schizomania or of schizoaffective disorder with stratification for mania significantly limits the clinical recommendations that can be made. There are no specifically designed, randomised, controlled, head-to-head trials of antipsychotic medication in schizoaffective disorder or schizomania, nor any trials designed to specifically assess the treatment of mania symptoms in schizophrenia. However, on a pragmatic basis the choice of antipsychotic medication will be balanced by both the presence of mania and other patient-specific symptoms. The severity and risk associated with positive or negative symptoms, risk of metabolic syndrome, cardiovascular risk and gender need to be considered. Following guidance on choice of antipsychotic medication, the presence of mania symptoms will be one of many considerations to weigh. Antipsychotic medication appears to be an effective strategy for relapse prevention; however, at present there is little evidence to suggest the primacy of any particular antipsychotic medication, and evidence for use of adjunctive mood stabilising medication in the treatment and prevention of schizomania is scarce.

\section{Recommendations for the pharmacological treatment of comorbid hypomania/mania}

- Choice of antipsychotic medication should be made on individual patient characteristics. (D)

- The use of an additional mood stabilising/anti-manic agent may be considered in an individual patient trial when mania symptoms persist after an adequate trial of an antipsychotic medication. (D)

- If an individual trial of a mood stabiliser medication proves to be ineffective, it should be discontinued. (D)

- Valproate prescription for women of childbearing potential should follow the mandatory requirements of the MHRA pregnancy prevention programme (see the section above on Recommendations regarding the pharmacological treatment of schizophrenia during pregnancy and the perinatal period). 


\section{Pharmacological strategies for comorbid substance misuse disorders}

Managing patients with schizophrenia who also use substances is a common and substantial challenge. While the focus is often on cannabinoids, comorbidity with nicotine and alcohol use is more common. Among individuals with schizophrenia there is a high prevalence of nicotine smoking (50-85\%) and other substance use (37-70\% in men) (De Leon and Diaz, 2005; McClave et al., 2010; Toftdahl et al., 2016). Tobacco smoking is a substantial contributor to the reduction in life expectancy for such individuals. The prevalence of stimulant use disorder, which can be associated with psychotic phenomena, has been reported to be higher in those with a diagnosis of schizophrenia spectrum disorder than in the general population, but it varies considerably depending on clinical setting, geographical location, and clinical differences between the study samples (Sara et al., 2015). Further, cannabis use disorder may frequently co-occur with stimulant use disorder (Sara et al., 2015), and the increasing use of novel psychoactive substances (NPS), for which objective testing is rarely possible, further complicates management.

Comprehensive and systematic reviews have described the difficulties faced in managing such comorbidity (Lubman et al., 2010; Wobrock and Soyka, 2009). Negative symptoms and cognitive impairment, particularly involving the prefrontal cortex, can make it challenging to engage an individual with schizophrenia in psychological approaches to reduce or prevent relapse of substance use, and may also contribute to poor medication adherence. Treatment guidelines (Marel et al., 2016; NICE, 2011b) recommend consideration of an individual's social and treatment context, the severity of both psychiatric and substance use disorder, and their readiness to change. Clear advice about harm minimisation should be given (e.g. from needles, poly-substance use, etc.) and patients reminded of the adverse consequences of their substance use.

There are few RCTs comparing different antipsychotic medications in patients with schizophrenia and comorbid substance use. Most of the evidence derives from small, low-quality studies with uncontrolled designs, describing the impact of switching antipsychotic medication, or case series. The exception to this is the CATIE study (Lieberman et al., 2005); 61\% of the participants reported smoking, and according to clinicians' ratings, 35\% used alcohol and $23 \%$ used illicit drugs, including $37 \%$ who met the criteria for a current substance use disorder (Mohamed et al., 2015; Swartz et al., 2006). In contrast to the overall study results, comorbid illicit drug use attenuated the superiority of olanzapine over the other antipsychotic medications is used in the study (Swartz et al., 2008). While there were reductions in substance use evident in all treatment groups, no significant differences emerged between the different antipsychotic medications on measures of smoking or alcohol or drug use (Mohamed et al., 2015).

There are case reports and series describing beneficial effects of clozapine on reducing substance use (Lingford-Hughes et al., 2012). However, there has been no randomised prospective trial of clozapine in this clinical population.

\section{Substance-specific pharmacotherapy}

Nicotine. Individuals with schizophrenia are three times more likely to initiate smoking, and although they are equally as interested in quitting smoking and taking medication (including nicotine replacement therapy: NRT) they are five times less likely to be successful in stopping smoking than the general population (De Leon and Diaz, 2005). It is therefore important that support to stop or change their smoking behaviour should be offered. The role of e-cigarettes in helping such individuals change remains unclear.

In a meta-analysis of efficacy and tolerability of adjunctive pharmacotherapy for smoking cessation in adults with schizophrenia, schizoaffective disorder and bipolar disorder, both bupropion and varenicline were superior to placebo with no differences in tolerability (Roberts et al., 2016). However, the quality of trials was noted as low. Systematic reviews of varenicline have concluded that it is superior to placebo in smoking cessation in individuals with SMI (Cahill et al., 2016; Roberts et al., 2016). Varenicline increased the chances of successful long-term smoking cessation between 2-3 times compared with pharmacologically unassisted attempts to quit (Cahill et al., 2016). While an analysis of reports to the FDA suggested that varenicline is associated with a substantially increased risk of depression and suicidal/self-injurious behaviour (Moore et al., 2011), subsequent meta-analyses and systematic reviews of relevant RCTs have found no clear evidence of an increased risk of neuropsychiatric or other adverse events for varenicline, compared with placebo (Thomas et al., 2015, Wu et al., 2016). Specifically, Thomas et al. (2015) did not find a significant difference in risk of suicide, attempted suicide or suicidal ideation, depression or death.

The Evaluating Adverse Events in a Global Smoking Cessation Study (EAGLES; Anthenelli et al., 2016) investigated the neuropsychiatric complications of varenicline treatment in over 8000 motivated-to-quit individuals with and without a psychiatric disorder. It reported that there was no significant increase in neuropsychiatric adverse events attributable to varenicline or bupropion relative to nicotine patch or placebo in all individuals. Varenicline was more effective than placebo, nicotine patch, and bupropion in helping smokers with a psychiatric disorder (including psychosis) achieve abstinence, and bupropion and nicotine patch were more effective than placebo. The majority of trials only included participants who were motivated to quit and whose psychiatric disorder was stable, so results may not be generalisable to all patients. Those who are motivated to stop smoking should be told about the small chance they may experience severe mood changes and all clinicians should be alert for these changes.

Alcohol dependence. Usual medication regimens for alcohol detoxification can be used in patients with schizophrenia comorbid with alcohol dependence, with due consideration being given to the doses required to avoid complications, such as seizures, and taking into consideration any pharmacokinetic or pharmacodynamic interactions with other prescribed medicines. Patients with schizophrenia are more likely to require inpatient admission for detoxification and so relapse-prevention plans should be established and implemented at this time (Lingford-Hughes et al., 2012).

There are three main relapse-prevention medications to be considered for patients with alcohol dependence; acamprosate, naltrexone and disulfiram (NICE CG115, 2011c). There is limited evidence for their use in patients with comorbid schizophrenia, but the principle of active treatment of alcohol dependence as part of the overall patient management plan is key (LingfordHughes et al., 2012). Data relating to the pharmacokinetics of 
these medications as well as safety data can be used to extrapolate from the treatment of alcohol dependence alone to help determine the risk-benefit profile of treatment with a specific medication for an individual patient (Sinclair et al., 2016).

For both acamprosate and naltrexone, no adverse effects of particular relevance for patients with schizophrenia compared with non-comorbid populations have been noted. A placebo-controlled RCT in patients with psychosis reported that naltrexone (an opiate antagonist) was associated with fewer drinking days, fewer heavy drinking days and less craving, though psychosis did not concurrently improve (Petrakis et al., 2006).

Use of disulfiram in patients with psychosis is relatively contraindicated as its blockade of dopamine-beta-hydroxylase results in higher dopamine levels, which could potentially acutely increase psychosis, and was observed to do so in early studies where it was prescribed at much higher doses (Sinclair et al., 2016). However, more recent studies in patients with psychosis, not all of whom had schizophrenia, reported that disulfiram (200-250 mg per day) reduced alcohol consumption with only limited adverse events (Lingford-Hughes et al., 2012; Petrakis et al., 2006).

Cocaine and other stimulants. There have been many studies exploring pharmacotherapeutic interventions for cocaine dependence, although these have not been in samples of people with schizophrenia. These studies have failed to demonstrate robust effectiveness for any medication. Psychosocial approaches, particularly contingency management, are the most effective strategy in non-comorbid populations who use stimulants. Consequently, it is appropriate to use similar approaches in patients with schizophrenia.

Opioids. Opioid use (e.g. heroin) in patients with schizophrenia is less common than alcohol or cannabis (Toftdahl et al., 2016). The common substitute medications are methadone or buprenorphine and for relapse prevention, naltrexone. Methadone prolongs the QTc and it is advisable to check the ECG of anyone prescribed methadone who is also receiving an antipsychotic medication. In addition, tolerance to methadone is lost relatively rapidly, and for those patients who are disorganised, supervised consumption is required, potentially in the long term.

Cannabis. Of the few studies reporting treatment of schizophrenia comorbid with cannabis use, no single SGA has been shown to have superiority over another. For example, olanzapine and risperidone have a similar impact on substance use and psychosis (Sevy et al., 2011; van Nimwegen et al., 2008). A small pilot study compared clozapine (mean: $225 \mathrm{mg} / \mathrm{d}$ ) with ziprasidone (mean: $200 \mathrm{mg} / \mathrm{d}$ ) in patients with schizophrenia and cannabis use disorder and found that cannabis use similarly decreased in both groups during the 12-month follow-up (Schnell et al., 2014). Clozapine was associated with a greater reduction in positive symptoms but more side effects.

Other substances. The increase in the use of NPS poses a particular challenge in the management of patients with schizophrenia. Increasingly clinicians will need to rely on the history, and empirical observation of the psychopharmacological properties of a substance on the patient's presentation, as objective testing is generally not possible. There is no evidence to support the use of specific drug-targeted medication in treating NPS use, but shortterm symptomatic treatment may be needed, for example benzodiazepines for insomnia during withdrawal.

\section{Recommendations regarding the} pharmacological management of comorbid substance use in schizophrenia

- A comprehensive assessment, including why and how substances are taken, should be undertaken routinely in all patients. (D)

- Antipsychotic medication should be optimised and clozapine considered in patients with persisting substance use disorder. (C)

- Support to stop or change smoking behaviour should be offered to all patients. (A)

- All patients quitting smoking should be told about the small chance that they may experience severe mood changes and all clinicians should be alert for these changes. (C)

- Treatment focussed on managing comorbid substance use should be offered. While psychosocial approaches underpin management, pharmacotherapy should be actively considered and offered where possible, for example, nicotine substitution/withdrawal, optimising opioid substitution, alcohol detoxification and relapse prevention. (A)

\section{Pharmacological intervention for persistent aggression in schizophrenia}

The evidence base for pharmacological intervention for persistent aggression and violence in people with schizophrenia is weak and no medication has yet been approved by regulatory agencies for the treatment of such behaviour.The relevant studies have tested treatments with limited efficacy and have had methodological shortcomings, including small sample sizes, inadequate definition of the outcome variable and low outcome rates. Further, the target population of patients, characterised by aggression and hostile behaviour, may be less likely to be recruited, provide consent, or maintain their participation in a study.

Clinicians considering the aetiology of aggression and violent behaviour in people with schizophrenia may recognise subtypes, for example, cases where aggression is judged to be delusionally driven, where impulsivity is a major contributor, where there are co-occurring personality disorder features, and in the context of comorbid substance use; cognitive deficits and underlying somatic conditions can also be risk factors (Citrome and Volavka, 2011, 2014; Nederlof et al., 2013). While the efficacy of medications might be postulated to vary across these subtypes of aggressive, violent behaviour, clinical trial samples are seldom, if ever, selected in this regard (Citrome and Volavka, 2014).

Clinicians commonly add a benzodiazepine, lithium or anticonvulsant medication to continuing antipsychotic medication in the hope of reducing persistent aggression and the risk of violence. However, the evidence that such adjunctive strategies provide long-term benefit in terms of a reduced risk of aggressive behaviour is inconsistent and uncertain (Citrome and 
Volavka, 2014). Adjunctive valproate, particularly, has a long history of being prescribed for aggressive behaviour in people with schizophrenia as well as those with other psychiatric diagnoses, such as bipolar disorder and personality disorder (Lindenmayer and Kotsaftis, 2000). Despite this widespread use, there are no double-blind studies of valproate in schizophrenia for this indication, although there are a few open studies. For example, an 8-week, open-label, randomised trial compared risperidone monotherapy with a combination of risperidone and valproate for hostility in people with schizophrenia, selected on the basis of having exhibited poor impulse control and/or aggressive or hostile behaviour. The results failed to demonstrate any meaningful advantage for the combination (Citrome et al., 2007). Nevertheless, a Cochrane systematic review (Wang et al., 2016) concluded that the limited evidence from open RCTs suggested some efficacy for the augmentation of antipsychotic medication with valproate for excitement and aggression. Volavka and Citrome (2011) concluded, similarly, that adding valproate or carbamazepine to antipsychotic treatment might reduce impulsive aggression in particular patients, but they recommended that it should only be prescribed as an individual trial, with close monitoring of effectiveness.

\section{Antipsychotic medication for persistent aggression}

The relative risk of violence against others is higher in people with schizophrenia and related psychoses than in the general population (Fazel et al., 2009). Nevertheless, those who are free of psychotic symptoms, such as paranoid thoughts or command auditory hallucinations, may be no more likely to be aggressive or violent than people without a mental disorder (Friedman, 2006) and are probably more likely to be victims of violence than perpetrators (Brekke et al., 2001; Monahan et al., 2017). While a specific anti-aggression effect has not been consistently demonstrated for antipsychotic medication in general (Citrome et al., 2001 Volavka and Citrome, 2008), greater adherence to continuing antipsychotic medication by people with a psychotic illness, and therefore presumably better symptom control, appears to be associated with reduced levels of aggression (Arango et al., 2006; Grinshpoon et al., 1998) and a lower likelihood of violence and crime (Fazel et al., 2014; Nilsson and Falk, 2015). However, there does not seem to be much justification for using high-dose antipsychotic medication for persistent aggression, at least on the basis of findings in psychiatric intensive care settings (Choong et al., 2011; Herlihy et al., 2010).

Clozapine for persistent aggression. Although in the CATIE study, olanzapine was found to have advantages in terms of a specific anti-hostility effect over the other antipsychotic medications tested (Volavka et al., 2014), the antipsychotic with the best evidence for an anti-hostility effect in schizophrenia is clozapine (Citrome and Volavka, 2014; Krakowski et al., 2006; Victoroff et al., 2014). Clozapine has long been recognised as having such properties, possibly linked to its serotonergic effect (Hector, 1998; Volavka and Citrome, 1999; Wilson and Clausen, 1995). A systematic review of relevant studies (Frogley et al., 2012), including four RCTs, concluded that clozapine is an effective medication for the management of aggression and violent behaviour in patients with schizophrenia, perhaps particularly for treatment-resistant illness. It may also have a role in the treatment of such refractory problems in forensic psychiatric populations (Hotham et al., 2014; Patchan et al., 2018; Stone-Brown et al., 2016).

The reduction in hostility and aggression with clozapine found in RCTs (Citrome et al., 2001; Krakowski et al., 2006) has often appeared to be independent of improvement in positive symptoms, or side effects such as akathisia or sedation. However, it is unclear whether this means that clozapine has a specific antihostility effect, as has been postulated (Buckley et al., 1995; Citrome et al., 2001). For example, the observation that clozapine treatment may be associated with a decrease in hostility despite little apparent change in the severity of psychotic symptoms does not preclude some relief of the affective impact of positive symptoms, which may have underlain the aggressive behaviour (Taylor and Estroff, 2003).

\section{Recommendations for the pharmacological management of persistent hostility/ aggression}

- A trial of clozapine should be considered for people with schizophrenia characterised by persistent symptoms of aggression and hostility. (B)

- In clinical practice, the initiation of an augmentation strategy to tackle persistent, aggressive, behaviour should be targeted towards the perceived cause of the behaviour, treated as an individual medication trial, and the risks and benefits appropriately monitored and reviewed. (S)

- The aim of pharmacological treatment in this context may be to tackle symptoms that are limiting a patient's ability to engage in a suitable psychological intervention, which could be focussed on the treatment of aggression and hostile behaviour. (S)

\section{Treatment-resistant schizophrenia}

The definition of treatment-resistant schizophrenia (TRS) has recently been operationalised by the Treatment Response and Resistance in Psychosis (TRRIP) working group (Howes et al., 2017), drawing on existing definitions by the National Institute for Health and Care Excellence (NICE, 2014a), the American Psychiatric Association (APA, Lehman et al., 2004) and the World Federation of Societies of Biological Psychiatry (WFSBP, Hasan et al., 2012). All are consistent in defining treatment resistance as a failure to respond to two trials of antipsychotic medication, of adequate dose and duration. In routine clinical practice, between $20 \%$ and $40 \%$ of people thought to have a treatmentresistant illness will have low or undetectable plasma antipsychotic levels, probably due to poor adherence (McCutcheon et al., 2015, 2018). The TRRIP criteria emphasise the importance of confirming adherence to treatment, ideally by measuring plasma concentration or by using a depot/LAI antipsychotic preparation (Howes et al., 2017).

It remains uncertain whether TRS should be considered simply as the more severe end of the illness spectrum or as a distinct subtype of schizophrenia for which neurobiological correlates of treatment resistance should be explored (Gillespie et al., 2017). Recently, there has been increasing interest in whether it is 
meaningful to further subdivide TRS into early and late-onset resistance. At the onset of illness, rates of primary treatment resistance have been shown to be up to $23 \%$ (Lally et al., 2016). Less commonly, treatment resistance develops over time, after an initial response (Demjaha et al., 2017). There has been some speculation that these patterns of TRS may have different aetiologies, but it is too early to make any treatment recommendations on the basis of such a distinction.

One limitation of the evidence base for prescribing is the difficulty of defining response in clinical trials involving people with TRS. Since remission criteria are rarely achieved in TRS patients, the response threshold criterion has tended to be a $20 \%$ or greater reduction in total BPRS/PANSS score (Siskind et al., 2017), which may fall short of a clinically relevant change. As noted above, in the section on Acute psychotic episode, a reduction in total PANSS score of at least $20 \%$ in acutely ill, psychotic patients corresponds to a CGI rating of 'minimally improved', the smallest improvement considered clinically meaningful (Leucht et al., 2005, 2006). Applying this threshold criterion, the response rate for clozapine is around 40\% (Siskind et al 2017). A common clinical observation is that for most patients with TRS there is some positive response to clozapine, although it seems that this is not always well captured by change in PANSS score. Overall symptom severity may be less relevant in this group than other outcomes, such as a reduction in 'target symptoms' that are causing distress or disability, an improvement in disturbed behaviour, or increased level of engagement in therapeutic and social activities. It is important to bear in mind that even a modest symptom reduction in a patient who is otherwise resistant to treatment may be of functional significance, especially if sustained over many years.

\section{Clozapine for treatment-resistant schizophrenia}

Clozapine is the only drug licensed for TRS in the UK. There is consistent evidence that clozapine is the most effective antipsychotic for TRS (Siskind et al., 2016); a response to clozapine has been found in $30-75 \%$ of people with schizophrenia that has failed to respond to other antipsychotic medications (Agid et al., 2011; Essali et al., 2009; Kane, 1992; Siskind et al., 2017). There are claims for other benefits with clozapine, including improvement in cognitive function (Bilder et al., 2002; Woodward et al., 2005), a low liability for, and treatment of, TD (Ricciardi et al., 2019), relief of care-giver burden (Conley, 1998), a decrease in cigarette smoking (de Leon et al., 2005) and possibly less requirement for adjunctive medications (Chong et al., 2000; Glick et al., 2004), as well as an anti-hostility effect (see section on Pharmacological intervention for persistent aggression in schizophrenia).

Large pragmatic clinical trials, such as CATIE Phase 2 (McEvoy et al., 2006) in the USA and the CUtLASS 2 study (Lewis et al., 2006) in the UK, compared the effect on TRS of switching to either clozapine or a non-clozapine SGA. CUtLASS 2 found that when treating schizophrenia that had shown a poor response to sequential trials of two or more antipsychotic medications, there was an advantage to commencing clozapine rather than another SGA in terms of symptom improvement over a year. Similarly, CATIE Phase 2 found that for people whose illness had prospectively failed to improve with SGA treatment, clozapine proved to be more effective than switching to another SGA. The primary outcome measure for this study was time to discontinuation from the assigned SGA for any reason, and this was significantly longer for clozapine than for quetiapine or risperidone, although not olanzapine. Further, by 3-month follow-up, PANSS total scores had decreased more in patients receiving clozapine than in those treated with quetiapine or risperidone, but not those assigned to olanzapine. Thus, olanzapine appeared to have a modest advantage over the other non-clozapine SGAs, although the findings may be confounded by the relatively high dosage of this drug used: mean modal daily doses were clozapine $332 \mathrm{mg}$, olanzapine $23.4 \mathrm{mg}$, quetiapine $642.9 \mathrm{mg}$ and risperidone $4.8 \mathrm{mg}$. The recommended daily dose range for olanzapine is $10-20 \mathrm{mg}$, and there are case reports and studies suggesting that doses of olanzapine above $20 \mathrm{mg}$ may be effective for TRS (Citrome and Kantrowitz, 2009; Meltzer et al., 2008; Roth, 2008). However, there does not seem to be any advantage to high-dose olanzapine in non-TRS (Kinon et al., 2008), and a relevant systematic review showed no advantage for olanzapine over clozapine in TRS, even when high-dose studies were included (Souza et al., 2013; see also section on High-dose olanzapine for treatment-resistant schizophrenia).

As Goodwin et al. (2016) comment in the BAP guidelines on bipolar disorder, large-scale observational studies over long periods can be more convincing and of a higher quality than many RCTs, with superior external validity. In addition, such pharmaco-epidemiological studies are generally free of industry bias. Pharmaco-epidemiological studies have consistently shown that clozapine treatment is associated with a reduction in all-cause mortality (Cho et al., 2019a; Wimberley et al., 2017), and this seems to be driven by a reduction in suicidality (Meltzer and Okayli, 1995; Meltzer et al., 2003; Wimberley et al., 2017). Clozapine has been shown to reduce admission rates compared with other antipsychotics (Kesserwani et al., 2019) and in within-patient mirror-image studies (Siskind et al., 2019), and a recent meta-analysis showed lower admission rates under clozapine than any other drug, including LAIs (Land et al., 2017). Clozapine treatment is also associated with lower rates of treatment failure than any other oral antipsychotics (Tiihonen et al., 2017). As noted above (see section on Pharmacological intervention for persistent aggression in schizophrenia), there is growing pharmaco-epidemiological evidence for the clinically observed anti-hostility action of clozapine, including a reduction in convictions for violent crime (Bhavsar et al., 2019; Fazel et al., 2014).

It is notable that these benefits on mortality, readmission rates and crime can best be detected with long-term follow-up, and are therefore often not seen in RCTs, with their short follow-up times. It is important to weigh up the consistent evidence favouring clozapine from pharmaco-epidemiological and pragmatic studies against the finding of only modest benefits in RCTs (Siskind et al., 2016).

Clozapine is associated with an increased risk of severe, potentially life-threatening side effects, including myocarditis (see section on Cardiovascular disease and deaths in schizophrenia and antipsychotic medication), cardiomyopathy, and agranulocytosis, compared with other commonly used antipsychotic medications (Berk et al., 2007; Layland et al., 2009; Li et al., 2019; Schulte, 2006), but these are still rare events. Sedation, hypersalivation and constipation are much more common and problematic for patients. 
Despite the benefits of clozapine on reducing all-cause mortality, psychiatrists may be reluctant to use clozapine, partly due to a lack of experience and knowledge of the drug, and fear of adverse effects (Nielsen et al., 2010), and lack of resources for monitoring (Gee et al., 2014). Furthermore, psychiatrists frequently discontinue clozapine in the early stages of treatment (Legge et al., 2016), and there is evidence that many of these discontinuations could be avoided (Nielsen et al., 2013b) if psychiatrists were better educated about common adverse effects and their treatment.

Optimising clozapine treatment. Despite the robust evidence for efficacy in TRS, the therapeutic response may be disappointing; even with optimum clozapine treatment, only $30-60 \%$ of patients with TRS will respond satisfactorily (Chakos et al., 2001; Iqbal et al., 2003; Siskind et al., 2017). However, the proportion of responders might be higher if less time were spent testing medication strategies with a lack of convincing evidence for efficacy for treatment-refractory schizophrenia, delaying the start of clozapine (Howes et al., 2012a; Shah et al., 2018; Yoshimura et al., 2017).

Should the therapeutic response to clozapine prove to be disappointing, before other treatment options are considered, adherence should be checked and treatment optimised, including the systematic assessment and management of side effects (Beck et al., 2014; Yusufi et al., 2007). A range of other factors that may be contributing to apparent clozapine resistance, such as continuing substance use, lack of psychosocial intervention and unrecognised mood disturbance, should be considered and addressed. Evidence from clinical trials suggests an advantage to longer treatment with clozapine, although such a conclusion should be qualified as different definitions of improvement are used. The period recommended varies from 4 to 12 months (Buchanan et al., 2010; Conley et al., 1998; Meltzer, 1992). Schulte (2003) reviewed studies of plasma clozapine concentration monitoring and the time to response and concluded that an adequate trial should be at least 8 weeks. However, response may be delayed longer in a proportion of patients; this may reflect the fact that it takes some weeks to reach an optimum dose that balances efficacy with adverse effects. Meltzer (1992) concluded that $30 \%$ of patients treated with clozapine would respond by 6 weeks, another $20 \%$ by 3 months and a further $10-20 \%$ by 6 months. On this basis, Kerwin and Bolonna (2005) considered it would be reasonable to test clozapine monotherapy for 6 months.

Therapeutic drug monitoring may be helpful for assessing medication adherence and drug-drug interactions, and also when titrating clozapine dosage to ensure an adequate plasma concentration is reached. The target plasma clozapine concentration to ensure an adequate trial is generally considered to be $350 \mathrm{mg} / \mathrm{L}$, although clinical response is commonly seen at lower levels (Couchman et al., 2013; Dettling et al., 2000). Factors influencing clozapine plasma concentration include the daily dose, caffeine (raises plasma level), age, gender, the co-prescription of drugs such as phenytoin and other cytochrome P450 enzyme inducers (reduce plasma level) and cigarette smoking (Rostami-Hodjegan et al., 2004). Smoking tobacco (but not chewing tobacco, nicotine patches, inhalers or e-cigarettes) mobilises polycyclic aromatic hydrocarbons, which leads to an increased rate of clozapine metabolism and a corresponding decrease in the plasma level. Rostami-Hodjegan et al. (2004) provided nomograms derived from large samples of male and female patients receiving clozapine, which illustrate the relationship between such factors and clozapine plasma level. These can be helpful in the clinical management of individual patients, to guide clozapine dose adjustment and assess medication adherence. Referring to these nomograms, the extent of the effect of smoking in decreasing plasma clozapine concentration is demonstrated. On average, the clozapine dose requirement will be around $50 \%$ greater in smokers (Couchman et al., 2010). If a patient stops smoking, there is a risk of toxicity with serious complications such as seizures unless the clozapine dose is promptly and appropriately reassessed. Mean plasma clozapine tends to rise after the age of 65 years so the dose should be reduced accordingly in elderly patients (Bowskill et al., 2012)

The ratio of clozapine:norclozapine plasma concentration can also provide information relevant to dosage adjustment. Norclozapine is a potentially active metabolite of clozapine, but has a longer plasma half-life. The mean clozapine:norclozapine ratio is around 1.33, across the dose range. A ratio of less than 0.5 raises the possibility of erratic adherence in preceding days while a value greater than 3 suggests that the blood draw was not a trough ( $12 \mathrm{~h}$ post-dose) sample or that clozapine metabolism is saturated, either because of the dose prescribed or because there is inhibition of clozapine metabolism due to an interaction with concurrent medication, such as fluvoxamine or ciprofloxacin (Couchman et al., 2010; Flanagan et al., 2003).

Abrupt withdrawal of clozapine should be avoided, except in severe adverse reactions. Suddenly stopping clozapine therapy can precipitate a range of symptoms, such as nausea, vomiting, diarrhoea, sweating and headache (Durst et al., 1999; Shiovitz et al., 1996). These are generally considered to be cholinergic rebound phenomena. Relatively severe dystonias, dyskinesias and catatonic symptoms may also be provoked by rapid withdrawal of clozapine (Ahmed et al., 1998; Koychev et al., 2016). Further, a minority of patients may develop a withdrawal psychosis, characterised by delusions, hallucinations, hostility and paranoid reactions, which may be more severe than observed previously (Ekblom et al., 1984; Goudie, 2000; Tanríverdi and Yazící, 1996). If clozapine is re-introduced to treat the withdrawal psychosis, the dose required to achieve remission may be substantially higher than that initially prescribed (Miodownik et al., 2006).

Clozapine augmentation with another antipsychotic. If an adequate trial of clozapine monotherapy proves to be of limited efficacy, augmentation strategies may be considered, although few of these drug combinations have a clear rationale and none has a convincing evidence base. Clinical studies have most commonly tested the value of adding another antipsychotic medication. Risperidone has been most often tested as an adjunct to clozapine in RCTs (Akdede et al., 2006; Anil Yağcioğlu et al., 2005; Freudenreich et al., 2007; Honer et al., 2006; Josiassen et al., 2005), but RCTs have also investigated the addition of amisulpride (Assion et al., 2008; Barnes et al., 2018), sulpiride (Shiloh et al., 1997) or aripiprazole (Chang et al., 2008; Fleischhacker et al., 2010) to clozapine, and there are relevant open trials and case reports regarding augmentation with ziprasidone (Henderson et al., 2009; Ziegenbein et al., 2005). The evidence from these studies is inconsistent. For example, a Cochrane review (Barber et al., 2017) examined five RCTs testing clozapine combined with haloperidol, aripiprazole, amisulpride, quetiapine, sulpiride, 
ziprasidone and risperidone. It proved not to be possible to reach a conclusion as to whether any particular combination strategy was superior to another, partly because of the methodological limitations of the eligible studies. Another Cochrane systematic review specifically addressed augmentation of clozapine with sulpiride (Wang et al., 2010). Three eligible short-term trials and one long-term trial were found, all involving participants with schizophrenia that was either treatment resistant or with prominent negative symptoms. The conclusion was that while sulpiride plus clozapine may be more effective than clozapine alone in producing clinical improvement in such a context, more robust data are required. Meta-analyses of larger numbers of relevant clinical trials have concluded that the expected benefit of augmenting clozapine with a second antipsychotic mediation is at best modest, and may not be evident until at least 10 weeks of treatment (Barbui et al., 2009; Paton et al., 2007; Taylor et al., 2012). With regard to the nature of the possible benefit, there are some indications that such a strategy may be more effective for the reduction of negative symptoms rather than positive symptoms (Barnes et al., 2018; Chang et al., 2008; Josiassen et al., 2005).

In relation to the potential risks, RCTs and open studies have generally found clozapine augmentation with a second antipsychotic to be relatively well tolerated. The main treatment-emergent side effects were predictable from the pharmacology of the augmenting drug, with EPS and prolactin elevation emerging as the most common problems. Nevertheless, comprehensive assessment of side effects in the trial of clozapine augmentation with amisulpride conducted by Barnes et al. (2018) revealed that those participants in the amisulpride group had a significantly greater burden of adverse events, which were more likely to be cardiac symptoms. Serious adverse effects reported with adjunctive risperidone include agranulocytosis, atrial ectopics and possible NMS (Chong et al., 1997; Godleski and Serynak, 1996; Kontaxakis et al., 2002), while augmentation with aripiprazole has been associated with nausea, vomiting, insomnia, headache and agitation in the first 2 weeks (Ziegenbein and Calliess, 2006), as well as tachycardia (Chang et al., 2008) and modest weight loss (Karunakaran et al., 2007; Ziegenbein and Calliess, 2006). These findings may have implications for the safety and tolerability monitoring of such medication combinations, including vigilance for indicators of cardiac abnormalities.

One possible criterion guiding the choice of the augmenting antipsychotic medication is a complementary receptor profile to that of clozapine, that is, a potent D2 dopamine receptor blocker as clozapine is a relatively weak D2/3 blocker (Genç et al., 2007; Kontaxakis et al., 2005). Additional criteria might include a relatively low liability for extrapyramidal side effects, prolactin elevation, and a low risk of compounding characteristic side effects associated with clozapine such as sedation, weight gain and other metabolic problems (Josiassen et al., 2005). Use of these criteria might partly explain why clinicians commonly choose amisulpride, aripiprazole and sulpiride to augment clozapine (Molina et al., 2009; Paton et al., 2007).

\section{Recommendations regarding clozapine treatment for treatment-resistant schizophrenia}

- Clozapine should be the first choice of treatment for patients with schizophrenia whose illness has shown a poor response to sequential trials of two (or more) different antipsychotic medications that have been adequate in terms of dosage and duration. (A)

- Poor medication adherence and comorbid substance use should be excluded as causes of the apparent poor response to antipsychotic medication. (A)

- Where a patient's illness meets the criteria for treatmentresistant schizophrenia, a trial of clozapine should be considered with minimal delay. (B)

- The treatment plan for individual patients should ensure adherence with the recommended starting dose titration schedule and the requirements for laboratory investigations and side-effect monitoring, as well as meeting the patient's need for support from the clinical team, relatives and carers. (S)

- For an adequate trial, clozapine monotherapy should be prescribed for at least 6 months. (B)

- Treatment-emergent side effects should be monitored, preferably using a self-report instrument such as the GASS-C (Hynes et al., 2015). (S)

- Adverse effects should be managed proactively, to maximise tolerability and reduce the risk of discontinuation. (S)

- Consideration should be given to the use of clozapine plasma concentration to guide dosage and check adherence where there is a sub-optimal response, or where adverse effects are problematic. (D)

- If possible, when a patient's clozapine is to be discontinued, the dose should be gradually tapered over at least 1-2 weeks. (B)

- After stopping clozapine, particularly if stopped abruptly (e.g. because of agranulocytosis), a patient's physical and mental state should be monitored for symptoms reflecting cholinergic rebound or rapid re-emergence of psychosis, particularly in the first week. (B)

- If clozapine therapy is temporarily interrupted for more than $48 \mathrm{~h}$, it must be restarted at a dose of 12.5 $25 \mathrm{mg} /$ day. If this dose is well tolerated, with no cardiovascular or respiratory symptoms, and previous standard dosage titration has been uneventful, then it may be reasonable to titrate the dose to the therapeutic level more rapidly than is recommended for initial treatment (Clozaril SmPC; Nikolic et al., 2019). If a patient previously experienced adverse effects with initial dosing, even if a therapeutic dose was subsequently successfully achieved, rapid re-titration should be avoided. (S)

- Augmentation strategies with clozapine should only be considered after optimised clozapine treatment has been administered for an adequate period of not less than 3 months. (S)

- Clozapine augmentation with a second antipsychotic: an adequate trial of clozapine augmentation with another antipsychotic medication may need to be at least 10 weeks in duration. (B)

- When choosing an augmenting antipsychotic medication, consideration should be given to medications with a complementary receptor profile to clozapine, and a side-effect profile that minimises compounding recognised problems with clozapine such as sedation, weight gain and metabolic side effects. (B) 
- Augmentation strategies should always be prescribed as an individual patient treatment trial, with review and discontinuation if any gains achieved fail to outweigh the side effects. (S)

\section{High-dose antipsychotic medication for treatment-resistant schizophrenia}

Howes et al. (2012b) found that before commencing clozapine, a third (34\%) of people with TRS had been prescribed high-dose antipsychotic medication. This relatively common use of high dosage to treat refractory schizophrenia suggests that clinicians have found it to be effective in a proportion of cases. However, the rationale for such a strategy is uncertain and the evidence base is thin. One justification would be low plasma drug levels leading to insufficient drug at the assumed effect site, dopamine D2 receptors. Low plasma drug levels might due to inadequate absorption, rapid metabolism and/or a contribution from smoking and genetic variation, for example, in cytochrome P450 enzymes (Horvitz-Lennon et al., 2017). But no clear evidence exists for a link between inadequate plasma antipsychotic medication levels and the effective use of high-dose antipsychotic medication for treatment-resistant illness. A more theoretical rationale would be that response to high-dose medication could reflect individual differences at the effect site. There may be some patients with high D2/3 receptor levels, perhaps due to the effects of prior antipsychotic treatment, who might require high doses to achieve sufficient blockade (Silvestri et al., 2000), but the data available suggest this is relatively rare (Howes et al., $2012 \mathrm{~b}$ ). There is no convincing evidence of a patient subgroup with higher dopamine D2 receptor levels or altered D2 receptor function. Indeed, compared with treatment-responsive schizophrenia, treatment-resistant illness seems to be characterised by a lack of dopaminergic abnormalities. Dopamine synthesis capacity is lower in treatment-refractory schizophrenia (and not different from healthy controls) than in those patients whose illnesses go on to show an adequate response to antipsychotic medication (Jauhar et al., 2019). Thus, there would seem to be an uncertain value in increasing the dose of a medication working via the same dopaminergic mechanism (Demjaha et al., 2012; Gillespie et al., 2017; Howes et al., 2009).

Analysis of the dose-response curves (derived from RCT data) for individual antipsychotic medications allowed Davis and Chen (2004) to calculate the 'near-maximal effective dose', a threshold dose necessary for all or almost all clinical response. The near-maximal effective dose was invariably below the top of the recommended range and there was no evidence that higher doses were more effective for schizophrenia generally or for treatment-resistant illness (Davis and Chen, 2004; Gardner et al., 2010). The Royal College of Psychiatrists' (2014) 'Consensus statement on high-dose antipsychotic medication' also concluded that for refractory schizophrenia there was no convincing evidence that antipsychotic dosage higher than the maximum licensed dose was more effective than standard dosage. The Canadian Agency for Drugs and Technologies in Health, Optimal Use Report (2011) recommended that high doses of a (non-clozapine) SGA should not be used instead of standard doses for TRS. Subsequent to these reports, the evidence base has been enhanced by meta-analyses of data from studies testing the efficacy of increasing antipsychotic dosage versus maintaining the same dose, in the treatment of schizophrenia responding poorly to an initial antipsychotic drug trial. Dold et al. (2015) examined five RCTs addressing this issue and found no differences between the dose-escalation and control groups in positive and negative symptom change scores, response or drop out. Samara et al. (2018) systematically reviewed nine, relevant, double-blind RCTs and one single-blind RCT. No clear differences were found between the increased dose and standard dose groups in change in PANSS total score, or the number of participants who either showed a clinically relevant response or who left the study early due to adverse effects. These investigators concluded that, in this clinical context, there was no good quality evidence to support or refute the hypothesis that increasing the dose of antipsychotic medication dose differed from continuing antipsychotic treatment at the same dose.

High-dose olanzapine for treatment-resistant schizophrenia. There is a long-standing notion that doses of olanzapine above the licensed maximum may be beneficial for TRS. Clinical trials have reported equivalent efficacy for high-dose olanzapine and standard doses of clozapine for such illness (Bitter et al., 2004; Conley, 1998; Meltzer et al., 2008; Tollefson et al., 2001) although clozapine proved to be superior in one 12-week, double-blind RCT (Kumra et al., 2008). Only a few double-blind RCTs have examined olanzapine in doses above $20 \mathrm{mg} /$ day, but reviewing the evidence, Citrome and Kantrowitz (2009) considered that such high dosage might be helpful in refractory illness characterised by severe, persistent symptoms and/or acute agitation. But they noted the need to balance any such benefit against an increased risk of metabolic side effects.

A systematic review and meta-analyses by Souza et al. (2013) included seven RCTs that had compared olanzapine with clozapine in patients with TRS. Clozapine was found to be superior to olanzapine for the treatment of positive and negative symptoms. However, there were no significant differences between the two medications on dropout rates or total PANSS scale score, although there was a trend towards high doses of olanzapine producing greater effect sizes. These investigators concluded that while the most robust evidence for efficacy in TRS favoured clozapine, the use of olanzapine, particularly in higher dosage, was a treatment option to be considered.

\section{Combined non-clozapine antipsychotic medications (antipsychotic polypharmacy) for treatment-resistant schizophrenia}

Like high-dose antipsychotic regimens, adding one or more antipsychotic medications to continuing antipsychotic treatment is a common and widespread clinical practice (Barnes and Paton, 2011; Hou et al., 2016; Kadra et al., 2016; Malandain et al., 2018; Sneider et al., 2015), especially for TRS (Thompson et al., 2016). This is despite a lack of robust evidence regarding both the efficacy and safety of such a strategy for illness that has proved to be unresponsive to standard antipsychotic monotherapy (Ballon and Stroup, 2013; Barnes and Paton, 2011; Gallego et al., 2012; Kadra et al., 2018).

Galling et al. (2017) conducted a systematic literature search and meta-analysis of RCTs that compared augmentation with a second antipsychotic with continued antipsychotic monotherapy in schizophrenia. While antipsychotic augmentation was found to be superior to monotherapy with regard to total symptom 
reduction in open-label and 'low-quality' trials, this was not the case for double-blind and 'high-quality' studies. Negative symptoms improved more with augmentation, but only in those studies augmenting with aripiprazole. A similar meta-analysis by the Cochrane Schizophrenia Group (Ortiz-Orendain et al., 2018) identified 62 relevant studies (total of 4833 participants), of which half compared clozapine monotherapy with a clozapine combination. Three-quarters of the studies were of short-term duration (shorter than 12 weeks). The findings included 'very low-quality' evidence that combined antipsychotic medication may improve clinical response and 'low-quality' evidence that, compared with monotherapy, such a strategy probably has little impact on relapse prevention or the emergence of serious adverse events. The main conclusion that may be drawn for the findings of these two metaanalyses is that there is a paucity of double-blind/high-quality evidence for symptom improvement and treatment response for augmentation of either clozapine or a non-clozapine antipsychotic medication with a second antipsychotic medication.

With regard to safety and tolerability, the use of combined antipsychotic medications is associated with a greater side-effect burden, specifically with regard to EPS, cognitive impairment, hyperprolactinaemia, sedation, sexual dysfunction, metabolic symptoms (Barnes and Paton, 2011; Centorrino et al., 2004; Fleischhacker and Uchida, 2014; Gallego et al., 2012) and diabetes (Kessing et al., 2010). Further, co-prescription of more than one antipsychotic is a risk factor for high-dose prescription (Barnes et al., 2009; Paton et al., 2008) and, as a more complex medication regimen, it may have a deleterious influence on adherence (Barnes and Paton, 2011).

In clinical practice, the implications of any perceived benefit with augmentation of antipsychotic monotherapy with a second antipsychotic for optimal long-term pharmacological treatment may be confounded. The symptomatic improvement may be simply attributable to more time on the first antipsychotic, related exclusively to the added antipsychotic medication, or due to a pharmacokinetic interaction and/or pharmacodynamic synergy between the two medications. In theory, this could be partly addressed by testing the efficacy of optimal use of each antipsychotic medication separately before prescribing the combination, but that rarely occurs in practice. But it may be erroneous to assume that any benefit seen is a direct consequence of the combination which should, therefore, be continued. This could lead to unnecessary antipsychotic polypharmacy and a high-dose antipsychotic prescription for an extended period.

In summary, the available evidence does not allow for any clear endorsement of antipsychotic polypharmacy in routine clinical practice. For schizophrenia which has failed to show an adequate response to several trials of standard antipsychotic monotherapy and where evidence-based treatments such as clozapine have been exhausted, guidelines tend to advocate that this should be conducted as a closely monitored, time-limited trial (Hasan et al., 2012; Langan and Shajahan, 2010; Miller, 2004).

\section{Recommendations for the management of incomplete recovery with high-dose or combined antipsychotic medication regimens}

- High-dose and combined antipsychotic prescribing for treatment-refractory schizophrenia should only be used after the failure of several, adequate, sequential trials of antipsychotic monotherapy, and other evidence-based treatments, including optimised treatment with clozapine, have been exhausted. (B)

- A high-dose or combined antipsychotic medication regimen should be regularly reviewed, with assessment of therapeutic response, including the target symptoms, and adverse effects. (S)

- Physical health monitoring for patients on a high-dose or combined antipsychotic medication regimen should include physical examination, haematological investigations and ECG. (C)

- The use of a high-dose or combined antipsychotic medication regimen for treatment-refractory schizophrenia should be treated as a limited therapeutic trial, with close monitoring of side effects and therapeutic response. The high dosage should be continued after 3 months only if there is evident clinical benefit that outweighs any risks. (S)

\section{Augmentation strategies for treatment- resistant schizophrenia with other classes of medication}

For those patients with schizophrenia whose illness has shown a sub-optimal response to antipsychotic medication, various augmentation strategies are used in clinical practice but none of these is supported by evidence that is reliable or robust enough to warrant it being considered as an established treatment of choice (Remington et al., 2017). Correll et al. (2017a) systematically and quantitatively compared the pharmacological combination strategies that have been studied for refractory schizophrenia. Specifically, they conducted a meta-analysis of RCTs testing the efficacy of add-on medication to antipsychotic treatment versus placebo or antipsychotic monotherapy. The studies examined involved 42 different psychotropic medications, including antidepressants, lamotrigine, selective serotonin 3 receptor antagonists (such as ondansetron and granisetron), oestrogen activity (sex hormones and oxytocin), minocycline, lithium, modafanil and topiramate. Of the 37 combination strategies with an antipsychotic medication other than clozapine that were tested, none had consistent supporting evidence for use in unselected patients. Of the five combinations strategies with clozapine that were tested, none showed superiority in terms of improvement in total psychopathology.

It is difficult to discern the implications for clinical practice of the findings of studies of augmentation strategies in people with a refractory schizophrenia. This is partly because of the lack of longer-term efficacy and safety data and the failure to address potential pharmacokinetic issues. More critically, many of these studies do not recognise the heterogeneity of clinical presentation of refractory schizophrenia. Clinicians will choose a particular augmentation medication to tackle particular key symptoms and behaviours that are persistent, have had a major adverse impact on the patient's social function and community re-integration, been a major cause of psychological distress and/or precluded discharge from hospital. But generally, patients have not been selected for participation in studies of particular augmentation regimens on the basis of the clinical indication targeted by such treatment in clinical practice. 
Augmentation with valproate for treatment-resistant schizophrenia. A Cochrane systematic review (Wang et al., 2016) concluded that there was limited evidence from open trials that valproate augmentation may be effective for overall clinical response in schizophrenia; any potential benefit is to be balanced against sedation and dizziness as common adverse effects. Tseng et al. (2016) conducted a meta-analysis of studies in people with schizophrenia or schizoaffective disorder that had compared valproate augmentation of antipsychotic medication with antipsychotic medication alone, with or without placebo. The main finding was that while valproate augmentation was associated with significantly greater improvement in symptoms in open trials this was not the case in RCTs. Further, the significant improvement was evident in short-term (less than 4 weeks' duration) studies but not in the longer-term studies (more than 4weeks). A larger meta-analysis examined studies of clozapine augmentation with topiramate, lamotrigine, sodium valproate or magnesium valproate versus clozapine alone (Zheng et al., 2017b). The five studies testing the addition of sodium valproate found significantly improved efficacy when this was assessed in terms of reduction in total PANSS score but not when study-defined response was applied as the outcome measure. Thus, at present there are insufficient data to support or refute the use of valproate as an effective adjunctive treatment for the core symptoms of schizophrenia.

Caution is advised if valproate is co-prescribed for clozapinetreated patients as such a combination is associated with an increased risk of neutropenia in such patients, both in those prescribed clozapine for the first time (Malik et al., 2018) and on rechallenge after a previous neutropenia (Meyer et al., 2015).

Augmentation with lamotrigine or topiramate for treatmentresistant schizophrenia. There is a lack of robust supportive evidence for a beneficial effect of either lamotrigine (Porcelli et al., 2012; Sommer et al., 2012; Vayisoğlu et al., 2013; Veerman et al., 2014) or topiramate (Behdani et al., 2011; Sommer et al., 2012; Veerman et al., 2014) as an adjunct to clozapine medication for schizophrenia that shown an insufficient response to clozapine treatment.

Adjunctive lithium for treatment-resistant schizophrenia. There is a lack of RCT evidence for lithium monotherapy as an effective treatment for schizophrenia (Leucht et al., 2015). Although early studies of adjunctive lithium for schizophrenia suggested that this might be a beneficial treatment, this was not supported by subsequent, better-designed studies (Citrome, 2009). A meta-analysis of 20 studies that tested lithium augmentation of antipsychotic medication against antipsychotic medication alone (Leucht et al., 2004) proved inconclusive. While more participants receiving lithium augmentation were classified as responders, this superiority was not consistent across different response thresholds. Further, when participants with prominent affective symptoms were omitted from the analysis, any significant advantage for lithium augmentation disappeared. Considering lithium augmentation of clozapine, a meta-analysis of relevant studies in people with TRS (Li et al., 2016) found no evidence of superiority for such a strategy over clozapine alone. Further, a 4-week, singleblind, randomised study in forensic patients with treatmentrefractory schizophrenia found no improvement with the addition of adjunctive lithium (Collins et al., 1991).

As Bender and Dittmann-Balcar (2004) point out, despite the limited evidence, schizophrenia treatment guidelines identify a range of indications for lithium, including adjunctive lithium for excitement, agitation and aggressive behaviour, and augmentation of clozapine for those patients with continuing prominent positive symptoms. However, whether a trial of lithium is worthwhile for a patient with a treatment-resistant illness, particularly when characterised by persistent affective symptoms and/or excitement, remains uncertain (Bender and Dittmann-Balcar, 2004; Citrome, 2009). Large-scale clinical trials in refractory schizophrenia are warranted, distinguishing between the effects of adjunctive lithium on affective symptoms and core schizophrenia features, such as positive and negative symptoms, as well systematically assessing the side-effect burden of such a regimen.

\section{Recommendations regarding pharmacological augmentation strategies for treatment-resistant schizophrenia}

- Given the lack of robust evidence for augmentation strategies, they should generally only be prescribed after evidencebased pharmacological strategies for treatment-resistant schizophrenia have been exhausted. (B)

- Augmentation strategies should be prescribed as individual treatment trials, being closely monitored for effectiveness for the target symptoms and/or behaviours as well as adverse effects. They should be continued long term only if there is evident benefit that outweighs any tolerability or safety concerns. (S)

\section{Declaration of Conflicting Interest}

The author(s) declared the following potential conflicts of interest with respect to the research, authorship, and/or publication of this article:

TREB: In the last 3 years, member of scientific advisory boards for Lundbeck, Newron Pharmaceuticals and Gedeon Richter and in receipt of speaker fees from Janssen.

RD: No personal interests to declare.

CP: No personal interests to declare.

SJC: From 2010 to 2018, Clinical Lead for the National Audit of Schizophrenia/National Clinical Audit of Psychosis. No fees, grants or honoraria from pharmaceutical companies accepted during the last 10 years but travel expenses accepted, where necessary, to present NAS/ NCAP findings.

BD: In the last 3 years, BD has carried out experimental studies in healthy volunteers funded by Autifony, Sunovion and P1vital. He is a paid member of the scientific advisory board of Autifony.

INF: No personal interests to declare.

CJG: No personal interests to declare.

PMH: In the last 3 years, PMH has received fees for lecturing and/or consultancy work (including attending advisory boards) from Janssen, Lundbeck, NewBridge Pharmaceuticals, Otsuka and Sunovion and conference support from Sunovion.

ODH: Investigator-initiated research funding from and/or participated in advisory/speaker meetings organised by Angellini, Autifony, Biogen, Heptares, Jansenn, Lundbeck-Otsuka, Lyden-Delta, Sunovion, and Roche. Neither ODH nor his family have been employed by or have holdings/a financial stake in any pharmaceutical company.

IJ: No personal interests to declare.

EMJ: No personal interests to declare.

SL: In the last 3 years, CDAs with J\&J, Otsuka Health Sciences. Founding Director, Affigo CiC. Medical director, Xenzone PLC.

ALH: In the last 3 years, ALH has received fees for lecturing and/ or consultancy work Lundbeck, Janssen-Cilag, Silence and unpaid 
consultancy with Opiant, Dobrin, Lightlake, Britannia, GLG.

JHM: In the last 3 years, JHM has received travel and accommodation expenses and research funding from Lundbeck.

DCO: No personal interests to declare.

MXP: In the last 3 years, MXP has been a member of scientific advisory boards for Janssen and Lundbeck and has been PI on studies sponsored by Takeda and Amgen.

JMAS: No personal interests to declare.

JMS: In the last 3 years, JMS has received fees for consultancy work from Janssen Pharmaceutica and has been PI or sub-investigator on studies sponsored by Takeda, Janssen, Lundbeck and Allergan Plc. PST: Between 2015 and 2018 PST received consultancy fees as an Advisory Board member for Galen Limited; Sunovion Pharmaceuticals Europe Ltd; myTomorrows; and LivaNova UK Ltd. Since 2018 PTS has been sub-investigator on a clinical drug trial sponsored by COMPASS Pathways Ltd, UK.

RU: In the last 3 years, fees received for lecturing from Lundbeck.

AW: No personal interests to declare.

ARY: In the last 3 years, fees received for lecturing and/or consultancy work and conference support from Janssen and Boehringer-Ingelheim.

\section{Funding}

The author(s) received no financial support for the research, authorship, and/or publication of this article.

\section{ORCID iDs}

Thomas R E Barnes iD https://orcid.org/0000-0002-2324-656X

Carol Paton (iD) https://orcid.org/0000-0001-7756-1031

James M Stone (iD https://orcid.org/0000-0003-3051-0135

\section{References}

Abdel-Baki A, Ouellet-Plamondon C and Malla A (2012) Pharmacotherapy challenges in patients with first-episode psychosis. $J$ Affect Disord 138(Suppl): S3-S14.

Addington D, Addington J and Maticka TE (1993) Assessing depression in schizophrenia: The Calgary Depression Scale. Br J Psychiatry Suppl 22: 39-44.

Addington J and Duchak V (1997) Reasons for substance use in schizophrenia. Acta Psychiatr Scand 96: 329-333.

Adeosun II (2017) Are depot antipsychotics associated with longer persistence in treatment compared with oral antipsychotics among patients with schizophrenia? J Pharm Res Int 18: 1-8.

Agid O, Arenovich T, Sajeev G, et al. (2011) An algorithm-based approach to first-episode schizophrenia: Response rates over 3 prospective antipsychotic trials with a retrospective data analysis. $J$ Clin Psychiatry 72: 1439-1444.

Agid O, Kapur S, Arenovich T, et al. (2003) Delayed-onset hypothesis of antipsychotic action: A hypothesis tested and rejected. Arch Gen Psychiatry 60: 1228-1235.

Agid O, Schulze L, Arenovich T, et al. (2013) Antipsychotic response in first-episode schizophrenia: Efficacy of high doses and switching. Eur Neuropsychopharmacol 23: 1017-1022.

Agid O, Seeman P and Kapur S (2006) The "delayed onset" of antipsychotic action - an idea whose time has come and gone. J Psychiatry Neurosci 31: 93-100.

Ahmed S, Chengappa KN, Naidu VR, et al. (1998) Clozapine withdrawal-emergent dystonias and dyskinesias: A case series. J Clin Psychiatry 59: 472-477.

Akdede BB, Anil Yağcioğlu AE, Alptekin K, et al. (2006) A doubleblind study of combination of clozapine with risperidone in patients with schizophrenia: Effects on cognition. J Clin Psychiatry 67: 1912-1919.
Alaqeel B and Margolese HC (2013) Remission in schizophrenia: Critical and systematic review. Harv Rev Psychiatry 20: 281-297.

Albon E, Tsourapas A, Frew E, et al. (2008) Structural neuroimaging in psychosis: A systematic review and economic evaluation. Health Technol Assess 12: iii-iv, ix-163.

Al-Diwani A, Handel A, Townsend L, et al. (2019) The psychopathology of NMDAR-antibody encephalitis in adults: A systematic review and phenotypic analysis of individual patient data. Lancet Psychiatry 6: 235-246.

Almerie MQ, El-Din Matar H, Essali A, et al. (2008) Cessation of medication for people with schizophrenia already stable on chlorpromazine. Schizophr Bull 34: 13-14.

Alphs L, Nasrallah HA, Bossie CA, et al. (2016) Factors associated with relapse in schizophrenia despite adherence to long-acting injectable antipsychotic therapy. Int Clin Psychopharmacol 31: 202-209.

Altshuler LL, Cohen L, Szuba MP, et al. (1996) Pharmacologic management of psychiatric illness during pregnancy: Dilemmas and guidelines. Am J Psychiatry 15: 592-606.

Alvarez-Jimenez M, O’Donoghue B, Thompson A, et al. (2016) Beyond clinical remission in first episode psychosis: Thoughts on antipsychotic maintenance vs. guided discontinuation in the functional recovery era. CNS Drugs 30: 357-368.

Alvarez-Jimenez M, Priede A, Hetrick SE, et al. (2012) Risk factors for relapse following treatment for first episode psychosis: A systematic review and meta-analysis of longitudinal studies. Schizophr Res 139: $116-128$.

Al-Zakwani IS, Barron JJ, Bullano MF, et al. (2003) Analysis of healthcare utilization patterns and adherence in patients receiving typical and atypical antipsychotic medications. Curr Med Res Opin 19: 619-626.

American Psychiatric Association (1994) Diagnostic and Statistical Manual of Mental Disorders: DSM-IV. Arlington: American Psychiatric Pub. Inc.

American Psychiatric Association (2013) Diagnostic and Statistical Manual of Mental Disorders. 5th ed. Arlington: American Psychiatric Association.

Amminger G, Schäfer MR, Papageorgiou K, et al. (2010) Long-chain $\omega-3$ fatty acids for indicated prevention of psychotic disorders: A randomized, placebo-controlled trial. Arch Gen Psychiatry 67: 146154.

Amminger GP, Schäfer MR, Schlögelhofer M, et al. (2015) Longer-term outcome in the prevention of psychotic disorders by the Vienna omega-3 study. Nat Commun 6: 7934.

Anderson KE, Stamler D, David MD, et al. (2017) Deutetrabenazine for treatment of involuntary movements in patients with tardive dyskinesia (AIM-TD): A double-blind, randomised, placebo-controlled, phase 3 trial. Lancet Psychiatry 4: 595-604.

Andreasen NC (1989) The Scale for the Assessment of Negative Symptoms (SANS): Conceptual and theoretical foundations. Br J Psychiatry Suppl 7: 49-58.

Andreasen NC, Carpenter WT Jr, Kane JM, et al. (2005) Remission in schizophrenia: Proposed criteria and rationale for consensus. $\mathrm{Am} \mathrm{J}$ Psychiatry 162: 441-449.

Anil Yağcioğlu AE, Kivircik Akdede BB, Turgut TI, et al. (2005) A double-blind controlled study of adjunctive treatment with risperidone in schizophrenic patients partially responsive to clozapine: Efficacy and safety. J Clin Psychiatry 66: 63-72.

Anthenelli RM, Benowitz NL, West R, et al. (2016) Neuropsychiatric safety and efficacy of varenicline, bupropion, and nicotine patch in smokers with and without psychiatric disorders (EAGLES): A double-blind, randomised, placebo-controlled clinical trial. Lancet 387: 2507-2520.

Arango C, Bombin I, Gonzalez-Salvador T, et al. (2006) Randomised clinical trial comparing oral versus depot formulations of zuclopenthixol in patients with schizophrenia and prevous violence. Eur Psychiatry 21: 34-40. 
Ascher-Svanum H, Faries DE, Zhu B, et al. (2006) Medication adherence and long-term functional outcomes in the treatment of schizophrenia in usual care. J Clin Psychiatry 67: 453-460.

Asenjo Lobos C, Komossa K, Rummel-Kluge C, et al. (2010) Clozapine versus other atypical antipsychotics for schizophrenia. Cochrane Database Syst Rev 11: CD006633.

Assion HJ, Reinbold H, Lemanski S, et al. (2008) Amisulpride augmentation in patients with schizophrenia partially responsive or unresponsive to clozapine. A randomized, double-blind, placebo-controlled trial. Pharmacopsychiatry 41: 24-28.

Aston J, Rechsteiner E, Bull N, et al. (2010) Hyperprolactinaemia in early psychosis - not only due to antipsychotics. Prog Neuropsychopharmacol Biol Psychiatry 34: 1342-1344.

Atakan Z, Cliff G and Cooper JE (1990) Can psychiatrists predict the one-year outcome of schizophrenia? Soc Psychiatry Psychiatr Epidemiol 25: 117-124.

Atkins S, Detke HC, McDonnell DP, et al. (2014) A pooled analysis of injection site-related adverse events in patients with schizophrenia treated with olanzapine long-acting injection. BMC Psychiatry 14: 7.

Ayesa-Arriola R, Alcaraz EG, Hernández BV, et al. (2015) Suicidal behaviour in first-episode non-affective psychosis: Specific risk periods and stage-related factors. Eur Neuropsychopharmacol 25: $2278-2288$

Baandrup L, Gasse C, Jensen VD, et al. (2010) Antipsychotic polypharmacy and risk of death from natural causes in patients with schizophrenia: A population-based nested case-control study. J Clin Psychiatry 71: 103-108.

Baldwin D and Wieck A (2018) Withdrawal of, and alternatives to, valproate-containing medicines in girls and women of childbearing potential who have a psychiatric illness. Royal College of Psychiatrists Position Statement PS04/18.

Ballon J and Stroup TS (2013) Polypharmacy for schizophrenia. Curr Opin Psychiatry 26: 208.

Ban L, Fleming KM, Doyle P, et al. (2015) Congenital anomalies in children of mothers taking antiepileptic drugs with and without periconceptional high dose folic acid use: A population-based cohort study. PLoS One 10: e0131130.

Barber S, Olotu U, Corsi M, et al. (2017) Clozapine combined with different antipsychotic drugs for treatment-resistant schizophrenia. Cochrane Database Syst Rev 3: CD006324.

Barbui C, Saraceno B, Liberati A, et al. (1996) Low-dose neuroleptic therapy and relapse in schizophrenia: Meta-analysis of randomized controlled trials. Eur Psychiatry 11: 306-313.

Barbui C, Signoretti A, Mulè S, et al. (2009) Does the addition of a second antipsychotic drug improve clozapine treatment? Schizophr Bull 35: 458-468.

Barch DM, Marder SR, Harms MP, et al. (2016) Task-related fMRI responses to a nicotinic acetylcholine receptor partial agonist in schizophrenia: A randomized trial. Prog Neuropsychopharmacol Biol Psychiatry 71: 66-75.

Barkhof E, Meijer CJ, de Sonneville LM, et al. (2013) The effect of motivational interviewing on medication adherence and hospitalization rates in nonadherent patients with multi-episode schizophrenia. Schizophr Bull 39: 1242-1251.

Barnes TR; The Schizophrenia Consensus Group of the British Association for Psychopharmacology (2011) Evidence-based guidelines for the pharmacological treatment of schizophrenia: Recommendations from the British Association for Psychopharmacology. J Psychopharmacol 25: 567-620.

Barnes TRE (1987) The present status of tardive dyskinesia and akathisia in the treatment of schizophrenia. Psychiatric Dev 4: 301-319.

Barnes TRE (2005) Why indeed? Invited commentary on: Why aren't depot antipsychotics prescribed more often and what can be done about it? Adv Psychiatr Treat 11: 211-213.

Barnes TRE, Bhatti SF, Adroer R, et al. (2015) Screening for the metabolic side effects of antipsychotic medication: Findings from a 6-year quality improvement programme in the UK. BMJ Open 5: e007633.

Barnes TRE and Braude WM (1985) Akathisia variants and tardive dyskinesia. Arch Gen Psychiatry 42: 874-878.

Barnes TRE and Curson DA (1994) Long-acting depot antipsychotics: A risk-benefit assessment. Drug Safety 10: 464-479.

Barnes TRE, Curson DA, Liddle PF, et al. (1989) The nature and prevalence of depression in chronic schizophrenic in-patients. Br J Psychiatry 154: 486-491.

Barnes TRE, Drake RJ, Dunn G, et al. (2013) Effect of prior treatment with antipsychotic long-acting injection on randomised clinical trial treatment outcomes. Br J Psychiatry 203: 215-220.

Barnes TRE and Kerwin R (2003) Mortality and sudden death in schizophrenia. In: J. Camm (eds) Cardiovascular Risk Associated with Schizophrenia and its Treatment. London: Galliard Healthcare Communications, 7-23.

Barnes TRE, Leeson V, Paton C, et al. (2018) Amisulpride augmentation of clozapine for treatment-refractory schizophrenia: A doubleblind, placebo-controlled trial. Ther Adv Psychopharmacol 8: 185197.

Barnes TRE, Leeson VC, Paton C, et al. (2016) Antidepressant controlled trial for negative symptoms in schizophrenia (ACTIONS): A doubleblind, placebo-controlled, randomised clinical trial. Health Technol Assess 20: 1-46.

Barnes TR and Paton C (2011) Antipsychotic polypharmacy in schizophrenia: Benefits and risks. CNS Drugs 25: 383-399.

Barnes TRE, Shingleton-Smith A and Paton C (2009) Treatment of schizophrenia by long-acting depot injections in the UK. Br J Psychiatry 195(Suppl): s37-s42.

Beach SR, Celano CM, Sugrue AM, et al. (2018) QT prolongation, Torsades de Pointes, and psychotropic medications: A 5-year update. Psychosomatics 59: 105-122.

Bechdolf A, Müller H, Stützer H, et al. (2016) PREVENT: A randomized controlled trial for the prevention of first episode psychosis comparing cognitive-behavior therapy (CBT), clinical management, and aripiprazole combined and clinical management and placebo combined. Schizophr Bull 43: S56.

Beck K, McCutcheon R, Bloomfield MA, et al. (2014) The practical management of refractory schizophrenia - the Maudsley Treatment Review and Assessment Team service approach. Acta Psychiatr Scand 130: 427-438.

Behdani F, Hebrani P, Rezaei Ardani A, et al. (2011) Effect of topiramate augmentation in chronic schizophrenia: A placebo-controlled trial. Arch Iran Med 14: 270-275.

Beinat TC, Banister SD, Herrera M, et al. (2015) The therapeutic potential of alpha7 nicotinic acetylcholine receptor (alpha7 nAChR) agonists for the treatment of the cognitive deficits associated with schizophrenia. CNS Drugs 29: 529-542.

Bender S and Dittmann-Balcar A (2004) Review: In people with schizophrenia, lithium is ineffective as sole therapy, while evidence on augmenting antipsychotics with lithium is inconclusive. Evid Based Ment Health 7: 104.

Bennett PN (1996) Use of the monographs on drugs. In: Bennett PN (ed.) Drugs and Human Lactation. Amsterdam: Elsevier Science Publishers, 67-74.

Bergman H, Walker D-M, Nikolakopoulou A, et al. (2017) Systematic review of interventions for treating or preventing antipsychoticinduced tardive dyskinesia. Health Technol Assess 21: 1-218.

Berk M, Copolov D, Dean O, et al. (2008) N-acetyl cysteine as a glutathione precursor for schizophrenia-a double-blind, randomized, placebo-controlled trial. Biol Psychiatry 64: 361-368.

Berk M, Fitzsimons J, Lambert T, et al. (2007) Monitoring the safe use of clozapine: A consensus view from Victoria, Australia. CNS Drugs 21: 117-127.

Berwaerts J, Liu Y, Gopal S, Nuamah I, et al. (2015) Efficacy and safety of the 3-month formulation of paliperidone palmitate vs placebo for 
relapse prevention of schizophrenia: A randomized clinical trial. JAMA Psychiatry 72: 830-839.

Bhakta SG, Chou HH, Rana B, et al. (2016) Effects of acute memantine administration on MATRICS Consensus Cognitive Battery performance in psychosis: Testing an experimental medicine strategy. Psychopharmacology (Berl) 233: 2399-2410.

Bhavsar V, Kosidou K, Widman L, et al. (2019) Clozapine treatment and offending: A within-subject study of patients with psychotic disorders in Sweden. Schizophr Bull. Epub ahead of print 31 May 2019. doi:10.1093/schbul/sbz055.

Bilder RM, Goldman RS, Volavka J, et al. (2002) Neurocognitive effects of clozapine, olanzapine, risperidone, and haloperidol in patients with chronic schizophrenia or schizoaffective disorder. Am J Psychiatry 159: 1018-1028.

Birchwood M, Iqbal Z and Upthegrove R (2005) Psychological pathways to depression in schizophrenia: Studies in acute psychosis, post psychotic depression and auditory hallucinations. Eur Arch Psychiatry Clin Neurosci 255: 202-212.

Birchwood M, Spencer E and McGovern D (2000) Schizophrenia: Early warning signs. Adv Psychiatr Treat 6: 93-101.

Bitter I, Dossenbach MR, Brook S, et al. (2004) Olanzapine versus clozapine in treatment-resistant or treatment-intolerant schizophrenia. Prog Neuropsychopharmacol Biol Psychiatry 28: 173-180.

Bitter I, Treuer T, Dyachkova Y, et al. (2008) Antipsychotic prescription patterns in outpatient settings: 24-month results from the Intercontinental Schizophrenia Outpatient Health Outcomes (IC-SOHO) study. Eur Neuropsychopharmacol 18: 170-180.

Blackburn R, Osborn D, Walters K, et al. (2017) Statin prescribing for people with severe mental illnesses: A staggered cohort study of 'real-world' impacts. BMJ Open 7: e013154.

Bloch Y, Mendlovic S, Strupinsky S, et al. (2001) Injections of depot antipsychotic medications in patients suffering from schizophrenia: Do they hurt? J Clin Psychiatry 62: 855-859.

Blom MT, Cohen D, Seldenrijk A, et al. (2014) Brugada syndrome ECG is highly prevalent in schizophrenia. Circ Arrhythm Electrophysiol 7: 384-391.

Bola JR, Lehtinen K, Aaltonen J, et al. (2006) Predicting medicationfree treatment response in acute psychosis: Cross-validation from the Finnish Need-Adapted Project. J Nerv Ment Dis 194: 732-739.

Bola JR and Mosher LR (2003) Treatment of acute psychosis without neuroleptics: Two-year outcomes from the Soteria project. $J$ Nerv Ment Dis 191: 219-229

Boonstra N, Klaassen R, Sytema S, et al. (2012) Duration of untreated psychosis and negative symptoms - a systematic review and meta-analysis of individual patient data. Schizophr Res 142: $12-19$.

Boucher N, Bairam A and Beaulac-Baillargeon L (2008) A new look at the neonate's clinical presentation after in utero exposure to antidepressants in late pregnancy. J Clin Psychopharmacol 28: 334-339.

Bowen J and Barnes TRE (1994) The clinical characteristics of schizophrenic patients consenting and not consenting to a placebo-controlled trial. Human Psychopharmacol 9: 423-433.

Bowskill S, Couchman L, MacCabe JH, et al. (2012) Plasma clozapine and norclozapine in relation to prescribed dose and other factors in patients aged 65 years and over: Data from a therapeutic drug monitoring service, 1996-2010. Hum Psychopharmacol 27: 277-283.

Bowtell M, Ratheesh A, McGorry P, et al. (2018) Clinical and demographic predictors of continuing remission or relapse following discontinuation of antipsychotic medication after a first episode of psychosis. A systematic review. Schizophr Res 197: 9-18.

Brain C, Allerby K, Sameby B, et al. (2013) Drug attitude and other predictors of medication adherence in schizophrenia: 12 months of electronic monitoring (MEMS( $\left({ }^{2}\right)$ ) in the Swedish COAST-study. Eur Neuropsychopharmacol 23: 1754-1762.
Brain C, Sameby B, Allerby K, et al. (2014) Twelve months of electronic monitoring (MEMS ${ }^{\circledR}$ ) in the Swedish COAST-study: A comparison of methods for the measurement of adherence in schizophrenia. Eur Neuropsychopharmacol 24: 215-222.

Branchey M and Branchey L (1984) Patterns of psychotropic drug use and tardive dyskinesia. J Clin Psychopharmacol 4: 41-45.

Braude WM, Barnes TRE and Gore SM (1983) Clinical characteristics of akathisia. A systematic investigation of acute psychiatric inpatient admissions. Br J Psychiatry 143: 139-150.

Brauer R, Smeeth L, Anaya-Izquierdo K, et al. (2015) Antipsychotic drugs and risks of myocardial infarction: A self-controlled case series study. Eur Heart J 36: 984-992.

Breier A, Liffick E, Hummer TA, et al. (2018) Effects of 12-month, double-blind $\mathrm{N}$-acetyl cysteine on symptoms, cognition and brain morphology in early phase schizophrenia spectrum disorders. Schizophr Res 199: 395-402.

Brekke JS, Prindle C, Bae SW, et al. (2001) Risks for individuals with schizophrenia who are living in the community. Psychiatr Serv 52: 1358-1366.

British National Formulary. National Institute for Health and Care Excellence. Available at: https://bnf.nice.org.uk/ (accessed 30 September 2019).

Broome MR, Woolley JB, Johns LC, et al. (2005) Outreach and support in south London (OASIS): Implementation of a clinical service for prodromal psychosis and the at risk mental state. Eur Psychiatry 20: 372-378.

Brown S, Kim M, Mitchell C, et al. (2010) Twenty-five year mortality of a community cohort with schizophrenia. Br J Psychiatry 196: $116-121$.

Buchanan RW, Conley RR, Dickinson D, et al. (2008) Galantamine for the treatment of cognitive impairments in people with schizophrenia. Am J Psychiatry 165: 82-89.

Buchanan RW, Javitt DC, Marder SR, et al. (2007) The Cognitive and Negative Symptoms in Schizophrenia Trial (CONSIST): The efficacy of glutamatergic agents for negative symptoms and cognitive impairments. Am J Psychiatry 164: 1593-1602.

Buchanan RW, Kelly DL, Weiner E, et al. (2017) A randomized clinical trial of oxytocin or galantamine for the treatment of negative symptoms and cognitive impairments in people with schizophrenia. J Clin Psychopharmacol 37: 394-400.

Buchanan RW, Kreyenbuhl J, Kelly DL, et al. (2010) The 2009 schizophrenia PORT psychopharmacological treatment recommendations and summary statements. Schizophr Bull 36: 71-93.

Buckley P, Bartell J, Donenwirth K, et al. (1995) Violence and schizophrenia: Clozapine as a specific antiaggressive agent. Bull Am Acad Psychiatry Law 23: 607-611.

Buckley PF, Miller BJ, Lehrer DS, et al. (2009) Psychiatric comorbidities and schizophrenia. Schizophr Bull 35: 383-402.

Buckley NA and Sanders P (2000) Cardiovascular adverse effects of antipsychotic drugs. Drug Saf 23: 215-228.

Bugarski-Kirola D, Blaettler T, Arango C, et al. (2017) Bitopertin in negative symptoms of schizophrenia results from the phase III FlashLyte and DayLyte studies. Biol Psychiatry 82: 8-16.

Buhler B, Hambrecht M, Loffler W, et al. (2002) Precipitation and determination of the onset and course of schizophrenia by substance abuse: Retrospective and prospective study of 232 population-based first illness episodes. Schizophr Res 54: 243-251.

Burkhard PR (2014) Acute and subacute drug-induced movement disorders. Parkinsonism Relat Disord 20(Suppl 1): S108-S112.

Byerly MJ, Fisher R, Carmody T, et al. (2005) A trial of compliance therapy in outpatients with schizophrenia or schizoaffective disorder. J Clin Psychiatry 66: 997-1001.

Byerly MJ, Nakonezny PA, Bettcher BM, et al. (2006) Sexual dysfunction associated with second-generation antipsychotics in outpatients with schizophrenia or schizoaffective disorder: An empirical evaluation of olanzapine, risperidone, and quetiapine. Schizophr Res 86: 244-250. 
Byerly MJ, Nakonezny PA and Lescouflair E (2007) Antipsychotic medication adherence in schizophrenia. Psychiatr Clin North Am 30: 437-452.

Byerly MJ, Nakonezny PA and Rush AJ (2008) The Brief Adherence Rating Scale (BARS) validated against electronic monitoring in assessing the antipsychotic medication adherence of outpatients with schizophrenia and schizoaffective disorder. Schizophr Res 100: 60-69.

Cadenhead K, Addington J, Cannon T, et al. (2017) Omega-3 fatty acid versus placebo in a clinical high-risk sample from the North American Prodrome Longitudinal studies (NAPLS) consortium. Schizophr Bull 43: S16.

Cahill K, Lindson-Hawley N, Thomas KH, et al. (2016) Nicotine receptor partial agonists for smoking cessation. Cochrane Database Syst Rev 5: CD006103.

Cain CK, McCue M, Bello I, et al. (2014) d-Cycloserine augmentation of cognitive remediation in schizophrenia. Schizophr Res 153: 177-183.

Calton T, Ferriter M, Huband M, et al. (2008) A systematic review of the Soteria paradigm for the treatment of people diagnosed with schizophrenia. Schizophr Bull 34: 181-192.

Canadian Agency for Drugs and Technologies in Health (2011) Optimal use recommendations for atypical antipsychotics: Combination and high-dose treatment strategies in adolescents and adults with schizophrenia. CADTH Optimal Use Report, No. 1.1C. Ottawa (ON): Canadian Agency for Drugs and Technologies in Health.

Canuso CM, Schooler N, Carothers J, et al. (2010) Paliperidone extendedrelease in schizoaffective disorder: A randomized, controlled study comparing a flexible dose with placebo in patients treated with and without antidepressants and/or mood stabilisers. J Clin Psychopharmacol 30: 487-495.

Carbon M, Hsieh CH, Kane JM, et al. (2017) Tardive dyskinesia prevalence in the period of second-generation antipsychotic use: A metaanalysis. J Clin Psychiatry 78: e264-e278.

Carbon M, Kane JM, Leucht S, et al. (2018) Tardive dyskinesia risk with first- and second-generation antipsychotics in comparative randomized controlled trials: A meta-analysis. World Psychiatry 17: 330-340.

Carney R, Cotter J, Firth J, et al. (2016) Cardiometabolic risk factors in young people at ultra-high risk for psychosis: A systematic review and meta-analysis. Schizophr Res 170: 290-300.

Carney R, Cotter J, Firth J, et al. (2017) Cannabis use and symptom severity in individuals at ultra high risk for psychosis: A meta-analysis. Acta Psychiatr Scand 136: 5-15.

Carpenter WT and Buchanan RW (2008) Lessons to take home from CATIE. Psychiatr Serv 59: 523-525.

Carpenter WT, Jr (2001) Evidence-based treatment for first-episode schizophrenia? Am J Psychiatry 158: 1771-1773.

Carpenter WT, Hanlon TE, Heinrichs DW, et al. (1990) Continuous versus targeted medication in schizophrenic outpatients: Outcome results. Am J Psychiatry 147: 1138-1148.

Castle DJ, Galletly CA, Dark F, et al. (2017) The 2016 Royal Australian and New Zealand College of Psychiatrists guidelines for the management of schizophrenia and related disorders. Med J Aust 206: 501-505.

Caton CL, Hasin DS, Shrout PE, et al. (2007) Stability of early phase primary psychotic disorders with concurrent substance use and substance-induced psychosis. Br J Psychiatry 190: 105-111.

Centorrino F, Goren JL, Hennen J, et al. (2004) Multiple versus single antipsychotic agents for hospitalized psychiatric patients: casecontrol study of risks and benefits. Am J Psychiatry 161: 700-706.

Chakos M, Lieberman J, Hoffman E, et al. (2001) Effectiveness of second-generation antipsychotics in patients with treatment-resistant schizophrenia: A review and meta-analysis of randomized trials. $\mathrm{Am}$ J Psychiatry 158: 518-526.

Chakos MH, Alvir JM, Woerner MG, et al. (1996) Incidence and correlates of tardive dyskinesia in first episode of schizophrenia. Arch Gen Psychiatry 53: 313-319.
Chakos MH, Mayerhoff DI, Loebel AD, et al. (1992) Incidence and correlates of acute extrapyramidal symptoms in first episode schizophrenia. Psychopharmacol Bull 28: 81-86.

Chang JS, Ahn YM, Park HJ, et al. (2008) Aripiprazole augmentation in clozapine-treated patients with refractory schizophrenia: An 8-week, randomized, double-blind, placebo-controlled trial. J Clin Psychiatry 69: 720-731.

Chen AT, Chibnall JT and Nasrallah HA (2015) A meta-analysis of placebo-controlled trials of omega-3 fatty acid augmentation in schizophrenia: Possible stage-specific effects. Ann Clin Psychiatry 27: 289-296.

Chen EYH, Hui CLM, Lam MML, et al. (2010) Maintenance treatment with quetiapine versus discontinuation after one year of treatment in patients with remitted first episode psychosis: Randomised controlled trial. BMJ 341: c4024.

Chengappa KN, Turkin SR, Desanti S, et al. (2012) A preliminary, randomized, double-blind, placebo-controlled trial of L-carnosine to improve cognition in schizophrenia. Schizophr Res 142: 145-152.

Chien WT, Cheung EF, Mui JH, et al. (2019) Adherence therapy for schizophrenia: A randomised controlled trial. Hong Kong Med J 25(Suppl 2): 4-9.

Chiliza B, Ojagbemi A, Esan O, et al. (2016) Combining depot antipsychotic with an assertive monitoring programme for treating firstepisode schizophrenia in a resource-constrained setting. Early Interv Psychiatry 10: 54-62.

Cho J, Hayes RD, Jewell A, et al. (2019a) Clozapine and all-cause mortality in treatment-resistant schizophrenia: A historical cohort study. Acta Psychiatr Scand 139: 237-247.

Cho M, Lee TY, Kwak YB, et al. (2019b) Adjunctive use of anti-inflammatory drugs for schizophrenia: A meta-analytic investigation of randomized controlled trials. Aust N Z J Psychiatry 53: 742-759.

Chong SA, Remington GJ and Bezchlibnyk-Butler KZ (2000) Effect of clozapine on polypharmacy. Psychiatr Serv 51: 250-252.

Chong SA, Tan CH and Lee HS (1997) Atrial ectopics with clozapinerisperidone combination. J Clin Psychopharmacol 17: 130-131.

Choong LS, Coupe T and Vijaya JP (2011) A simple clinical audit of antipsychotic medication prescribing on Hollywell Psychiatric Intensive Care Unit (PICU). J Psychiatr Intensive Care 8: 11-14.

Chouinard G (1991) Severe cases of neuroleptic-induced supersensitivity psychosis. Diagnostic criteria for the disorder and its treatment. Schizophr Res 5: 21-33.

Chouinard G, Samaha AN, Chouinard VA, et al. (2017) Antipsychoticinduced dopamine supersensitivity psychosis: Pharmacology, criteria, and therapy. Psychother Psychosom 86: 189-219.

Christensen J, Gronborg TK, Sorensen MJ, et al. (2013) Prenatal valproate exposure and risk of autism spectrum disorders and childhood autism. JAMA 309: 1696-1703.

Chuang YF, Elango P, Gonzalez CE, et al. (2017) Midlife anticholinergic drug use, risk of Alzheimer's disease and brain atrophy in community-dwelling older adults. Alzheimer's Dement (NY) 3: 471-479.

Citrome L (2009) Adjunctive lithium and anticonvulsants for the treatment of schizophrenia: What is the evidence? Expert Rev Neurother 9: 55-71.

Citrome L (2013) New second-generation long-acting injectable antipsychotics for the treatment of schizophrenia. Expert Rev Neurother 13: 767-783.

Citrome L and Kantrowitz JT (2009) Olanzapine dosing above the licensed range is more efficacious than lower doses: Fact or fiction? Expert Rev Neurother 9: 1045-1058.

Citrome L, McEvoy JP and Saklad SR (2016) Guide to the management of clozapine-related tolerability and safety concerns. Clin Schizophr Relat Psychoses 10: 163-177.

Citrome L, Shope CB, Nolan KA, et al. (2007) Risperidone alone versus risperidone plus valproate in the treatment of patients with schizophrenia and hostility. Int Clin Psychopharmacol 22: 356-362. 
Citrome L and Volavka J (2011) Pharmacological management of acute and persistent aggression in forensic psychiatry settings. CNS Drugs 25: 1009-1021.

Citrome L and Volavka J (2014) The psychopharmacology of violence: Making sensible decisions. CNS Spect 19: 411-418.

Citrome L, Volavka J, Czobor P, et al. (2001) Effects of clozapine, olanzapine, risperidone, and haloperidol on hostility among patients with schizophrenia. Psychiatr Serv 52: 1510-1514.

Ciudad A, Haro JM, Alonso J, et al. (2008) The Schizophrenia Outpatient Health Outcomes (SOHO) study: 3-year results of antipsychotic treatment discontinuation and related clinical factors in Spain. Eur Psychiatry 23: 1-7.

Claassen D (2007) Financial incentives for antipsychotic depot medication: Ethical issues. $J$ Med Ethics 33: 189-193.

Clayton-Smith J, Bromley R, Dean J, et al. (2019) Diagnosis and management of individuals with fetal valproate spectrum disorder; a consensus statement from the European Reference Network for Congenital Malformations and Intellectual Disability. Orphanet J Rare Dis 14: 180.

Cleare A, Pariante CM, Young AH, et al. (2015) Evidence-based guidelines for treating depressive disorders with antidepressants: A revision of the 2008 British Association for Psychopharmacology guidelines. J Psychopharmacol 29: 459-525.

Clozaril, Summary of product characteristics. Available at: www. medicines.org.uk (accessed September 2019).

Coldham EL, Addington J and Addington D (2002) Medication adherence of individuals with a first episode of psychosis. Acta Psychiatr Scand 106: 286-290.

Coleman CI, Limone B, Sobieraj DM, et al. (2012) Dosing frequency and medication adherence in chronic disease. J Manag Care Pharm 18: 527-539.

Collins PJ, Larkin EP and Shubsachs AP (1991) Lithium carbonate in chronic schizophrenia - a brief trial of lithium carbonate added to neuroleptics for treatment of resistant schizophrenic patients. Acta Psychiatr Scand 84: 150-154.

Conley RR (1998) Optimising treatment with clozapine. J Clin Psychiatry 59(Suppl 3): 44-48.

Conley RR, Ascher-Svanum H, Zhu B, et al. (2007) The burden of depressive symptoms in the long-term treatment of patients with schizophrenia. Schizophr Res 90: 186-197.

Cooper SJ, Reynolds GP, Barnes TRE, et al. (2016) BAP guidelines on the management of weight gain and metabolic disturbances and cardiovascular risk associated with psychosis and antipsychotic drug treatment. J Psychopharmacology 30: 717-748.

Corrigan PW, Rafacz J and Rüsch N (2011) Examining a progressive model of self-stigma and its impact on people with serious mental illness. Psychiatry Res 189: 339-343.

Corcoran C, Malaspina D and Hercher L (2005) Prodromal interventions for schizophrenia vulnerability: The risks of being "at risk". Schizophr Res 73: 173-184.

Corcoran C (2016) Ethical and epidemiological dimensions of labeling psychosis risk. AMA J Ethics 18: 633-642.

Cornblatt BA, Lencz T, Smith CW, et al. (2007) Can antidepressants be used to treat the schizophrenia prodrome? Results of a prospective, naturalistic treatment study of adolescents. J Clin Psychiatry 68: $546-557$.

Correll CU (2010) From receptor pharmacology to improved outcomes: Individualising the selection, dosing, and switching of antipsychotics. Eur Psychiatry 25(Suppl 2): S12-S21.

Correll CU, Rummel-Kluge C, Corves C, et al. (2009) Antipsychotic combinations vs monotherapy in schizophrenia: a meta-analysis of randomized controlled trials. Schizophr Bull 35: 443-457.

Correll CU, Citrome L, Haddad PM, et al. (2016) The use of long-acting injectable antipsychotics in schizophrenia: Evaluating the evidence. J Clin Psychiatry 77(Suppl 3): 1-24.
Correll CU, Robinson DG and Schooler NR (2014) Cardiometabolic risk in patients with first-episode schizophrenia spectrum disorders. JAMA Psychiatry 71: 1350-1363.

Correll CU, Rubio JM and Kane JM (2018) What is the risk-benefit ratio of long-term antipsychotic treatment in people with schizophrenia? World Psychiatry 17: 149-160.

Correll CU, Rubio JM, Inczedy-Farkas G, et al. (2017a) Efficacy of 42 pharmacologic cotreatment strategies added to antipsychotic monotherapy in schizophrenia: Systematic overview and quality appraisal of the meta-analytic evidence. JAMA Psychiatry 74: 675-684.

Correll CU, Solmi M, Veronese N, et al. (2017b) Prevalence, incidence and mortality from cardiovascular disease in patients with pooled and specific severe mental illness: A large-scale meta-analysis of 3,211,768 patients and 113,383,368 controls. World Psychiatry 16: $163-180$.

Couchman L, Bowskill SV, Handley S, et al. (2013) Plasma clozapine and norclozapine in relation to prescribed dose and other factors in patients aged $<18$ years: Data from a therapeutic drug monitoring service, 1994-2010. Early Interv Psychiatry 7: 122-130.

Couchman L, Morgan PE, Spencer EP, et al. (2010) Plasma clozapine, norclozapine, and the clozapine: Norclozapine ratio in relation to prescribed dose and other factors: Data from a therapeutic drug monitoring service, 1993-2007. Ther Drug Monit 32: 438-447.

Crawford MJ, Jayakumar S, Lemmey SJ, et al. (2014) Assessment and treatment of physical health problems among people with schizophrenia: National cross-sectional study. Br J Psychiatry 205: 473-477.

Crespo-Facorro B, Pérez-Iglesias R, Mata I, et al. (2012) Long-term (3-year) effectiveness of haloperidol, risperidone and olanzapine: Results of a randomized, flexible-dose, open-label comparison in first-episode nonaffective psychosis. Psychopharmacology 219: $225-233$.

Crump C, Winkleby MA, Sundquist K, et al. (2013) Comorbidities and mortality in persons with schizophrenia: A Swedish national cohort study. Am J Psychiatry 170: 324-333

Csernansky JG and Schuchart EK (2002) Relapse and rehospitalisation rates in patients with schizophrenia: Effects of second generation antipsychotics. CNS Drugs 16: 473-484.

Cubeddu LX (2016) Drug-induced inhibition and trafficking disruption of ion channels: Pathogenesis of QT abnormalities and drug-induced fatal arrhythmias. Curr Cardiol Rev 12: 141-154.

Cui L, Li M, Deng W, et al. (2011) Overlapping clusters of gray matter deficits in paranoid schizophrenia and psychotic bipolar mania with family history. Neurosci Lett 489: 94-98.

Curson DA, Barnes TRE, Bamber RW, et al. (1985a) Long-term depot maintenance of chronic schizophrenic outpatients: The seven year follow-up of the Medical Research Council fluphenazine/placebo trial 1. Course of illness, stability of diagnosis, and the role of a special maintenance clinic. Br J Psychiatry 146: 464-469.

Curson DA, Barnes TRE, Bamber RWK, et al. (1985b) Long-term depot maintenance of chronic schizophrenic outpatients: The seven year follow-up of the MRC fluphenazine/placebo trial. III. Relapse postponement or relapse prevention? The implications for long-term outcome. Br J Psychiatry 146: 474-480.

Czobor P, Van Dorn RA, Citrome L, et al. (2015) Treatment adherence in schizophrenia: A patient-level meta-analysis of combined CATIE and EUFEST studies. Eur Neuropsychopharmacol 25: 1158-1166.

D'Albis MA and Houenou J (2014) The Kraepelinian dichotomy viewed by neuroimaging. Schizophr Bull 41: 330-335.

Davies C, Cipriani A, Ioannidis J, et al. (2018a) Lack of evidence to favor specific preventive interventions in psychosis: A network meta-analysis. World Psychiatry 17: 196-209.

Davies C, Radua J, Cipriani A, et al. (2018b) Efficacy and acceptability of interventions for attenuated positive psychotic symptoms in individuals at clinical high risk of psychosis: A network meta-analysis. FrontPsychiatry 9: 187. 
Davis JM (1985) Maintenance therapy and the natural course of schizophrenia. J Clin Psychiatry 46: 18-21.

Davis JM and Chen N (2004) Dose response and dose equivalence of antipsychotics. J Clin Psychopharmacol 24: 192-208.

Davis JM, Kane JM, Marder SR, et al. (1993) Dose response of prophylactic antipsychotics. J Clin Psychiatry 54(Suppl): 24-30.

Davis KAS, Farooq S, Hayes J, et al. (2019) Pharmacoepidemiology research: Delivering evidence about drug safety and effectiveness in mental health. Lancet Psychiatry, in press.

Day JC, Bentall RP, Roberts C, et al. (2005) Attitudes toward antipsychotic medication: the impact of clinical variables and relationships with health professionals. Arch Gen Psychiatry 62: 717-724.

Dazzan P, Arango C, Fleischacker W, et al. (2015) Magnetic resonance imaging and the prediction of outcome in first-episode schizophrenia: A review of current evidence and directions for future research. Schizophr Bull 41: 574-583.

De Boer J, Prikken M, Lei WU, et al. (2018) The effect of raloxifene augmentation in men and women with a schizophrenia spectrum disorder: A systematic review and meta-analysis. NPJ Schizophr 4: 1 .

de Deyn PP, Katz IR, Brodtay H, et al. (2005) Management of agitation, aggression, and psychosis associated with dementia: A pooled analysis including three randomized, placebo-controlled double-blind trials in nursing home residents treated with risperidone. Clin Neurol Neurosurg 107: 497-508.

De Hert M, Correll CU and Cohen D. (2010) Do antipsychotic medications reduce or increase mortality in schizophrenia? A critical appraisal of the FIN-11 study. Schizophr Res 117: 68-74.

De Hert M, Detraux J and Peuskens J (2014) Second-generation and newly approved antipsychotics, serum prolactin levels and sexual dysfunctions: A critical literature review. Expert Opin Drug Saf 13: 605-624.

De Hert M, Detraux J and Stubbs B (2016a) Relationship between antipsychotic medication, serum prolactin levels and osteoporosis/osteoporotic fractures in patients with schizophrenia: A critical literature review. Expert Opin Drug Saf 15: 809-823.

De Hert M, Peuskens J, Sabbe T, et al. (2016b) Relationship between prolactin, breast cancer risk, and antipsychotics in patients with schizophrenia: A critical review. Acta Psychiatr Scand 133: 5-22.

De Hert M, Sermon J, Geerts P, et al. (2015) The use of continuous treatment versus placebo or intermittent treatment strategies in stabilized patients with schizophrenia: A systematic review and meta-analysis of randomized controlled trials with first- and second-generation antipsychotics. CNS Drugs 29: 637-658.

de Leon J and Diaz FJ (2005) A meta-analysis of worldwide studies demonstrates an association between schizophrenia and tobacco smoking behaviors. Schizophr Res 76: 135-157.

de Leon J, Diaz FJ, Josiassen RC, et al. (2005) Does clozapine decrease smoking? Prog Neuropsychopharmacol Biol Psychiatry 29: 757762 .

Deakin B, Suckling J, Barnes TRE, et al. (2018) The benefit of minocycline on negative symptoms of schizophrenia in patients with recent-onset psychosis (BeneMin): A randomised, double-blind, placebo-controlled trial. Lancet Psychiatry 5: 885-894.

Demjaha A, Lappin JM, Stahl D, et al. (2017) Antipsychotic treatment resistance in first-episode psychosis: Prevalence, subtypes and predictors. Psychol Med 47: 1981-1989.

Demjaha A, Morgan K, Morgan C, et al. (2009) Combining dimensional and categorical representation of psychosis: The way forward for DSM-V and ICD-11? Psychol Med 39: 1943-1955.

Demjaha A, Murray RM, McGuire PK, Kapur S, et al. (2012) Dopamine synthesis capacity in patients with treatment-resistant schizophrenia. Am J Psychiatry 169: 1203-1210.

Denison FC, Aedla NR, Keag O, Hor K, et al. (2019) Care of women with obesity in pregnancy. Green-top Guideline No. 72. BJOG 126: e62-e106.
Dettling M, Sachse C, Brockmöller J, et al. (2000) Long-term therapeutic drug monitoring of clozapine and metabolites in psychiatric in- and outpatients. Psychopharmacology (Berl)152: 80-86.

Diaz E, Neuse E, Sullivan MC, et al. (2004) Adherence to conventional and atypical antipsychotics after hospital discharge. J Clin Psychiatry 65: 354-360.

Dickinson D, Ramsey ME and Gold JM (2007) Overlooking the obvious: A meta-analytic comparison of digit symbol coding tasks and other cognitive measures in schizophrenia. Arch Gen Psychiatry 64: $532-542$.

DiMatteo MR (2004) Evidence-based strategies to foster adherence and improve patient outcomes. JAAPA 17: 18-21.

Dold M, Fugger G, Aigner M, et al. (2015) Dose escalation of antipsychotic drugs in schizophrenia: A meta-analysis of randomized controlled trials. Schizophr Res 166: 187-193.

Dold M and Leucht S (2014) Pharmacotherapy of treatment-resistant schizophrenia: A clinical perspective. Evid Based Ment Health 17: 33-37.

Dolder CR, Lacro JP, Dunn LB, et al. (2002) Antipsychotic medication adherence: is there a difference between typical and atypical agents? Am J Psychiatry 159: 103-108.

Dolder CR, Lacro JP, Warren KA, et al. (2004) Brief evaluation of medication influences and beliefs: Development and testing of a brief scale for medication adherence. J Clin Psychopharmacol 24: 404-409.

Dollfus S, Mach C and Morello R (2016) Self-evaluation of negative symptoms: A novel tool to assess negative symptoms. Schizophr Bull 42: 571-578.

Drake RJ, Haley CJ, Akhtar S, et al. (2000) Causes and consequences of duration of untreated psychosis in schizophrenia. Br J Psychiatry 177: 511-515.

Drake RJ, Nordentoft M, Haddock G, et al. (2015) Modeling determinants of medication attitudes and poor adherence in early nonaffective psychosis: Implications for intervention. Schizophr Bull 41: 584-596.

Druss BG, Von Esenwein SA, Glick GE, et al. (2017) Randomized trial of an integrated behavioral health home: The health outcomes management and evaluation (HOME) study. Am J Psychiatr 174: 246-255.

D'Souza DC, Radhakrishnan R, Perry E, et al. (2013) Feasibility, safety, and efficacy of the combination of D-serine and computerized cognitive retraining in schizophrenia: An international collaborative pilot study. Neuropsychopharmacology 38: 492-503.

Durst R, Teitelbaum A, Katz G, et al. (1999) Withdrawal from clozapine: The "rebound phenomenon". Isr J Psychiatry Relat Sci 36: 122-128.

Dutta R, Murray RM, Allardyce J, et al. (2011) Early risk factors for suicide in an epidemiological first episode psychosis cohort. Schizophr Res 126: 11-19.

Dutta R, Murray RM, Allardyce J, et al. (2012) Mortality in first-contact psychosis patients in the U.K.: A cohort study. Psychol Med 42: 1649-1661.

Dutta R, Murray RM, Hotopf M, et al. (2010) Reassessing the long-term risk of suicide after a first episode of psychosis. Arch Gen Psychiatry 67: 1230-1237.

Earls HA, Curran T and Mittal V (2016) A meta-analytic review of auditory event-related potential components as endophenotypes for schizophrenia: Perspectives from first-degree relatives. Schizophr Bull 42: 1504-1516.

Eisner E, Drake R, Lobban F, et al. (2018) Comparing early signs and basic symptoms as methods for predicting psychotic relapse in clinical practice. Schizophr Res 192: 124-130.

Eisner E, Drake R and Barrowclough C (2013) Assessing early signs of relapse in psychosis: Review and future directions. Clin Psychol Rev 33: 637-653.

Ekblom B, Eriksson K and Lindström LH (1984) Supersensitivity psychosis in schizophrenic patients after sudden clozapine withdrawal. Psychopharmacology (Berl) 83: 293-294.

El-Sayeh HG, Morganti C and Adams CE (2006) Aripiprazole for schizophrenia. Systematic review. Br J Psychiatry 189: 102-108. 
Emsley R, Chiliza B, Asmal L, et al. (2013a) The nature of relapse in schizophrenia. BMC Psychiatry 13: 50.

Emsley R, Nuamah I, Gopal S, et al. (2018) Relapse after antipsychotic discontinuation in schizophrenia as a withdrawal phenomenon vs illness recurrence: A post hoc analysis of a randomized placebo-controlled study. J Clin Psychiatry 79: pii: $17 \mathrm{~m} 11874$.

Emsley R, Oosthuizen P, Koen L, et al. (2008) Remission in patients with first-episode schizophrenia receiving assured antipsychotic medication: A study with risperidone long-acting injection. Int Clin Psychopharmacol 23: 325-331.

Emsley R, Oosthuizen P, Koen L, et al. (2013b) Comparison of treatment response in second-episode versus first-episode schizophrenia. J Clin Psychopharmacol 33: 80-83.

Emsley RA, Buckley P, Jones AM, et al. (2003) Differential effect of quetiapine on depressive symptoms in patients with partially responsive schizophrenia. J Psychopharmacol 17: 210-215.

Emul M and Kalelioglu T (2015) Etiology of cardiovascular disease in patients with schizophrenia: Current perspectives. Neuropsychiatr Dis Treat 11: 2493-503.

Ennis ZN and Damkier P (2015) Pregnancy exposure to olanzapine, quetiapine, risperidone, aripiprazole and risk of congenital malformations. A systematic review. Basic Clin Pharmacol Toxicol 116: 315-320.

Erro R, Bhatia KP and Tinazzi M (2015) Parkinsonism following neuroleptic exposure: A double-hit hypothesis? Mov Disord 30: 780-785.

Essali A, Al-Haj Haasan N, Li C, et al. (2009) Clozapine versus typical neuroleptic medication for schizophrenia. Cochrane Database Syst Rev 2: CD000059.

Essock SM, Covell NH, Davis SM, et al. (2006) Effectiveness of switching antipsychotic medications. Am J Psychiatry 163: 2090-2095.

European Medicines Agency (2018) New Measures to Avoid Valproate Exposure in Pregnancy Endorsed. Available at: https://www.ema. europa.eu/en/documents/referral/valproate-article-31-referral-newmeasures-avoid-valproate-exposure-pregnancy-endorsed_en-0.pdf

Evans-Lacko S, Brohan E, Mojtabai R, et al. (2012) Association between public views of mental illness and self-stigma among individuals with mental illness in 14 European countries. Psychol Med 42: 1741-1752.

Faber G, Smid HG, Van Gool AR, et al. (2012) The effects of guided discontinuation of antipsychotics on neurocognition in first onset psychosis. Eur Psychiatry 27: 275-280.

Falloon IR, Boyd JL, McGill CW, et al. (1985) Family management in the prevention of morbidity of schizophrenia. Clinical outcome of a two-year longitudinal study. Arch Gen Psychiatry 42: 887-896.

Falkenberg I, Benetti S, Raffin M, et al. (2017) Clinical utility of magnetic resonance imaging in first-episode psychosis. Br J Psychiatry 211: 231-237.

Faries DE, Ascher-Svanum H, Nyhuis AW, et al. (2009) Clinical and economic ramifications of switching antipsychotics in the treatment of schizophrenia. BMC Psychiatry 9: 54-62.

Farokhnia M, Azarkolah A, Adinehfar F, et al. (2013) N-acetylcysteine as an adjunct to risperidone for treatment of negative symptoms in patients with chronic schizophrenia: A randomized, double-blind, placebo-controlled study. Clin Neuropharmacol 36: 185-192.

Faulkner A, Maguire P and Regnard C (1994) Breaking bad news - a flow diagram. Palliat Med 8: 145-151.

Fayek M, Kingsbury SJ, Zada J, et al. (2001) Cardiac effects of antipsychotic medications. Psychiatr Serv 52: 607-609.

Fazel S, Gulati G, Linsell L, et al. (2009) Schizophrenia and violence: Systematic review and meta-analysis. PLoS Med 6: e1000120.

Fazel S, Zetterqvist J, Larsson H, et al. (2014) Antipsychotics, mood stabilisers, and risk of violent crime. Lancet 384: 1206-1214.

Feigenson KA, Kusnecov AW and Silverstein SM (2014) Inflammation and the two-hit hypothesis of schizophrenia. Neurosci Biobehav Rev 38: 72-93.
Fenton WS, Blyler CR and Heinssen RK (1997) Determinants of medication compliance in schizophrenia: Empirical and clinical findings. Schizophr Bull 23: 637-651.

Fernandez HH, Factor SA, Hauser RA, et al. (2017) Randomised controlled trial of deutetrabenazine for tardive dyskinesia: The ARMTD study. Neurology 88: 2003-2010.

Fialko L, Garety PA, Kuipers E, et al. (2008) A large-scale validation study of the Medication Adherence Rating Scale (MARS). Schizophr Res 100: 53-59.

Flanagan RJ, Yusufi B and Barnes TR (2003) Comparability of wholeblood and plasma clozapine and norclozapine concentrations. $\mathrm{Br} J$ Clin Pharmacol 56: 135-138.

Fleischhacker WW, Heikkinen ME, Olié JP, et al. (2010) Effects of adjunctive treatment with aripiprazole on body weight and clinical efficacy in schizophrenia patients treated with clozapine: A randomized, double-blind, placebo-controlled trial. Int J Neuropsychopharmacol 13: 1115-1125.

Fleischhacker WW and Uchida H (2014) Critical review of antipsychotic polypharmacy in the treatment of schizophrenia. Int J Neuropsychopharmacol 17: 1083-1093.

Foley DL and Morley KI (2011) Systematic review of early cardiometabolic outcomes of the first treated episode of psychosis. Arch Gen Psychiatry 68: 609-616.

Foubert-Samier A, Helmer C, Perez F, et al. (2012) Past exposure to neuroleptic drugs and risk of Parkinson disease in an elderly cohort. Neurology 79: 1615-1621.

Freedman R (2014) Alpha7-nicotinic acetylcholine receptor agonists for cognitive enhancement in schizophrenia. Annu Rev Med 65: 245-261.

Frei K, Truong DD, Fahn S, et al. (2018) The nosology of tardive syndromes. J Neurol Sci 389: 10-16.

Freudenreich O, Henderson DC, Walsh JP, et al. (2007) Risperidone augmentation for schizophrenia partially responsive to clozapine: A double-blind, placebo-controlled trial. Schizophr Res 92: 90-94.

Friedman RA (2006) Violence and mental illness - how strong is the link? N Engl J Med 355: 2064-2066.

Froes Brandao D, Strasser-Weippl K and Goss PE (2016) Prolactin and breast cancer: The need to avoid undertreatment of serious psychiatric illnesses in breast cancer patients: A review. Cancer 122: 184-188.

Frogley C, Taylor D, Dickens G, et al. (2012) A systematic review of the evidence of clozapine's anti-aggressive effects. Int J Neuropsychopharmacol 15: 1351-1371.

Fu D-J, Turkoz I, Simonson RB, et al. (2015) Paliperidone palmitate oncemonthly reduces risk of relapse of psychotic, depressive, and manic symptoms and maintains functioning in a double-blind, randomized study of schizoaffective disorder. J Clin Psychiatry 76: 253-262.

Funk KA and Bostwick JR (2013) A comparison of the risk of QT prolongation among SSRIs. Ann Pharmacother 47: 1330-1341.

Fusar-Poli P, Bonoldi I, Yung AR, et al. (2012) Predicting psychosis: Meta-analysis of transition outcomes in individuals at high clinical risk. Arch Gen Psychiatry 69: 220-229.

Fusar-Poli P, Borgwardt S, Bechdolf A, et al. (2013a) The psychosis high-risk state: A comprehensive state-of-the-art review. JAMA Psychiatry 70: 107-120.

Fusar-Poli P, Byrne M, Badger S, et al. (2013b) Outreach and support in South London (OASIS), 2001-2011: Ten years of early diagnosis and treatment for young individuals at high clinical risk for psychosis. Eur Psychiatry 28: 315-326.

Fusar-Poli P, Nelson B, Valmaggia L, et al. (2014) Comorbid depressive and anxiety disorders in 509 individuals with an at-risk mental state: Impact on psychopathology and transition to psychosis. Schizophr Bull 40: 120-131.

Fusar-Poli P, Papanastasiou E, Stahl D, et al. (2015) Treatments of negative symptoms in schizophrenia: Meta-analysis of 168 randomized placebo-controlled trials. Schizophr Bull 41: 892-899. 
Fusar-Poli P, Valmaggia L and McGuire P (2007) Can antidepressants prevent psychosis? Lancet 370: 1746-1748.

Gaebel W, Frick U, Köpcke W, et al. (1993) Early neuroleptic intervention in schizophrenia: Are prodromal symptoms valid predictors of relapse? Br J Psychiatry Suppl 21: 8-12.

Gaebel W, Riesbeck M, Wölwer W, et al. (2011) Relapse prevention in first-episode schizophrenia - maintenance vs intermittent drug treatment with prodrome-based early intervention: Results of a randomized controlled trial within the German Research Network on Schizophrenia. J Clin Psychiatry 72: 205-218.

Gaebel W, Riesbeck M, Wölwer W, et al. (2014) Rates and predictors of remission in first-episode schizophrenia within 1 year of antipsychotic maintenance treatment. Results of a randomized controlled trial within the German Research Network on Schizophrenia. Schizophr Res 152: 478-486.

Gaebel W, Weinmann S, Sartorius N, et al. (2005) Schizophrenia practice guidelines: International survey and comparison. Br J Psvchiatry 187: 248-255.

Galbally M, Frayne J, Watson SJ, et al. (2019) Psychopharmacological prescribing practices in pregnancy for women with severe mental illness: A multicentre study. Eur Neuropsychopharmacol 29: 57-65.

Galderisi S, Mucci A, Buchanan RW, et al. (2018) Negative symptoms of schizophrenia: New developments and unanswered research questions. Lancet Psychiatry 5: 664-677.

Gallego JA, Bonetti J, Zhang J, et al. (2012) Prevalence and correlates of antipsychotic polypharmacy: A systematic review and meta-regression of global and regional trends from the 1970s to 2009. Schizophr Res 138: 18-28.

Galling B, Roldan A, Hagi K, et al. (2017) Antipsychotic augmentation vs. monotherapy in schizophrenia: Systematic review, meta-analysis and meta-regression analysis. World Psychiatry 16: 77-89.

Galling B, Vernon JA, Pagsberg AK, et al. (2018) Efficacy and safety of antidepressant augmentation of continued antipsychotic treatment in patients with schizophrenia. Acta Psychiat Scand 137: 187-205.

Ganguli R, Brar JS, Mahmoud R, et al. (2008) Assessment of strategies for switching patients from olanzapine to risperidone: A randomized, open-label, rater-blinded study. BMC Med 6: 17-10.

Gao SY, Wu QJ, Sun C, et al. (2018) Selective serotonin reuptake inhibitor use during early pregnancy and congenital malformations: A systematic review and meta-analysis of cohort studies of more than 9 million births. BMC Med 16: 205.

García-Cabeza I, Gómez JC, Sacristán JA, et al. (2001) Subjective response to antipsychotic treatment and compliance in schizophrenia. A naturalistic study comparing olanzapine, risperidone and haloperidol (EFESO Study). BMC Psychiatry 1: 7.

Gardner DM, Murphy AL, O'Donnell H, et al. (2010) International consensus study of antipsychotic dosing. Am J Psychiatry 167: 686-693.

Gardner-Sood P, Lally J, Smith S, et al. (2015) Cardiovascular risk factors and metabolic syndrome in people with established psychotic illnesses: Baseline data from the IMPaCT randomized controlled trial. Psychol Med 45: 2619-2629.

Garety PA, Kuipers E, Fowler D, et al. (2001) A cognitive model of the positive symptoms of psychosis. Psychol Med 31: 189-195.

Gee KW, Olincy A, Kanner R, et al. (2017) First in human trial of a type I positive allosteric modulator of alpha7-nicotinic acetylcholine receptors: Pharmacokinetics, safety, and evidence for neurocognitive effect of AVL-3288. J Psychopharmacol 31: 434-441.

Gee S, Vergunst F, Howes O, et al. (2014) Practitioner attitudes to clozapine initiation. Acta Psychiatr Scand 130: 16-24.

Genç Y, Taner E and Candansayar S (2007) Comparison of clozapineamisulpride and clozapine-quetiapine combinations for patients with schizophrenia who are partially responsive to clozapine: A singleblind randomized study. Adv Ther 24: 1-13.

Ghaemi SN and Thommi SB (2010) Death by confounding: Bias and mortality. Int J Clin Pract 64: 1009-1014.
Gilbert PL, Harris MJ, McAdams LA, et al. (1995) Neuroleptic withdrawal in schizophrenic patients. A review of the literature. Arch Gen Psychiatry 52: 173-188.

Gillespie AL, Samanaite R, Mill J, et al. (2017) Is treatment-resistant schizophrenia categorically distinct from treatment-responsive schizophrenia? A systematic review. BMC Psychiatry 17: 12.

Girardin FR, Gex-Fabry M, Berney P, et al. (2013) Drug-induced long QT in adult psychiatric inpatients: The 5-year cross-sectional ECG screening outcome in psychiatry study. Am J Psychiatry 170: 1468-1476.

Girgis RR, Phillips MR, Li X, et al. (2011) Clozapine v. chlorpromazine in treatment-naive, first-episode schizophrenia: 9-year outcomes of a randomised clinical trial. Br J Psychiatry 199: 281-288.

Gitlin M, Nuechterlein K, Subotnik KL, et al. (2001) Clinical outcome following neuroleptic discontinuation in patients with remitted recent-onset schizophrenia. Am J Psychiatry 158: 1835-1842.

Glazer WM, Morgenstern H and Doucette JT (1993) Predicting the longterm risk of tardive dyskinesia in outpatients maintained on neuroleptic medications. J Clin Psychiatry 54: 133-139.

Gleeson JF, Rawlings D, Jackson HJ, et al. (2005) Early warning signs of relapse following a first episode of psychosis. Schizophr Res 80: $107-111$.

Glick ID, Zaninelli R, Hsu C, et al. (2004) Patterns of concomitant psychotropic medication use during a 2-year study comparing clozapine and olanzapine for the prevention of suicidal behavior. J Clin Psychiatry 65: 679-685.

Godleski LS and Sernyak MJ (1996) Agranulocytosis after addition of risperidone to clozapine treatment. Am J Psychiatry 153: 735-736.

Goff DC (2017) D-cycloserine in schizophrenia: New strategies for improving clinical outcomes by enhancing plasticity. Curr Neuropharmacol 15: 21-34.

Goff DC, Cather C, Gottlieb JD, et al. (2008) Once-weekly D-cycloserine effects on negative symptoms and cognition in schizophrenia: An exploratory study. Schizophr Res 106: 320-327.

Goff DC, Falkai P, Fleischhacker WW, et al. (2017) The long-term effects of antipsychotic medication on clinical course in schizophrenia. Am J Psychiatry 174: 840-849.

Goldberg TE, Goldman RS, Burdick KE, et al. (2007) Cognitive improvement after treatment with second-generation antipsychotic medications in first-episode schizophrenia: Is it a practice effect? Arch Gen Psychiatry 64: 1115-1122.

Goldsmith DR, Rapaport MH and Miller BJ (2016) A meta-analysis of blood cytokine network alterations in psychiatric patients: Comparisons between schizophrenia, bipolar disorder and depression. Mol Psychiatry 21: 1696-1709.

Goodwin G, Haddad P, Ferrier I, et al. (2016) Evidence-based guidelines for treating bipolar disorder: Revised third edition recommendations from the British Association for Psychopharmacology. Psychopharmacol 30: 495-553.

Gotfredsen DR, Wils RS, Hjorthøj C, et al. (2017) Stability and development of psychotic symptoms and the use of antipsychotic medication - long-term follow-up. Psychol Med 47: 2118-2129.

Goudie AJ (2000) What is the clinical significance of the discontinuation syndrome seen with clozapine? J Psychopharmacol 14: 188-192.

Gray R, Bressington D, Ivanecka A, et al. (2016) Is adherence therapy an effective adjunct treatment for patients with schizophrenia spectrum disorders? A systematic review and meta-analysis. BMC Psychiatry 16: 90 .

Gray R, Leese M, Bindman J, et al. (2006) Adherence therapy for people with schizophrenia. Br J Psychiatry 189: 508-514.

Gray SL, Anderson ML, Dublin S, et al. (2015) Cumulative use of strong anticholinergics and incident dementia: A prospective cohort study. JAMA Intern Med 175: 401-407.

Greenberg WM and Citrome L (2015) Paliperidone palmitate for schizoaffective disorder: A review of the clinical evidence. Neurol Ther 4: 81-91. 
Greenberg WM and Citrome L (2017) Pharmacokinetics and pharmacodynamics of lurasidone hydrochloride, a second-generation antipsychotic: A systematic review of the published literature. Clin Pharmacokinet 56: 493-503.

Gregory A, Mallikarjun P and Upthegrove R (2017) Treatment of depression in schizophrenia: Systematic review and meta-analysis. $\mathrm{Br} J$ Psychiatry 211: 198-204.

Grinshpoon A, Moskowitz M, Valevski A, et al. (1998) Zuclopenthixol, $\mathrm{D}(1) / \mathrm{D}(2)$ antagonist, for treatment of chronic aggressive schizophrenia and psychotic oligophrenic patients. Eur Psychiatry 13: 273-275.

Gruber D, Südmeyer M, Deuschl G, et al. (2018) Neurostimulation in tardive dystonia/dyskinesia: A delayed start, sham stimulation-controlled randomized trial. Brain Stimul 11: 1368-1377.

Gurrera RJ (1999) Sympathoadrenal hyperactivity and the etiology of neuroleptic malignant syndrome. Am J Psychiatry 156: 169-180.

Gurrera RJ (2017) A systematic review of sex and age factors in neuroleptic malignant syndrome diagnosis frequency. Acta Psychiatr Scand 135: 398-408.

Gurrera RJ, Caroff SN, Cohen A, et al. (2011) An international consensus study of neuroleptic malignant syndrome diagnostic criteria using the Delphi method. J Clin Psychiatry 72: 1222-1228.

Gurvich C, Hudaib A, Gavrilidis E, et al. (2019) Raloxifene as a treatment for cognition in women with schizophrenia: The influence of menopause status. Psychoneuroendocrinology 100: 113-119.

Guy W (1976) Clinical Global Impressions. ECDEU Assessment Manual for Psychopharmacology - Revised. U.S. Department of Health, Education, and Welfare; Public Health Service, Alcohol; Drug Abuse, and Mental Health Administration; National Institute of Mental Health; Psychopharmacology Research Branch; Division of Extramural Research Programs, Rockville, MD, US, 218-222.

Habermann F, Fritzsche J, Fuhlbruck F, et al. (2013) Atypical antipsychotic drugs and pregnancy outcome: A prospective cohort study. $J$ Clin Psychopharmacol 33: 453-462.

Hackinger S, Prins B, Mamakou V, et al. (2018) Evidence for genetic contribution to the increased risk of type 2 diabetes in schizophrenia. Transl Psychiatry 8: 252.

Haddad PM, Brain C and Scott J (2014) Nonadherence with antipsychotic medication in schizophrenia: Challenges and management strategies. Patient Relat Outcome Meas 5: 43-62.

Haddad PM and Correll CU (2018) The acute efficacy of antipsychotics in schizophrenia: A review of recent meta-analyses. Ther Adv Psychopharmacol 8: 303-318.

Haddad PM and Sharma SG (2007) Adverse effects of atypical antipsychotics: Differential risk and clinical implications. CNS Drugs 21: 911-936.

Haddad PM, Taylor M and Niaz OS (2009) First-generation antipsychotic long-acting injections $\mathrm{v}$. oral antipsychotics in schizophrenia: Systematic review of randomised controlled trials and observational studies. Br J Psychiatry 195: s20-s28.

Haddad PM and Wieck A (2004) Antipsychotic-induced hyperprolactinaemia: Mechanisms, clinical features and management. Drugs 64: $2291-2314$

Häfner H (2005) Schizophrenia and depression. Eur Arch Psychiatry Clin Neurosci 255: 157-158.

Häfner H, Maurer K, Trendler G, et al. (2005) Schizophrenia and depression: Challenging the paradigm of two separate diseases-a controlled study of schizophrenia, depression and healthy controls. Schizophr Res 77: 11-24.

Haig GM, Bain EE, Robieson WZ, et al. (2016) A randomized trial to assess the efficacy and safety of ABT-126, a selective alpha7 nicotinic acetylcholine receptor agonist, in the treatment of cognitive impairment in schizophrenia. Am J Psychiatry 173: 827-835.

Hale T (2012) Medications and Mother's Milk. Amarillo: Hale Publishing.
Haloperidol decanoate SmPC. Available at: https://www.medicines.org. uk/emc/product/968/smpc (accessed September 2019)

Hamann J, Cohen R, Leucht S, et al. (2007) Shared decision making and long-term outcome in schizophrenia treatment. J Clin Psychiatry 68: 992-997.

Hanley GE, Smolina K, Mintzes B, et al. (2016) Postpartum haemorrhage and use of serotonin reuptake inhibitors in pregnancy. Obstet Gynecol 127: 553-561.

Hanssens L, De Hert M, Kalnicka D, et al. (2007) Pharmacological treatment of severe dyslipidaemia in patients with schizophrenia. Int Clin Psychopharmacol 22: 43-49.

Harrigan SM, McGorry PD and Krstev H (2003) Does treatment delay in first-episode psychosis really matter? Psychol Med 33: 97-110.

Harrow M, Jobe TH and Faull RN (2014) Does treatment of schizophrenia with antipsychotic medications eliminate or reduce psychosis? A 20-year multi-follow-up study. Psychol Med 44: 3007-3016.

Hartmann JA, Yuen HP, McGorry PD, et al. (2016) Declining transition rates to psychotic disorder in "ultra-high risk" clients: Investigation of a dilution effect. Schizophr Res 170: 130-136.

Harvey PD, Koren D, Reichenberg A, et al. (2006) Negative symptoms and cognitive deficits: What is the nature of their relationship? Schizophr Bull 32: 250-258.

Harvey PD, Siu CO, Hsu J, et al. (2013) Effect of lurasidone on neurocognitive performance in patients with schizophrenia: A short-term placebo- and active-controlled study followed by a 6-month doubleblind extension. Eur Neuropsychopharmacol 23: 1373-1382.

Hasan A, Falkai P, Wobrock T, et al. (2012) World Federation of Societies of Biological Psychiatry (WFSBP) guidelines for biological treatment of schizophrenia, part 1: Update 2012 on the acute treatment of schizophrenia and the management of treatment resistance. World $J$. Biol Psychiatry 13: 318-378.

Hashimoto N, Toyomaki A, Honda M, et al. (2015) Long-term efficacy and tolerability of quetiapine in patients with schizophrenia who switched from other antipsychotics because of inadequate therapeutic response-a prospective open-label study. Ann Gen Psychiatr 14: 1 .

Hatta K, Sugiyama N and Ito H (2018) Switching and augmentation strategies for antipsychotic medications in acute-phase schizophrenia: Latest evidence and place in therapy. Ther Adv Psychopharmacol 8: $173-183$

Hauser RA, Factor SA, Marder SR, et al. (2017) KINECT 3: A phase 3 randomised, double-blind, placebo-controlled trial of valbenazine for tardive dyskinesia. Am J Psychiatry 174: 476-484.

Hay J (1995) Complications at site of injection of depot neuroleptics. BMJ 311: 421.

Hayes JF, Marston L, Walters K, et al. (2017) Mortality gap for people with bipolar disorder and schizophrenia: UK-based cohort study 2000-2014. Br J Psychiatry 211: 175-181.

Hayhurst KP, Drake RJ, Massie JA, et al. (2014) Improved quality of life over one year is associated with improved adherence in patients with schizophrenia. Eur Psychiatry 29: 191-196.

Hazell L, Raschi E, De Ponti F, et al. (2017) Evidence for the hERG liability of antihistamines, antipsychotics, and anti-infective agents: A systematic literature review from the ARITMO Project. $J$ Clin Pharmacol 57: 558-572.

Hecht EM and Landy DC (2012) Alpha-2 receptor antagonist addon therapy in the treatment of schizophrenia; A meta-analysis. Schizophr Res 134: 202-206.

Helfer B, Samara MT, Huhn M, et al. (2016) Efficacy and safety of antidepressants added to antipsychotics for schizophrenia: A systematic review and meta-analysis. Am J Psychiatry 173: 876-886.

Heller HM, Ravelli ACJ, Bruning AHL, et al. (2017) Increased postpartum haemorrhage, the possible relation with serotonergic and other psychopharmacological drugs: A matched cohort study. BMC Pregnancy Childbirth 17: 166. 
Henderson DC and Doraiswamy PM (2008) Prolactin-related and metabolic adverse effects of atypical antipsychotic agents. J Clin Psychiatry 69(Suppl 1): 32-44.

Henderson DC, Fan X, Copeland PM, et al. (2009) Ziprasidone as an adjuvant for clozapine- or olanzapine-associated medical morbidity in chronic schizophrenia. Hum Psychopharmacol 24: 225-32.

Hennekens CH, Hennekens AR, Hollar D, et al. (2005) Schizophrenia and increased risks of cardiovascular disease. Am Heart $J 150$ : $1115-1121$

Heres S, Hamann J, Kissling W, et al. (2006) Attitudes of psychiatrists toward antipsychotic depot medication. J Clin Psychiatry 67: 19481953.

Heringa SM, Begemann MJ, Goverde AJ, et al. (2015) Sex hormones and oxytocin augmentation strategies in schizophrenia: A quantitative review. Schizophr Res 168: 603-613.

Herings RM and Erkens JA (2003) Increased suicide attempt rate among patients interrupting use of atypical antipsychotics. Pharmacoepidemiol Drug Saf 12: 423-424.

Herlihy D, Chatterjee A, Gwenzi S, et al. (2010) Low violence rates on picu despite reduction in use of combination or highdose antipsychotic prescribing. J Psychopharmacol 24(Suppl 3): A14.

Herniman SE, Allott K, Phillips LJ, et al. (2019) Depressive psychopathology in first-episode schizophrenia spectrum disorders: A systematic review, meta-analysis and meta-regression. Psychol Med 49: 2463-2474.

Herold R, Szekeres G and Bitter I (2017) Continuous maintenance antipsychotic treatment in schizophrenia. Psychiatr Hung 32: 296-306.

Herz MI, Glazer WM, Mostert MA, et al. (1991) Intermittent vs maintenance medication in schizophrenia. Two-year results. Arch Gen Psychiatry 48: 333-339.

Hippisley-Cox J, Coupland C, Vinogradova Y, et al. (2008) Predicting cardiovascular risk in England and Wales: Prospective derivation and validation of QRISK2. BMJ 336: 1475-1482.

Hjorthøj C, Stürup AE, McGrath JJ, et al. (2017) Years of potential life lost and life expectancy in schizophrenia: A systematic review and meta-analysis. Lancet Psychiatry 4: 295-301.

Hoang U, Stewart R and Goldacre MJ (2011) Mortality after hospital discharge for people with schizophrenia or bipolar disorder: Retrospective study of linked English hospital episode statistics, 1999-2006. BMJ 343: d5422.

Hogan TP, Awad AG and Eastwood R (1983) A self-report scale predictive of drug compliance in schizophrenics: Reliability and discriminative validity. Psychol Med 13: 177-183.

Hogarty GE, McEvoy JP, Munetz M, et al. (1988) Dose of fluphenazine, familial expressed emotion and outcome in schizophrenia. Arch Gen Psychiatry 45: 797-805.

Holt RI and Mitchell AJ (2015) Diabetes mellitus and severe mental illness: Mechanisms and clinical implications. Nat Rev Endocrinol 11: 79-89.

Holt RIG, Gossage-Worrall R, Hind D, et al. (2019) Structured lifestyle education for people with schizophrenia, schizoaffective disorder and first-episode psychosis (STEPWISE): Randomised controlled trial. Br J Psychiatry 214: 63-73.

Honer WG, Thornton AE, Chen EY, et al. (2006) Clozapine alone versus clozapine and risperidone with refractory schizophrenia. $N$ Engl $J$ Med 354: 472-482.

Hong LE, Thaker GK, McMahon RP, et al. (2011) Effects of moderate-dose treatment with varenicline on neurobiological and cognitive biomarkers in smokers and nonsmokers with schizophrenia or schizoaffective disorder. Arch Gen Psychiatry 68: 1195-1206.

Horvitz-Lennon M, Mattke S, Predmore Z, et al. (2017) The role of antipsychotic plasma levels in the treatment of schizophrenia. Am J Psychiatry 174: 421-426.
Hotham JE, Simpson PJ, Brooman-White RS, et al. (2014) Augmentation of clozapine with amisulpride: An effective therapeutic strategy for violent treatment-resistant schizophrenia patients in a UK highsecurity hospital. CNS Spectr 19: 403-410.

Hou CL, Ma XR, Zang Y, et al. (2016) Antipsychotic polypharmacy and quality of life in patients with schizophrenia treated in primary care in China. Int J Clin Pharmacol Ther 54: 36-42.

Houston JP, Fijal B, Heinloth AN, et al. (2010) Genetic associations of prolactin increase in olanzapine/fluoxetine combination-treated patients. Psychiatry Res 175: 171-172.

Howell S, Yarovova E, Khwanda A, et al. (2019) Cardiovascular effects of psychotic illnesses and antipsychotic therapy. Heart. Epub ahead of print 4 November 2019. doi: 10.1136/ heartjnl-2017-312107.

Howes OD, Egerton A, Allan V, et al. (2009) Mechanisms underlying psychosis and antipsychotic treatment response in schizophrenia: Insights from PET and SPECT imaging. Curr Pharm Des 15: 2550 2559.

Howes OD, Kambeitz J, Kim E, et al. (2012b) The nature of dopamine dysfunction in schizophrenia and what this means for treatment. Arch Gen Psychiatry 69: 776-786.

Howes OD, McCutcheon R, Agid O, et al. (2017) Treatment-resistant schizophrenia: Treatment Response and Resistance in Psychosis (TRRIP) working group consensus guidelines on diagnosis and terminology. Am J Psychiatry 174: 216-229.

Howes OD, Vergunst F, Gee S, et al. (2012a) Adherence to treatment guidelines in clinical practice: Study of antipsychotic treatment prior to clozapine initiation. Br J Psychiatry 201: 481-485.

Huerta-Ramos E, Iniesta R, et al. (2014) Effects of raloxifene on cognition in postmenopausal women with schizophrenia: A double-blind, randomized, placebo-controlled trial. Eur Neuropsychopharmacol 24: 223-231.

Huhn M, Nikolakopoulou A, Schneider-Thoma J, et al. (2019) Comparative efficacy and tolerability of 32 oral antipsychotics for the acute treatment of adults with multi-episode schizophrenia: A systematic review and network meta-analysis. Lancet 394: 939-951.

Hui CLM, Honer WG, Lee EHM, et al. (2018) Long-term effects of discontinuation from antipsychotic maintenance following firstepisode schizophrenia and related disorders: A 10 year followup of a randomised, double-blind trial. Lancet Psychiatry 5: $432-442$.

Hunt GE, Bergen J and Bashir M (2002) Medication compliance and comorbid substance abuse in schizophrenia: Impact on community survival 4 years after a relapse. Schizophr Res 54: 253-264.

Huybrechts KF, Bateman BT, Palmsten K, et al. (2015) Antidepressant use late in pregnancy and risk of persistent pulmonary hypertension of the newborn. JAMA 313: 2142-2151.

Huybrechts KF, Hernandez-Diaz S and Avorn J (2014) Antidepressant use in pregnancy and the risk of cardiac defects. New Engl J Med 371: $1168-1169$

Huybrechts KF, Hernandez-Diaz S, Parorno E, et al. (2016) Antipsychotic use in pregnancy and the risk of congenital malformations. JAMA Psychiatry 73: 938-946.

Hynes C, Keating D, McWilliams S, et al. (2015) Glasgow Antipsychotic Side-effects Scale for Clozapine - Development and validation of a clozapine-specific side-effects scale. Schizophr Res 168(1-2): 505-513.

Iqbal MM, Rahman A, Husain Z, et al. (2003) Clozapine: A clinical review of adverse effects and management. Ann Clin Psychiatry 15: 33-48.

Iqbal Z, Birchwood M, Chadwick P, et al. (2000) Cognitive approach to depression and suicidal thinking in psychosis. 2 . Testing the validity of a social ranking model. Br J Psychiatry 177: 522-528.

Jann MW and Penzak SR (2018) Long-acting injectable second-generation antipsychotics: An update and comparison between agents. CNS Drugs 32: 241-257. 
Jauhar S, Veronese M, Nour MM, et al. (2019) Determinants of treatment response in first-episode psychosis: An 18F-DOPA PET study. Mol Psychiatry 24: 1502-1512.

Jeste DV, Caligiuri MP, Paulson JS, et al. (1995) Risk of tardive dyskinesia in older patients: A prospective longitudinal study of 266 patients. Arch Gen Psychiatry 52: 756-765.

Johannesen L, Vicente J, Mason JW, et al. (2014) Differentiating druginduced multichannel block on the electrocardiogram: Randomized study of dofetilide, quinidine, ranolazine, and verapamil. Clin Pharmacol Ther 96: 549-558.

Johnson DA and Freeman H (1973) Drug defaulting by patients on longacting phenothiazines. Psychol Med 3: 115-119.

Johnson DA, Ludlow JM, Street K, et al. (1987) Double-blind comparison of half-dose and standard-dose flupenthixol decanoate in the maintenance treatment of stabilised out-patients with schizophrenia. Br J Psychiatry 151: 634-638.

Jolley AG, Hirsch SR, Morrison E, et al. (1990) Trial of brief intermittent neuroleptic prophylaxis for selected schizophrenic outpatients: Clinical and social outcome at two years. BMJ 301: 837-842.

Jones C (2018) Alpha7 nicotinic acetylcholine receptor: A potential target in treating cognitive decline in schizophrenia. J Clin Psychopharmacol 38: 247-249.

Jones JC, Day JC, Taylor JR, et al. (1998) Investigation of depot neuroleptic injection site reactions. Psychiatr Bull 22: 605-607.

Jones PB, Barnes TR, Davies L, et al. (2006) Randomized controlled trial of the effect on Quality of Life of second- vs first-generation antipsychotic drugs in schizophrenia: Cost Utility of the Latest Antipsychotic Drugs in Schizophrenia Study (CUtLASS 1). Arch Gen Psychiatry 63: 1079-1087.

Jönsson AK, Spigset O and Hägg S (2012) Venous thromboembolism in recipients of antipsychotics: Incidence, mechanisms and management. CNS Drugs 26: 649-662.

Josiassen RC, Joseph A, Kohegyi E, et al. (2005) Clozapine augmented with risperidone in the treatment of schizophrenia: A randomized, double-blind, placebo-controlled trial. Am J Psychiatry 162: $130-136$.

Joyce EM (2013) Cognitive function in schizophrenia: Insights from intelligence research. Br J Psychiatry 203: 161-162.

Kadra G, Stewart R, Shetty H, et al. (2016) Predictors of long-term ( $\geqslant 6$ months) antipsychotic polypharmacy prescribing in secondary mental healthcare. Schizophr Res 174: 106-112.

Kadra G, Stewart R, Shetty H, et al. (2018) Long-term antipsychotic polypharmacy prescribing in secondary mental health care and the risk of mortality. Acta Psychiatr Scand 138: 123-132.

Kahn RS, Fleischhacker WW, Boter H, et al. (2008) Effectiveness of antipsychotic drugs in first-episode schizophrenia and schizophreniform disorder: An open randomised clinical trial. Lancet 371: 1085-1097.

Kahn RS, Winter van Rossum I, Leucht S, et al. (2018) Amisulpride and olanzapine followed by open-label treatment with clozapine in first-episode schizophrenia and schizophreniform disorder (OPTiMiSE): A three-phase switching study. Lancet Psychiatry 5: 797-807.

Kallen BA and Otterblad OP (2007) Maternal use of selective serotonin re-uptake inhibitors in early pregnancy and infant congenital malformations. Birth Defects Res A Clin Mol Teratol 79: 301-308.

Kam SM, Singh SP and Upthegrove R (2015) What needs to follow early intervention? Predictors of relapse and functional recovery following first-episode psychosis. Early Interv Psychiatry 9: 279-283.

Kamali M, Kelly BD, Clarke M, et al. (2006) A prospective evaluation of adherence to medication in first episode schizophrenia. Eur Psychiatry 21: 29-33.

Kane JM (1992) Clinical efficacy of clozapine in treatment-refractory schizophrenia: An overview. Br J Psychiatry Suppl 17: 41-45.

Kane JM (2008) An evidence-based strategy for remission in schizophrenia. J Clin Psychiatry 69(Suppl 3): 25-30.
Kane JM, Agid O, Baldwin ML, et al. (2019) Clinical guidance on the identification and management of treatment-resistant schizophrenia. J Clin Psychiatry 80: pii: $18 \mathrm{com} 12123$.

Kane JM, Aguglia E, Altamura AC, et al. (1998) Guidelines for depot antipsychotic treatment in schizophrenia. European Neuropsychopharmacology Consensus Conference in Siena, Italy. Eur Neuropsychopharmacol 8: 55-66.

Kane JM, Correll CU, Goff DC, et al. (2009) A multicenter, randomized, double-blind, placebo-controlled, 16-week study of adjunctive aripiprazole for schizophrenia or schizoaffective disorder inadequately treated with quetiapine or risperidone monotherapy. $J$ Clin Psychiatry 70: 1348-1357.

Kane JM, Davis JM, Schooler N, et al. (2002) A multidose study of haloperidol decanoate in the maintenance treatment of schizophrenia. $\mathrm{Am}$ J Psychiatry 159: 554-560.

Kane JM, Jeste DV, Barnes TRE, et al. (1992) Tardive Dyskinesia: A Task Force Report of the American Psychiatric Association. Washington, DC: American Psychiatric Association.

Kane JM, Kishimoto T and Correll CU (2013) Non-adherence to medication in patients with psychotic disorders: Epidemiology, contributing factors and management strategies. World Psychiatry 12: 216-226.

Kane JM, Osuntokun O, Kryzhanovskaya LA, et al. (2009) A 28-week, randomized, double-blind study of olanzapine versus aripiprazole in the treatment of schizophrenia. J Clin Psychiatry 70: 572-581.

Kane JM, Rifkin A, Woerner M, et al. (1985) High dose versus low dose strategies in the treatment of schizophrenia. Psychopharmacol Bull 21: 533-537.

Kane JM, Rifkin A, Woerner M, Reardon G, et al. (1983) Low dose neuroleptic treatment of outpatient schizophrenics. Arch Gen Psychiatry 40: 893-896.

Kantrowitz JT, Swerdlow NR, Dunn W, et al. (2018) Auditory system target engagement during plasticity-based interventions in schizophrenia: A focus on modulation of n-methyl-d-aspartate-type glutamate receptor function. Biol Psychiatry Cogn Neurosci Neuroimaging 3: 581-590.

Kantrowitz JT, Woods SW, Petkova E, et al. (2015) D-serine for the treatment of negative symptoms in individuals at clinical high risk of schizophrenia: A pilot, double-blind, placebo-controlled, randomised parallel group mechanistic proof-of-concept trial. Lancet Psychiatry 2: 403-412.

Kapur S, Zipursky R, Jones C, et al. (2000) Relationship between dopamine $\mathrm{D}(2)$ occupancy, clinical response, and side effects: A doubleblind PET study of first-episode schizophrenia. Am J Psychiatry 157: 514-520.

Karunakaran K, Tungaraza TE and Harborne GC (2007) Is clozapinearipiprazole combination a useful regime in the management of treatment-resistant schizophrenia? J Psychopharmacol 21: 453456.

Karr SJ, Natesan S, McCutcheon R, et al. (2019) Antipsychotics: Mechanisms underlying clinical response and side-effects and novel treatment approaches based on pathophysiology. Neuropharmacology. Epub ahead of print 9 July 2019. doi: 10.1016/j.neuropharm.2019.107704.

Karson C, Duffy RA, Eramo A, et al. (2016) Long-term outcomes of antipsychotic treatment in patients with first-episode schizophrenia: A systematic review. Neuropsychiatr Dis Treat 12: 57-67.

Kay SR, Fiszbein A and Opler LA (1987) The positive and negative syndrome scale (PANSS) for schizophrenia. Schizophr Bull 13: 261-276.

Keck PE, Pope HG and McElroy S (1991) Declining frequency of neuroleptic malignant syndrome in a hospital population. Am J Psychiatry 148: 880-882.

Keefe RS, Bilder RM, Davis SM, et al. (2007) Neurocognitive effects of antipsychotic medications in patients with chronic schizophrenia in the CATIE Trial. Arch Gen Psychiatry 64: 633-647. 
Keefe RS, Meltzer HA, Dgetluck N, et al. (2015) Randomized, doubleblind, placebo-controlled study of encenicline, an alpha7 nicotinic acetylcholine receptor agonist, as a treatment for cognitive impairment in schizophrenia. Neuropsychopharmacology 40: 3053-3060.

Kegeles LS, Abi-Dargham A, Frankle WG, et al. (2010) Increased synaptic dopamine function in associative regions of the striatum in schizophrenia. Arch Gen Psychiatry 67: 231-239.

Kelly DL, Wehring HJ, Earl AK, et al. (2013) Treating symptomatic hyperprolactinemia in women with schizophrenia: Presentation of the ongoing DAAMSEL clinical trial (Dopamine partial Agonist, Aripiprazole, for the Management of Symptomatic ELevated prolactin). BMC Psychiatry 13: 214.

Kem WR, Olincy A, Johnson L, et al. (2018) Pharmacokinetic limitations on effects of an alpha7-nicotinic receptor agonist in schizophrenia: Randomized trial with an extended-release formulation. Neuropsychopharmacology 43: 583-589.

Kemp R, Hayward P, Applewhaite G, et al. (1996) Compliance therapy in psychotic patients: Randomised controlled trial. BMJ 312: 345-349.

Kemp R, Kirov G, Everitt B, et al. (1998) Randomised controlled trial of compliance therapy. 18-month follow-up. Br J Psychiatry 172: 413-419.

Kerwin RW and Bolonna A (2005) Management of clozapine-resistant schizophrenia. Adv Psychiatr Treatment 11: 101-106.

Kesserwani J, Kadra G, Downs J, et al. (2019) Risk of readmission in patients with schizophrenia and schizoaffective disorder newly prescribed clozapine. J Psychopharmacol 33: 449-458.

Kessing LV, Thomsen AF, Mogensen UB, et al. (2010) Treatment with antipsychotics and the risk of diabetes in clinical practice. Br J Psychiatry 197: 266-271.

Khan A, Faucett J, Morrison S, et al. (2013) Comparative mortality risk in adult patients with schizophrenia, depression, bipolar disorder, anxiety disorders, and attention-deficit/hyperactivity disorder participating in psychopharmacology clinical trials. JAMA Psychiatry 70: 1091-1099.

Kim B, Lee SH, Choi TK, et al. (2008) Effectiveness of risperidone long-acting injection in first-episode schizophrenia: In naturalistic setting. Prog Neuropsychopharmacol Biol Psychiatry 32: $123-125$

Kim S-W, Shin I-S, Kim J-M, et al. (2007) Amisulpride versus risperidone in the treatment of depression in patients with schizophrenia: A randomized, open-label, controlled trial. Prog Neuropsychopharmacol Biol Psychiatry 31: 1504-1509.

Kim Y, Wang SM, Kwak KP, et al. (2016) Amisulpride switching in schizophrenic patients who showed suboptimal effect and/ or tolerability to current antipsychotics in a naturalistic setting: An explorative study. Clin Psychopharmacol Neurosci 14: 371-377.

Kimura H, Kanahara N, Sasaki T, et al. (2016) Risperidone long-acting injectable in the treatment of treatment-resistant schizophrenia with dopamine supersensitivity psychosis: Results of a 2-year prospective study, including an additional 1-year follow-up. J Psychopharmacol 30: 795-802.

Kinon BJ, Chen L, Ascher-Svanum H, et al. (2008) Predicting response to atypical antipsychotics based on early response in the treatment of schizophrenia. Schizophr Res 102: 230-240.

Kinon BJ, Chen L, Ascher-Svanum H, et al. (2010) Early response to antipsychotic drug therapy as a clinical marker of subsequent response in the treatment of schizophrenia. Neuropsychopharmacology 35: $581-590$.

Kinon BJ, Kaiser CJ and Ahmed S (2005) Association between early and rapid weight gain and change in weight over one year of olanzapine therapy in patients with schizophrenia and related disorders. J Clin Psychopharmacol 25: 255-258.

Kinon BJ, Kane JM, Johns C, et al. (1993) Treatment of neurolepticresistant schizophrenic relapse. Psychopharm Bull 29: 309-314.
Kinon BJ, Volavka J, Stauffer V, et al. (2008) Standard and higher dose of olanzapine in patients with schizophrenia or schizoaffective disorder: A randomized, double-blind, fixed-dose study. J Clin Psychopharmacol 28: 392-400.

Kirkpatrick B, Fenton WS, Carpenter WT, Jr., et al. (2006) The NIMHMATRICS consensus statement on negative symptoms. Schizophr Bull 32: 214-219.

Kirkpatrick B, Strauss GP, Nguyen L, et al. (2011) The brief negative symptom scale: psychometric properties. Schizophr Bull 37: 300-305.

Kirson NY, Weiden PJ, Yermakov S, et al. (2013) Efficacy and effectiveness of depot versus oral antipsychotics in schizophrenia: Synthesizing results across different research designs. $J$ Clin Psychiatry 74: $568-575$.

Kishi T, Ikuta T, Matsunaga S, et al. (2017a) Comparative efficacy and safety of antipsychotics in the treatment of schizophrenia: A network meta-analysis in a Japanese population. Neuropsychiatr Dis Treat 13: 1281-1302.

Kishi T, Matsuda Y and Iwata N (2017b) Memantine add-on to antipsychotic treatment for residual negative and cognitive symptoms of schizophrenia: A meta-analysis. Psychopharmacology (Berl) 234: 2113-2125.

Kishi T, Oya K and Iwata N (2016) Long-acting injectable antipsychotics for the prevention of relapse in patients with recent-onset psychotic disorders: A systematic review and meta-analysis of randomized controlled trials Psychiatr Res 246: 750-755.

Kishimoto T, De Hert M, Carlson HE, et al. (2012) Osteoporosis and fracture risk in people with schizophrenia. Curr Opin Psychiatry 25: 415-429.

Kishimoto T, Hagi K, Nitta M, et al. (2018) Effectiveness of long-acting injectable vs oral antipsychotics in patients with schizophrenia: A meta-analysis of prospective and retrospective cohort studies. Schizophr Bull 44: 603-619.

Kiviniemi M, Suvisaari J, Koivumaa-Honkanen H, et al. (2013) Antipsychotics and mortality in first-onset schizophrenia: Prospective Finnish register study with 5-year follow-up. Schizophr Res 150: 274-280.

Knapp M, King D, Pugner K, et al. (2004) Non-adherence to antipsychotic medication regimens: Associations with resource use and costs. Br J Psychiatry 184: 509-516.

Knöchel C, Stäblein M, Storchak H, et al. (2014) Multimodal assessments of the hippocampal formation in Schizophrenia and bipolar disorder: Evidences from neurobehavioral measures and functional and structural MRI. NeuroImage Clin 6: 134-144.

Knoph KN, Morgan RJ, 3rd, Palmer BA, et al. (2018) Clozapine-induced cardiomyopathy and myocarditis monitoring: A systematic review. Schizophr Res 199: 17-30.

Kontaxakis VP, Ferentinos PP, Havaki-Kontaxaki BJ, et al. (2005) Randomized controlled augmentation trials in clozapine-resistant schizophrenic patients: a critical review. Eur Psychiatry 20: 409-415.

Kontaxakis VP, Havaki-Kontaxaki BJ, Stamouli SS, et al. (2002) Toxic interaction between risperidone and clozapine: A case report. Prog Neuropsychopharmacol Biol Psychiatry 26: 407-409.

Kotov R, Leong SH, Mojtabai R, et al. (2013) Boundaries of schizoaffective disorder: Revisiting Kraepelin. JAMA Psychiatry 70: 12761286.

Koychev I, Hadjiphilippou S, Lynch J, et al. (2016) Sudden-onset catatonia following clozapine withdrawal: A case report. [letter]. J Clin Psychiatry 77: e899.

Krakowski MI, Czobor P, Citrome L, et al. (2006) Atypical antipsychotic agents in the treatment of violent patients with schizophrenia and schizoaffective disorder. Arch Gen Psychiatry 63: 622-629.

Kreyenbuhl J, Marcus SC, West JC, et al. (2007) Adding or switching antipsychotic medications in treatment-refractory schizophrenia. Psychiatric Serv 58: 983-990.

Kring AM, Gur RE, Blanchard JJ, et al. (2013) The Clinical Assessment Interview for Negative Symptoms (CAINS): Final development and validation. Am J Psychiatry 170: 165-172. 
Krynicki CR, Upthegrove R, Deakin JFW, et al. (2018) The relationship between negative symptoms and depression in schizophrenia: A systematic review. Acta Psychiatr Scand 137: 380-390.

Kulkarni J, Gavrilidis E, Gwini SM, et al. (2016) Effect of adjunctive raloxifene therapy on severity of refractory schizophrenia in women: A randomized clinical trial. JAMA Psychiatry 73: 947-954.

Kumra S, Kranzler H, Gerbino-Rosen G, et al. (2008) Clozapine versus "high-dose" olanzapine in refractory early-onset schizophrenia: An open-label extension study. J Child Adolesc Psychopharmacol 18: 307-316.

Lacro JP, Dunn LB, Dolder CR, et al. (2002) Prevalence of and risk factors for medication nonadherence in patients with schizophrenia: A comprehensive review of recent literature. J Clin Psychiatry 63: 892-909.

Lahti M, Tiihonen J, Wildgust H, et al.(2012) Cardiovascular morbidity, mortality and pharmacotherapy in patients with schizophrenia. Psychol Med 42: 2275-2285.

Lally J, Ajnakina O, Di Forti M, et al. (2016) Two distinct patterns of treatment resistance: Clinical predictors of treatment resistance in first-episode schizophrenia spectrum psychoses. Psychol Med 46: 3231-3240.

Lambert TJ (2007) Switching antipsychotic therapy; what to expect and clinical strategies for improving therapeutic outcomes. J Clin Psychiatry 68 (Suppl 6): 10-13.

Lambert M, Naber D, Schacht A, et al. (2008) Rates and predictors of remission and recovery during 3 years in 392 never-treated patients with schizophrenia. Acta Psychiatr Scand 118: 220-229.

Land R, Siskind D and McArdle P (2017) The impact of clozapine on hospital use: A systematic review and meta-analysis. Acta Psychiatr Scand 135: 296-309.

Lang AE (1994) Withdrawal akathisia: Case reports and a proposed classification of chronic akathisia. Mov Disord 9: 188-192.

Langan J and Shajahan P (2010) Antipsychotic polypharmacy: Review of mechanisms, mortality and management. Psychiatrist 34: 58-62.

Lambiase PD, de Bono JP, Schilling RJ, et al. (2019) British Heart Rhythm Society clinical practice guidelines on the management of patients developing QT prolongation on antipsychotic medication. Arrhythm Electrophysiol Rev 8: 161-165.

Laursen TM, Munk-Olsen T, Nordentoft M, et al. (2007) Increased mortality among patients admitted with major psychiatric disorders: A register-based study comparing mortality in unipolar depressive disorder, bipolar affective disorder, schizoaffective disorder, and schizophrenia. J Clin Psychiatry 68: 899-907.

Lawrence D, Hancock KJ and Kisely S (2013) The gap in life expectancy from preventable physical illness in psychiatric patients in Western Australia: Retrospective analysis of population based registers. $B M J$ 346: $\mathrm{f} 2539$

Lawrence D, Kisely S and Pais J (2010) The epidemiology of excess mortality in people with mental illness. Can J Psychiatry 55: 752760 .

Lawrie SM, Abukmeil SS, Chiswick A, et al. (1997) Qualitative cerebral morphology in schizophrenia: A magnetic resonance imaging study and systematic literature review. Schizophr Res 25: 155-166.

Layland JJ, Liew D and Prior DL (2009) Clozapine-induced cardiotoxicity: A clinical update. Med J Aust 190: 190-192.

Lee HJ, Choi BH, Choi JS, et al. (2019) Effects of cariprazine on hERG $1 \mathrm{~A}$ and hERG 1A/3.1 potassium channels. Eur J Pharmacol 854: 92-100.

Lee SW, Lee JG, Lee BJ, et al. (2007) A 12-week, double-blind, placebocontrolled trial of galantamine adjunctive treatment to conventional antipsychotics for the cognitive impairments in chronic schizophrenia. Int Clin Psychopharmacol 22: 63-68.

Leeson VC, Sharma P, Harrison M, et al. (2011) IQ trajectory, cognitive reserve, and clinical outcome following a first episode of psychosis: A 3-year longitudinal study. Schizophr Bull 37: 768-777.
Legge SE, Hamshere M, Hayes RD, et al. (2016) Reasons for discontinuing clozapine: A cohort study of patients commencing treatment. Schizophr Res 174: 113-119.

Lehman AF, Lieberman JA, Dixon LB, et al. (2004) Practice guideline for the treatment of patients with schizophrenia, second edition. $\mathrm{Am}$ J Psychiatry 161(Suppl): 1-56.

Lehtinen V, Aaltonen J, Koffert T, et al. (2000) Two-year outcome in first-episode psychosis treated according to an integrated model. Is immediate neuroleptisation always needed? Eur Psychiatry 15: 312-320.

Lennox BR, Tomei G, Vincent SA, et al. (2019) Study of immunotherapy in antibody positive psychosis: Feasibility and acceptability (SINAPPS1). J Neurol Neurosurg Psychiatry 90: 365-367.

Leonard CE, Freeman CP, Newcomb CW, et al. (2013) Antipsychotics and the risks of sudden cardiac death and all-cause death: Cohort studies in Medicaid and dually-eligible Medicaid-Medicare beneficiaries of five states. J Clin Exp Cardiolog Suppl 10: 1-9.

Lester H, Marshall M, Jones P, et al. (2011) Views of young people in early intervention services for first-episode psychosis in England. Psychiatr Serv 62: 882-887.

Leucht S, Barnes TRE, Kissling W, et al. (2003) Relapse prevention in schizophrenia with new-generation antipsychotics: A systematic review and exploratory meta-analysis of randomized, controlled trials. Am J Psychiatry 160: 1209-1222.

Leucht S, Busch R and Hamann J (2005) Early onset hypothesis of antipsychotic drug action: A hypothesis tested, confirmed and extended. Biol Psychiatry 57: 1543-1549.

Leucht S, Cipriani A, Spineli L, et al. (2013) Comparative efficacy and tolerability of 15 antipsychotic drugs in schizophrenia: A multipletreatments meta-analysis. Lancet 382: 951-962.

Leucht S, Helfer B, Dold M, et al. (2015) Lithium for schizophrenia. Cochrane Database Syst Rev 10: CD003834.

Leucht S and Heres S (2006) Epidemiology, clinical consequences, and psychosocial treatment of nonadherence in schizophrenia. $J$ Clin Psychiatry 67(Suppl 5): 3-8.

Leucht S, Hierl S, Kissling W, et al. (2012) Putting the efficacy of psychiatric and general medicine medication into perspective: Review of meta-analyses Br J Psychiatry 200: 97-106.

Leucht S and Kane JM (2006) Measurement-based psychiatry: Definitions of response, remission, stability and relapse in schizophrenia. $J$ Clin Psychiatry 67: 1813-1814.

Leucht S, Kane JM, Etschel E, et al. (2006) Linking the PANSS, BPRS and CGI: clinical implications. Neuropsychopharmacology 31: 2316-2325.

Leucht S, Kane JM, Kissling W, et al. (2005) What does the PANSS mean? Schizophr Res 79: 231-238.

Leucht S, Kissling W and McGrath J (2004) Lithium for schizophrenia revisited: a systematic review and meta-analysis of randomized controlled trials. J Clin Psychiatry 65: 177-186.

Leucht S, Leucht C, Huhn M, et al. (2017) Sixty years of placebo-controlled antipsychotic drug trials in acute schizophrenia: Systematic review, Bayesian meta-analysis, and meta-regression of efficacy predictors. Am J Psychiatry 174: 927-942.

Leucht S, Samara M, Heres S, et al. (2014) Dose equivalents for secondgeneration antipsychotics: The minimum effective dose method. Schizophr Bull 40: 314-316.

Leucht S, Tardy M, Komossa K, et al. (2012) Antipsychotic drugs versus placebo for relapse prevention in schizophrenia: A systematic review and meta-analysis. Lancet 379: 2063-2071.

Levinson DF and Simpson GM (1986) Neuroleptic-induced extrapyramidal symptoms with fever. Heterogeneity of the 'neuroleptic malignant syndrome'. Arch Gen Psychiatry 43: 839-848.

Lewis SW, Barnes TRE, Davies L, et al. (2006) Randomized controlled trial of effect of precription of clozapine versus other second-generation antipsychotic drugs in resistant schizophrenia. Schizophr Bull 32: 715-723. 
Li H, Rui Q, Ning X, et al. (2011) A comparative study of paliperidone palmitate and risperidone long-acting injectable therapy in schizophrenia. Prog Neuro-Psychopharmacol Biol Psychiatr 35: 10021008.

Li X, Tang Y and Wang C (2013) Adjunctive aripiprazole versus placebo for antipsychotic-induced hyperprolactinemia: Meta-analysis of randomized controlled trials. PLoS One 8: e70179.

Li XH, Zhong XM, Lu L, et al. (2019) The prevalence of agranulocytosis and related death in clozapine-treated patients: A comprehensive meta-analysis of observational studies. Psychol Med. Epub ahead of print 12 March 2019. doi: 10.1017/S0033291719000369.

Li YY, Zhang YS, Wang J, et al. (2016) Optimal treatment strategies of clozapine for refractory schizophrenia. Zhongguo Yi Xue Ke Xue Yuan Xue Bao 38: 666-678.

Lieberman JA, Dunbar G, Segreti AC, et al. (2013) A randomized exploratory trial of an alpha-7 nicotinic receptor agonist (TC-5619) for cognitive enhancement in schizophrenia. Neuropsychopharmacology 38: 968-975.

Lieberman JA, Koreen AR, Chakos M, et al. (1996) Factors influencing treatment response and outcome of first-episode schizophrenia: Implications for understanding the pathophysiology of schizophrenia. J Clin Psychiatry 57(Suppl 9): 5-9.

Lieberman JA, Phillips M, Gu H, et al. (2003) Atypical and conventional antipsychotic drugs in treatment-naive first-episode schizophrenia: A 52-week randomized trial of clozapine vs chlorpromazine. Neuropsychopharmacol 28: 995-1003.

Lieberman JA, Stroup TS, McEvoy JP, et al. (2005) Effectiveness of antipsychotic drugs in patients with chronic schizophrenia. New Engl J Med 353: 1209-1223.

Lin A, Wood SJ, Nelson B, et al. (2015) Outcomes of non-transitioned cases in a sample at ultra-high risk for psychosis. Am J Psychiatry 172: 249-258.

Lindenmayer JP, Czobor P, Volavka J, et al. (2002) Olanzapine in refractory schizophrenia after failure of typical or atypical antipsychotic treatment: An open-label switch study. J Clin Psychiatry 63: 931935.

Lindenmayer J-P and Kaur A (2016) Antipsychotic management of schizoaffective disorder: A review. Drugs 76: 589-604.

Lindenmayer JP and Kotsaftis A (2000) Use of sodium valproate in violent and aggressive behaviors: A critical review. J Clin Psychiatry 61: $123-128$.

Lingford-Hughes AR, Welch S, Peters L, et al. (2012) BAP updated guidelines: Evidence-based guidelines for the pharmacological management of substance abuse, harmful use, addiction and comorbidity: Recommendations from BAP. J Psychopharmacology 26: 899-952.

Linszen DH, Dingemans PM and Lenior ME (1994) Cannabis abuse and the course of recent-onset schizophrenic disorders. Arch Gen Psychiatry 51: 273-279.

Liu-Seifert H, Osuntokun OO and Feldman PD (2012) Factors associated with adherence to treatment with olanzapine and other atypical antipsychotic medications in patients with schizophrenia. Compr Psychiatry 53: 107-115.

Lopez-Sendon J, Mena MA and Garcia de Yebenes J (2012) Druginduced parkinsonism in the elderly: Incidence, management and prevention. Drugs Aging 29: 105-118.

Lubman D, King JA and Castle DJ (2010) Treating comorbid substance use disorders in schizophrenia. Int Rev Psychiatry 22: 191-201.

Macerollo A and Deuschl G (2018) Deep brain stimulation for tardive syndromes: Systematic review and meta-analysis. J Neurol Sci 389: 55-60.

Macneil CA, Hasty MK, Evans M, et al. (2009) The therapeutic alliance: Is it necessary or sufficient to engender positive outcomes? Acta Neuropsychiatrica 2: 95-98.

Malandain L, Thibaut F, Grimaldi-Bensouda L, et al. (2018) Correlates and predictors of antipsychotic drug polypharmacy in real-life settings: Results from a nationwide cohort study. Schizophr Res 192: 213-218.

Malik S, Lally J, Ajnakina O, et al. (2018) Sodium valproate and clozapine induced neutropenia: A case control study using register data. Schizophr Res 195: 267-273.

Malla A, Chue P, Jordan G, et al. (2016) An exploratory, open-label, randomized trial comparing risperidone long-acting injectable with oral antipsychotic medication in the treatment of early psychosis. Clin Schizophr Rel Psychoses 9: 198-208.

Manu P, Kane JM and Correll CU (2011) Sudden deaths in psychiatric patients. J Clin Psychiatry 72: 936-941.

Marder SR, Alphs L, Anghelescu IG, et al. (2013) Issues and perspectives in designing clinical trials for negative symptoms in schizophrenia. Schizophr Res 150: 328-333.

Marder SR, Van Putten T, Mintz J, et al. (1984) Costs and benefits of two doses of fluphenazine. Arch Gen Psychiatry 41: 1025-1029.

Marder SR, Van Putten T, Mintz J, et al. (1987) Low- and conventionaldose maintenance therapy with fluphenazine decanoate: Two-year outcome. Arch Gen Psychiatry 44: 518-521.

Marder SR and Wirshing DA (2003) Maintenance treatment. In: Hirsch SR and Weinberger DR (eds) Schizophrenia. 2nd ed. Oxford: Blackwell, pp. 474-488.

Marel C, Mills KL, Kingston R, et al. (2016) Guidelines on the Management of Co-occurring Alcohol and Other Drug and Mental Health Conditions in Alcohol and Other Drug Treatment Settings. 2nd ed. Sydney, Australia: Centre of Research Excellence in Mental Health and Substance Use, National Drug and Alcohol Research Centre, University of New South Wales.

Marques TR, Arenovich T, Agid O, et al. (2011) The different trajectories of antipsychotic response: Antipsychotics versus placebo. Psychol Med 41: 1481-1418.

Marques TR, Levine SZ, Reichenberg A, et al. (2014) How antipsychotics impact the different dimensions of schizophrenia: A test of competing hypotheses. Eur Neuropsychopharmacol 24: 1279-1288.

Marshall M, Husain N, Bork N, et al. (2014) Impact of early intervention services on duration of untreated psychosis: Data from the National EDEN prospective cohort study. Schizophr Res 159: 1-6.

Marshall M, Lewis S, Lockwood A, et al. (2005) Association between duration of untreated psychosis and outcome in cohorts of first episode patients: A systematic review. Arch Gen Psychiatry 62: 975 983.

Masand PS and Narasimhan M (2006) Improving adherence to antipsychotic pharmacotherapy. Curr Clin Pharmacol 1: 47-56.

Masand PS, Roca M, Turner MS, et al. (2009) Partial adherence to antipsychotic medication impacts the course of illness in patients with schizophrenia: A review. Prim Care Companion J Clin Psychiatry 11: $147-154$.

Masuda T, Misawa F, Takase M, et al. (2019) Association with hospitalization and all-cause discontinuation among patients with schizophrenia on clozapine vs other oral second-generation antipsychotics: A systematic review and meta-analysis of cohort studies. JAMA Psychiatry. Epub ahead of print 31 July 2019. doi: 10.1001/jamapsychiatry.2019.1702.

May PR, Tuma AH and Dixon WJ (1981) Schizophrenia: A follow-up study of the results of five forms of treatment. Arch Gen Psychiatry 38: 776-784.

Mayoral-van Son J, de la Foz VO, Martinez-Garcia O, et al. (2016) Clinical outcome after antipsychotic treatment discontinuation in functionally recovered first-episode nonaffective psychosis individuals: A 3-year naturalistic follow-up study. J Clin Psychiatry 77: 492-500.

McAllister-Williams RH, Baldwin DS, Cantwell R, et al. (2017) British Association for Psychopharmacology consensus guidance on the use of psychotropic medication preconception, in pregnancy and postpartum 2017. J Psychopharmacol 31: 519-552.

McClave AK, McKnight-Eily LR, Davis SP, et al. (2010). Smoking characteristics of adults with selected lifetime mental illnesses: Results 
from the 2007 National Health Interview Survey. Am J Public Health 100: 2464-2472.

McCreadie RG, Scottish Schizophrenia Lifestyle Group (2003) Diet, smoking and cardiovascular risk in people with schizophrenia: Descriptive study. Br J Psychiatry 183: 534-539.

McCutcheon R, Beck K, Bloomfield MA, et al. (2015) Treatment resistant or resistant to treatment? Antipsychotic plasma levels in patients with poorly controlled psychotic symptoms. J Psychopharmacol 29: 892-897.

McCutcheon R, Beck K, D'Ambrosio E, et al. (2018) Antipsychotic plasma levels in the assessment of poor treatment response in schizophrenia. Acta Psychiatr Scand 137: 39-46.

McEvoy JP, Byerly M, Hamer RM, et al. (2014) Effectiveness of paliperidone palmitate vs haloperidol decanoate for maintenance treatment of schizophrenia: A randomized clinical trial. JAMA 311: 1978-1987.

McEvoy JP, Howe AC and Hogarty GE (1984) Differences in the nature of relapse and subsequent inpatient course between medication compliant and non-compliant schizophrenic patients. J Nerv Ment Dis 172: 412-416.

McEvoy JP, Lieberman JA, Perkins DO, et al. (2007) Efficacy and tolerability of olanzapine, quetiapine, and risperidone in the treatment of early psychosis: A randomized, double-blind 52-week comparison. Am J Psychiatry 164: 1050-1060.

McEvoy JP, Lieberman JA, Stroup TS, et al. (2006) Effectiveness of clozapine versus olanzapine, quetiapine, and risperidone in patients with chronic schizophrenia who did not respond to prior atypical antipsychotic treatment. Am J Psychiatry 163: 600-610.

McGinty J, Haque MS and Upthegrove R (2018) Depression during first episode psychosis and subsequent suicide risk: A systematic review and meta-analysis of longitudinal studies. Schizophr Res 195: 58-66.

McGlashan TH and Carpenter WT Jr (1981) Does attitude toward psychosis relate to outcome? Am J Psychiatry 138: 797-801.

McGlashan TH, Zipursky R, Perkins D, et al. (2006) Randomized, double-blind trial of olanzapine versus placebo in patients prodromally symptomatic for psychosis. Am J Psychiatry 163: 790-799.

McGlashan TH, Zipursky RB, Perkins D, et al. (2003) The PRIME North America randomized double-blind clinical trial of olanzapine versus placebo in patients at risk of being prodromally symptomatic for psychosis. I. Study rationale and design. Schizophr Res 61: 7-18.

McGorry PD, Nelson B, Markulev C, et al. (2017) Effect of omega-3 polyunsaturated fatty acids in young people at ultrahigh risk for psychotic disorders: The NEURAPRO randomized clinical trial. JAMA Psychiatry 74: 19-27.

McGorry PD, Nelson B, Phillips LJ, et al. (2013) Randomized controlled trial of interventions for young people at ultra-high risk of psychosis: Twelve-month outcome. J Clin Psychiatry 74: 349-356.

McGorry P, Yung A, Phillips L, et al. (2002) Randomized controlled trial of interventions designed to reduce the risk of progression to firstepisode psychosis in a clinical sample with subthreshold symptoms. Arch Gen Psychiatry 59: 921-928.

McIntyre RS, Soczynska JK, Woldeyohannes HO, et al. (2007) A preclinical and clinical rationale for quetiapine in mood syndromes. Expert Opin Pharmacother 8: 1211-1219.

Meador K, Reynolds MW, Crean S, et al. (2008) Pregnancy outcomes in women with epilepsy: A systematic review and meta-analysis of published pregnancy registries and cohorts. Epilepsy Res 81: $1-13$.

Medic G, Higashi K, Littlewood KJ, et al. (2013) Dosing frequency and adherence in chronic psychiatric disease: Systematic review and meta-analysis. Neuropsychiatr Dis Treat 9: 119-131.

Medicines and Healthcare products Regulatory Agency (2011) Citalopram and escitalopram: QT interval prolongation. Drug Safety Update.
Available at: www.gov.uk/drug-safety-update/citalopram-andescitalopram-qt-interval-prolongation (accessed September 2016).

Medicines and Healthcare products Regulatory Authority (2018) Valproate use by women and girl.. Available at: https://www.gov.uk/guidance/valproate-use-by-women-and-girls (Last updated 21 June 2019).

Meltzer HY (1992) Dimensions of outcome with clozapine. Br J Psychiatry Suppl 17: 46-53.

Meltzer HY (2015) Pharmacotherapy of cognition in schizophrenia. Current Opin Behav Sci 4: 115-121.

Meltzer HY and Baldessarini RJ (2003) Reducing the risk for suicide in schizophrenia and affective disorders. J Clin Psychiatry 64: 11221129.

Meltzer HY, Bobo WV, Roy A, et al. (2008) A randomized, double-blind comparison of clozapine and high-dose olanzapine in treatment-resistant patients with schizophrenia. J Clin Psychiatry 69: 274-285.

Meltzer HY and Okayli G (1995) Reduction of suicidality during clozapine treatment of neuroleptic-resistant schizophrenia: Impact on risk-benefit assessment. Am J Psychiatry 152: 183-190.

Mentzel TQ, Lieverse R, Bloeman O, et al. (2017) High incidence and prevalence of drug-induced movement disorders in young patients with psychotic disorders. J Clin Psychopharmacol 37: 231-238.

Mentzel TQ, van der Snoek R, Lieverse R, et al. (2018) Clozapine monotherapy as a treatment for antipsychotic-induced tardive dyskinesia: A meta-analysis. J Clin Psychopharmacol 79: Pii: 17r11852.

Menzin J, Boulanger L, Friedman M, et al. (2003) Treatment adherence associated with conventional and atypical antipsychotics in a large state Medicaid program. Psychiatr Serv 54: 719-723.

Meyer N, Gee S, Whiskey E, Taylor D, et al. (2015) Optimising outcomes in clozapine re-challenge following neutropenia: A cohort analysis. J Clin Psychiatry 76: e1410-e1416.

Meyer N, Kerz M, Folarin A, et al. (2018) Capturing rest-activity profiles in schizophrenia using wearable and mobile technologies: Development, implementation, feasibility, and acceptability of a remote monitoring platform. JMIR Mhealth Uhealth 6: e188.

Miklowitz D, O’Brien M, Schlosser D, et al. (2014) Family-focused treatment for adolescents and young adults at high risk for psychosis: Results of a randomized trial. $J$ Am Acad Child Adolesc Psychiatry 53: 848-858.

Milev P, Ho BC, Arndt S, et al. (2005) Predictive values of neurocognition and negative symptoms on functional outcome in schizophrenia: A longitudinal first-episode study with 7-year follow-up. Am J Psychiatry 162: 495-506.

Miller A, Hall CS, Buchanan RW, et al. (2004) The Texas Medication Algorithm Project antipsychotic algorithm for schizophrenia: 2003 update. J Clin Psychiatry 65: 500-508.

Miller BJ and Goldsmith DR (2017) Towards an immunophenotype of schizophrenia: Progress, potential mechanisms, and future directions. Neuropsychopharmacol 42: 299-317.

Miller DD, Caroff SN, Davis SM, et al. (2008) Extrapyramidal sideeffects of antipsychotics in a randomised trial. Br J Psychiatry 193: 279-288.

Miodownik C, Lerner V, Kibari A, et al. (2006) The effect of sudden clozapine discontinuation on management of schizophrenic patients: A retrospective controlled study. J Clin Psychiatry 67: 1204-1208.

Misdrahi D, Tessier A, Husky M, et al. (2018) Evaluation of adherence patterns in schizophrenia using electronic monitoring (MEMS $®$ ): A six-month post-discharge prospective study. Schizophr Res 193: 114-118.

Mitchell AJ, Vancampfort D, Sweers K, et al. (2013) Prevalence of metabolic syndrome and metabolic abnormalities in schizophrenia and related disorders. A systematic review and meta-analysis. Schizophr Bull 39: 306-318. 
Miyamoto S and Fleischhacker WW (2017) The use of long-acting injectable antipsychotics in schizophrenia. Curr Treat Options Psychiatry 4: 117-126.

Mohamed S, Rosenheck RA, Lin H, et al. (2015) Randomized trial of the effect of four second-generation antipsychotics and one first-generation antipsychotic on cigarette smoking, alcohol, and drug use in chronic schizophrenia. J Nerv Ment Dis 203: 486-492.

Molina JD, Lerma-Carrillo I, Leonor M, et al. (2009) Combined treatment with amisulpride in patients with schizophrenia discharged from a short-term hospitalization unit: A 1-year retrospective study. Clin Neuropharmacol 32: 10-15.

Möller H-J (2005) Antidepressive effects of traditional and second generation antipsychotics: A review of the clinical data. Eur Arch Psychiatry Clin Neurosci 255: 83-93.

Monahan J, Vesselinov R, Robbins PC, et al. (2017) Violence to others, violent self-victimization, and violent victimization by others among persons with a mental illness. Psychiatr Serv 68: 516-519.

Montes JM, Medina E, Gomez-Beneyto M, et al. (2012) A short message service (SMS)-based strategy for enhancing adherence to antipsychotic medication in schizophrenia. Psychiatry Res 200: 89-95.

Moore TJ, Furberg CD, Glenmullen J, et al. (2011) Suicidal behavior and depression in smoking cessation treatments. PLoS One 6: e27016.

Morden NE, Lai Z, Goodrich DE, et al. (2012) Eight-year trends of cardiometabolic morbidity and mortality in patients with schizophrenia. Gen Hosp Psychiatry 34: 368-379.

Morgan C, Fearon P, Lappin J, et al. (2017) Ethnicity and long-term course and outcome of psychotic disorders in a UK sample: The ÆSOP-10 study. Br J Psychiatry 211: 88-94.

Morgan C, Lappin J, Heslin M, et al. (2014) Reappraising the long-term course and outcome of psychotic disorders: The AESOP-10 study. Psychol Med 44: 2713-2726.

Morgenstern H and Glazer WM (1993) Identifying risk factors for tardive dyskinesia among long-term outpatients maintained with neuroleptic medications. Arch Gen Psychiatry 50: 723-733.

Morken G, Widen JH and Grawe RW (2008) Non-adherence to antipsychotic medication, relapse and rehospitalisation in recent-onset schizophrenia. BMC Psychiatry 8: 1-7.

Morrison AP, French P, Stewart SL, et al. (2012) Early detection and intervention evaluation for people at risk of psychosis: Multisite randomised controlled trial. $B M J$ 344: e2233.

Morrison AP, French P, Walford L, et al. (2004) Cognitive therapy for the prevention of psychosis in people at ultra-high risk: Randomised controlled trial. Br J Psychiatry 185: 291-297.

Morrison AP, Law H, Carter L, et al. (2018) Antipsychotic drugs versus cognitive behavioural therapy versus a combination of both in people with psychosis: A randomised controlled pilot and feasibility study. Lancet Psychiatry 5: 411-423.

Morrison AP, Turkington D, Pyle M, et al. (2014) Cognitive therapy for people with schizophrenia spectrum disorders not taking antipsychotic drugs: A single-blind randomised controlled trial. Lancet 383 : 1395-403.

Mothi SS, Tandon N, Padmanabhan J, et al. (2015) Increased cardiometabolic dysfunction in first-degree relatives of patients with psychotic disorders. Schizophr Res 165: 103-107.

Mullins CD, Obeidat NA, Cuffel BJ, et al. (2008) Risk of discontinuation of a typical antipsychotic agents in the treatment of schizophrenia. Schizophr Res 98: 8-15.

Murri MB, Guaglianone A, Bugliani M, et al. (2015) Second-generation antipsychotics and neuroleptic malignant syndrome: Systematic review and case report analysis. Drugs $R$ D 15: 45-62.

Muscettola G, Pampallona S, Barbato G, et al. (1993) Persistent tardive dyskinesia: Demographic and pharmacological risk factors. Acta Psychiatr Scand 87: 29-36.

Mustafa FA (2017) Switching away from pipotiazine palmitate: A naturalistic study. Ther Adv Psychopharmacol 7: 25-28.
Nakonezny PA, Byerly MJ and Rush AJ (2008) Electronic monitoring of antipsychotic medication adherence in outpatients with schizophrenia or schizoaffective disorder: An empirical evaluation of its reliability and predictive validity. Psychiatry Res 157: 259-263.

Natesan S, Reckless GE, Nobrega JN, et al. (2006) Dissociation between in vivo occupancy and functional antagonism of dopamine D2 receptors: Comparing aripiprazole to other antipsychotics in animal models. Neuropsychopharmacol 31: 1854-1863.

National Institute for Clinical Excellence (2009) Medicines Concordance and Adherence: Involving Adults and Carers in Decisions about Prescribed Medicines. Available at: www.nice.org.uk.

National Institute for Health and Care Excellence (2011a) Service User Experience in Adult Mental Health Services. Quality standard QS14. Last updated: July 2019. London: National Institute for Health and Care Excellence.

National Institute for Health and Clinical Excellence (2011b) Psychosis with Coexisting Substance Misuse. NICE clinical guideline 120. London: National Institute for Health and Clinical Excellence.

National Institute for Health and Care Excellence (2011c) Alcohol-use Disorders: Diagnosis, Assessment and Management of Harmful Drinking and Alcohol Dependence. Clinical guideline 115. London: National Institute for Health and Care Excellence.

National Institute for Health and Care Excellence (2014a) Psychosis and Schizophrenia in Adults: Prevention and Management. NICE Clinical Guideline 178. London: National Institute for Health and Care Excellence.

National Institute for Health and Care Excellence (2014b) Antenatal and Postnatal Mental Health: Clinical Management and Service Guidance. NICE clinical guideline CG192. London: National Institute for Health and Clinical Excellence.

National Institute for Health and Clinical Excellence (2015) Diabetes in Pregnancy: Management from Preconception to the Postnatal Period. NICE guideline NG3. London: National Institute for Health and Clinical Excellence.

Nederlof AF, Koppenol-Gonzalez GV, Muris P, et al. (2013) Psychiatrists' view on the risk factors for aggressive behavior in psychotic patients. Clin Schizophr Relat Psychoses 7: 131-137.

Nelson B, Amminger GP, Yuen HP, et al. (2018) NEURAPRO: A multicentre RCT of omega-3 polyunsaturated fatty acids versus placebo in young people at ultra-high risk of psychotic disorders-medium-term follow-up and clinical course. NPJ Schizophr 4: 11.

Nelson K and Holmes LB (1989) Malformations due to presumed spontaneous mutations in newborn infants. N Engl J Med 320: 19-23.

Nieuwlaat R, Wilczynski N, Navarro T, et al. (2014) Interventions for enhancing medication adherence. Cochrane Database Systc Rev 11: CD000011.

Nielsen J, Correll CU, Manu P, et al. (2013b) Termination of clozapine treatment due to medical reasons: When is it warranted and how can it be avoided? J Clin Psychiatry 74: 603-613.

Nielsen J, Dahm M, Lublin H, et al. (2010) Psychiatrists' attitude towards and knowledge of clozapine treatment. J Psychopharmacol 24: 965971.

Nielsen J, Graff C, Kanters JK, et al. (2011) Assessing QT interval prolongation and its associated risks with antipsychotics. CNS Drugs 25: 473-490.

Nielsen J, Jensen SO, Friis RB, et al. (2015) Comparative effectiveness of risperidone long-acting injectable vs first-generation antipsychotic long-acting injectables in schizophrenia: Results from a nationwide, retrospective inception cohort study. Schizophr Bull 41: 627-636.

Nielsen J, Young C, Ifteni P, et al. (2016) Worldwide differences in regulations of clozapine use. CNS Drugs 30: 149-161.

Nielsen RE, Levander S, Kjaersdam Telleus G, et al. (2015) Second-generation antipsychotic effect on cognition in patients with schizophrenia - a meta-analysis of randomized clinical trials. Acta Psychiatr Scand 131: 185-196. 
Nielsen RE, Uggerby AS, Jensen SO, et al. (2013a) Increasing mortality gap for patients diagnosed with schizophrenia over the last three decades - a Danish nationwide study from 1980 to 2010. Schizophr Res 146: 22-27.

Nielssen O and Large M (2010) Rates of homicide during the first episode of psychosis and after treatment: A systematic review and metaanalysis. Schizophr Bull 36: 702-712.

Nielssen OB, Malhi GS, McGorry PD, et al. (2012) Overview of violence to self and others during the first episode of psychosis. J Clin Psychiatry 73: e580-e587.

Nikolic N, Kilbride K and Preston P (2019) Clozapine rapid retitration in the community: An assertive approach can prevent admissions. BJPsych Adv 25: 70-71.

Nilsson T and Falk Ö (2015) Rates of violent crimes decrease during periods with antipsychotics and mood stabilisers compared to periods without. Evidence-Based Mental Health 18: 53.

Novick D, Haro JM, Suarez D, et al. (2010) Predictors and clinical consequences of non-adherence with antipsychotic medication in the outpatient treatment of schizophrenia. Psychiatry Res 176: 109-113.

Nuechterlein KH, Subotnik KL, Green MF, et al. (2011) Neurocognitive predictors of work outcome in recent-onset schizophrenia. Schizophr Bull 37(Suppl 2): S33-S40.

Nutt DJ and Blier P (2016) Neuroscience-based Nomenclature (NbN) for Journal of Psychopharmacology. J Psychopharmacol 30: 413415.

Nyberg S, Farde L, Halldin C, et al. (1995) D2 dopamine receptor occupancy during low-dose treatment with haloperidol decanoate. Am J Psychiatry 152: 173-178.

O'Donnell C, Donohoe G, Sharkey L, et al. (2003) Compliance therapy: A randomised controlled trial in schizophrenia. BMJ 327: 834.

Ohmori T, Ito K, Abekawa T, et al. (1999) Psychotic relapse and maintenance therapy in paranoid schizophrenia: A 15 year follow up. Eur Arch Psychiatry Clin Neurosci 249: 73-78.

Okhuijsen-Pfeifer C, Huijsman EAH, Hasan A, et al. (2018) Clozapine as a first- or second-line treatment in schizophrenia: A systematic review and meta-analysis. Acta Psychiatr Scand 138: 281-288.

Olfson M, Marcus SC and Ascher-Svanum H (2007) Treatment of schizophrenia with long-acting fluphenazine, haloperidol, or risperidone. Schizophr Bull 33: 1379-1387.

Olfson M, Mechanic D, Boyer CA, et al. (1999) Assessing clinical predictions of early rehospitalization in schizophrenia. J Nerv Ment Dis 187: 721-729.

Olivares JM, Sermon J, Hemels M, et al. (2013) Definitions and drivers of relapse in patients with schizophrenia: A systematic literature review. Ann Gen Psychiatry 12: 32.

Omachi Y and Sumiyoshi T (2018) Dose reduction/discontinuation of antipsychotic drugs in psychosis; effect on cognition and functional outcomes. Front Psychiatry 9: 447.

Ortiz-Orendain J, Castiello-de Obeso S, Colunga-Lozano LE, et al. (2018) Antipsychotic combinations for schizophrenia. Schizoph Bull 44: $15-17$.

Ortiz-Orendain J, Castiello-de Obeso S, Colunga-Lozano LE, et al. (2017) Antipsychotic combinations for schizophrenia. Cochrane Database Syst Rev 6: CD009005.

Osborn DP, Levy G, Nazareth I, et al. (2007) Relative risk of cardiovascular and cancer mortality in people with severe mental illness from the United Kingdom's General Practice Research Database. Arch Gen Psychiatry 64: 242-249.

Overall JE and Gorham DR (1962) The brief psychiatric rating Scale. Psychol Rep 10: 799-812.

Owens DGC (2014) A Guide to the Extrapyramidal Side-Effects of Antipsychotic Drugs. 2nd ed. Cambridge: Cambridge University Press.

Owens DGC (2019a) Tardive dyskinesia update: The syndrome. BJPsych $A d v$ 25: 57-69.

Owens DGC (2019b) Tardive dyskinesia update: Treatment and management. BJPsych Adv 25: 78-89.
Padmanabhan JL, Nanda P, Tandon N, et al. (2016) Polygenic risk for type 2 diabetes mellitus among individuals with psychosis and thier relatives. J Psychiatr Res 77: 52-58.

Palmer BA, Pankratz VS and Bostwick JM (2005) The lifetime risk of suicide in schizophrenia: A reexamination. Arch Gen Psychiatry 62: $247-253$.

Palmsten K, Hernández-Díaz S, Huybrechts KF, et al. (2013) Use of antidepressants near delivery and risk of postpartum haemorrhage: Cohort study of low income women in the United States. BMJ 347: f4877.

Pandina G, Lane R, Gopal S, et al. (2011) A double-blind study of paliperidone palmitate and risperidone long-acting injectable in adults with schizophrenia. Prog Neuro-Psychopharmacol Biol Psychiatry 35: $218-226$.

Panizzutti R, Fisher M, Garrett C, et al. (2019) Association between increased serum D-serine and cognitive gains induced by intensive cognitive training in schizophrenia. Schizophr Res 207: 63-69.

Parellada E and Bioque M (2016) Barriers to the use of long-acting injectable antipsychotics in the management of schizophrenia. CNS Drugs 30: 689-701.

Parikh T, Goyal D, Scarff JR, et al. (2014) Antipsychotic drugs and safety concerns for breast-feeding infants. South Med J 107: 686-688.

Patchan K, Vyas G, Hackman AL, et al. (2018) Clozapine in reducing aggression and violence in forensic populations. Psychiatr $Q$ 89: 157-168.

Patel MX, Arista IA, Taylor M, et al. (2013) How to compare doses of different antipsychotics: A systematic review of methods. Schizophr Res 149: 141-148.

Patel MX and David AS (2005) Why aren't depot antipsychotics prescribed more often and what can be done about it? Adv Psychiatr Treat 11: 203-211.

Patel MX, De Zoysa N, Bernadt M, et al. (2009) Depot and oral antipsychotics: Patient preferences and attitudes are not the same thing. $J$ Psychopharmacol 23: 789-96.

Paton C, Barnes TRE, Cavanagh MR, et al. (2008) High-dose and combination antipsychotic prescribing in acute adult wards in the UK: the challenges posed by p.r.n. prescribing. Br J Psychiatry 192: 435-439.

Paton C, Whittington C and Barnes TR (2007) Augmentation with a second antipsychotic in patients with schizophrenia who partially respond to clozapine: A meta-analysis. J Clin Psychopharmacol 27: 198-204.

Patall EA, Cooper H and Robinson JC (2008) The effects of choice on intrinsic motivation and related outcomes: A meta-analysis of research findings. Psychol Bull 134: 270-300.

Peng M, Gao K, Ding Y, et al. (2013) Effects of prenatal exposure to atypical antipsychotics on postnatal development and growth of infants: A case-controlled, prospective study. Psychopharmacology (Berl) 228: 577-584.

Perkins DO, Gu H, Weiden PJ, et al. (2008) Comparison of atypicals in first episode study group. Predictors of treatment discontinuation and medication non-adherence in patients recovering from a first episode of schizophrenia, schizophreniform disorder, or schizo-affective disorder: A randomised, double-blind, flexible-dose, multicentre study. J Clin Psychiatry 69: 106-113.

Perry BI, McIntosh G, Weich S, et al. (2016) The association between first-episode psychosis and abnormal glycaemic control, systematic review and meta-analysis. Lancet Psychiatry 3: 1049-1058.

Petersen I, McCrea RL, Sammon CJ, et al. (2016) Risks and benefits of psychotropic medication in pregnancy: Cohort studies based on UK electronic primary health care records. Health Technol Assess 20: $1-176$.

Petrakis IL, Nich C and Ralevski E (2006) Psychotic spectrum disorders and alcohol abuse: A review of pharmacotherapeutic strategies and a report on the effectiveness of naltrexone and disulfiram. Schizophr Bull 32: 644-654.

Pharoah F, Mari J, Rathbone J, et al. (2010) Family intervention for schizophrenia. Cochrane Database Syst Rev 12: CD000088. 
Piette JD, Heisler M, Ganoczy D, et al. (2007) Differential medication adherence among patients with schizophrenia and comorbid diabetes and hypertension. Psychiatric Serv 58: 207-212.

Pileggi DJ and Cook AM (2016) Neuroleptic malignant syndrome: Focus on treatment and rechallenge. Ann Pharmacother 50: 973-981.

Pillinger T, Beck K, Gobjila C, et al. (2017) Impaired glucose homeostasis in first-episode schizophrenia, a systematic review and metaanalysis. JAMA Psychiatry 74: 261-269.

Plavén-Sigray P, Matheson GJ, Collste K, et al. (2018) Positron emission tomography studies of the glial cell marker translocator protein in patients with psychosis: A meta-analysis using individual participant data. Biol Psychiatry 84: 433-442.

Pollack TA, McCormack R, Peakman M, et al. (2014) Prevalence of antiN-methyl-D-aspartate (NMDA) receptor antibodies in patients with schizophrenia and related psychoses: A systematic review and metaanalysis. Psychol Med 44: 2475-2487.

Porcelli S, Balzarro B and Serretti A (2012) Clozapine resistance: Augmentation strategies. Eur Neuropsychopharmacol 22: 165-182.

Preskorn SH, Gawryl M, Dgetluck N, et al. (2014) Normalizing effects of EVP-6124, an alpha-7 nicotinic partial agonist, on event-related potentials and cognition: A proof of concept, randomized trial in patients with schizophrenia. J Psychiatr Pract 20: 12-24.

Priebe S, Yeeles K, Bremner S, et al. (2013) Effectiveness of financial incentives to improve adherence to maintenance treatment with antipsychotics: a cluster randomised controlled trial. BMJ 347: f5847.

Priebe S, Bremner SA and Pavlickova H (2016) Discounting financial incentives for adherence to antipsychotic depot medication: longterm outcomes of a cluster randomised controlled trial. BMJ Open 6: $\mathrm{e} 011673$.

Pringsheim T, Gardner D, Addington D, et al. (2018) The assessment and treatment of antipsychotic-induced akathisia. Can J Psychiatry 63: 719-729.

Prudo R and Blum HM (1987) Five-year outcome and prognosis in schizophrenia: A report from the London Field Research Centre of the International Pilot Study of Schizophrenia. Br J Psychiatry150: $345-354$.

Purcell SM, Wray NR, Stone JL, et al. (2009) Common polygenic variation contributes to risk of schizophrenia and bipolar disorder. Nature 460: 748-752.

Qin P and Nordentoft M (2005) Suicide risk in relation to psychiatric hospitalization: Evidence based on longitudinal registers. Arch Gen Psychiatry 62: 427-432.

Rabinovitch M, Béchard-Evans L, Schmitz N, et al. (2009) Early predictors of nonadherence to antipsychotic therapy in first-episode psychosis. Can J Psychiatry 54: 28-35.

Rathod S, Kingdon D, Smith P, et al. (2005) Insight into schizophrenia: The effects of cognitive behavioural therapy on the components of insight and association with sociodemographics - data on a previously published randomised controlled trial. Schizophr Res 74: 211-219.

Ray WA, Chung CP, Murray KT, et al. (2009) Atypical antipsychotic drugs and the risk of sudden cardiac death. N Engl J Med 360: 225-235.

Reilly JG, Ayis SA, Ferrier IN, et al. (2002) Thioridazine and sudden unexplained death in psychiatric in-patients. $B r J$ Psychiatry 180: $515-522$.

Reine G, Lancon C, Di Tucci S, et al. (2003) Depression and subjective quality of life in chronic phase schizophrenic patients. Acta Psychiatr Scand 108: 297-303.

Remington G, Addington D, Honer W, et al. (2017) Guidelines for the pharmacotherapy of schizophrenia in adults. Can J Psychiatry 62: 604-616.

Remington G, Chue P, Stip E, et al. (2005) The crossover approach to switching antipsychotics: What is the evidence? Schizophr Res 76: 267-272.

Reynolds GP and McGowan O (2017) Mechanisms underlying metabolic disturbances associated with psychosis and antipsychotic drug treatment. J Psychopharmacol 31: 1430-1436.
Ricciardi L, Pringsheim T, Barnes TRE, et al. (2019) Treatment recommendations for tardive dyskinesia. Can J Psychiatry 64: 388-399.

Richardson K, Fox C, Maidment I, et al. (2018) Anticholinergic drugs and risk of dementia: Case-control study. BMJ 361: k1315.

Richardson K, Schoen M, French B, et al. (2013) Statins and cognitive function: A systematic review. Ann Intern Med 159: 688-697.

Rickards H, Jacob S, Lennox B, et al. (2014) Autoimmune encephalitis: A potentially treatable cause of mental disorder. Adv Psychiatr Treat 20: $92-100$.

Ringback Weitoft G, Berglund M, Lindstrom EA, et al. (2014) Mortality, attempted suicide, re-hospitalisation and prescription refill for clozapine and other antipsychotics in Sweden-a register-based study. Pharmacoepidemiol Drug Saf 23: 290-298.

Ringen PA, Engh JA, Birkenaes AB, et al. (2014) Increased mortality in schizophrenia due to cardiovascular disease - a non-systematic review of epidemiology, possible causes, and interventions. Front Psychiatry 5: 137.

Robert LW and Geppert CMA (2004) Ethical use of long-acting medications in the treatment of severe and persistent mental illness. Compr Psychiatry 45: 161-167.

Roberts E, Eden Evins A, McNeill A, et al. (2016) Efficacy and tolerability of pharmacotherapy for smoking cessation in adults with serious mental illness: A systematic review and network meta-analysis. Addiction 111: 599-612.

Robinson D, Woerner MG, Alvir JMJ, et al. (1999) Predictors of relapse following response from a first episode of schizophrenia or schizoaffective disorder. Arch Gen Psychiatry 56: 241-247.

Rochester MP, Kane AM, Linnebur SA, et al. (2018) Evaluating the risk of QTc prolongation associated with antidepressant use in older adults: A review of the evidence. Ther Adv Drug Saf 9: 297-308.

Rosebush P, Stewart T and Gelenberg A (1989) Twenty neuroleptic rechallenges after neuroleptic malignant syndrome in 15 patients. $J$ Clin Psychiatry 50: 295-298.

Rosenheck R, Chang S, Choe Y, et al. (2000) Medication continuation and compliance: A comparison of patients treated with clozapine and haloperidol. J Clin Psychiatry 61: 382-386.

Rosenheck RA, Davis S, Covell N, et al. (2009) Does switching to a new antipsychotic improve outcomes? Data from the CATIE Trial. Schizophr Res 107: 22-29.

Ross LE, Grigoriadis S, Mamisashvili L, et al. (2013) Selected pregnancy and delivery outcomes after exposure to antidepressant medication: A systematic review and meta-analysis. JAMA Psychiatry 70: 436-443.

Ross RG and Freedman R (2015) Endophenotypes in schizophrenia for the perinatal period: Criteria for validation. Schizophr Bull 41: 824-834.

Rostami-Hodjegan A, Amin AM, Spencer EP, et al. (2004) Influence of dose, cigarette smoking, age, sex, and metabolic activity on plasma clozapine concentrations: A predictive model and nomograms to aid clozapine dose adjustment and to assess compliance in individual patients. J Clin Psychopharmacol 24: 70-78.

Roth BL (2008) High-dose olanzapine for treatment-resistant schizophrenia. J Clin Psychiatry 69: 176-177.

Royal College of Psychiatrists (2014) Consensus statement on high-dose antipsychotic medication. College Report CR190, 2014.

Royal College of Psychiatrists (2018) National Clinical Audit of Psychosis - National Report for the Core Audit 2018. London: Healthcare Quality Improvement Partnership. Available at: https:// www.rcpsych.ac.uk/docs/default-source/improving-care/ccqi/ national-clinical-audits/ncap-library/ncap-national-report-for-coreaudit-2018.pdf?sfvrsn $=23$ c6a262 2 .

Rubio JM, Taipale H, Correll CU, et al. (2019) Psychosis breakthrough on antipsychotic maintenance: Results from a nationwide study. Psychol Med. Epub ahead of print 13 June 2019. doi: 10.1017/ S0033291719001296.

Ruhrmann S (2007) Acute effects of treatment for prodromal symptoms for people putatively in a late initial prodromal state of psychosis. $\mathrm{Br}$ J Psychiatry 191: S88-S95. 
Rummel-Kluge C, Komossa K, Schwarz S, et al. (2010) Head-to-head comparisons of metabolic side effects of second generation antipsychotics in the treatment of schizophrenia: A systematic review and meta-analysis. Schizophr Res 123: 225-233.

Sachdev P and Kruk J (1994) Clinical characteristics and predisposing factors in acute drug-induced akathisia. Arch Gen Psychiatry 51: 963-974.

Saha S, Chant D and McGrath J (2007) A systematic review of mortality in schizophrenia: Is the differential mortality gap worsening over time? Arch Gen Psychiatry 64: 1123-1131.

Salgueiro M and Segarra R (2019) Long-acting injectable second-generation antipsychotics in first-episode psychosis: A narrative review. Int Clin Psychopharmacology 34: 51-56.

Salimi K, Jarskog LF and Lieberman JA (2009) Antipsychotic drugs for first-episode schizophrenia: A comparative review. CNS Drugs 23: 837-855

Salvo F, Pariente A, Shakir S, et al. (2016) Sudden cardiac and sudden unexpected death related to antipsychotics: A meta-analysis of observational studies. Clin Pharmacol Ther 99: 306-314.

Samara MT, Dold M, Gianatsi M, et al. (2016) Efficacy, acceptability, and tolerability of antipsychotics in treatment-resistant schizophrenia: A network meta-analysis. JAMA Psychiatry 73: 199-210.

Samara MT, Klupp E, Helfer B, et al. (2018) Increasing antipsychotic dose versus switching antipsychotic for non-response in schizophrenia. Cochrane Database Syst Rev 5: CD011884.

Samara MT, Leucht C, Leeflang MM, et al. (2015) Early improvement as a predictor of later response to antipsychotics in schizophrenia: A diagnostic test review. Am J Psychiatry 172: 617-629.

Sampson S, Joshi K, Mansour M, et al. (2013) Intermittent drug techniques for schizophrenia. Schizophr Bull 39: 960-961.

Sandhu A, Ives J, Birchwood M, et al. (2013) The subjective experience and phenomenology of depression following first episode psychosis: A qualitative study using photo-elicitation. $J$ Affect Disord 149: 166-174.

Santos B, Gonzalez-Fraile E, Zabala A, et al. (2018) Cognitive improvement of acetylcholinesterase inhibitors in schizophrenia. $J$ Psychopharmacol 32: 1155-1166.

Sanz-Fuentenebro J, Taboada D, Paloma T, et al. (2013) Randomized trial of clozapine vs. risperidone in treatment-naive first-episode schizophrenia: Results after one year. Schizophr Res 149: 156-161.

Sara GE, Large MM, Matheson SL, et al. (2015) Stimulant use disorders in people with psychosis: A meta-analysis of rate and factors affecting variation. Aust N Z J Psychiatry 49: 106-117.

Satterthwaite TD, Wolf DH, Rosenheck RA, et al. (2008) A meta-analysis of the risk of acute extrapyramidal symptoms with intramuscular antipsychotics for the treatment of agitation. J Clinic Psychiatry 69: 1869-1879.

Saunders KE and Hawton K (2009) The role of psychopharmacology in suicide prevention. Epidemiol Psichiatr Soc 18: 172-178.

Schimmelmann BG, Conus P, Edwards J, et al. (2005) Diagnostic stability 18 months after treatment initiation for first-episode psychosis. $J$ Clin Psychiatry 66: 1239-1246.

Schneider-Thoma J, Efthimiou O, Huhn M, et al. (2018) Second-geneneration antipsychotic drugs and short-term mortality: A systematic review and meta-analysis of placebo-controlled randomised controlled trials. Lancet Psychiatry 5: 653-663.

Schnell T, Koethe D, Krasnianski A, et al. (2014) Ziprasidone versus clozapine in the treatment of dually diagnosed (DD) patients with schizophrenia and cannabis use disorders: A randomized study. Am J Addict 23: 308-312.

Schooler NR, Keith SJ, Severe JB, et al. (1997) Relapse and rehospitalization during maintenance treatment of schizophrenia. Effects of dose reduction and family treatment. Arch Gen Psychiatry 54: 453-463.

Schooler N, Rabinowitz J, Davidson M, et al. (2005) Risperidone and haloperidol in first episode psychosis: A long-term randomized trial. Am J Psychiatry 162: 947-953.
Schreiner A, Aadamsoo K, Altamura AC, et al. (2015) Paliperidone palmitate versus oral antipsychotics in recently diagnosed schizophrenia. Schizophr Res 169: 393-399.

Schreiner A, Caspi A, Bergmans P, et al. (2017) Switching from oral atypical antipsychotic monotherapy to paliperidone palmitate once-monthly in non-acute patients with schizophrenia: A prospective, open-label, interventional study. Psychopharmacology (Berl) 234: 3-13.

Schubert MH, Young KA and Hicks PB (2006) Galantamine improves cognition in schizophrenic patients stabilized on risperidone. Biol Psychiatry 60: 530-563.

Schulte P (2003) What is an adequate trial with clozapine?: Therapeutic drug monitoring and time to response in treatment-refractory schizophrenia. Clin Pharmacokinet 42: 607-618.

Schulte P (2006) Risk of clozapine-associated agranulocytosis and mandatory white blood cell monitoring. Ann Pharmacother 40: 683-688.

Schultze-Lutter F, Addington J, Ruhrmann S, et al. (2007) Giovanni Fioriti Editore. Rome, Italy. Schizophrenia Proneness Instrument: Adult Version (SPI-A).

Schwartz JE, Fennig S, Tanenberg-Karant M, et al. (2000) Congruence of diagnoses 2 years after a first-admission diagnosis of psychosis. Arch Gen Psychiatry 57: 593-600.

Scott J, Leboyer M, Hickie I, et al. (2013) Clinical staging in psychiatry: A cross-cutting model of diagnosis with heuristic and practical value. Br J Psychiatry 202: 243-245.

Scott J, Marwaha S, Ratheesh A, et al. (2016) Bipolar at-risk criteria: An examination of which clinical features have optimal utility for identifying youth at risk of early transition from depression to bipolar disorders. Schizophr Bull 43: 737-744.

Sendt KV, Tracy DK and Bhattacharyya S (2015) A systematic review of factors influencing adherence to antipsychotic medication in schizophrenia-spectrum disorders. Psychiatry Res 225: 14-30.

Sepehrmanesh Z, Heidary M, Akasheh N, et al. (2018) Therapeutic effect of adjunctive $\mathrm{N}$-acetyl cysteine (NAC) on symptoms of chronic schizophrenia: A double-blind, randomized clinical trial. Prog Neuropsychopharmacol Biol Psychiatry 82: 289-296.

Sevy S, Robinson DG, Sunday S, et al. (2011) Olanzapine vs. risperidone in patients with first-episode schizophrenia and a lifetime history of cannabis use disorders: 16-week clinical and substance use outcomes. Psychiatry Res 188: 310-314.

Shah P, Iwata Y, Plitman E, et al. (2018) The impact of delay in clozapine initiation on treatment outcomes in patients with treatment-resistant schizophrenia: A systematic review. Psychiatry Res 268: 114-122.

Shakespeare J and Sisodyia S (2019) Guidance Document on Valproate use in Women and Girls of Childbearing Years. Royal College of General Practitioners and Association of British Neurologists and Royal College of Physicians, March 2019. Available at: https:// www.rcog.org.uk/globalassets/documents/guidelines/valproateguidance-march-2019.pdf

Shekelle PG, Woolf SH, Eccles M, et al. (1999) Clinical guidelines: Developing guidelines. BMJ 318: 593-596.

Shen ZQ, Gao SY, Li SX, et al. (2017) Sertraline use in the first trimester and risk of congenital anomalies. A systematic review and metaanalysis of cohort studies. Br J Clin Pharmacol 83: 909-922.

Shiloh R, Zemishlany Z, Aizenberg D, et al. (1997) Sulpiride augmentation in people with schizophrenia partially responsive to clozapine. A double-blind, placebo-controlled study. Br J Psychiatry 171: 569-573.

Shim JC, Jung DU, Jung SS, et al. (2012) Adjunctive varenicline treatment with antipsychotic medications for cognitive impairments in people with schizophrenia: A randomized double-blind placebo-controlled trial. Neuropsychopharmacology 37: 660-668.

Shin H-W and Chung SJ (2012) Drug-induced parkinsonism. J Clinical Neurol 8: 15-21.

Shiovitz TM, Welke TL, Tigel PD, et al. (1996) Cholinergic rebound and rapid onset psychosis following abrupt clozapine withdrawal. Schizophr Bull 22: 591-595. 
Shulman M, Miller A, Misher J, et al. (2014) Managing cardiovascular disease risk in patients treated with antipsychotics: A multidisciplinary approach. J Multidiscip Healthc 7: 489-501.

Sicouri S and Antzelevitch C (2018) Mechanisms underlying the actions of antidepressant and antipsychotic drugs that cause sudden cardiac arrest. Arrhythm Electrophysiol Rev 7: 199-209.

Sikich L, Frazier JA, McClellan J, et al. (2008) Double-blind comparison of first- and second-generation antipsychotics in early-onset schizophrenia and schizo-affective disorder: Findings from the treatment of early-onset schizophrenia spectrum disorders (TEOSS) study. Am J Psychiatry 165: 1420-1431.

Silvestri S, Seeman MV, Negrete JC, et al. (2000) Increased dopamine D2 receptor binding after long-term treatment with antipsychotics in humans: A clinical PET study. Psychopharmacology (Berl) 152: 174-180.

Sinclair JMA, Chambers SE, Shiles CJ, et al. (2016) Safety and tolerability of pharmacological treatment of alcohol dependence: Comprehensive review of evidence. Drug Safety 39: 267-245.

Singh H, Levinson DF, Simpson GM, et al. (1990) Acute dystonia during fixed-dose neuroleptic treatment. J Clin Psychopharmacol 10: 389-396.

Siris SG (2000) Depression in schizophrenia: Perspective in the era of “Atypical" antipsychotic agents. Am J Psychiatry 157: 1379-1389.

Siskind D, McCartney L, Goldschlager R, et al. (2016) Clozapine v. firstand second-generation antipsychotics in treatment-refractory schizophrenia: Systematic review and meta-analysis. Br J Psychiatry 209: 385-392.

Siskind D, Reddel T, MacCabe JH, et al. (2019) The impact of clozapine initiation and cessation on psychiatric hospital admissions and bed days: A mirror image cohort study. Psychopharmacology (Berl) 236: 1931-1935.

Siskind D, Siskind V and Kisely S (2017) Clozapine response rates among people with treatment-resistant schizophrenia: Data from a systematic review and meta-analysis. Can J Psychiatry 62: 772-777.

Smeraldi E, Cavallaro R, Folnegović-Šmalc V, et al. (2013) Long-term remission in schizophrenia and Schizoaffective disorder: Results from the risperidone long-acting injectable versus quetiapine relapse prevention trial (ConstaTRE). Ther Adv Psychopharmacol 3: 191-199.

Smith DJ, Langan J, McLean G, et al. (2013) Schizophrenia is associated with excess multiple physical-health comorbidities but low levels of recorded cardiovascular disease in primary care: Cross-sectional study. BMJ Open 3: pii: e002808.

Smith RC, Amiaz R, Si TM, et al. (2016) Varenicline effects on smoking, cognition, and psychiatric symptoms in schizophrenia: A doubleblind randomized trial. PLoS One 11: e0143490.

Smith TE, Weston CA and Lieberman JA (2009) Schizophrenia (maintenance treatment). Clin Evid (Online) pii: 1007.

Sneider B, Pristed SG, Correll CU, et al. (2015) Frequency and correlates of antipsychotic polypharmacy among patients with schizophrenia in Denmark: A nation-wide pharmacoepidemiological study. Eur Neuropsychopharmacol 25: 1669-1676.

Sommer IE, Begemann MJ, Temmerman A, et al. (2012) Pharmacological augmentation strategies for schizophrenia patients with insufficient response to clozapine: A quantitative literature review. Schizophr Bull 38: 1003-1011.

Sommer IE, de Kort GA, Meijering AL, et al. (2013) How frequent are radiological abnormalities in patients with psychosis? A review of 1379 MRI scans. Schizophr Bull 39: 815-819.

Sommer IE, van Westrhenen R, Begeman MJH, et al. (2014) Efficacy of anti-inflammatory agents to improve symptoms in patients with schizophrenia: An update. Schizophr Bull 40: 181-191.

Souza JS, Kayo M, Tassell I, et al. (2013) Efficacy of olanzapine in comparison with clozapine for treatment-resistant schizophrenia: Evidence from a systematic review and meta-analyses. CNS Spectr 18: 82-89.
Sparshatt A, Taylor D, Patel MX, et al. (2010) A systematic review of aripirazole. J Clin Psychiatry 71: 1447-1456.

Speyer H, Christian Brix Nørgaard H, Birk M, et al. (2016) The CHANGE trial: No superiority of lifestyle coaching plus care coordination plus treatment as usual compared to treatment as usual alone in reducing risk of cardiovascular disease in adults with schizophrenia spectrum disorders and abdominal obesity. World Psychiatry 15: 155-165.

Stauffer VL, Case M, Kinon BJ, et al. (2011) Early response to antipsychotic therapy as a clinical marker of subsequent response in the treatment of patients with first-episode psychosis. Psychiatry Res 187: $42-48$.

Stegmayer K, Walther S and van Harten P (2018) Tardive dyskinesia associated with atypical antipsychotics: Prevalence, mechanisms and management strategies. CNS Drugs 2: 135-147.

Stephen PJ and Williamson J (1984) Drug-induced parkinsonism in the elderly. Lancet 2: 1082-1083.

Stephenson JJ, Tunceli O, Gu T, et al. (2012) Adherence to oral secondgeneration antipsychotic medications in patients with schizophrenia and bipolar disorder: Physicians' perceptions of adherence vs. pharmacy claims. Int J Clin Pract 66: 565-573.

Staring AB, Van der Gaag M, Van den Berge M, et al. (2009) Stigma moderates the associations of insight with depressed mood, low selfesteem, and low quality of life in patients with schizophrenia spectrum disorders. Schizophr Res 115: 363-369.

Stone-Brown K, Naji M, Francioni A, et al. (2016) Psychotropic prescribing in seriously violent men with schizophrenia or personality disorder in a UK high security hospital. CNS Spectr 2: 60-69.

Stone JM, Roux S, Taylor D, et al. (2018) First-generation versus second-generation long-acting injectable antipsychotic drugs and time to relapse. Ther Adv Psychopharmacol 8: 333-336.

Straus SM, Bleumink GS, Dieleman JP, et al. (2004) Antipsychotics and the risk of sudden cardiac death. Arch Intern Med 164: 1293-1297.

Strawn JR, Keck PE and Caroff SN (2007) Neuroleptic malignant syndrome. Am J Psychiatry 164: 870-876.

Suarez D and Haro JM (2008) Overview of the findings from the European SOHO study. Expert Rev Neurother 8: 873-880.

Subotnik KL, Casaus LR, Ventura J, et al. (2015) Long-acting injectable risperidone for relapse prevention and control of breakthrough symptoms after a recent first episode of schizophrenia. A randomized clinical trial. JAMA Psychiatry 72: 822-829.

Sutterland AL, Blom MT, Ladee K, et al. (2019) Increased prevalence of ECG suspicious for Brugada Syndrome in recent onset schizophrenia spectrum disorders. Schizophr Res 210: 59-65.

Suzuki T, Uchida H, Watanabe K, et al. (2007) How effective is it to sequentially switch among olanzapine, quetiapine and risperidone? A randomized, open-label study of algorithm-based antipsychotic treatment to patients with symptomatic schizophrenia in the realworld clinical setting. Psychopharmacology (Berl) 195: 285-295.

Swartz MS, Wagner HR, Swanson JW, et al. (2006) Substance use in persons with schizophrenia: Baseline prevalence and correlates from the NIMH CATIE study. J Nerv Ment Dis 194: 164-172.

Swartz MS, Wagner HR, Swanson JW, et al. (2008) The effectiveness of antipsychotic medications in patients who use or avoid illicit substances: Results from the CATIE study. Schizophr Res 100: $39-52$.

Swerdlow NR, Bhakta S, Chou HH, et al. (2016) Memantine effects on sensorimotor gating and mismatch negativity in patients with chronic psychosis. Neuropsychopharmacology 41: 419-430.

Taipale H, Mehtälä J, Tanskanen A, et al. (2018a) Comparative effectiveness of antipsychotic drugs for rehospitalization in schizophrenia-a nationwide study with 20-year follow-up. Schizophr Bull 44: 1381-1387.

Taipale H, Mittendorfer-Rutz E, Alexanderson K, et al. (2018b) Antipsychotics and mortality in a nationwide cohort of 29,823 patients with schizophrenia. Schizophr Res 197: 274-280. 
Tait L, Birchwood M and Trower P (2004) Adapting to the challenge of psychosis: Personal resilience and the use of sealing-over (avoidant) coping strategies. Br J Psychiatry 185: 410-415.

Takeuchi H, Kantor N, Sanches M, et al. (2017) One-year symptom trajectories in patients with stable schizophrenia maintained on antipsychotics versus placebo: Meta-analysis. Br J Psychiatry 211: 137-143.

Takeuchi H, Suzuki T, Uchida H, et al. (2008) Randomized, open-label comparison of 2 switching strategies to aripiprazole treatment in patients with schizophrenia: Add-on, wait, and tapering of previous antipsychotics versus add-on and simultaneous tapering. J Clin Psychopharmacol 28: 540-543.

Takiguchi K, Uezato A, Itasaka M, et al. (2017) Association of schizophrenia onset age and white matter integrity with treatment effect of D-cycloserine: A randomized placebo-controlled double-blind crossover study. BMC Psychiatry 17: 249.

Tanríverdi N and Yazící KM (1996) Immediate recurrence of psychotic symptoms after discontinuation of clozapine: A case report. Biol Psychiatry 39: 814-815.

Tanskanen A, Tiihonen J and Taipale H (2018) Mortality in schizophrenia: 30-year nationwide follow-up study. Acta Psychiatr Scand 138: 492-499.

Tarricone I, Gozzi BF, Serretti A, et al. (2010) Weight gain in antipsychotic-naïve patients: A review and meta-analysis. Psychol Med 40: 187-200.

Tarrier N, Haddock G, Lewis S, et al. (2006) Suicide behaviour over 18 months in recent onset schizophrenic patients: The effects of CBT. Schizophr Res 83: 15-27.

Tarrier N, Lewis S, Haddock G, et al. (2004) Cognitive-behavioural therapy in first-episode and early schizophrenia. 18-month follow-up of a randomised controlled trial. Br J Psychiatry 184: 231-239.

Taylor CL, Stewart R, Ogden J, et al. (2015) The characteristics and health needs of pregnant women with schizophrenia compared with bipolar disorder and affective psychoses. BMC Psychiatry 15: 88 .

Taylor D, Barnes TRE and Young AH (2018) (eds.) The Maudsley Prescribing Guidelines in Psychiatry. 13th ed. Chichester: Wiley Blackwell.

Taylor DM, Smith L, Gee SH, et al. (2012) Augmentation of clozapine with a second antipsychotic - a meta-analysis. Acta Psychiatr Scand 125: $15-24$.

Taylor M and Ng K (2013) Should long-acting (depot) antipsychotics be used in early schizophrenia? A systematic review. Aust N Z J Psychiatry 47: 624-630.

Taylor PJ and Estroff SE (2003) Schizophrenia and violence. In: Hirsch SR and Weinberger WR (eds) Schizophrenia. 2nd edn. Oxford: Blackwell Publishing, pp. 591-612.

The National Confidential Inquiry into Suicide and Safety in Mental Health. (2018) Annual Report: England, Northern Ireland, Scotland, Wales. Manchester: University of Manchester.

Thiels C (1987) Pharmacotherapy of psychiatric disorder in pregnancy and during breastfeeding: A review. Pharmacopsychiatry 20: 133-146.

Thomas KH, Martin RM, Knipe DW, et al. (2015) Risk of neuropsychiatric adverse events associated with varenicline: Systematic review and meta-analysis. BMJ 350: h1 109 .

Thompson A, Winsper C, Marwaha S, et al. (2018) Maintenance antipsychotic treatment versus discontinuation strategies following remission from first episode psychosis: Systematic review. BJPsych Open 4: $215-225$.

Thompson JV, Clark JM, Legge SE, et al. (2016) Antipsychotic polypharmacy and augmentation strategies prior to clozapine initiation: A historical cohort study of 310 adults with treatment-resistant schizophrenic disorders. J Psychopharmacol 30: 436-443.

Thompson K, Kulkarni J and Sergejew AA (2000) Reliability and validity of a new Medication Adherence Rating Scale (MARS) for the psychoses. Schizophr Res 42: 241-247.

Thompson KN, McGorry PD and Harrigan SM (2003) Recovery style and outcome in first-episode psychosis. Schizophr Res 62: 31-36.
Tiihonen J, Haukka J, Taylor M, et al. (2011) A nationwide cohort study of oral and depot antipsychotics after first hospitalization for schizophrenia. Am J Psychiatry 168: 603-609.

Tiihonen J, Lönnqvist J, Wahlbeck K, et al. (2009) 11-year follow-up of mortality in patients with schizophrenia: A population-based cohort study (FIN11 study). Lancet 374: 620-627.

Tiihonen J, Mittendorfer-Rutz E, Majak M, et al. (2017) Real-world effectiveness of antipsychotic treatments in a nationwide cohort of 29823 patients with schizophrenia. JAMA Psychiatry 74: 686-693.

Tiihonen J, Mittendorfer-Rutz E, Torniainen M, et al. (2016) Mortality and cumulative exposure to antipsychotics, antidepressants, and benzodiazepines in patients with schizophrenia: An observational follow-up study. Am J Psychiatry 173: 600-606.

Tiihonen J, Suokas JT, Suvisaari J M, et al. (2012) Polypharmacy with antipsychotics, antidepressants, or benzodiazepines and mortality in schizophrenia. Arch GenPsychiatry 69: 476-483.

Tiihonen J, Taipale H, Mehtälä J, et al. (2019) Association of antipsychotic polypharmacy vs monotherapy with psychiatric rehospitalization among adults with schizophrenia. JAMA Psychiatry 76: 499-507.

Tiihonen J, Tanskanen A and Taipale H (2018) 20-year nationwide follow-up study on discontinuation of antipsychotic treatment in firstepisode schizophrenia. Am J Psychiatry 175: 765-773.

Tiihonen J, Wahlbeck K, Lonnqvist J, et al. (2006) Effectiveness of antipsychotic treatments in a nationwide cohort of patients in community care after first hospitalisation due to schizophrenia and schizoaffective disorder: Observational follow-up study. BMJ 333: 224-229.

Tinazzi M, Morgante F, Matinella A, et al. (2014) Imaging of the dopamine transporter predicts pattern of disease progression and response to levodopa in patients with schizophrenia and parkinsonism: A 2-year follow-up multicentre study. Schizophr Res 152: 344-349.

Titus-Lay EN, Ansara ED, Isaacs AN, et al. (2018) Evaluation of adherence and persistence with oral versus long-acting injectable antipsychotics in patients with early psychosis. Ment Health Clin 8: 56-62.

Toftdahl NG, Nordentoft M and Hjorthøj C (2016) Prevalence of substance use disorders in psychiatric patients: A nationwide Danish population-based study. Soc Psychiatry Psychiatr Epidemiol 51: 129-140.

Tollefson GD, Beasley CM Jr, Tran PV, et al. (1997) Olanzapine versus haloperidol in the treatment of schizophrenia and schizoaffective and schizophreniform disorders: Results of an international collaborative trial. Am J Psychiatry 154: 457-465.

Tollefson GD, Birkett MA, Kiesler GM, et al. (2001) Double-blind comparison of olanzapine versus clozapine in schizophrenic patients clinically eligible for treatment with clozapine. Biol Psychiatry 49: 52-63.

Tomson T and Battino D (2012) Teratogenic effects of antiepileptic drugs. Lancet Neurol 11: 803-813.

Tormoehlen LM and Rusyniak DE (2018) Neuroleptic malignant syndrome and serotonin syndrome. In: Romanovsky AA (ed) Handbook of Clinical Neurology, Vol 157 (3rd Series): Thermoregulation: From Basic Neuroscience to Clinical Neurology: Part II. Amsterdam: Elsevier, pp. 663-676.

Torniainen M, Mittendorfer-Rutz E, Tanskanen A, et al. (2015) Antipsychotic treatment and mortality in schizophrenia. Schizophr Bull 41: 656-663.

Toxicology Data Network (2019) Drugs and Lactation Dabase - LactMed. Available at: https://toxnet.nlm.nih.gov/newtoxnet/lactmed.htm.

Tregellas JR, Tanabe J, Rojas DC, et al. (2011) Effects of an alpha 7-nicotinic agonist on default network activity in schizophrenia. Biol Psychiatry 69: 7-11.

Tsang HW, Fung KM and Chung RC (2010) Self-stigma and stages of change as predictors of treatment adherence of individuals with schizophrenia. Psychiatry Res 180: 10-15.

Tse L, Barr AM, Scarapicchia V, et al. (2015) Neuroleptic malignant syndrome: Review from a clinically oriented perspective. Curr Neuropharmacol 13: 395-406. 
Tseng PT, Chen YW, Chung W, et al. (2016) Significant effect of valproate augmentation therapy in patients with schizophrenia: A metaanalysis study. Medicine (Baltimore) 95: e2475.

Tundo A, de Filippis R and Proietti L (2015) Pharmacologic approaches to treatment resistant depression: Evidences and personal experience. World J Psychiatry 5: 330.

Uchida H, Suzuki T, Takeuchi H, et al. (2011a) Low dose vs standard dose of antipsychotics for relapse prevention in schizophrenia: Metaanalysis. Schizophr Bull 37: 788-799.

Uchida H, Takeuchi H, Graff-Guerrero A, et al. (2011b) Predicting dopamine D2 receptor occupancy from plasma levels of antipsychotic drugs: A systematic review and pooled analysis. J Clin Psychopharmacol 31: 318-325.

Üçok A, Serbest S and Kandemir PE. (2011) Remission after first-episode schizophrenia: Results of a long-term follow-up. Psychiatry Res 189: 33-37.

Uguz F (2016) Second-generation antipsychotics during the lactation period: A comparative systematic review on infant safety. J Clin Psychopharmacol 36: 244-252.

Umbricht D, Keefe RS, Murray S, et al. (2014) A randomized, placebocontrolled study investigating the nicotinic alpha7 agonist, RG3487, for cognitive deficits in schizophrenia. Neuropsychopharmacology 39: $1568-1577$.

Upthegrove R, Birchwood M, Ross K, et al. (2010) The evolution of depression and suicidality in first episode psychosis. Acta Psychiat Scand 122: 211-218.

Upthegrove R, Marwaha S and Birchwood M (2017) Depression and schizophrenia: Cause, consequence, or trans-diagnostic issue? Schizophr Bull 43: 240-244.

Upthegrove R, Ross K, Brunet K, et al. (2014) Depression in first episode psychosis: The role of subordination and shame. Psychiatry Res 217: 177-184.

Valenstein M, Ganoczy D, McCarthy JF, et al. (2006) Antipsychotic adherence over time among patients receiving treatment for schizophrenia: A retrospective review. J Clin Psychiatry 67: 1542-1550.

Valmaggia L, Byrne M, Day F, et al. (2015) Duration of untreated psychosis and need for admission in patients who engage with mental health services in the prodromal phase. Br J Psychiatry 207: 130-134.

Valmaggia LR, McCrone P, Knapp M, et al. (2009) Economic impact of early intervention in people at high risk of psychosis. Psychol Med 39: $1617-1626$

van der Gaag M, Smit F, Bechdolf A, et al. (2013) Preventing a first episode of psychosis: Meta-analysis of randomized controlled prevention trials of 12 month and longer-term follow-ups. Schizophr Res 149: 56-62.

Van Harten PN, Hoek HW, Matroos GE, et al. (1998) Intermittent neuroleptic treatment and risk of tardive dyskinesia: Curacao Extrapyramidal Syndromes Study III. Am J Psychiatry 155: 565-567.

van Nimwegen LJ, de Haan L, van Beveren NJ, et al. (2008) Effect of olanzapine and risperidone on subjective well-being and craving for cannabis in patients with schizophrenia or related disorders: A double blind randomized controlled trial. Can J Psychiatry 53: 400-405.

van Noord C, Sturkenboom MC, Straus SM, et al. (2011) Non-cardiovascular drugs that inhibit hERG-encoded potassium channels and risk of sudden cardiac death. Heart 97: 215-220.

Vancampfort D, Wampers M, Mitchell AJ, et al. (2013) A meta-analysis of cardio-metabolic abnormalities in drug naive, first-episode and multi-episode patients with schizophrenia versus general population controls. World Psychiatry 12: 240-250.

Vandael E, Vandenberk B, Vandenberghe J, et al. (2017) Risk factors for QTc-prolongation: Systematic review of the evidence. Int J Clin Pharm 39: 16-25.

Vandenberghe F, Gholam-Rezaee M and Saigí-Morgui N (2015) Importance of early weight changes to predict long-term weight gain during psychotropic drug treatment. J Clin Psychiatry 76: e1417-e1423.
Vayısoğlu S, Anıl Yağcioğlu AE, Yağcioğlu S, et al. (2013) Lamotrigine augmentation in patients with schizophrenia who show partial response to clozapine treatment. Schizophr Res 143: 207-214.

Veerman SR, Schulte PF, Begemann MJ, et al. (2014) Clozapine augmented with glutamate modulators in refractory schizophrenia: A review and metaanalysis. Pharmacopsychiatry 47: 185-194.

Velamoor VR, Norman RM, Caroff SN, et al. (1994) Progression of symptoms in neuroleptic malignant syndrome. J Nerv Ment Dis 182: 168-173.

Velligan DI, Lam F, Ereshefsky L, et al. (2003) Psychopharmacology: Perspectives on medication adherence and atypical antipsychotic medications. Psychiatr Serv 54: 665-667.

Velligan DI, Weiden PJ, Sajatovic M, et al. (2009) The expert consensus guideline series: Adherence problems in patients with serious and persistent mental illness. J Clin Psychiatry 70(Suppl 4): 1-46.

Vergouwen AC, Bakker A and Koerselman F (2003) Adherence to medication for chronic psychiatric diseases; determining the optimum frequency and form of administration. Am J Drug Deliv 1: 267-273.

Vermeulen J, van Rooijen G, Doedens P, et al. (2017) Antipsychotic medication and long-term mortality risk in patients with schizophrenia; a systematic review and meta-analysis. Psychol Med 47: 2217-2228.

Veroniki AA, Rios P, Cogo E, et al. (2017) Comparative safety of antiepileptic drugs for neurological development in children exposed during pregnancy and breast feeding: A systematic review and network meta-analysis. BMJ Open 7: $\mathrm{e} 017248$.

Vervloet M, Linn AJ, van Weert JC, et al. (2012) The effectiveness of interventions using electronic reminders to improve adherence to chronic medication: A systematic review of the literature. $\mathrm{J} \mathrm{Am} \mathrm{Med}$ Inform Assoc 19: 696-704.

Vieweg WV, Hasnain M, Howland RH, et al. (2012) Citalopram, QTc interval prolongation, and torsade de pointes. How should we apply the recent FDA ruling? Am J Med 125: 859-868.

Victoroff J, Coburn K, Reeve A, et al. (2014) Pharmacological management of persistent hostility and aggression in persons with schizophrenia spectrum disorders: A systematic review. J Neuropsychiatry Clin Neurosci 26: 283-312.

Vigod SN, Gomes T, Wilton AS, et al. (2015) Antipsychotic drug use in pregnancy: High dimensional, propensity matched, population based cohort study. BMJ 550: h2298.

Vigod SN, Kurdyak PA, Dennis CL, et al. (2014) Maternal and newborn outcomes among women with schizophrenia. BJOG 121: $566-574$.

Viguera AC, Baldessarini RJ, Hegarty JD, et al. (1997) Clinical risk following abrupt and gradual withdrawal of maintenance neuroleptic treatment. Arch Gen Psychiatry 54: 49-55.

Villalta-Gil V, Vilaplana M, Ochoa S, et al. (2006) Neurocognitive performance and negative symptoms: Are they equal in explaining disability in schizophrenia outpatients? Schizophr Res 87: 246-253.

Volavka J and Citrome L (1999) Atypical antipsychotics in the treatment of the persistently aggressive psychotic patient: Methodological concerns. Schizophr Re 35(Suppl): S23-S33.

Volavka J and Citrome L (2008) Heterogeneity of violence in schizophrenia and implications for long-term treatment. Int $J$ Clin Pract 62: $1237-1245$.

Volavka J and Citrome L (2011) Pathways to aggression in schizophrenia affect results of treatment. Schizophr Bull 37: 921-929.

Volavka J, Czobor P, Citrome L, et al. (2014) Effectiveness of antipsychotic drugs against hostility in patients with schizophrenia in the Clinical Antipsychotic Trials of Intervention Effectiveness (CATIE) study. CNS Spectr 19: 374-381.

Voss EA, Ryan PB, Stang PE, et al. (2015) Switching from risperidone long-acting injectable to paliperidone long-acting injectable or oral antipsychotics: Analysis of a Medicaid claims database. Int Clin Psychopharmacol 30: 151-157. 
Waddell L and Taylor M (2009) Attitudes of patients and mental health staff to antipsychotic long-acting injections: Systematic review. $\mathrm{Br} J$ Psychiatry 195: s43-s50.

Walling D, Marder SR, Kane J, et al. (2016) Phase 2 trial of an alpha-7 nicotinic receptor agonist (TC-5619) in negative and cognitive symptoms of schizophrenia. Schizophr Bull 42: 335-343.

Wang J, Omori IM, Fenton M, et al. (2010) Sulpiride augmentation for schizophrenia. Cochrane Database Syst Rev 1: CD008125.

Wang Y, Xia J, Helfer B, et al. (2016) Valproate for schizophrenia. Cochrane Database Syst Rev 11: CD004028.

Ward A, Ishak K, Proskorovsky I, et al. (2006) Compliance with refilling prescriptions for atypical antipsychotic agents and its association with the risks for hospitalization, suicide, and death in patients with schizophrenia in Quebec and Saskatchewan: A retrospective database study. Clin Ther 11: 1912-1921.

Ware MR, Feller DB and Hall KL (2018) Neuroleptic malignant syndrome: Diagnosis and management. Prim Care Companion CNS Disord 20: pii 17r02185.

Weickert TW and Weickert CS (2017) Raloxifene improves cognition in schizophrenia: Spurious result or valid effect? Front Psychiatry 8: 202.

Weickert TW, Weinberg D, Lenroot R, et al. 2015. Adjunctive raloxifene treatment improves attention and memory in men and women with schizophrenia. Mol Psychiatry 20: 685-694.

Weiden PJ (2006) Switching in the era of atypical antipsychotics. An updated review. Postgrad Med Spec No: 27-44.

Weiden PJ, Kozma C, Grogg A, et al. (2004) Partial compliance and risk of rehospitalization among California Medicaid patients with schizophrenia. Psychiatr Serv 55: 886-891.

Weiden PJ, Preskorn SH, Fahnestock PA, et al. (2007) translating the psychopharmacology of antipsychotics to individualized treatment for severe mental illness: A roadmap. J Clin Psychiatry 68(Suppl 7): $1-48$.

Weiden PJ and Zygmunt A (1997) The road back: Working with the severely mentally ill. Medication noncompliance in schizophrenia: I. assessment. J Prac Psych Behav Health 3: 106-110.

Weinmann S, Read J and Aderhold V (2009) Influence of antipsychotics on mortality in schizophrenia: Systematic review. Schizophr Res 113: $1-11$.

Weintraub D, Chahine LM, Hawkins KA, et al. (2017) Cognition and the course of prodromal Parkinson's disease. Mov Disord 32: 1640-1645.

Weiser M, Heresco-Levy U, Davidson M, et al. (2012) A multicenter, add-on randomized controlled trial of low-dose D-serine for negative and cognitive symptoms of schizophrenia. J Clin Psychiatry 73: e728-e734.

Weiser M, Levi L, Burshtein S, et al. (2017) Raloxifene plus antipsychotics versus placebo plus antipsychotics in severely ill decompensated postmenopausal women with schizophrenia or schizoaffective disorder: A randomized controlled trial. J Clin Psychiatry 78: e758-e765.

Weiser M, Levi L, Burshtein S, et al. (2019a) The effect of minocycline on symptoms in schizophrenia: Results from a randomized controlled trial. Schizophr Res 206: 325-332.

Weiser M, Levi L, Zamora D, et al. (2019b) Effect of adjunctive estradiol on schizophrenia among women of childbearing age: A randomized clinical trial. JAMA Psychiatry. Epub ahead of print 31 July 2019. doi: $10.1001 /$ jamapsychiatry.2019.1842

Wells A, Sommi R and Crismon M (1988) Neuroleptic rechallenge after neuroleptic malignant syndrome: Case report and literature review. Drug Intell Clin Pharm 22: 475-480.

West JC, Marcus SC, Wilk J, et al. (2008) Use of depot antipsychotic medications for medication nonadherence in schizophrenia. Schizophr Bull 34: 995-1001.

Westin AA, Brekke M, Molden E, et al. (2018) Treatment with antipsychotics in pregnancy: Changes in drug disposition. Clin Pharmacol Ther 103: 477-484.
Whitehead C, Moss S, Cardno A, et al. (2002) Antidepressants for people with both schizophrenia and depression. Cochrane Database Syst Rev CD002305-CD002305.

Whitehead C, Moss S, Cardno A, et al. (2003) Antidepressants for the treatment of depression in people with schizophrenia: A systematic review. Psychol Med 33: 589-599.

Wilder CM, Elbogen EB, Moser LL, et al. (2010) Medication preferences and adherence among individuals with severe mental illness and psychiatric advance directives. Psychiatr Serv 61: 380-385.

Wilson RD, Audibert F, Brock JA, et al. (2015) Pre-conception folic acid and multivitamin supplementation for the primary and secondary prevention of neural tube defects and other folic acid-sensitive congenital anomalies. J Obstet Gynaecol Can 37: 534-552.

Wilson WH and Claussen AM (1995) 18-month outcome of clozapine treatment for 100 patients in a state psychiatric hospital. Psychiatr Serv 46: 386-389.

Wimberley T, MacCabe JH, Laursen TM, et al. (2017) Mortality and self-harm in association with clozapine in treatment-resistant schizophrenia. Am J Psychiatry 174: 990-998.

Windfuhr K, Turnbull P, While D, et al. (2011) The incidence and associated risk factors for sudden unexplained death in psychiatric inpatients in England and Wales. J Psychopharmacol 25: 1533-1542.

Winsper C, Singh SP, Marwaha S, et al. (2013) Pathways to violent behavior during first-episode psychosis: A report from the UK National EDEN Study. JAMA Psychiatry 70: 1287-1293.

Winterer G, Gallinat J, Brinkmeyer J, et al. (2013) Allosteric alpha-7 nicotinic receptor modulation and P50 sensory gating in schizophrenia: A proof-of-mechanism study. Neuropharmacology 64: 197-204.

Wobrock T and Soyka M (2009) Pharmacotherapy of patients with schizophrenia and substance abuse. Expert Opin Pharmacother 10: 353-367.

Woerner MG, Kane JM, Lieberman JA, et al. (1991) The prevalence of tardive dyskinesia. J Clin Psychopharmacol 11: 34-42.

Wong-Anuchit C, Chantamit-O-Pas C, Schneider JK, et al. (2019) Motivational interviewing-based compliance/adherence therapy interventions to improve psychiatric symptoms of people with severe mental illness: Meta-analysis. J Am Psychiatr Nurses Assoc 25: 122-133.

Woods S, Saksa J, Compton M, et al. (2017) Effects of ziprasidone versus placebo in patients at clinical high risk for psychosis. Schizophr Bull 43: S58.

Woods SW, Brier A, Zipursky RB, et al. (2003) Randomized trial of olanzapine versus placebo in the symptomatic acute treatment of the schizophrenic prodrome. Biol Psychiatry 54: 453-464.

Woods SW, Tully E, Walsh B, et al. (2007) Aripiprazole in the treatment of the psychosis prodrome: An open-label pilot study. Br J Psychiatry 191: s96-s101.

World Health Organization (1992) The ICD-10 International Classification of Diseases and Related Health Problems (Tenth Revision). Geneva: WHO.

Woodward ND, Purdon SE, Meltzer HY, et al. (2005) A meta-analysis of neuropsychological change to clozapine, olanzapine, quetiapine, and risperidone in schizophrenia. Int J Neuropsychopharmacol 8: 457-472.

Wu CS, Tsai YT and Tsai HJ (2015) Antipsychotic drugs and the risk of ventricular arrhythmia and/or sudden cardiac death: A nation-wide case-crossover study. J Am Heart Assoc 4: pii: e001568.

Wu Q, Gilbody S, Peckham E, et al. (2016) Varenicline for smoking cessation and reduction in people with severe mental illnesses: Systematic review and meta-analysis. Addiction 111: 1554-1567.

Wunderink L, Nieboer RM, Wiersma D, et al. (2013) Recovery in remitted first-episode psychosis at 7 years of follow-up of an early dose reduction/discontinuation or maintenance treatment strategy: Longterm follow-up of a 2-year randomized clinical trial. JAMA Psychiatry 70: 913-920.

Wunderink L, Nienhuis FJ, Sytema S, et al. (2007) Guided discontinuation versus maintenance treatment in remitted first-episode psychosis: Relapse rates and functional outcome. J Clin Psychiatry 68: 654-661. 
Wyatt RJ (1997) Research in schizophrenia and the discontinuation of antipsychotic medications. Schizophr Bull 23: 3-9.

Xiang YQ, Zheng W, Wang SB, et al. (2017) Adjunctive minocycline for schizophrenia: A meta-analysis of randomized controlled trials. Eur Neuropsychopharmacol 27: 8-18.

Yang J, Ko YH, Paik JW, et al. (2012) Symptom severity and attitudes toward medication: Impacts on adherence in outpatients with schizophrenia. Schizophr Res 134: 226-231.

Yang L, Link B, Ben-David S, et al. (2015) Stigma related to labels and symptoms in individuals at clinical high-risk for psychosis. Schizophr Res 168: 9-15.

Yap YG and Camm AJ (2003) Drug induced QT prolongation and torsades de pointes. Heart 89: 1363-1372.

Yin J, Barr AM, Ramos-Miguel A, et al. (2017) Antipsychotic induced dopamine supersensitivity psychosis: A comprehensive review. Curr Neuropharmacol 15: 174-183.

Yoshimura B, Yada Y, So R, et al. (2017) The critical treatment window of clozapine in treatment-resistant schizophrenia: Secondary analysis of an observational study. Psychiatry Res 250: 65-70.

Yung AR, Buckby JA, Cotton SM, et al. (2006) Psychotic-like experiences in nonpsychotic help seekers: Associations with distress, depression, and disability. Schizophr Bull 32: 352-359.

Yung AR, McGorry PD, McFarlane CA, et al. (1996) Monitoring and care of young people at incipient risk of psychosis. Schizophr Bull 22: 283-303.

Yung AR, Phillips LJ, Nelson B, et al. (2011) Randomized controlled trial of interventions for young people at Ultra High Risk of psychosis: 6 month analysis. J Clin Psychiatry 72: 430-440.

Yung AR, Yuen HP, Berger G, et al. (2007) Declining transition rate in Ultra High Risk (prodromal) services: Dilution or reduction of risk? Schizophr Bull 33: 673-681.

Yusufi B, Mukherjee S, Flanagan R, et al. (2007) Prevalence and nature of side effects during clozapine maintenance treatment and the relationship with clozapine dose and plasma concentration. Int Clin Psychopharmacol 22: 238-243.

Ziegenbein M and Calliess IT (2006) Clozapine and ziprasidone: A useful combination in patients with treatment-resistant schizophrenia. $J$ Neuropsychiatry Clin Neurosci 18: 246-247.

Ziegenbein M1, Kropp S and Kuenzel HE (2005) Combination of clozapine and ziprasidone in treatment-resistant schizophrenia: An open clinical study. Clin Neuropharmacol 28: 220-224.

Zhang C, Chen MJ, Wu GJ, et al. (2016) Effectiveness of antipsychotic drugs for 24-month maintenance treatment in first-episode schizophrenia: Evidence from a community-based "real-world" study. $J$ Clin Psychiatry 77: e1460-e1466.
Zhang JP, Gallego JA, Robinson DG, et al. (2013) Efficacy and safety of individual second-generation vs. first-generation antipsychotics in first-episode psychosis: A systematic review and meta-analysis. Int $J$ Neuropsychopharmacol 16: 1205-1218.

Zhang L and Zhao J (2014) Profile of minocycline and its potential in the treatment of schizophrenia. Neuropsychiatr Dis Treat 10: 11031111.

Zhang XY, Liu L, Liu S, et al. (2012) Short-term tropisetron treatment and cognitive and P50 auditory gating deficits in schizophrenia. Am J Psychiatry 169: 974-981.

Zheng W, Cai D-B, Yang X-H, et al. (2017a) Adjunctive celecoxib for schizophrenia: A meta-analysis of randomized, double-blind, placebo-controlled trials. J Psychiatr Res 92: 139-146.

Zheng W, Li XH, Yang X-H, et al. (2018) Adjunctive memantine for schizophrenia: A meta-analysis of randomized, double-blind, placebo-controlled trials. Psychol Med 48: 72-81.

Zheng W, Xiang YT, Yang XH, et al. (2017b) Clozapine augmentation with antiepileptic drugs for treatment-resistant schizophrenia: A meta-analysis of randomized controlled trials. J Clin Psychiatry 78: e498-e505.

Zheng W, Zhu XM, Zhang QE, et al. (2019) Adjunctive minocycline for major mental disorders: A systematic review. J Psychopharmacol 33: 1215-1226.

Zhou X, Keitner GI, Qin B, et al. (2015) Atypical antipsychotic augmentation for treatment-resistant depression: A systematic review and network meta-analysis. Int $J$ Neuropsychopharmacol 18 : pyv060.

Zhou Y, Li G, Li D, et al. (2018) Dose reduction of risperidone and olanzapine can improve cognitive function and negative symptoms in stable schizophrenic patients: A single-blinded, 52-week, randomized controlled study. J Psychopharmacol 32: 524-532.

Zhu B, Ascher-Svanum H, Shi L, et al. (2008) Time to discontinuation of depot and oral first-generation antipsychotics in the usual care of schizophrenia. Psychiatr Serv 59: 315-317.

Zhu Y, Li C, Huhn M, Rothe P, et al. (2017) How well do patients with a first episode of schizophrenia respond to antipsychotics: A systematic review and meta-analysis. Eur Neuropsychopharmacol 27: 835-844.

Zimbroff D, Warrington L, Loebel A, et al. (2007) Comparison of ziprasidone and aripiprazole in acutely ill patients with schizophrenia or schizoaffective disorder: A randomized, double-blind, 4-week study. Int Clin Psychopharmacol 22: 363-370.

Zipursky RB, Menezes NM and Streiner DL (2014) Risk of symptom recurrence with medication discontinuation in first-episode psychosis: A systematic review. Schizophr Res 152: 408-414. 